\title{
WestVirginiaUniversity
}

THE RESEARCH REPOSITORY @ WVU

Graduate Theses, Dissertations, and Problem Reports

2006

\section{Soluble metals of residual oil fly ash alter pulmonary host defense in rats}

Jenny Renee Roberts

West Virginia University

Follow this and additional works at: https://researchrepository.wvu.edu/etd

\section{Recommended Citation}

Roberts, Jenny Renee, "Soluble metals of residual oil fly ash alter pulmonary host defense in rats" (2006). Graduate Theses, Dissertations, and Problem Reports. 2404.

https://researchrepository.wvu.edu/etd/2404

This Dissertation is protected by copyright and/or related rights. It has been brought to you by the The Research Repository @ WVU with permission from the rights-holder(s). You are free to use this Dissertation in any way that is permitted by the copyright and related rights legislation that applies to your use. For other uses you must obtain permission from the rights-holder(s) directly, unless additional rights are indicated by a Creative Commons license in the record and/ or on the work itself. This Dissertation has been accepted for inclusion in WVU Graduate Theses, Dissertations, and Problem Reports collection by an authorized administrator of The Research Repository @ WVU.

For more information, please contact researchrepository@mail.wvu.edu. 


\title{
SOLUBLE METALS OF RESIDUAL OIL FLY ASH ALTER PULMONARY HOST DEFENSE IN RATS
}

\author{
Jenny Renee Roberts \\ Dissertation submitted to the School of Medicine at \\ West Virginia University \\ in partial fulfillment of the requirements for the degree of \\ Doctor of Philosophy \\ in \\ Physiology and Pharmacology \\ Vincent Castranova, Ph. D., Chair \\ Richard Dey, Ph. D. \\ Ronald Millecchia, Ph. D. \\ Dale Porter, Ph. D. \\ Mark Reasor, Ph. D. \\ Department of Physiology and Pharmacology \\ Morgantown, West Virginia \\ Copyright 2006, Jenny R. Roberts
}

Keywords: Residual Oil Fly Ash; Listeria monocytogenes; Metals; Pulmonary Host Defense; Alveolar Macrophage; Cytokines; Oxidants; T Cells 


\section{ABSTRACT \\ SOLUBLE METALS OF RESIDUAL OIL FLY ASH ALTER PULMONARY HOST DEFENSE IN RATS}

\section{Jenny Renee Roberts}

Incidences of high levels of air pollution have been correlated with increased morbidity and mortality in susceptible populations. Inhalation of the combustion-derived pollutant, residual oil fly ash (ROFA), has been shown to impair lung defense mechanisms in laboratory animals and susceptible populations. The hypothesis of these studies was that the soluble metals in ROFA would increase susceptibility to pulmonary infection in rats, and that the primary metal responsible for the increase is soluble Ni. To investigate the hypothesis, three specific aims were established: 1) determine if soluble metals in ROFA cause a decrease in bacterial clearance from the lungs of rats, 2) determine the potential mechanisms by which the soluble metals suppress the innate and adaptive pulmonary immune response to infection, and 3) establish what metal or metal combination would account for the alterations in pulmonary host defense.

To investigate this, an in vivo infectivity model was employed where adult rats were intratracheally instilled with ROFA (R-Total), soluble (R-Soluble) or insoluble ROFA (R-Insoluble), or the major individual soluble metals or metal combinations in ROFA on day 0. Control groups included the vehicle control (phosphate-buffered saline) and the soluble ROFA sample after metals had been extracted from the solution by chelation (R-Chelex). On day 3, rats were intratracheally inoculated with Listeria monocytogenes as the model pathogen. Pulmonary clearance, morbidity, cellular profiles and phenotypes, lung injury parameters, and cytokine and oxidant production were monitored on day 3 prior to infection, and for 1 week after infection (days 6, 8, and 10).

The first study revealed that the primary metal constituents of ROFA were iron $(\mathrm{Fe})$, nickel (Ni), vanadium (V), aluminum (Al), and zinc ( $\mathrm{Zn})$. The soluble fraction of ROFA contained Fe, Ni, Al, and $\mathrm{Zn}$, but very little V. R-Soluble was found to significantly decrease bacterial clearance from the lungs of rats to a similar degree as RTotal, whereas R-Insoluble did not alter clearance of bacteria when compared to control. In addition, removal of the metals from R-Soluble abolished the increase in susceptibility to infection. In study 2 , the R-Soluble sample was shown to induce a lung injury and inflammation prior to and post-infection which was comparable to, if not more severe than, R-Total. Post-infection, R-Soluble was found to increase neutrophil, macrophage, and lymphocyte influx into the lungs, and to increase NO and IL-6 production, indicating an exacerbated acute phase response and excess inflammation in this group. In addition, macrophage function appeared to be inhibited, as indicated by the increased bacterial burdens at all time points in the R-Soluble group, and T cell activity was also suppressed, demonstrated as reduced interleukin (IL)-2 and IL-4. These results were replicated in study 3 with the artificial mixture of soluble $\mathrm{Fe}, \mathrm{Ni}, \mathrm{Al}$, and $\mathrm{Zn}$. Exposure to the individual metals indicated that soluble $\mathrm{Ni}$, alone or in a metal combination, was largely responsible for the responses observed in the R-Soluble sample in study 2.

These three studies indicate that the soluble metals in ROFA induced lung injury and inflammation, and altered both innate and adaptive immune responses in rats after infection with L. monocytogenes. The suppression of the innate immune response and inhibition of $\mathrm{T}$ cell function appeared to be highly dependent on soluble Ni. Therefore, the metal composition and the bioavailability of the metals in particulate matter may ultimately be the major determinants of the potential for adverse health effects, such as increased susceptibility to infection. 


\section{DEDICATION}

I would to dedicate this to my husband, Jim, whose love and support throughout was invaluable to me, and to my parents, who instilled in me the value an education. 


\section{ACKNOWLEDGEMENTS}

There a number people I would like to acknowledge and thank for their support and guidance. I would like thank my committee members, past and persent. For this I need to thank Dr. Richard Dey, Dr. Mark Reasor, Dr. Dale Porter, Dr. Ronald Millecchia, and Dr. Ping Lee for all of their input and guidance. I would like to give a special thanks to my advisor and committee chair, Dr. Castranova, for all of his advice, mentoring, support, and provision of an ideal setting for a graduate student to perform research. I also need to acknowledge another mentor of mine, Dr. Rick Rogers. If it weren't for Rick, I probably would never have pursued a career in respiratory research. I need to thank Dr. Clarke and Dr. Schafer for the supplying the agents needed to complete this work.

I would also like to extend my thanks to NIOSH for the financial support for the project through the Antonini Lab. Without the support of my co-workers in the lab, particularly Shi-Houng Young, this work would not be possible. Thanks to my colleagues and friends, Jim Scabilloni, Ashley Murray, and Elena Kisin, for all the fun at the end of the hall.

No man is a failure who has friends. For what I have accomplished in my life, I would like to thank my family and friends. I would like to thank my parents, the Ruhe family, the Roberts family, and to my grandparents, who have since passed on. I would like to extend a special thanks to Amelia Mueller for all of her friendship, her humor, and her support since way back when. I would also like to give a special thanks to Tara McNamara for 26 years of friendship, and many more to come. Thanks to the friends that I made in Boston- Robert Clarke, Kafi Meadows, Emily Sullivan, and Andrea Patterson. If it had not been for my first research job at the Harvard School of Public Health and Jim Antonini, I would not be here today.

In 1999, my life changed forever, and Rogers McAvoy lost a dollar. I would like to thank all the people that make me proud to say that West Virginia is my home. Thanks to Jim's parents, Jim and Diana, for their love and support. Thanks to the friends in Morgantown - Mike and Joni Roh, the Davis brothers, Charles "beeeooop" Randolph, Shey Scheutzner, Alicia and Kirk Bebout, Mike and Nicole Moran, Al Bonner and Julie Wilson, Pam Murray, Lucy Morrison, and the extended family at Gene's Beer Garden. I would like to extend my gratitude and a special thanks to the President's Day Crew past, present, and future.

Finally, I would like to acknowledge the most important person in my life, without whom I would not be where I am today. Thanks to my husband Jim for supporting me and putting up with me through all of this. You make me a better person. 


\section{TABLE OF CONTENTS}

ABSTRACT

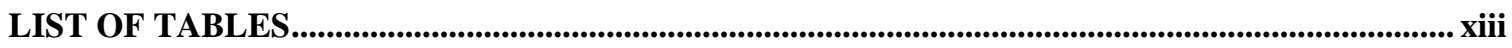

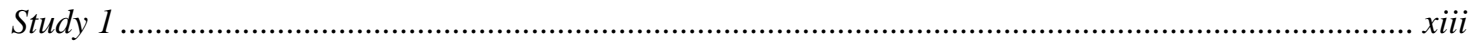

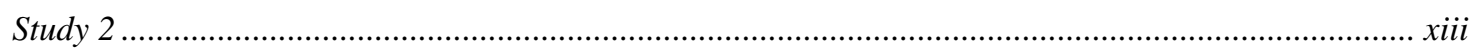

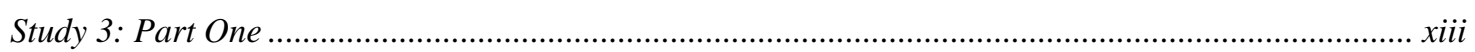

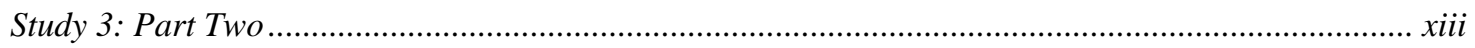

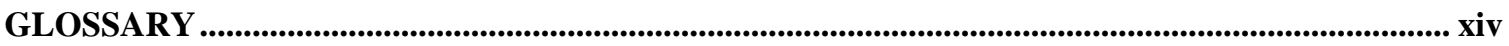

LITERATURE REVIEW .............................................................................................................. 1

Air Pollutant Particulate Matter and Associated Health Effects........................................................... 1

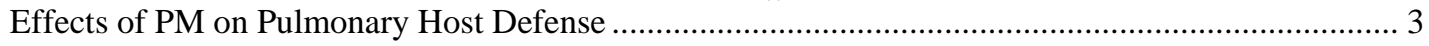

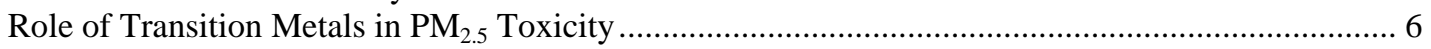

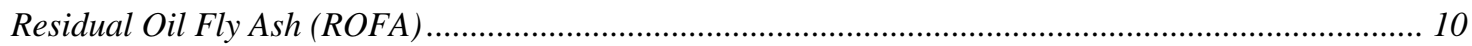

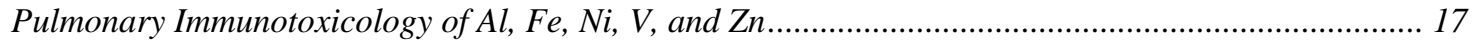

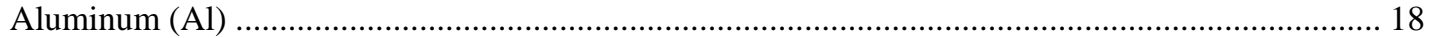

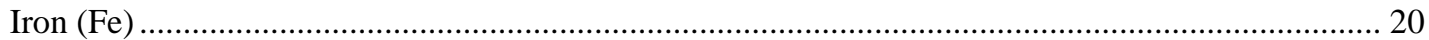

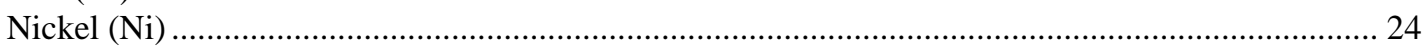

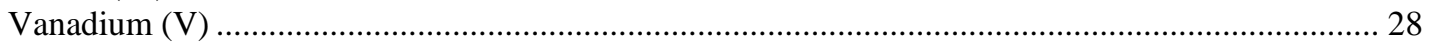

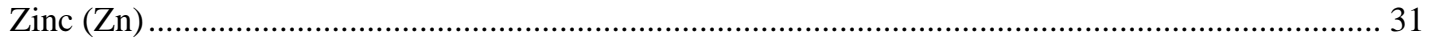

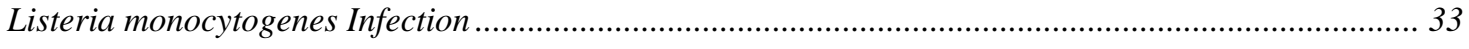

Hypothesis and Specific Aims ............................................................................................... 38

STUDY 1: SOLUBLE METALS ASSOCIATED WITH RESIDUAL OIL FLY ASH INCREASE

MORBIDITY AND LUNG INJURY AFTER BACTERIAL INFECTION IN RATS .......................... 40

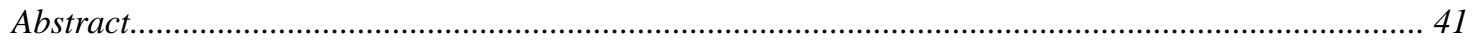

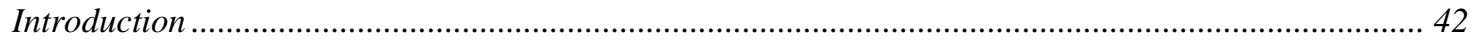

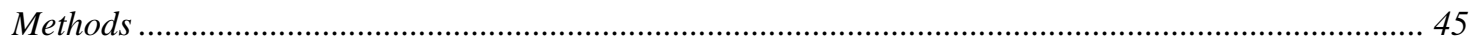

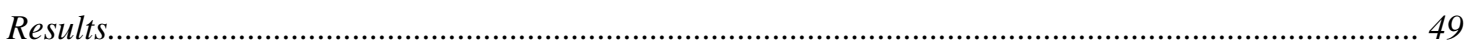

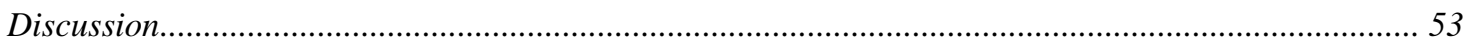




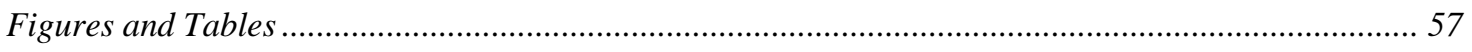

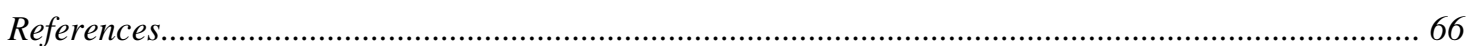

STUDY 2: ALTERATIONS IN INNATE AND ADAPTIVE PULMONARY IMMUNE RESPONSES TO BACTERIAL INFECTION IN THE LUNGS OF RATS EXPOSED TO THE SOLUBLE

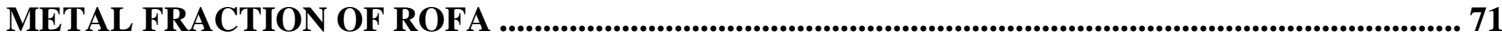

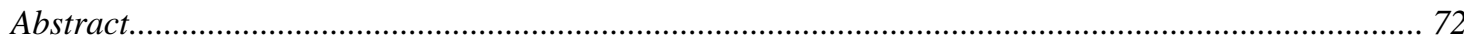

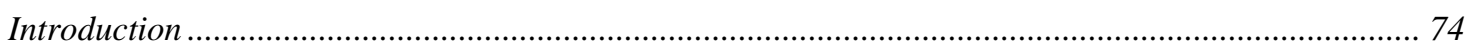

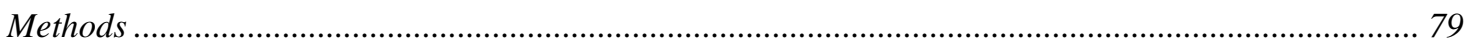

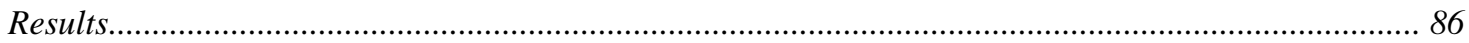

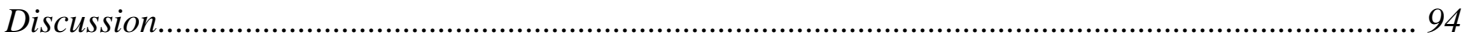

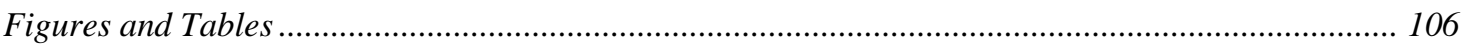

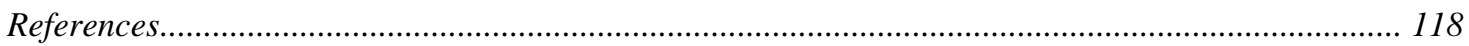

STUDY 3: SOLUBLE NICKEL, A COMPONENT OF RESIDUAL OIL FLY ASH, ALTERS

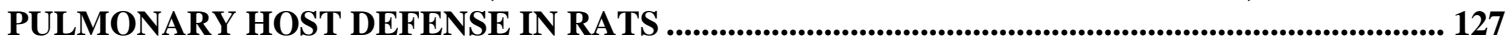

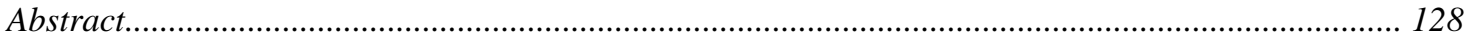

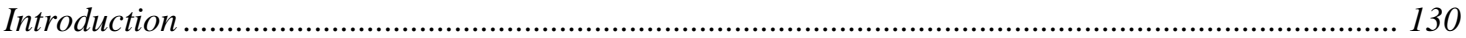

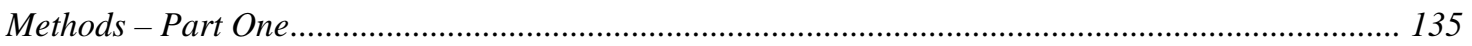

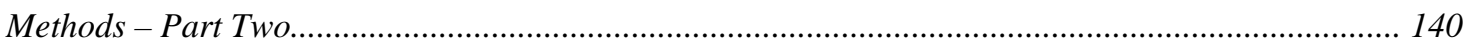

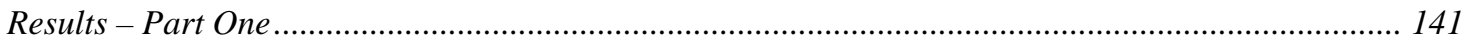

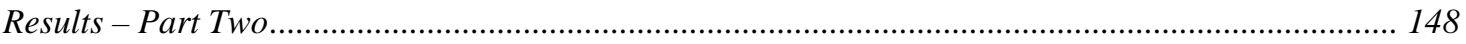

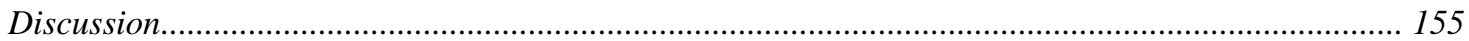

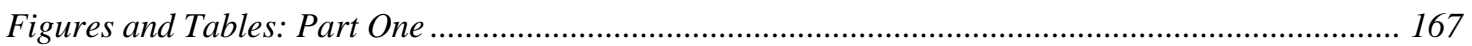

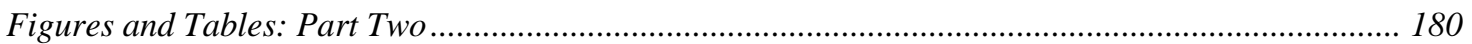

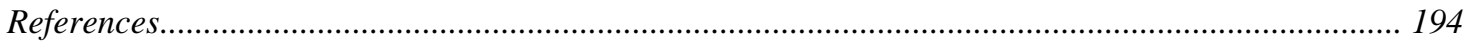

GENERAL DISCUSSION ...................................................................................................................... 203

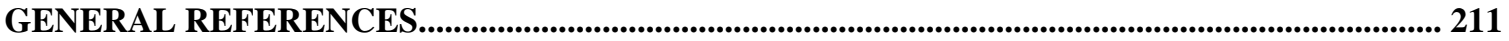

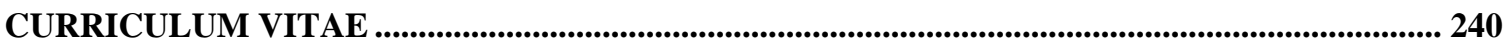




\section{LIST OF FIGURES}

\section{Study 1}

Figure 1.1a. Scanning electron micrographs of ROFA particles representing the size distribution....................................................................57

Figure 1.1b. Scanning electron micrographs of ROFA particles representing the size distribution.............................................................57 Figure $1.2 \mathrm{a}$. \% change in body weight post-infection of rats that were pre-exposed to RTotal, R-Soluble, R-Insoluble, or Saline by intratracheal instillation 3 days prior intratracheal inoculation with $5 \times 10^{5} \mathrm{~L}$. monocytogenes..........................58 Figure 1.2b. \% change in body weight post-infection of rats that were pre-exposed to RTotal, R-Soluble, R-Insoluble, or Saline by intratracheal instillation 3 days prior intratracheal inoculation with $5 \times 10^{4} \mathrm{~L}$. monocytogenes..........................58 Figure 1.3a. Number of bacterial CFUs in the left lung of rats that were pre-exposed to RTotal, R-Soluble, R-Insoluble, or Saline by intratracheal instillation 3 days prior to intratracheal inoculation with $5 \times 10^{5} \mathrm{~L}$. monocytogenes.............................59 Figure 1.3b. Number of bacterial CFUs in the left lung of rats that were pre-exposed to RTotal, R-Soluble, R-Insoluble, or Saline by intratracheal instillation 3 days prior to intratracheal inoculation with $5 \times 10^{4} \mathrm{~L}$. monocytogenes...........................59 Figure 1.4. Number of bacterial CFUs in the left lung of rats that were pre-exposed to an acidic solution or Saline by intratracheal instillation 3 days prior to intratracheal inoculation with the high dose $\left(5 \times 10^{5}\right)$ of $L$. monocytogenes......................60 Figure 1.5a. Number of bacterial CFUs in the left lung of rats that were pre-exposed to RSoluble, R-Chelex, S-Chelex, or Saline by intratracheal instillation 3 days prior to intratracheal inoculation with $5 \times 10^{5} \mathrm{~L}$. monocytogenes. Figure 1.5b. Number of bacterial CFUs in the left lung of rats that were pre-exposed to RSoluble, R-Chelex, S-Chelex, or Saline by intratracheal instillation 3 days prior to intratracheal inoculation with $5 \times 10^{4} \mathrm{~L}$. monocytogenes...........................61 Figure 1.6. Whole slide scans of hematoxylin and eosin-stained right lung tissue sections from animals intratracheally instilled with R-Soluble, R-Chelex, or Saline followed by inoculation with $5 \times 10^{5}$ L. monocytogenes at days 6 and 10 .

\section{Study 2}

Figure 2.1a. \% change in body weight post-infection of rats that were pre-exposed to RTotal, R-Soluble, R-Chelex, or saline 3 days prior to intratracheal inoculation with $L$. monocytogenes.

Figure 2.1b. Number of bacterial CFUs in the left lungs of rats that were pre-exposed to

R-Total, R-Soluble, R-Chelex, or saline 3 days prior to intratracheal inoculation with $L$. monocytogenes.......................................................... 106 Figure 2.2a. Lactate dehydrogenase (LDH) in the BAL fluid of rats that were pre-exposed to R-Total, R-Soluble, R-Chelex, or saline 3 days prior to intratracheal inoculation with

L. monocytogenes. 
Figure 2.2b. Albumin in the BAL fluid of rats that were pre-exposed to R-Total, RSoluble, R-Chelex, or saline 3 days prior to intratracheal inoculation with $L$.

monocytogenes.

Figure 2.3a. Total cells present in the BAL of rats that were pre-exposed to R-Total, R-

Soluble, R-Chelex, or Saline 3 days prior to intratracheal inoculation with $L$.

monocytogenes as determined by cell differentials............................. 108

Figure 2.3b. Total AMs present in the BAL of rats that were pre-exposed to R-Total, R-

Soluble, R-Chelex, or Saline 3 days prior to intratracheal inoculation with $L$.

monocytogenes as determined by cell differentials.

Figure 2.3c. Total PMNs present in the BAL of rats that were pre-exposed to R-Total, R-

Soluble, R-Chelex, or Saline 3 days prior to intratracheal inoculation with $L$.

monocytogenes as determined by cell differentials.............................. 108

Figure 2.3d. Total lymphocytes present in the BAL of rats that were pre-exposed to R-

Total, R-Soluble, R-Chelex, or Saline 3 days prior to intratracheal inoculation with $L$.

monocytogenes as determined by cell differentials.............................. 108

Figure 2.4a. Total NK cells present in the BAL of rats that were pre-exposed to R-Total,

R-Soluble, R-Chelex, or Saline 3 days prior to intratracheal inoculation with $L$.

monocytogenes as determined by flow cytometry.

Figure 2.4b. Totals T cells present in the BAL of rats that were pre-exposed to R-Total,

R-Soluble, R-Chelex, or Saline 3 days prior to intratracheal inoculation with $L$.

monocytogenes as determined by flow cytometry.

Figure 2.4c. Total CD4+ T cells present in the BAL of rats that were pre-exposed to R-

Total, R-Soluble, R-Chelex, or Saline 3 days prior to intratracheal inoculation with $L$.

monocytogenes as determined by flow cytometry...............................109

Figure 2.4d. Total CD8+ T cells present in the BAL of rats that were pre-exposed to R-

Total, R-Soluble, R-Chelex, or Saline 3 days prior to intratracheal inoculation with $L$.

monocytogenes as determined by flow cytometry.

109

Figure 2.5a. Nitrate and nitrite (NOx) in the BAL fluid from rats that were pre-exposed to

R-Total, R-Soluble, R-Chelex, or Saline 3 days prior to intratracheal inoculation with $L$.

monocytogenes.

.110

Figure 2.5b. Nitrate and nitrite (NOx) in the cell media from BAL cells cultured for 18 hours from rats that were pre-exposed to R-Total, R-Soluble, R-Chelex, or Saline 3 days prior to intratracheal inoculation with $L$. monocytogenes.

Figure 2.6a. BAL cellular chemiluminescence (CL) depicted in total counts per 15 min per $1 \times 10^{6}$ cells after stimulation with PMA in rats that were pre-exposed to R-Total, RSoluble, R-Chelex, or Saline 3 days prior to intratracheal inoculation with $L$.

monocytogenes.

Figure 2.6b. BAL cellular chemiluminescence (CL) depicted in total counts per 15 min per $1 \times 10^{6}$ cells after stimulation with zymosan in rats that were pre-exposed to R-Total, R-Soluble, R-Chelex, or Saline 3 days prior to intratracheal inoculation with $L$.

monocytogenes. .111

Figure 2.7a. Concentration of pro-inflammatory cytokine, TNF- $\alpha$, in the BAL fluid of rats that were pre-exposed to R-Total, R-Soluble, R-Chelex, or Saline 3 days prior to intratracheal inoculation with L. monocytogenes. 
Figure 2.7b. Concentration of acute phase response cytokine, IL-6, in the BAL fluid of rats that were pre-exposed to R-Total, R-Soluble, R-Chelex, or Saline 3 days prior to intratracheal inoculation with $L$. monocytogenes.................................112 Figure 2.8. IL-10, an anti-inflammatory cytokine, in the BAL fluid of rats that were preexposed to R-Total, R-Soluble, R-Chelex, or Saline 3 days prior to intratracheal inoculation with $L$. monocytogenes.

Figure 2.9a. IL-12p70, a cytokine involved in directing the appropriate $\mathrm{T}$ cell response to L. monocytogenes, in the BAL fluid of rats that were pre-exposed to R-Total, R-Soluble, R-Chelex, or Saline 3 days prior to intratracheal inoculation with L. monocytogenes...114 Figure 2.9b. IL-18, a cytokine involved in directing the appropriate T cell response to $L$. monocytogenes, in the BAL fluid of rats that were pre-exposed to R-Total, R-Soluble, RChelex, or Saline 3 days prior to intratracheal inoculation with L. monocytogenes......114 Figure 2.10a. T cell cytokine, IL-2, in the BAL fluid of rats that were pre-exposed to RTotal, R-Soluble, R-Chelex, or Saline 3 days prior to intratracheal inoculation with $L$. monocytogenes.

Figure 2.10b. T cell cytokine, IL-4, in the BAL fluid of rats that were pre-exposed to RTotal, R-Soluble, R-Chelex, or Saline 3 days prior to intratracheal inoculation with $L$. monocytogenes.

\section{Study 3: Part One}

Figure 3.1.1. \% change in body weight post-infection of rats that were pre-exposed to individual soluble metals, the metal mixture, or saline 3 days prior to intratracheal inoculation with $L$. monocytogenes.

Figure 3.1.2a. Lactate dehydrogenase (LDH) in the BAL fluid of rats that were preexposed to individual soluble metals, the metal mixture, or saline 3 days prior to intratracheal inoculation with $L$. monocytogenes...............................168 Figure 3.1.2b. Albumin in the BAL fluid of rats that were pre-exposed to individual soluble metals, the metal mixture, or saline 3 days prior to intratracheal inoculation with

L. monocytogenes.

Figure 3.1.3a. Total cells present in the BAL of rats that were pre-exposed to individual soluble metals, the metal mixture, or saline 3 days prior to intratracheal inoculation with L. monocytogenes

Figure 3.1.3b. Total AMs present in the BAL of rats that were pre-exposed to individual soluble metals, the metal mixture, or saline 3 days prior to intratracheal inoculation with

L. monocytogenes.

Figure 3.1.3c. Total PMNs present in the BAL of rats that were pre-exposed to individual soluble metals, the metal mixture, or saline 3 days prior to intratracheal inoculation with

L. monocytogenes.

Figure 3.1.3d. Total lymphocytes present in the BAL of rats that were pre-exposed to individual soluble metals, the metal mixture, or saline 3 days prior to intratracheal inoculation with L. monocytogenes

Figure 3.1.4a. Total NK cells present in the BAL of rats that were pre-exposed to individual soluble metals, the metal mixture, or saline 3 days prior to intratracheal inoculation with L. monocytogenes. 
Figure 3.1.4b. Total $\mathrm{T}$ cells present in the BAL of rats that were pre-exposed to individual soluble metals, the metal mixture, or saline 3 days prior to intratracheal inoculation with L. monocytogenes......................................................... 170 Figure 3.1.4c. Total CD4+ T cells present in the BAL of rats that were pre-exposed to individual soluble metals, the metal mixture, or saline 3 days prior to intratracheal inoculation with $L$. monocytogenes. ...

Figure 3.1.4d. Total CD8+ T cells present in the BAL of rats that were pre-exposed to individual soluble metals, the metal mixture, or saline 3 days prior to intratracheal inoculation with $L$. monocytogenes. .

Figure 3.1.5. Nitrate and nitrite (NOx) in the BAL fluid (BALF) of rats that were preexposed to individual soluble metals, the metal mixture, or saline 3 days prior to intratracheal inoculation with $L$. monocytogenes...............................171 Figure 3.1.6a. BAL cellular chemiluminescence (CL) depicted in total counts per 15 min per $1 \times 10^{6}$ cells after stimulation with PMA in rats that were pre-exposed to individual soluble metals, the metal mixture, or saline 3 days prior to intratracheal inoculation with

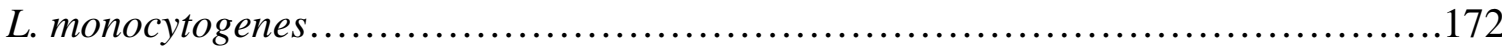
Figure 3.1.6b. BAL cellular chemiluminescence (CL) depicted in total counts per 15 min per $1 \times 10^{6}$ cells after stimulation with zymosan in rats that were pre-exposed to individual soluble metals, the metal mixture, or saline 3 days prior to intratracheal inoculation with L. monocytogenes.

Figure 3.1.7a. Concentration of pro-inflammatory cytokine, TNF- $\alpha$, in the BAL fluid of rats that were pre-exposed to individual soluble metals, the metal mixture, or saline 3 days prior to intratracheal inoculation with L. monocytogenes. Figure 3.1.7b. Concentration of acute phase response cytokine, IL-6, in the BAL fluid of rats that were pre-exposed to individual soluble metals, the metal mixture, or saline 3 days prior to intratracheal inoculation with $L$. monocytogenes.......................173 Figure 3.1.8. IL-10, an anti-inflammatory cytokine, in the BAL fluid of rats that were pre-exposed to individual soluble metals, the metal mixture, or saline 3 days prior to intratracheal inoculation with $L$. monocytogenes.............................. 174 Figure 3.1.9a. IL-12p70, a cytokine involved in directing the appropriate T cell response to L. monocytogenes, in the BAL fluid of rats that were pre-exposed to individual soluble metals, the metal mixture, or saline 3 days prior to intratracheal inoculation with $L$. monocytogenes.......................................................... 175 Figure 3.1.9b. IL-18, a cytokine involved in directing the appropriate $\mathrm{T}$ cell response to L. monocytogenes, in the BAL fluid of rats that were pre-exposed to individual soluble metals, the metal mixture, or saline 3 days prior to intratracheal inoculation with $L$. monocytogenes.

Figure 3.1.10a. T cell cytokine, IL-2, in the BAL fluid of rats that were pre-exposed to individual soluble metals, the metal mixture, or saline 3 days prior to intratracheal inoculation with $L$. monocytogenes.......................................... 176 Figure 3.1.10b. T cell cytokine, IL-4, in the BAL fluid of rats that were pre-exposed to individual soluble metals, the metal mixture, or saline 3 days prior to intratracheal inoculation with $L$. monocytogenes. 


\section{Study 3: Part Two}

Figure 3.2.1. \% change in body weight post-infection of rats that were pre-exposed to soluble metal mixtures, the total metal mixture, or saline 3 days prior to intratracheal inoculation with $L$. monocytogenes..........................................180 Figure 3.2.2a. Lactate dehydrogenase (LDH) in the BAL fluid of rats that were preexposed to soluble metal mixtures, the total metal mixture, or saline 3 days prior to intratracheal inoculation with $L$. monocytogenes.

Figure 3.2.2b. Albumin in the BAL fluid of rats that were pre-exposed to soluble metal mixtures, the total metal mixture, or saline 3 days prior to intratracheal inoculation with

L. monocytogenes. 181

Figure 3.2.3a. Total cells present in the BAL of rats that were pre-exposed to soluble metal mixtures, the total metal mixture, or saline 3 days prior to intratracheal inoculation with $L$. monocytogenes.

Figure 3.2.3b. Total AMs present in the BAL of rats that were pre-exposed to soluble metal mixtures, the total metal mixture, or saline 3 days prior to intratracheal inoculation with $L$. monocytogenes.................................................... 182 Figure 3.2.3c. Total PMNs present in the BAL of rats that were pre-exposed to soluble metal mixtures, the total metal mixture, or saline 3 days prior to intratracheal inoculation with L. monocytogenes.

Figure 3.2.3d. Total lymphocytes present in the BAL of rats that were pre-exposed to soluble metal mixtures, the total metal mixture, or saline 3 days prior to intratracheal inoculation with $L$. monocytogenes.

Figure 3.2.4a. Total NK cells present in the BAL of rats that were pre-exposed to soluble metal mixtures, the total metal mixture, or saline 3 days prior to intratracheal inoculation with L. monocytogenes.

Figure 3.2.4b. Total T cells present in the BAL of rats that were pre-exposed to individual soluble metals, the metal mixture, or saline 3 days prior to intratracheal inoculation with L. monocytogenes.

Figure 3.2.4c. Total CD4+ T cells present in the BAL of rats that were pre-exposed to soluble metal mixtures, the total metal mixture, or saline 3 days prior to intratracheal inoculation with $L$. monocytogenes.

Figure 3.2.4d. Total CD8+ T cells present in the BAL of rats that were pre-exposed to soluble metal mixtures, the total metal mixture, or saline 3 days prior to intratracheal inoculation with $L$. monocytogenes.

Figure 3.2.5. Nitrate and nitrite (NOx) in the BAL fluid (BALF) of rats that were preexposed to soluble metal mixtures, the total metal mixture, or saline 3 days prior to intratracheal inoculation with $L$. monocytogenes................................ 184 Figure 3.2.6a. BAL cellular chemiluminescence (CL) depicted in total counts per 15 min per $1 \times 10^{6}$ cells after stimulation with PMA in rats that were pre-exposed to soluble metal mixtures, the total metal mixture, or saline 3 days prior to intratracheal inoculation with

L. monocytogenes..................................................... 185 Figure 3.2.6b. BAL cellular chemiluminescence (CL) depicted in total counts per 15 min per $1 \times 10^{6}$ cells after stimulation with zymosan in rats that were pre-exposed to soluble metal mixtures, the total metal mixture, or saline 3 days prior to intratracheal inoculation

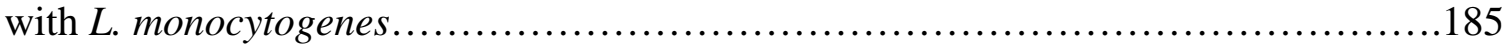


Figure 3.2.7a. Concentration of pro-inflammatory cytokine, TNF- $\alpha$, in the BAL fluid of rats that were pre-exposed to soluble metal mixtures, the total metal mixture, or saline 3 days prior to intratracheal inoculation with $L$. monocytogenes.........................186 Figure 3.2.7b. Concentration of acute phase response cytokine, IL-6, in the BAL fluid of rats that were pre-exposed to soluble metal mixtures, the total metal mixture, or saline 3

days prior to intratracheal inoculation with $L$. monocytogenes.........................186 Figure 3.2.8. IL-10, an anti-inflammatory cytokine, in the BAL fluid of rats that were pre-exposed to soluble metal mixtures, the total metal mixture, or saline 3 days prior to intratracheal inoculation with $L$. monocytogenes..................................187 Figure 3.2.9a. IL-12p70, a cytokine involved in directing the appropriate $\mathrm{T}$ cell response to $L$. monocytogenes, in the BAL fluid of rats that were pre-exposed to soluble metal mixtures, the total metal mixture, or saline 3 days prior to intratracheal inoculation with L. monocytogenes. 188

Figure 3.2.9b. IL-18, a cytokine involved in directing the appropriate $\mathrm{T}$ cell response to L. monocytogenes, in the BAL fluid of rats that were pre-exposed to soluble metal mixtures, the total metal mixture, or saline 3 days prior to intratracheal inoculation with L. monocytogenes. 188

Figure 3.2.10a. T cell cytokine, IL-2, in the BAL fluid of rats that were pre-exposed to soluble metal mixtures, the total metal mixture, or saline 3 days prior to intratracheal inoculation with $L$. monocytogenes

Figure 3.2.10b. T cell cytokine, IL-4, in the BAL fluid of rats that were pre-exposed to soluble metal mixtures, the total metal mixture, or saline 3 days prior to intratracheal inoculation with $L$. monocytogenes. 


\section{LIST OF TABLES}

\section{Study 1}

Table 1.1. Number of animals per treatment group for each time point .63

Table 1.2. Element mass in $\mu \mathrm{g}$ amount present in the $2 \mathrm{mg}$ instillate of the R-Total, RInsoluble, R-Soluble, and R-Chelex samples as determined by inductively coupled argon plasma, atomic-emission spectroscopy....

Table 1.3. \% of total body weight gained by uninfected rats on days 3, 6, 8, and 10 after exposure to Saline, R-Chelex, R-Soluble, or R-Total.

\section{Study 2}

Table 2.1. Ratio of T cells to B cells retrieved from the BAL of rats that were preexposed to R-Total, R-Soluble, R-Chelex, or Saline 3 days prior to intratracheal inoculation with $L$. monocytogenes....................................... 116 Table 2.2. Ratio of CD4+ T cells to CD8+ T cells retrieved from the BAL of rats that were pre-exposed to R-Total, R-Soluble, R-Chelex, or Saline 3 days prior to intratracheal inoculation with $L$. monocytogenes....................................... 117

\section{Study 3: Part One}

Table 3.1.1. Number of bacterial CFUs in the left lungs of rats that were pre-exposed to individual soluble metals, the metal mixture, or saline 3 days prior to intratracheal inoculation with $L$. monocytogenes

Table 3.1.2. Ratio of T cells to B cells retrieved from the BAL of rats that were preexposed to individual soluble metals, the metal mixture, or saline 3 days prior to intratracheal inoculation with $L$. monocytogenes

Table 3.1.3. Ratio of CD4+ T cells to CD8+ T cells retrieved from the BAL of rats that were pre-exposed to individual soluble metals, the metal mixture, or saline 3 days prior to intratracheal inoculation with L. monocytogenes.

\section{Study 3: Part Two}

Table 3.2.1. Quantities of soluble metals present in the $0.3 \mathrm{~mL}$ instillation volume of sterile PBS (vehicle) in each treatment group.

Table 3.2.2. Number of bacterial CFUs in the left lungs of rats that were pre-exposed to soluble metal mixtures, the total metal mixture, or saline 3 days prior to intratracheal inoculation with $L$. monocytogenes

Table 3.2.3. Ratio of $\mathrm{T}$ cells to $\mathrm{B}$ cells retrieved from the BAL of rats that were preexposed to soluble metal mixtures, the total metal mixture, or saline 3 days prior to intratracheal inoculation with $L$. monocytogenes.

Table 3.2.4. Ratio of CD4+ T cells to CD8+ T cells retrieved from the BAL of rats that were pre-exposed to soluble metal mixtures, the total metal mixture, or saline 3 days prior to intratracheal inoculation with $L$. monocytogenes. 


\section{GLOSSARY}

AAALAC - Association for Assessment and Accreditation of Laboratory Animal Care AAD - Amino-actinomycin

$\mathrm{Al}$ - Aluminum

$\mathrm{AlCl}_{3}$ - Aluminum chloride

$\mathrm{Al}_{2} \mathrm{O}_{3}$ - Alumina

$\mathrm{AlNH}_{4}\left(\mathrm{SO}_{4}\right)_{2}-$ Aluminum ammonium sulfate

$\mathrm{Al}_{2}\left(\mathrm{SO}_{4}\right)_{3}$ - Aluminum sulfate

AM - Alveolar macrophage

ANOVA - Analysis of variance

APC - Antigen presenting cell

ATS - American Thoracic Society

ATSDR - Agency for Toxic Substances and Disease Registry

$\mathrm{Ba}$ - Barium

BAL - Bronchoalveolar lavage

BALF - Bronchoalveolar lavage fluid

Be - Beryllium

bw - body weight

$\mathrm{Ca}$ - Calcium

CAPs - Concentrated ambient particles

CAR - Cilia-associated respiratory

Cd - Cadium

CD - Cluster of differentiation

CFA - Coal fly ash

$\mathrm{CFU}$ - Colony forming unit

$\mathrm{CL}$ - Chemiluminescence

$\mathrm{Cl}_{2}$ - Chloride

Co - Cobalt

COPD - Chronic obstructive pulmonary disease

cpm - counts per minute

$\mathrm{Cr}$ - Chromium

$\mathrm{Cu}$ - Copper

CVF - Certified virus-free

CWP - Coal workers' pneumoconiosis

DC - Dendritic cell

DNA - Deoxyribonucleic acid

ELISA - Enzyme-linked immunosorbent assay

EMEM - Eagle's minimum essential medium

EPA - Environmental Protection Agency

ESR - Electron spin resonance

FACS- Flourescence-activated cell sorter

$\mathrm{Fe}$ - Iron

$\mathrm{Fe}^{2+}-$ Ferrous iron

$\mathrm{Fe}^{3+}-$ Ferric iron 
$\mathrm{FeCl}_{2}-$ Ferrous chloride

$\mathrm{FeCl}_{3}-$ Ferric chloride

$\mathrm{FeNH}_{4}\left(\mathrm{SO}_{4}\right)_{2}-$ Ferric ammonium sulfate

$\mathrm{Fe}_{2} \mathrm{O}_{3}$ - Ferric oxide

$\mathrm{FeSO}_{4}$ - Ferrous sulfate

$\mathrm{Fe}_{2}\left(\mathrm{SO}_{4}\right)_{3}-$ Ferric sulfate

$\mathrm{H} \& \mathrm{E}$ - Hematoxylin and eosin

$\mathrm{H}_{2} \mathrm{SO}_{4}$ - Sulfuric acid

HKL - Heat-killed Listeria

IARC - International Agency for Research on Cancer

Ig - Immunoglobulin

IL - Interleukin

INF - Interferon

iNOS - inducible nitric oxide synthase

I.T. - Intratracheal instillation

L-NAME - $N^{\mathrm{G}}$-nitro-L-arginine methyl ester

L-NMMA - $N^{\mathrm{G}}$-monomethyl-L-arginine

LDH - Lactate dehydrogenase

LPS - Lipopolysaccharide

$\mathrm{MCP}$ - Monocyte chemotactic protein

MIP - Macrophage inflammatory protein

MHC - Major histocompatability complex

Mn - Manganese

NAAQS - National ambient air quality standards

NAD+ - Nictotinamide adenosine dinucleotide

N.D. - Not determined

$\mathrm{Ni}$ - Nickel

$\mathrm{NiCl}_{2}$ - Nickel chloride

NiO- Nickel oxide

NiS - Nickel sulfide

$\mathrm{Ni}_{3} \mathrm{~S}_{2}-$ Nickel subsulfide

$\mathrm{NiSO}_{4}-$ Nickel sulfate

NIOSH - National Institute for Occupational Safety and Health

NK - Natural killer cell

$\mathrm{NO}$ - Nitric oxide

$\mathrm{NO}_{3}$ - nitrate

NOS - Nitric oxide synthase

NOx - Nitrate and nitrite

NTP - Natioanl Toxicology Program

PAH - Polycyclic aromatic hydrocarbon

$\mathrm{Pb}$ - Lead

PBS - Phosphate buffered saline

PM -Particulate matter

PMA - Phorbol 12-myristate 13-acetate

PMN - Polymorphonuclear leukocytes

RNA - Ribonucleic acid 
RNS - Reactive nitrogen species

ROFA - Residual oil fly ash

ROS - Reactive oxygen species

RSV - Respiratory synsytial virus

S - Sulfur

SD - Sprague-Dawley

SE - Standard error

$\mathrm{SO}_{4}$ - Sulfate

$\mathrm{T}_{\mathrm{C}}-$ Cytotoxic $\mathrm{T}$ cell

$\mathrm{T}_{\mathrm{H}}-$ Helper $\mathrm{T}$ cell

$\mathrm{Ti}$ - Titanium

TNF - Tumor necrosis factor

UV - Utah Valley

UVD - Utah Valley Dust

$\mathrm{V}$ - Vandium

$\mathrm{VCl}_{2}$ - Vanadium chloride

$\mathrm{V}_{2} \mathrm{O}_{5}$ - Vanadium pentoxide

$\mathrm{VOSO}_{4}$ - Vanadyl sulfate

WHO - World Health Organization

$\mathrm{Zn}-$ Zinc

$\mathrm{ZnCl}_{2}$ - Zinc chloride

$\mathrm{ZnO}$ - Zinc oxide

$\mathrm{ZnS}$ - Sphalerite

$\mathrm{ZnSO}_{4}-\mathrm{Zinc}$ sulfate 


\section{LITERATURE REVIEW}

\section{$\underline{\text { Air Pollutant Particulate Matter and Associated Health Effects }}$}

In Donora, PA, in 1948, and London, England, in 1952, there were two extreme and publicly recognized air pollution episodes that were directly associated with significantly increased morbidity and mortality (Bell and Davis, 2001; Ministry of Health, 1954; Shrenk et al., 1949; Wilkins, 1952). In both instances, meteorologic conditions brought on the events that led to thick smog settling in the cities, and local iron and steel industries, metal works, coke ovens and coal-fired furnaces contributed to the high levels of airborne particulates. In Donora, the death rate was 6-times the average for that time of year, and in London, 3000 to 4000 deaths were directly associated with the episode, and thousands of deaths above the average in the next 3 months were likely attributed to the London smog. Events such as these have led to the adoption of air quality standards that are in effect today for a number of air pollutants including particulate matter (PM).

PM is chemically complex. The major components include carbon, sulfates, and nitrates, as well as crustal species such as soil and ash, and the sources of origin are both anthropologic and natural (EPA, 2004). PM is currently divided into two regulatory categories based on the aerodynamic diameter of the particles. $\mathrm{PM}_{10}$ refers to particles that range in diameter equal to or smaller than $10 \mu \mathrm{m}$, which are considered to be "inhalable" and can penetrate the respiratory tract, and include coarse particles $(2.5 \mu \mathrm{m}-$ $10 \mu \mathrm{m}$ in diameter). $\mathrm{PM}_{2.5}$ particles are categorized as fine or ultrafine with diameters equal to or smaller than $2.5 \mu \mathrm{m}$. The National Ambient Air Quality Standards (NAAQS) for $\mathrm{PM}_{10}$ are $150 \mu \mathrm{g} / \mathrm{m}^{3}$ for the short-term (24 hour) average, and $50 \mu \mathrm{g} / \mathrm{m}^{3}$ for the long- 
term (annual) average. The short-term and long-term standards for $\mathrm{PM}_{2.5}$ are $65 \mu \mathrm{g} / \mathrm{m}^{3}$ and $15 \mu \mathrm{g} / \mathrm{m}^{3}$, respectively (EPA, 1997). Industrial processes, vehicle emissions, and fuel combustion contribute significant quantities to both categories, with a particularly large amount of the $\mathrm{PM}_{2.5}$ coming from combustion sources (EPA, 2004). The estimated 2004 PM emissions for $\mathrm{PM}_{10}$ and $\mathrm{PM}_{2.5}$, excluding secondary $\mathrm{PM}$ formed from condensation of primary emissions in the atmosphere, were 2.5 and 1.9 million tons, respectively (EPA, 2005). In 2003, 718 thousand tons of the $\mathrm{PM}_{2.5}$ emissions (excluding condensibles) were attributed to fuel combustion, with 139 thousand tons of fuel combustion emissions coming from coal- and oil-burning power generating plants combined (EPA, 2005).

Long term and large cohort epidemiological studies have shown a correlation between PM exposure and elevated morbidity and mortality indicated by increased hospital emissions for both cardiovascular and respiratory diseases (Abbey et al., 1999; Dockery et al., 1993; Englert, 2004; Ghio, 2004; Pope et al., 1995; 2004; Samet et al., 2000a, 2000b; Schwartz, 1994). In addition, studies across the United States and around the world indicate that acute exposure to increased levels of PM air pollution can cause adverse respiratory effects, exacerbation of existing pulmonary and cardiological disease, and increased morbidity and mortality as well (ATS, 1996; Borja-Aburto et al., 2004; Chen et al., 2004; Dockery and Pope, 1994; Holgate et al., 2003; Samet et al., 2000a; Schwartz, 1994; Schwartz et al., 2001; WHO, 2003; Wichmann et al., 2000). According to these studies, conditions regarding pulmonary effects include, but are not limited to, increased mortality from pneumonia and influenza, increased airway reactivity and exacerbations in asthma, increased respiratory symptoms (cough, wheeze), decreased 
lung function, increased lung inflammation, and altered pulmonary host defense (ATS, 1996).

\section{Effects of PM on Pulmonary Host Defense}

An epidemiological study re-analysis by Pope et al. (2004) showed that the leading cause of mortality in response to increases in ambient air PM in the category of pulmonary conditions was infection, including pneumonia and influenza. A number of other epidemiological cohort studies, and in vivo and in vitro studies, have examined alterations in immune parameters in human, animal, and cellular systems in response to PM, which may be indicative of increased susceptibility to infection following PM exposure. Nobutomo (1978) found that sputum from individuals in an industrial area had increased phagocytes and lymphocytes, and that this increase could be correlated to the onset of bronchitis. Population studies in industrialized areas have also revealed that in regions with high anthropologic $\mathrm{PM}$ loads, particularly in the $\mathrm{PM}_{2.5}$ range, whole blood and serum levels of lymphocytes (natural killer (NK) cells, B and T cell subsets), immunoglobulin (Ig), and cytokines are significantly altered (Calderon-Garciduenas et al., 2000; Hertz-Picciotto et al., 2005; Leonardi et al., 2000; Skachkova et al., 2001). Human exposure studies have also demonstrated that PM that is high in metals can increase leukocytes in the lung, as well as increase oxidant and cytokine production by phagocytes (Ghio, 2004; Ghio et al., 2000; Schaumann et al., 2004), i.e., cellular processes that are important in pulmonary responses to pathogens.

To address whether these particles alter infectivity and pulmonary host defense more directly, animal models have been employed to determine the effects of various air pollutants on lung responses to pathogens, as well as the ability of particulates to 
exacerbate existing respiratory conditions. The epidemiology studies above indicate that morbidity associated with PM is more detrimental to individuals with pre-existing cardiopulmonary conditions, such as chronic obstructive pulmonary disease (COPD). A common form of COPD is chronic bronchitis. Pulmonary exposure to concentrated ambient air particulates (CAPs) in rodent models of chronic bronchitis has demonstrated exacerbation of the condition, with increased inflammation, neutrophil influx into the lungs, and increased indices of tissue damage in lung fluids, when compared to unexposed bronchitic rats and non-bronchitic rats that received only CAPS (Clarke et al., 1999; Kodavanti et al., 2000). In addition, Zelikoff et al. (2003) found that $\mathrm{PM}_{2.5}$ collected from New York City increased lung bacterial burdens in previously infected rats, worsening their condition.

Pre-exposure to air pollutants can also enhance susceptibility to pulmonary infection. Exposure to ozone decreased bacterial clearance from the lungs of rats (Cohen et al., 2002; Steerenberg et al., 2004). Pulmonary exposure to diesel exhaust prior to infection with a virus (Hahon et al., 1985; Harrod et al., 2003), or a bacterial pathogen (Harrod et al., 2005; Yang et al., 2001; Yin et al., 2002; 2003), increased pulmonary infection in rodents, altering both innate and adaptive immune responses. In a cellular system, macrophages pre-exposed to PM collected in Ottawa, followed by culture with respiratory syncytial virus (RSV)-infected epithelial cells, led to increased viral load, and decreased uptake and killing by macrophages (Becker and Soukup, 1999). Residual oil fly ash (ROFA) (Antonini et al., 2002; Hatch et al., 1985), certain types of coal fly ash (CFA) (Hatch et al., 1985), copper smelter fly ash (Aranyi et al., 1981), and various CAPs (Hatch et al., 1985) have been shown to reduce the clearance of bacteria from the 
lungs of rats and increase bacterial infectivity. Interestingly, Hatch et al. (1985) found that the ashes and particulates that were most potent at altering infectivity were the samples that were smallest in size and/or highest in soluble metal content.

The cellular mechanisms by which the alterations in lung host defense take place in response to pollutant exposure are not fully understood, and may differ based on the PM composition. A plethora of studies exist that have examined effects of various forms of PM on a number of different pulmonary cell types in the lung, more so in the absence of infection, including cells that are involved in innate (alveolar macrophages (AMs), neutrophils, dendritic cells (DCs), NK cells) and adaptive (T and B lymphocytes) cellular immune responses. Macrophage responses to PM have been widely investigated and include, but are not limited to, alterations in phagocytic ability (Antonini et al., 2002; Yin et al., 2002; Goldsmith et al, 1997; Hadnagy and Seemayer, 1994), receptor expression (Becker et al., 2002b; Becker and Soukup, 2003), antigen-presenting ability (Hamilton et al., 2004), and production of oxidants (Goldsmith et al., 1997; Kleinman et al., 2003; Lewis et al., 2003; Tao et al., 2003; Yin et al., 2004b) and cytokines (Becker et al., 1996; Dorger and Krombach, 2002; Dye et al., 1999; Carter et al., 1997; Yin et al., 2002; 2003). Neutrophil responses were generally found to be enhanced after exposure to PM, including increased influx into the lungs and circulation, and increased oxidant production and cytokine production (Antonini et al., 2002; Hippeli and Elstner, 1990; Hitzfeld et al., 1997; Huang et al., 2003b; Kodavanti et al., 1998; 2000; Krishna et al., 1998; Yin et al., 2002; Yokota et al., 2005). Direct responses of DCs and NK cells to PM are not as well investigated. Ohtani et al. (2005) showed that diesel exposure to DCs inhibited production of antigen-presenting cytokines while stimulating inflammatory 
cytokines. Ozone appears to suppress the activity of NK cells in the lung (Burleson et al., 1989), while exposure to CFA caused a slight increase in the number of NK cells present in the lungs of rats (Dormans et al., 1999). Lymphocyte responses to PM include increased infiltration into the lung (Clarke et al., 1999; Dormans et al., 1999; Lambert et al., 1999; van Zijverden et al., 2000; Yin et al., 2003), and alteration in proliferation (Omara et al., 2000), porphyrin metabolism (Muzyka et al., 2003), Ig expression (Dormans et al., 1999; Lambert et al., 1999; van Zijverden et al., 2000) and cytokine production (Drela et al., 2002; van Zijverden et al., 2000; Yin et al., 2002; 2003).

In the studies mentioned above, there are variable effects for certain cell types, such as macrophages, which may be attributable to the dose or composition of PM, as well as the species of animal or cell line that was investigated. In some instances the effects of the PM are stimulatory, whereas in others the cellular responses may be inhibited. For example, Goldsmith et al. (1997) showed CAPs to increase phagocytosis and oxidant production by macrophages, whereas others have shown decreased phagocytsosis (Hadnagy and Seemayer, 1994) and decreased oxidant production (Kleinman et al., 2003) in response to ambient particles. The study by van Zijverden et al. (2000) showed differential responses of T lymphocyte subsets after exposure to carbon black, silica, or diesel. Therefore, differences in PM dose, source and composition are the likely explanation for the variety of effects observed for a given cell type in the lung.

\section{Role of Transition Metals in $\mathbf{P M}_{2.5}$ Toxicity}

There is mounting evidence that particle size and composition are important determinants in responses to PM. Epidemiological studies have shown that $\mathrm{PM}_{2.5}$ correlates better with mortality and morbidity than $\mathrm{PM}_{10}$ (Schwartz et al., 1996; 
Wichmann et al., 2000). Because a large portion of the $\mathrm{PM}_{2.5}$ burden comes from combustion sources, there is good indication for a strong involvement for vehicle - and power plant- derived pollutants in adverse health effects (EPA, 2004). Another strong factor in determining particle toxicity, and consequently public health, appears to be particle composition. Analysis of data from a Canadian epidemiological study by Burnett et al. (2000) showed that iron (Fe), sulfate, nickel (Ni) and zinc ( $\mathrm{Zn})$ in ambient $\mathrm{PM}_{2.5}$ correlated more strongly with mortality than $\mathrm{PM}_{2.5}$ mass. Using the data generated from the Harvard Six Cities Study (Dockery et al., 1993; Ferris et al., 1979; Spengler and Thurston, 1983), Laden et al. (2000) used metals that were determined to be characteristic of various emissions sources to further define correlations between source-related fractions of fine particles and mortality. In this study, the authors found that the fine particles from oil combustion had the closest correlation to mortality, followed by coal combustion, then mobile sources. In fact, analysis of lung tissue archived from the fatalities from COPD or congestive heart failure during the London Smog incident showed aggregations of fine carbonaceous matter associated with a variety of fine, inorganic material, which consisted primarily of selenium $(\mathrm{Se}), \mathrm{Fe}$, lead $(\mathrm{Pb}), \mathrm{Zn}$, and, to a lesser extent, copper (Cu), manganese (Mn), cadmium (Cd), and Ni (Hunt et al., 2003), i.e., metals associated with fuel combustion. Another epidemiologic study showed that there were increased incidences of asthma and bronchitis in children in a German smelter area (Heystedt) when compared to a less industrialized area (Zerbst) (Heinrich et al., 1999). Schaumann et al. (2004) treated human volunteers with the $\mathrm{PM}_{2.5}$ from these two areas and found that the dust from the more industrialized town, which had significantly 
more metals $(\mathrm{Zn}, \mathrm{Cu}, \mathrm{Fe}$, and $\mathrm{Ni})$, caused increased inflammation and generation of oxidant radicals when compared to the $\mathrm{PM}_{2.5}$ from the non-industrial region.

PM collected from the Utah Valley (UV) offered insight into the effects of PM with varying metal concentrations (Dye et al., 2001; Ghio, 2004; Ghio et al., 1999; Molinelli et al., 2002; Soukup et al., 2000). The primary contributor to PM in the area was a steel mill, and dust (UVD) was collected prior to its closure, during the period of time that the mill was shut down, and after reopening. During the closure, the soluble extract of the PM was relatively low in metal concentration, while before closure and after reopening the concentrations of $\mathrm{Zn}$ and $\mathrm{Cu}$ were 30-60 times higher. There were also significantly greater amounts of $\mathrm{Pb}$ and $\mathrm{Fe}$, and, to a lesser degree, $\mathrm{Ni}$ and vanadium (V). In vivo studies in humans and rodents, as well as in vitro studies, showed increased airway responsiveness and inflammation, increased oxidant production, and altered alveolar macrophage (AM) function in response to the UVD with high metal concentrations when compared to that of the lower concentration (Dye et al., 2001; Ghio et al., 1999; Molinelli et al., 2002; Soukup et al., 2000).

Metals also appear to be a major determinant in the toxicity of PM derived from the combustion of fossil fuels. The metal fraction of ROFA has been shown, in both in vivo and in vitro studies, to increase lung injury and cytotoxicity (Dreher et al., 1997; Dye et al., 1999; Gavett et al., 1997; Kodavanti et al., 1998; 2001), increase lung inflammation (Kodavanti et al., 1998; 2001), increase airway reactivity (Gavett et al., 1997; Lambert et al., 2000), alter cytokine production (Dye et al., 1999; Carter et al., 1997), and increase oxidative stress (Ghio et al., 2002b; Kim et al., 2003; Lewis et al., 2003). The majority of these studies demonstrate that the responses are due primarily to 
the increased bioavailability of the soluble metals in the extracts of the samples. Bioavailability of Fe in CFA has also been shown to increase production of reactive oxygen species, which may increase oxidative stress in the lung (Ball et al., 2000), and soluble sulfur (S), Zn, and V were associated with increased lung injury and permeability (Fernandez et al., 2003). Soluble metal extracts of other ambient air particulate samples have also been shown to produce pulmonary toxicity (Ghio, 2004; Huang et al., 2003a; Prieditis and Adamson, 2002). Costa and Dreher (1997) tested PM from 3 different emissions sources ( 2 from oil and 1 from coal combustion) and 4 ambient air samples from different locations (St. Louis, MO; WA; Dusseldorf, Germany; Ottowa, Canada). The authors found that in all samples the bioavailable metal extracts were the primary determinants of pulmonary inflammation in vivo when compared to PM mass on an equimass versus equi-metal basis.

The effects of soluble metals in PM on the lung described above may result in increased susceptibility to infection. Studies have implicated the soluble forms of some metals that exist in PM, primarily sulfates or chlorides, in increased susceptibility to bacterial infection. Such metals include $\mathrm{Zn}$, aluminum $(\mathrm{Al}), \mathrm{Cd}, \mathrm{Cu}$, ferric iron $\left(\mathrm{Fe}^{3+}\right)$, and Ni (Ehrlich, 1980; Hatch et al., 1981; 1985; Zelikoff et al., 2002). Whether or not these effects belong solely to the soluble portion of PM is not clear. Carbon black alone, as a core component in PM, does not appear to alter clearance of bacteria from the lungs of rodents (Adkins et al., 1980; Yang et al., 2001). However, the particulate oxide forms of the metals, including ferrous iron $\left(\mathrm{Fe}^{2+}\right)$ and $\mathrm{Zn}$ (Hatch et al., 1985), and $\mathrm{Fe}^{3+}$ (Aranyi et al., 1983), also may alter immune responses to bacteria. The data concerning Fe is more controversial as studies have also shown that iron oxide $\left(\mathrm{Fe}_{2} \mathrm{O}_{3}\right)$ (Adkins et al., 
$1980)$ and ferrous sulfate $\left(\mathrm{FeSO}_{4}\right)$ (Goldstein et al., 1979) did not alter bacterial clearance from the lungs or rodents.

The epidemiological data and in vivo and in vitro data discussed above suggests that PM categorized as fine and high in metal content may be largely responsible for adverse health effects, such as increased incidence of pulmonary infection. The size and composition characteristics, particularly metal content, of PM suggest that particulates from combustion-derived sources may be significantly associated with these health effects. Because of its size and metal content, ROFA has been used as a model to investigate the toxicity associated with combustion-derived PM.

\section{$\underline{\text { Residual Oil Fly Ash (ROFA) }}$}

Fly ash is defined as the portion of ash produced during fuel combustion that is small enough in size to be emitted and carried away from the boiler in flue gas (ElMogazi et al., 1988; Ghio et al., 2002a). ROFA is a particulate pollutant derived from the combustion of fossil fuel by oil burning power plants and other facilities that use residual oil as a main fuel source. ROFA, like many particles from anthropologic sources, is a fine particulate, with a mean aerodynamic diameter of less than $2.5 \mu \mathrm{m}$, and is primarily inorganic. The particles are a complex mixture of metals (both soluble and insoluble), sulfates, acids, and other unknown material complexed to an insoluble carbonaceous core (Antonini et al., 2004a; Ghio et al., 2002a; Kodavanti et al., 1998; Schroeder et al., 1987). Fuel source, combustion conditions, emissions abatement technology, and byproduct processing all contribute to the properties of ROFA (El-Mogazi et al., 1988). In addition, the metals and metal solubility can vary between power plants and within a plant or facility based on the collection site (Antonini et al., 2004a; Kodavanti et al., 1998). 
Metals present in ROFA can include Fe, V, Ni, Al, Zn, calcium (Ca), and to a lesser extent, $\mathrm{Cu}$, cobalt $(\mathrm{Co}), \mathrm{Cd}$, barium $(\mathrm{Ba})$, beryllium $(\mathrm{Be})$, manganese $(\mathrm{Mn})$, chromium (Cr), and Pb (Antonini et al., 2002; Ghio et al., 2002a; Kodavanti et al., 1998).

ROFA is an environmental and an occupational hazard. As mentioned above, combustion of fossil fuel contributes significantly to the ambient air $\mathrm{PM}_{2.5}$ particulate burdens which have been associated with increased morbidity and mortality. Occupational exposure levels have been reported as high as $6.7 \mathrm{mg} / \mathrm{m}^{3}$ (Hauser et al., 1995). Because of its size, which allows it to remain airborne for a considerable time and renders it almost entirely respirable (Dreher et al., 1997; Natusch et al., 1974; Watson et al., 1995), ROFA has become a model particulate in addressing pulmonary toxicity in relation to PM, and particularly for evaluation of the effects of metal components in PM.

Because ROFA is only one constituent of ambient air PM, it is difficult to attribute the specific effects of environmental PM exposure in the general population directly to ROFA. However, there have been a number of studies on boilermakers after occupational exposure to ROFA. These workers tend to have a history of chronic bronchitis or asthmatic bronchitis known as "boilermakers' bronchitis" (Hauser et al., 1995a; 1995b; 2002; Levy et al., 1984) which results in decreased lung function and airway obstruction. Boilermakers exposed to high ash concentrations have a history of eye irritation, cough, wheeze, dyspnea, and in rare instances pneumonitis, with the majority of the effects attributed to vanadium pentoxide $\left(\mathrm{V}_{2} \mathrm{O}_{5}\right)$ content in ash (Ghio et al., 2002a; Hauser et al., 2002; Sjorberg, 1955; Williams, 1952). In more recent studies of boilermakers, expired nitric oxide (NO) (Kim et al., 2003), indicators of oxidative DNA damage (8-hydroxy-2'-deoxyguanosine) in the urine (Kim et al., 2004; Mukherjee et al., 
2005), and polycyclic aromatic hydrocarbon (PAH)-exposure biomarkers in urine (Mukherjee et al., 2005) have been positively associated with metals that are also present in urine, indicating metal exposure by these workers may put them at risk for increased oxidative DNA damage and adverse respiratory effects. Hauser et al. (1995b) have also shown that non-smoker boilermakers have an increased incidence of airway inflammation, as indicated by elevated neutrophils in nasal lavage. However, direct effects of fly ash on boilermakers can be difficult to conclude as they are often exposed to other agents such as welding fumes or asbestos. Animal and cell system models of ROFA exposure have provided more specific insight into some of the pulmonary effects resulting from ROFA exposure.

In vivo animal models have shown that ROFA exposure leads to a number of pulmonary effects, including increased lung injury, inflammation, and cytotoxicity (Costa and Dreher, 1997; Dreher et al., 1997; Kodavanti et al., 1998; 2001), airway hyperreactivity and enhanced sensitization to allergens (Gavett et al., 1999; Goldsmith et al., 1999; Hamada et al., 2002; Lambert et al., 2000) and susceptibility to infection (Antonini et al., 2002; Hatch et al., 1985). In addition, respiratory exposure to ROFA also results in hematological effects (Gardner et al., 2000; Medeiros et al., 2004), and cardiovascular (Campen et al., 2001; 2002; Costa and Dreher, 1997; Watkinson et al., 1998) and microvascular effects (Nurkiewicz et al., 2004). In terms of lung injury, inflammation, and hyperreactivity, in vivo and in vitro studies suggest mechanisms by which some of these effects may occur, demonstrating possible roles for (1) changes in intracellular signaling and gene transcription (Nadadur and Kodavanti, 2002; Nadadur et al., 2000; Roberts et al., 2004; Samet et al., 1997; 2002; Silbajoris et al., 2000), (2) 
increased oxidative stress (Becker et al., 2002b; Dye et al., 1997; Ghio et al., 2002b;

Goldsmith et al., 1997; 1998; Hamada et al., 2002; Huang et al., 2003a; Jiang et al., 2000;

Kadiiska et al., 1997; Lewis et al., 2003; Prahalad et al., 2001), (3) altered cytokine

production (Carter et al., 1997; Dye et al., 1999; Gao et al., 2004; Lambert et al., 2000;

Lindroos et al., 1997; Roberts et al., 2003; van Eeden et al., 2001; Veronesi et al., 1999),

(4) increased prostaglandin production (Samet et al., 1996) and (5) alterations in receptor

expression/activity on various cell types including lung epithelial cells and macrophages

(Becker et al., 2002a; Gilmour et al., 2004; Goldsmith et al., 1997; Hollingsworth et al., 2004; Huang et al., 2002a; Lindroos et al., 1997; Veronesi et al., 1999; 2002). It is

possible that these mechanisms may also play a role in enhanced susceptibility to infection after ROFA exposure (Antonini et al., 2002; Hatch et al., 1985); however, there are few studies to date that examine the effects of ROFA or its components on susceptibility to infection, and results with different bacteria are conflicting (Antonini et al., 2002; Gilmour et al., 2001).

As discussed in the previous section, a number of studies indicate that the metal component, primarily the soluble metals, of ROFA may be responsible for much of its toxic effects. Dreher et al. (1997) showed that ROFA produced a severe inflammation in the lungs of rats that included increased neutrophil, eosinophil, and monocyte influx into the lungs, as well as increased levels of lactacte dehydrogenase (LDH) and albumin, indices of increased lung damage and cytotoxicity. The degree of lung injury and inflammation was replicated by the water-leachable portion of the ROFA sample which was found to be high in $\mathrm{Fe}, \mathrm{Ni}, \mathrm{V}, \mathrm{Ca}, \mathrm{Mg}$, and sulfate $\left(\mathrm{SO}_{4}\right)$, but not with the insoluble portion of the sample. In addition, depletion of the metals from the soluble sample 
resulted in a less severe injury to the lungs, indicating that the soluble metal portion of the sample was the primary constituent responsible for the response.

Using ROFA samples from various parts of a power plant with varying amounts of water-leachable metals, Kodavanti et al. (1998) also demonstrated that the ROFA with high soluble metal content was more effective at inducing lung injury and inflammation than samples with low soluble metal composition. Antonini et al. (2004a), also examined the ability of oil fly ash from different parts of a power plant to generate pulmonary inflammation in rats, as well as whether the particles could generate free radical intermediates as detected by electron spin resonance (ESR). The precipitator ROFA was more acidic and more soluble than the air heater ROFA, and possessed a greater mass of each metal when compared to the air heater sample. A prominent hydroxyl radical signal was associated with the precipitator ROFA and this could be attributed entirely to the soluble metal fraction of the ROFA, whereas ROFA from the air heater did not produce a radical signal. In addition, the precipitator ROFA produced significant pulmonary inflammation and enhanced susceptibility to lung infection when compared to the air heater sample. These studies indicate that the metal component and the metal solubility of ROFA are important in the pulmonary toxicity associated with ROFA.

In addition to lung injury and inflammation, the soluble metals of ROFA appear to participate in airway hyperreactivity and allergic sensitization (Gavett et al., 1997; Ghio et al., 2002a; Lambert et al., 2000), increased mucin secretion (Jiang et al., 2000), increased oxidant production (Goldsmith et al., 1998; Huang et al., 2003; Kadiiska et al., 1997), altered cytokine expression (Carter et al., 1997; Dye et al., 1999; Gao et al., 2004; Lambert et al., 2000), altered signal transduction (Samet et al., 1997), and gene 
expression (Kodavanti et al., 1997; Nadadur and Kodavanti, 2002). Investigators have also tried to determine which metal or metal combination in the soluble component of ROFA may be responsible for a given effect. Dreher et al. (1997) showed that a surrogate solution of $\mathrm{Fe}, \mathrm{Ni}$, and $\mathrm{V}$ as sulfates could largely reproduce the pulmonary inflammation and injury that they had observed with the ROFA and its soluble component. In addition, they found that $\mathrm{Ni}$ alone was the most toxic and produced a more progressive injury at later time points post exposure. When combined with $\mathrm{Fe}^{2+}$, the toxicity of $\mathrm{Ni}$ was not affected; however, in combination with $\mathrm{V}^{2+}$ or $\mathrm{Fe}^{3+}$, the toxicity of Ni was actually attenuated. These findings suggest a complexity of metal-metal interactions that may exist in the soluble portion of ROFA. Kodavanti et al. (1997) also found that a combination of $\mathrm{Fe}, \mathrm{V}$, and $\mathrm{Ni}$ could reproduce the lung injury observed with ROFA, although to a lesser degree. Ni produced a more severe pulmonary pathology than Fe and $\mathrm{V}$, and $\mathrm{Ni}$ alone was responsible for the alveolar hyperplasia that was observed in the animals. The metal combination also resulted in a similar cytokine response as observed with ROFA, and this elevation in cytokines persisted longest after exposure to ROFA or Ni alone. Kodavanti et al. (1998) also showed that increased LDH and protein in the lungs of rats correlated best with ROFA that was high in $\mathrm{Ni}$ and $\mathrm{Fe}$, whereas macrophage oxidant production and neutrophil increases correlated better with V content. In 2002, the authors also indicated that $\mathrm{ZnSO}_{4}$ could cause a comparable inflammatory response when compared to that observed with ROFA that contained high levels of $\mathrm{Zn}$ in the leachable portion. Also, a number of researchers have attributed the production of oxidants to the $\mathrm{V}$ component of ROFA, and that application of oxidant scavengers to the various 
experimental systems, the effects of ROFA or V were reversed (Ghio et al., 2002; Huang et al., 2003; Kadiiska et al., 1997).

Cytokine production appears to be more complicated. Some researchers have attributed the cytokine production induced by ROFA to its V content (Carter et al., 1997; Dye et al., 1999), others indicate Ni as a strong candidate (Gao et al., 2004), and still others show a role for Fe in differential cytokine expression (Lambert et al., 2000). Cytokine production is likely altered in response to a number of metals and may depend on the cell system, metal, metal concentration, or cytokine of interest, and may be altered by metal-metal interactions. Kodavanti et al. (1997) showed that Fe, V, and Ni could alter cytokine responses individually, although to varying degrees, and Nadadur and Kodavanti (2002) found that gene expression of different cytokines also depended on the metals.

Cytokines, as cell signaling factors, and oxidants, as cell signaling and antimicrobial agents, play critical roles in responses to pulmonary infection. Effects of soluble metals such as these may ultimately affect the pulmonary immune response to pathogens after exposure to ROFA. In regards to ROFA exposure, there is limited information as to the effects of the soluble portion on respiratory host defense in response to challenge with a pathogen. The effects of the metal-soluble fraction of ROFA on some of the cells involved in immune responses in the absence of infection have been examined. Researchers have shown that macrophage function can be altered (Goldsmith et al., 1998; Kodavanti et al., 1998), and that the metals may have antagonistic effects, where $\mathrm{V}$ may be stimulatory but Ni may be inhibitory. How these metals exert their effects when in a mixture, such as the soluble fraction of ROFA, and how this may alter 
susceptibility to infection has not been shown. There is evidence that the soluble fraction of ROFA can alter immune cell responses in an animal model of allergic sensitization (Lambert et al., 2000). In this study, the authors found that Ni was most effective at inducing bronchoconstriction, similar to that observed with ROFA, after antigen challenge. They also found that the IgE production and lymphocyte proliferation in response to the antigen challenge that was enhanced by the soluble portion of ROFA could also be produced by $\mathrm{Ni}$ and $\mathrm{V}$, and that Fe appeared to be primarily responsible for the increases in interleukin-10. It appears that soluble metals in ROFA can have effects on the activity of the cells involved in immune response to pulmonary infection, although how these responses may actually be altered during the course of an infection after exposure to the soluble metal of ROFA has not been shown.

\section{Pulmonary Immunotoxicology of $\mathrm{Al}, \mathrm{Fe}, \mathrm{Ni}, \mathrm{V}$, and $\mathrm{Zn}$}

Occupational and environmental respiratory exposure to various metals can result in a number of parenchymal diseases (inorganic dust-pneumonia, granulomatous disease, pneumonitis, interstitial fibrosis), airway disorders, and cancers (Brooks, 1981; Cohen et al., 2004; Kelleher et al., 2000). In addition, certain metals can exert immunotoxicologic effects in the lungs after inhalation, and in some instances, after non-respiratory exposures (Cohen et al., 2004). Therefore, it is plausible that the metal components of combustion products, such as ROFA, may be involved in the increase in susceptibility to pulmonary infection as discussed in the sections above.

Immunomodulation by metals in the lungs can occur through alterations in primary (innate) defense mechanisms, such as physical barriers (nasal structures and hairs, epithelial barrier), mucociliary clearance, reflexes associated with innervation of 
epithelial cells, surfactant components, and phagocytes. Metals can also alter adaptive immunity in the lung, both humoral and cell-mediated. Not all metals are toxic and the toxicity of the inorganic form of the metal will depend on the chemistry, size, and solubility (ATSDR, 2005a; 2005b; 1999; Cohen, 2004). For the purpose of this review, the primary focus will be on the immunotoxic properties of $\mathrm{Al}, \mathrm{Fe}, \mathrm{Ni}, \mathrm{V}$, and $\mathrm{Zn}$, some of the primary metals associated with fossil fuel combustion. The insoluble fractions of the combustion products are generally composed of oxides whereas the soluble portions are primarily sulfates. Because much of the data regarding ROFA indicate that the soluble portion contributes significantly to the adverse effects of ROFA, more emphasis will be placed on the soluble forms of the metals, the chlorides and sulfates.

\section{Aluminum (Al)}

$\mathrm{Al}$ is one of the most abundant elements in the environment. However, inhalation by the general public is considered negligible, with the majority of exposures coming from occupations such as mining, smelting, welding, and production of $\mathrm{Al}$ powder (ATSDR, 1999). The majority of $\mathrm{Al}$ compounds are in the form of alumina $\left(\mathrm{Al}_{2} \mathrm{O}_{3}\right)$. Most information on lung disorders associated with $\mathrm{Al}$ compounds comes from occupational exposures, primarily from studies on potroom workers, where $\mathrm{Al}$ metal is recovered from ore (bauxite). These include increased incidence of pneumonia, asthma, bronchitis, sarcoid-like granulomas, pneumoconiosis, eosinophilia, and fibrosis (ATSDR, 1999; Cohen, 2004; Kelleher et al., 2000). However, the direct effects of Al compounds are unclear as these workers are also co-exposed to Fe, hydroxides, nitrates, fluorides, and other elements that affect the incidence of disease (ATSDR, 1999). Death due to respiratory exposure to extreme levels of $\mathrm{Al}$ is rare, and little or no systemic effects are 
reported after inhalation of $\mathrm{Al}$ (ATSDR, 1999). Al absorbance depends on solubility, and because the majority of exposures are to the insoluble forms of $\mathrm{Al}$, it tends to accumulate in the lung and toxicity appears to be primarily due to dust overload (ATSDR, 1999; Cohen, 2004). In fact, Berry et al. (1988) showed that even the soluble form of Al does not readily cross an epithelial barrier; therefore, $\mathrm{Al}$ absorption is usually low. Respiratory exposure to the oxide form of $\mathrm{Al}$ in animals has demonstrated slightly increased macrophage and neutrophil influx into the lungs (White et al., 1987) and caused shortlived increases in indices of lung injury (Lindenschmidt et al., 1990).

Animal studies have provided some information as to the effects of pulmonary exposure to the soluble forms of $\mathrm{Al}$, the chlorides and sulfates. It appears that only the soluble forms, particularly the sulfate form, have been shown to alter pulmonary host defense responses to bacterial infection (Drummond et al., 1986; Hatch et al., 1981); however, contradictory evidence to this also exists (Ehrlich, 1980). The soluble Al compounds have been found to alter function of cells involved in innate immunity. Drew et al. (1974) showed that Al chlorhydrate could induce macrophage and neutrophil influx and development of granulomatous foci at overload levels in hamsters. Finehi et al. (1981) found AM damage after long term exposure of rats to Al fluoride or Al chloride, and Castranova et al. (1980a) showed that rat AMs exposed to Al chloride in vitro had decreased production of superoxide anion, an antimicrobial agent. In terms of lymphocyte toxicity, very little information exits regarding effects of pulmonary exposure to $\mathrm{Al}$ on the function of $\mathrm{B}$ and $\mathrm{T}$ cells. However, $\mathrm{Al}$ compounds are widely used as adjuvants in vaccinations, and through this route of administration, $\mathrm{Al}$ is very effective at increasing the potency of the vaccination and stimulating both humoral and cell- 
mediated immune responses (Brewer et al., 1996; Lindblad, 2004). Whether or not Al has the ability to act in an adjuvant fashion after respiratory exposure in not well investigated.

\section{Iron (Fe)}

Fe is a crustal element that is found primarily in combination with other elements, mainly oxides, carbonates, sulfides, and silicates in a variety of mineral ores (Merrill et al., 2001). Fe is released into the atmosphere through both natural events, such as volcanic eruption, as well as from anthropologic sources, including production of Fe and steel, smelting, and through the combustion of fossil fuels (Brooks, 1981; Jacob and Gottlieb, 1989; Munger et al., 1983). The most common valence states of Fe are 2+ (ferrous) and 3+ (ferric), and Fe can exist in up to 16 different oxide forms depending on conditions during formation, with the most common encountered being that of $\mathrm{Fe}_{2} \mathrm{O}_{3}$ (Ball et al., 2000). The most soluble forms of Fe are the salts in the forms of sulfates and chlorides, as well as ferric nitrate (NIOSH, 2005). The primary route of exposure in humans is oral, and Fe is an essential element in the diet, where a deficiency or overload can compromise the immune system and result in a number of different illnesses (Omara et al., 1998). Although respiratory exposure can occur in the general public due to atmospheric emissions, much higher pulmonary exposures occur in a variety occupations, including, but not limited to, foundry works, smelting, mining and refining, steel grinding, welding, silver and steel polishing, stone cutting, and boiler cleaning (Brooks, 1981). However, it should be noted that occupational exposure to Fe is usually concurrent with other more toxic substances, such as silica, quartz, PAH, asbestos, and other metals (Brooks, 1981; Castranova et al.1997; Finkelman, 1995; Garcon et al., 2004; Hardy and 
Aust, 1995; Meyers, 1982; Nemery, 1990). Therefore, effects specific to Fe inhalation on pulmonary susceptibility to infection are less clear.

Occupations associated with iron exposure have also been correlated with increases in infection, primarily tuberculosis (Starzynksi et al., 1996); however, concomitant exposure to a wide variety of other compounds has made it difficult to implicate $\mathrm{Fe}$ as the primary agonist. Occupational exposure to $\mathrm{Fe}_{2} \mathrm{O}_{3}$ over time can lead to a form of pneumoconiosis referred to as siderosis (Brooks, 1981), which is defined as a benign, and relatively non-symptomatic, form of pneumoconiosis. In historical terms, inhalation of $\mathrm{Fe}_{2} \mathrm{O}_{3}$ in humans has not been shown to be toxic, allergic, pathogenic (Brooks, 1981; Kleinerman et al., 1974; Kleinfeld et al., 1969; Lay et al., 2001), or carcinogenic (Steinhoff et al., 1991; Stokinger, 1984), and in animals studies, $\mathrm{Fe}_{2} \mathrm{O}_{3}$ has been used as a control dust for particle exposure and is considered to be relatively inert (Beck et al., 1982; Gibb and Morrow, 1962; Morrow et al., 1964; Wright et al., 1989). AMs have been shown to be activated by $\mathrm{Fe}_{2} \mathrm{O}_{3}$ to clear the particles from the lungs (Lehnert and Morrow, 1985), and the effects of $\mathrm{Fe}_{2} \mathrm{O}_{3}$ on infection in animal models have mixed results. Aranyi et al. (1983) found increased mortality in mice after exposure to $\mathrm{Fe}_{2} \mathrm{O}_{3}$. However, other studies examining the effects of $\mathrm{Fe}_{2} \mathrm{O}_{3}$ on infectivity alone, or as a major component of a relatively insoluble form of welding fume, indicate that respiratory exposure to the particulate form does not alter bacterial clearance or mortality in rodents (Antonini et al., 2004b; Hatch et al., 1981).

More recently, animal studies have shown that particulate Fe may not be as benign as once thought. Warheit et al. (1997) have shown that if the concentration of a low toxicity dust is high enough, it can impair clearance mechanisms and increase 
inflammation in rodents. Other studies have shown that Fe in particles has the ability to participate in a Fenton-like reaction and contribute to the generation of reactive oxygen species, and oxidative stress (Ghio et al., 1998; Lucesoli et al., 1999; Simeonova and Luster, 1995). Studies have also shown that AMs can sequester Fe and possibly protect nearby cells from Fe-catalyzed oxidants (Olakanmi et al., 1993). Other researchers indicate that the oxidant production in response to Fe exposure is due to the small amount of soluble Fe associated with the particulates (Lay et al., 1999), the ability of the cells to mobilize free Fe from the particulate (Ball et al., 2000; Smith et al., 1998; Watson and Brain, 1979), or the interactions with other particles that may cause Fe to become bioavailable (Pinkerton et al., 2004; Zhou et al., 2003).

There is more evidence for adverse effects of Fe on the lungs in its soluble, or bioavailable, form. In terms of alterations in susceptibility to infection, Hatch et al. (1981) have shown that, although respiratory exposure to $\mathrm{Fe}_{2} \mathrm{O}_{3}$ in mice did not cause any significant mortality after bacterial infection, there were intermediate increases in mortality when mice were exposed to Fe sulfates, particularly $\mathrm{FeNH}_{4}\left(\mathrm{SO}_{4}\right)_{2}$, prior to infection. In addition, Sherwood et al. (1981) found that inhalation of $\mathrm{FeSO}_{4}$ increased susceptibility to Staphylococcus aureus, but the bacterial dose had to be sufficient in order to observe this effect, as other researchers did not achieve this response with a lower dose (Goldstein et al., 1979). Zelikoff et al. (2002) demonstrated that exposure to $\mathrm{FeCl}_{2}$ could alter innate and adaptive immune parameters in uninfected rats, and that exposure to soluble $\mathrm{Fe}$ after infection altered the clearance of bacteria from the lungs of rats. The mechanisms by which the soluble forms of Fe may cause alterations in infectivity are not well understood. Much of the recent data on the effects of soluble Fe 
on various cells in the lungs come from studies that have examined the responses to soluble Fe as it exists in PM.

As mentioned previously, combustion particles have significant levels of metals, and adverse effects, in vivo and in vitro, have been associated with the soluble portions of these samples. Veranth et al. (2000) have demonstrated that Fe can be better mobilized from combustion sources (coal and oil) than noncombustion sources (mineral dust). Huang et al. (2005) have correlated the levels of bioavailable Fe in coal with increased incidences of coal workers' pneumoconiosis (CWP). Also, bioavailable Fe has been implicated in increased transcription factor activation, which may lead to increased inflammation, cytokine production and oxidative stress (Huang et al., 2002b; Zhang and Huang, 2003). Soluble Fe in CFA has also been associated with increased production of cytokines and reactive oxygen species in epithelial cells (Ball et al., 2000).

As discussed previously, $\mathrm{FeSO}_{4}$, in the concentrations present in a ROFA sample, has been shown to induce lung injury, increase lung permeability, enhance inflammation, and alter cytokine production in rodents (Kodavanti et al., 1997; 1998; Nadadur and Kodavanti, 2002), and soluble Fe in ROFA may induce eosinophil influx and possibly alter airway hypersensitivity in rats (Lambert et al., 2000). However, unlike CFA, the role for oxidant production in ROFA has been more closely correlated with vanadyl sulfate $\left(\mathrm{VOSO}_{4}\right)$ than with $\mathrm{FeSO}_{4}$ (Ghio et al., 2002; Huang et al., 2003; Kadiiska et al., 1997). This may be due to the form or concentration of the bioavailble Fe in these two combustion ashes.

It is clear that soluble Fe has adverse effects on AMs, and alterations in AM activity may ultimately affect innate immune responses in the lung. Inhalation of $\mathrm{FeCl}_{3}$ in 
rabbits has been shown to increase AM influx into the lung, increase AM lysosome and phospholipid content, and enhance the phagocytic capacity of AMs (Johansson et al., 1992). Exposure of AMs in vitro to Fe chlorides has resulted in increased reactive oxygen species production (Castranova et al., 1980a; Schluter et al., 1995) and induced hyperoxic injury to AMs (Wesselius et al., 1999). After inhalation of $\mathrm{Fe}_{2}\left(\mathrm{SO}_{4}\right)_{3}$ in hamsters, AMs were found to have decreased phagocytic rates (Skornik and Brian, 1983).

Fe deficiency and overload as result of diet has been shown to have a host of adverse effects on lymphocytes, as well as innate immune cells (Omara et al., 1998); however, there are very few studies that have examined the effects on cells involved in adaptive immune responses after respiratory exposure to soluble forms of Fe. Zelikoff et al. (2002) did show that inhalation of soluble Fe, in the absence of infection, resulted in decreased lymphocyte values in the blood, increased superoxide anion production by monocytes, and reduced proliferation of B lymphocytes stimulated with lipoplysaccharide (LPS). In addition, soluble Fe altered unstimulated, but not stimulated, $\mathrm{T}$ cell proliferation. Furthermore, inhalation of soluble Fe after pulmonary bacterial infection led to increased numbers of bacteria in the lung. Based on the bioavailability of $\mathrm{Fe}$, the source or form of soluble $\mathrm{Fe}$, and the dose, both innate and adaptive pulmonary immune responses may be altered after respiratory exposure.

\section{Nickel (Ni)}

$\mathrm{Ni}$ is classified as a hard metal and occurs naturally in soil and volcanic dust. It can be released into the atmosphere as a windblown dust or in volcanic ash; however, the majority of $\mathrm{Ni}$ is released into the atmosphere from anthropologic sources, including fossil fuel combustion, waste incineration, alloy production, smelting, mining and 
refining (ATSDR, 2005a; Cohen, 2004; Smialowicz, 1998). The form of nickel in the air is dependent on the source, and $\mathrm{Ni}$ from anthropologic sources include complex $\mathrm{Ni}$ oxides (NiO), Ni sulfate $\left(\mathrm{NiSO}_{4}\right)$, and metallic $\mathrm{Ni}(\mathrm{ATSDR}, 2005 \mathrm{a})$. The relatively insoluble forms of $\mathrm{Ni}$ include $\mathrm{Ni}$ subsulfide $\left(\mathrm{Ni}_{3} \mathrm{~S}_{2}\right)$ and $\mathrm{NiO}$, although these compounds will have some degree of solubility, and solubility of oxides also depends on the source (ATSDR, 2005a; Kelleher et al., 2000; Takahashi et al., 1992; Yamada et al., 1993). Ni exposure in the general public is primarily from food and water. Occupational exposure can occur through a variety of exposure routes including inhalation and dermal contact, and the lungs and immune system are the primary targets after inhalation of $\mathrm{Ni}$ compounds (ATSDR, 2005a). The most common adverse health effect associated with Ni in general is allergic contact dermatitis via dermal exposure, where approximately 10$20 \%$ of the general population is sensitized to Ni (ATSDR, 2005a). The International Agency for Research on Cancer (IARC) has classified metallic Ni as possibly carcinogenic to humans and $\mathrm{NiO}$ and $\mathrm{Ni}_{3} \mathrm{~S}_{2}$ as carcinogenic to humans, and the EPA has classified $\mathrm{Ni}$ refinery dust and $\mathrm{Ni}_{3} \mathrm{~S}_{2}$ as carcinogenic to humans, resulting in nasal and lung cancer after inhalation (ATSDR, 2005a). Soluble Ni compounds do not appear to be overtly carcinogenic (ATSDR, 2005a; Kasprzak et al., 2003; Oller, 2002; Oller et al., 1997); however, lung toxicity (inflammation and injury) and impaired immune responses appear to be a function of solubility (NTP, 1996a; 1996b; 1996c) with the order of toxicity as $\mathrm{NiSO}_{4}>\mathrm{NiS}>\mathrm{NiO}$. In $\mathrm{Ni}$ welders and foundry workers, inhalation exposure has been shown to result in a range of diseases including chronic bronchitis, emphysema, pulmonary fibrosis, asthma, chemical pneumonitis, and cancer (ATSDR, 2005a; Hovey et al., 1983; Kelleher et al., 2000; Snow and Costa, 1998). 
Inhalation studies in rodents have shown increased pulmonary inflammation, epithelial cell and macrophage hyperplasia, lymph node hyperplasia, impaired immune function (Benson et al., 1988; 1995; Dunnick et al., 1989; Haley et al., 1990; Hirano et al., 1994; NTP, 1996a; 1996b; 1996c) and, at higher doses, there is evidence of fibrosis (Dunnick et al., 1989). The general consensus from all these studies was that the soluble forms of $\mathrm{Ni}$ were more toxic than the insoluble forms, and that the immune system was a target for soluble Ni after inhalation exposure. In addition, acute lung injury and cytotoxicity have been associated with $\mathrm{NiSO}_{4}$ at concentrations present in ROFA, as discussed above (Dreher et al., 1997; Kodavanti et al., 1998). The study by Sunderman et al. (1989) which subjected rats to $\mathrm{Ni}$ chloride $\left(\mathrm{NiCl}_{2}\right)$ via intraperitoneal injection, demonstrated that the lungs, and AMs in particular, are also a target for Ni that enters the system through a non-respiratory route. Knight et al. (1991) also found that intraperitoneal injection of $\mathrm{NiSO}_{4}$ had adverse effects on the lung, thymus, and spleen. In animal models, both respiratory exposure to and intraperitoneal injection of $\mathrm{Ni}$ has been shown to enhance susceptibility to respiratory infection (Adkins et al., 1979; Daniels et al., 1987; Ehrlich, 1980; Port et al., 1975; Zelikoff et al., 2002). In terms of effects on innate immune cells, numerous studies have been performed in vivo and in vitro to assess effects of Ni on AMs. Some effects on macrophage function in response to $\mathrm{Ni}$ in combustion-derived particles have been discussed in a previous section. In addition to those studies, suppression of AMs is well documented, particularly in response to the soluble forms of Ni (Aranyi et al., 1979; Camner et al., 1984; Castranova et al., 1980b; Graham et al., 1975; Goutet et al., 2000; Haley et al., 1990; Johansson and Camner, 1980; Riley et al., 2005; Schluter et al., 1995; Sunderman et al., 1989). In these studies 
phagocytosis appeared to be inhibited, cytotoxicty was increased, metabolic effects varied but production of oxidants appeared to be inhibited, and lipid peroxidation was elevated.

NK cells also appear to be a target for Ni toxicity, though the majority of data concerning their activity comes from in vitro studies and studies with intraperitoneal injection as the major route of exposure (Smialowicz, 1998). Goutet et al. (2000) showed that respiratory exposure to soluble Ni caused NK cell cytotoxicity. However, Daniels et al. (1987) found that only parenteral exposure, not inhalation, resulted in decreased NK cell activity. Although respiratory exposure data are inconsistent, parenteral exposure and in vitro data tend to indicate that soluble Ni may have adverse effects on NK cells (Condevaux et al., 2001; Smialowicz, 1998; Smialowicz et al., 1985; 1987; Zeromski et al., 1995). In addition to the effects on AM and NK cell function, DCs also appeared to be affected by Ni (ATSDR, 1995a). The majority of studies concerning DCs refer to Langerhans cells in the skin in relation to contact dermatitis. An in vitro study by De Smedt et al. (2001) showed that $\mathrm{NiCl}_{2}$ altered DC surface markers and increased production of interleukin (IL)-6, tumor necrosis factor (TNF)- $\alpha$, and IL-12, proinflammatory cytokines that also play roles in directing adaptive immune responses. The variety of effects on these cells that are involved in the innate immune system may ultimately influence the increase in infectivity observed in response to $\mathrm{Ni}$ compounds.

In addition to effects on innate immune function, $\mathrm{Ni}$ compounds also have the ability to alter the adaptive immune response; however, the majority of the data regarding lymphocyte function come from in vitro studies, and routes of exposure other than inhalation (ATSDR, 2005a; Cohen, 2004). Benko et al. (1983; 1986) conducted studies 
on workers exposed to Ni compounds and found that $\operatorname{IgA}$ and $\operatorname{IgM}$ were increased and IgE levels were decreased, indicating $\mathrm{Ni}$ has effects on B cell function. In rodent studies, parenteral exposure to $\mathrm{NiCl}_{2}$ and $\mathrm{NiSO}_{4}$ suppressed $\mathrm{T}$ lymphocyte proliferation and cytokine production, and contributed to the degeneration of thymocytes and spleenocyte in vivo, respectively (Harkin et al., 2003; Knight et al., 1991; Li et al., 1988; Smialowicz et al., 1984). In vitro, $\mathrm{NiCl}_{2}$ has been shown to induce apoptosis in $\mathrm{T}$ cells (Kim et al., 2002) and cause DNA damage in human lymphocytes (Chen et al., 2003).

Taken together, soluble Ni compounds have been shown to alter many aspects of the immune system through a variety of exposure routes. The studies highlighted above show that both innate and adaptive immune responses may be adversely affected by the soluble forms of Ni. Therefore, not only suppressive effects on innate immune responders, such as macrophages, but also alterations in B and T cell activity, as well as targeting of the lung after exposure to $\mathrm{Ni}$, may contribute to the increased susceptibility to pulmonary infection that has been observed by several investigators (Adkins et al., 1979; Daniels et al., 1987; Ehrlich, 1980; Ehrlich et al., 1978; Port et al., 1975; Zelikoff et al., 2002).

\section{Vanadium (V)}

$\mathrm{V}$ is a ubiquitous trace element, found in at least 50 different mineral ores, and occurs naturally in fossil fuels and oils (ATSDR, 1992; Cohen, 2004). V can exist in 6 different oxidation states, the most common being $\mathrm{V}^{3+}, \mathrm{V}^{4+}$, and $\mathrm{V}^{5+}$, and is usually associated with oxygen. Oral exposure from the diet is the primary route of exposure in the general public; however, in areas where oil is burned for energy there can be significant exposure via inhalation (ATSDR, 1992; Zoller et al., 1973), more so in winter 
months as energy consumption increases (Lee et al., 1972). Burning of fossil fuels contributes to the majority of airborne $\mathrm{V}$, primarily in the form of vanadium pentoxide $\left(\mathrm{V}_{2} \mathrm{O}_{5}\right)$, which is highly insoluble, and the most prevalent soluble form is vanadyl sulfate $\left(\mathrm{VOSO}_{4}\right)(\mathrm{ATSDR}, 1992)$. Occupational exposure can occur through multiple routes including inhalation which results primarily from mining and milling $\mathrm{V}$ in ores, oil-fired boiler cleaning, and production of $\mathrm{V}$ as a metal (as in the steel industry), an oxide, or a catalyst (ATSDR, 1992; Cohen, 2004). Once inhaled, approximately $40 \%$ of the soluble or insoluble $\mathrm{V}$ can be cleared from the lungs within 1 hour; however, after 24 hours the insoluble form is much more persistent (Conklin et al., 1982; Edel and Sabbioni, 1988; Sharma et al., 1987). V can also locate to the lung after non-inhalation routes of exposure as well (ATSDR, 1992; Cohen, 2004; Hopkins and Tilton, 1966, Kacew et al., 1982).

The majority of information regarding effects on respiratory immune function center around pentavalent $\mathrm{V}$ in its insoluble form, and alterations in pulmonary immunity are known to be associated with the insoluble vanadates and oxides. Worker exposures to airborne vanadates had an increased incidence of tuberculosis, respiratory tract irritation, and bacterial-induced respiratory failure (Cohen, 2004; Dutton, 1911). Other pulmonary problems associated with occupational exposure to insoluble $\mathrm{V}$ compounds include asthma, rhinitis, pharyngitis, chronic bronchitis ("Boilermakers Bronchitis"), and pneumonia (Cohen, 2004; Irsigler et al., 1999; Lees, 1980; Levy et al., 1984; Musk and Tees, 1982; Sjorberg, 1955; Woodin et al., 2000). V can also produce a fume-like fever (Vandenplas et al., 2002), and increased fibrotic loci (Kivuoloto et al., 1979). The association of $\mathrm{V}$ with cancer in humans is less clear due to other confounding components in exposure; however, a long-term animal study by the National Toxicology 
Program (NTP) (2002) indicated that chronic exposure may result in increased incidences of cancer.

Animal studies are in agreement with most of the epidemiological data. Effects of pentavalent $\mathrm{V}$ in animal and cellular models include, but are not limited to, decreased macrophage phagocytosis and enzyme activity, altered immune cell populations and activity (particularly macrophages, neutrophils, and T cells), altered cytokine production, decreased bactericidal and tumoricidal factors, disruption of expression of major histocompatibility complex (MHC), increased chemotactic factors, increased cytotoxicity in the lung, and enhanced oxidative damage to pulmonary cells (ATSDR, 1992; Bonner et al., 2000; Castranova et al., 1984; Cohen, 2004; Chien et al., 2006; Chong et al., 2000; DiGioacchino et al., 2002; Ehrling et al., 2000; Fisher et al., 1986; Kleinsasser et al., 2003; Ress et al., 2003; Saldiva et al., 2002; Shen et al., 2001; Toya et al., 2001;Wang et al., 2003).

Pulmonary effects of the more soluble forms of $\mathrm{V}, \mathrm{VOSO}_{4}$ and $\mathrm{VCl}_{2}$, come primarily from studies that focus on fly ash, and ROFA in particular. Many of these studies have been reviewed in a previous section. Briefly, the effects included increased lung injury, cytotoxicity, changes in macrophage function and oxidative potential, alterations in cell signaling, and altered cytokine production. Hatch et al. $(1981 ; 1985)$ employed an infectivity study in mice to determine the effect of various metals on the ability of the rodents to recover from a bacterial infection. The authors found that $\mathrm{VOSO}_{4}$ did slightly increase the severity of the infection although only modestly as compared to other metals. Riley et al. (2005) showed that AMs were more sensitive to $\mathrm{VCl}_{2}$ than pulmonary epithelial cells were in terms of cytotoxicity; however, macrophages appeared 
to be more sensitive to $\mathrm{Zn}$ and $\mathrm{Cu}$ chlorides in general. It appears that many of the effects of $\mathrm{V}$, the soluble and insoluble forms, can be attributed to its ability to participate in redox reactions and to produce free radicals (Ghio et al., 2002; Huang et al., 2003;

Kadiiska et al., 1997; Schluter et al., 1995; Sorensen et al., 2005; Wang et al., 2003). Another aspect of $\mathrm{V}$ is its ability to increase production of interferon (IFN)- $\gamma$ by lymphocytes (Shen et al., 2001) and to enhance cellular responses to interferon (Cohen et al., 1996, 1997), which can have downstream effects on calcium signaling, production of reactive oxygen species, cytokine responses, and expression of MHC (Cohen, 2004). These responses are important in reactions to pulmonary infections and may ultimately have an impact on innate and cell-mediated immunity in the lung.

\section{Zinc (Zn)}

$\mathrm{Zn}$ is an abundant trace metal in the environment, existing primarily as $\mathrm{Zn}$ oxide $(\mathrm{ZnO})$ and sphalerite $(\mathrm{ZnS})$, and is released into the environment via mining, smelting, steel production, burning of wastes, and combustion of fossil fuel. As with the majority of the metals discussed, ingestion of $\mathrm{Zn}$ is the primary route of exposure in the general population (ATSDR, 2005b). Zn is an essential dietary element; however, dietary deficiency or overload can result in a number of adverse effects including impaired immune function (ATSDR, 2005b; Cohen, 2004). Adverse effects are also associated with inhalation of $\mathrm{Zn}$. Although ambient air levels of $\mathrm{Zn}$ are higher in industrial areas compared to more rural regions, the majority of individuals exposed to higher concentrations of $\mathrm{Zn}$ are tobacco smokers and workers involved in galvanizing, smelting, welding, or brass foundry operations (ATSDR, 2005b; Cohen, 2004). 
Metal fume fever is the disease most commonly associated with inhalation of $\mathrm{ZnO}$ and has also been reported after exposure to other metal oxide including $\mathrm{Cd}, \mathrm{Cu}, \mathrm{Cr}, \mathrm{Mg}$, Ni and titanium (Ti) (ATSDR, 2005b; Blanc et al., 1991; Cohen, 2004; Kelleher et al., 2000). Fume fever is characterized by dyspnea, cough, fever, and malaise. Onset occurs within hours after inhalation; however, disease does not progress and resolves within a few days (Drinker et al., 1927; Lehman et al., 1910). Increases in neutrophils, leukocytes, T cells, NK cells, LDH, and pro-inflammatory cytokines occur in the lungs in association with $\mathrm{ZnO}$ exposure (Blanc et al., 1991; Conner et al., 1988; Gordon et al., 1992;

Kuschner et al., 1997), and $\mathrm{ZnO}$ has been shown to inhibit macrophage phagocytosis (Fisher et al., 1986). In addition, the oxide form of $\mathrm{Zn}$ has also been associated with increased susceptibility to pulmonary bacterial infection in mice (Hatch et al., 1985). Zinc oxide has been widely studied; however, less in known regarding the soluble forms of $\mathrm{Zn}$ encountered in industrial and environmental settings (Cohen, 2004).

A case study has shown pneumonitis after exposure to a very excessive amount of Zn chloride $\left(\mathrm{ZnCl}_{2}\right)\left(1,955 \mathrm{mg}\right.$ zinc/m $\left.{ }^{3}\right)$ (Johnson and Stonehill, 1961), and another study showed pneumonitis in a smelter worker in relation to Zn exposure (Ameille et al., 1992). Long-term animal studies on guinea pigs have found development of alveolitis and fibrosis in response to $\mathrm{ZnCl}_{2}$ (Marrs et al., 1988). In a rodent model, $\mathrm{ZnSO}_{4}$ has been shown to enhance susceptibility to pulmonary bacterial infection (Ehrlich, 1980; Hatch et al., 1985); however, there is limited information regarding the effects of the soluble forms of $\mathrm{Zn}$ on immune cells in the lung after respiratory exposure. In terms of cellular responses, the majority of data regarding respiratory exposure to soluble $\mathrm{Zn}$ focuses on inflammation and macrophage responses. In rodents, $\mathrm{ZnCl}_{2}$ can lead to increased 
infiltration of macrophages and lymphocytes (Marrs et al., 1988; Prieditis and Adamson, 2002). Riley et al. (2005) have shown that macrophages are more sensitive to $\mathrm{ZnSO}_{4}$ than epithelial cells, and like the chloride form, $\mathrm{ZnSO}_{4}$ associated with combustion emissions can induce neutrophil influx and increase indicators of lung damage after exposure in rats (Kodavanti et al., 2002). Skornik and Brain (1983) found that $\mathrm{ZnSO}_{4}$, like $\mathrm{ZnO}$, could inhibit AM phagocytosis after respiratory exposure in hamsters. Therefore, there is a small amount of information on the effects of innate immune responders in the lungs (macrophages and neutrophils); however, there is very little research in the area of adaptive pulmonary immune responses or how soluble $\mathrm{Zn}$ as it exists in combustion particles may alter susceptibility to respiratory infection.

\section{Listeria monocytogenes Infection}

Listeria monocytogenes is a gram-positive, intracellular (cytosolic), facultative bacteria. Infection with the bacteria is of concern primarily to immunocompromised individuals (individuals that have an increased susceptibility to infection), as well as neonates and pregnant women. It has been used as a model to study immune responses to infection primarily because the basic progression of the infection is predictable, the bacteria is easy to culture, and it is relatively safe to handle (Pamer, 2004). Although the

primary route of entry for L. monocytogenes is through ingestion, most animal study models use intraperitoneal or intravenous injection. In addition, respiratory models of the listeriosis paradigm have been used by a number of investigators to characterize lung immune responses and to determine the effects of various environmental exposures on these responses (Antonini et al., 2002; Cohen et al., 2001; Jakab, 1993; MacLean et al., 
1996; Reasor et al., 1996; Steerenberg et al., 2004; van Loveren et al., 1988; Yin et al., 2004a).

Both the innate and adaptive immune responses are required to clear $L$.

monocytogenes infection. The innate immune response is the first line of defense against infection and is characterized by nonspecific responders such as monocytes, macrophages, neutrophils, NK cells, and DCs. The adaptive immune response is classified as naïve, must be developed and is, therefore, delayed but highly specific. Adaptive immunity can be divided into two divisions, the humoral response, governed by B cells and aided by CD4+ $\mathrm{T}_{\mathrm{H}} 2$ cells, and the cell-mediated response, where CD8+ T cells and $\mathrm{CD} 4+\mathrm{T}_{\mathrm{H}} 1$ are the primary responders. The adaptive immune response to $L$. monocytogenes is dominated by cell-mediated immunity (McGregor et al., 1970; Pamer, 2004) with important roles for both CD8+ T cells and CD4+ $\mathrm{T}_{\mathrm{H}} 1$ cells (Seder and Gazzinelli, 1999; Hsieh et al., 1993).

Inability of an organism to elicit an innate immune response to L. monocytogenes infection is lethal (Unanue, 1997). Phagocytosis of the bacteria by the macrophage is one of the early critical processes that will lead to a series of events dictating the immune response to the infection. One of the outcomes of phagocytosis is the initiation of a proinflammatory cytokine cascade that includes the production of TNF- $\alpha$, IL-1, IL-6, and IL-12 (Seder and Gazzinelli, 1999). The inflammatory response, in turn, will result in an increase in endothelial barrier permeability, an increase in neutrophil and monocyte recruitment to the infected area, and enhanced activation of phagocytes and NK cells in order to kill the bacteria. 
Deficiencies in both TNF- $\alpha$ (Rothe et al., 1994) and IL-6 (Dalrymple et al., 1995; Kopf et al., 1994) result in a decrease in neutrophil recruitment and a lethal $L$. monocytogenes infection in mice. Neutralization of IL-1 in mice results in enhanced susceptibility to Listeria infection (Havell et al., 1992; Rogers et al., 1994). It has been suggested that the increase in susceptibility due to the lack of, or the decrease in, these cytokines may be related to their role as co-stimulators, along with IL-12, of NK cell production of IFN- $\gamma$ (Hunter et al., 1995; Wherry et al, 1991). IFN- $\gamma$ is an activator of both macrophages and NK cells and enhances killing activity by these cells. It has been shown that mice that are deficient in IFN- $\gamma$ do not survive infection with $L$. monocytogenes (Buchmeier et al., 1985).

Early resistance to L. monocytogenes infection is dependent on the production of IFN- $\gamma$ by NK cells, and this, in turn, is highly dependent on the presence of IL-12 (Tripp et al., 1993; 1994) and IL-18 (Berg et al., 2002; Neighbors et al., 2001), both of which are produced by DCs and macrophages. IL-12 and IL-18 are also factors in driving the differentiation of immature $C D 4+T$ cells $\left(\mathrm{T}_{\mathrm{H}} 0\right)$ toward the $\mathrm{T}_{\mathrm{H}} 1$ subset, another producer of IFN- $\gamma$ (Hsieh et al., 1993; Stoll et al., 1998). The critical role for IFN- $\gamma$ is to further activate macrophages by enhancing their ability as antigen presenting cells (APCs) and inducing production of reactive species such as $\mathrm{NO}$ (a mediator of intracellular killing of the bacteria) (Seder and Gazzinelli, 1999). Mice that are deficient in inducible NO synthase (iNOS) (MacMicking et al., 1995) and phagocyte oxidase (Shiloh et al., 1999) produce less reactive nitrogen and oxygen species and are more susceptible to infection. However, endogenous NO is also an inhibitor of the cell-mediated response, suppressing proliferation of $\mathrm{CD} 4+$ and, to a greater extent, CD8+ T cells at high levels (Blesson et al., 
2002; Hoffman et al., 2002; van der Veen et al., 1999; 2000). Therefore, the correct balance of $\mathrm{NO}$ is necessary in regulating the immune response. In addition to direct stimulatory effects on cells, IFN- $\gamma$ also decreases the production of IL-10 (an antiinflammatory cytokine that promotes the humoral immune response) by macrophages and enhances IL-12 production by APCs (macrophages and DCs). This process favors the development of $\mathrm{T}_{\mathrm{H}} 1$ as opposed to $\mathrm{T}_{\mathrm{H}} 2 \mathrm{CD} 4+\mathrm{T}$ cells. Therefore, IL-12, in addition to its role in the innate response to the bacteria, is crucial to the adaptive immune response by directing $\mathrm{T}$ cell differentiation toward cell-mediated immunity, rather than humoral immunity.

Pamer, 2004, has reviewed numerous studies that examined the importance of the adaptive immune response to $L$. monocytogenes. These studies show that mice that lack cell-mediated and humoral immunity are resistant to the infection at early time points, but cannot effectively clear the bacteria over time (Bancroft et al., 1991; Emmerling et al., 1975; Nickol and Bonventre, 1977). In addition, a study by Ladel et al. (1994) showed the importance of both the MHC I-dependent CD8+ T cell and the MHC II-dependent CD4+ T cells response to $L$. monocytogenes.

The MHC II-dependent CD4+ T cells responses to L. monocytogenes result primarily in the development of the $\mathrm{T}_{\mathrm{H}} 1$ subset of CD4+ T cells. APCs, such as DCs and macrophages, which ingest pathogens, will present antigens from the bacteria in conjunction with $\mathrm{MHC}$ II on the cells surface to $\mathrm{T}_{\mathrm{H}} 0 \mathrm{CD} 4+\mathrm{T}$ cells in the lymphoid tissues. Although the mechanisms that control the differentiation of these $\mathrm{T}$ cells are not completely understood, there is a clear role for cytokines during $\mathrm{T}$ cell activation (Janeway et al., 2001). IL-10 and IL-4 will favor promotion of $\mathrm{T}_{\mathrm{H}} 2$ cells and inhibit that 
of the $\mathrm{T}_{\mathrm{H}} 1$ subset (Mosmann and Sad, 1996). The presence of IL-12, IL-18, and IFN- $\gamma$, and the absence of IL-10, is critical in promoting the differentiation of CD4+ T cells toward the $\mathrm{T}_{\mathrm{H}} 1$ subset as part of the cell-mediated immune response, which is the dominant response to L. monocytogenes. Activated $\mathrm{T}_{\mathrm{H}} 1$ cells will produce cytokines, such as TNF- $\alpha$ and IFN- $\gamma$, which will aid in the activation of macrophages and NK cells in killing intracellular bacteria, and IL-2, a cytokine that promotes the growth and proliferation, or clonal expansion, of $\mathrm{T}$ cells involved in the response.

L. monocytogenes can infect almost any cell type (Seder and Gazzinelli, 1999). Therefore, macrophages can also become infected with L. monocytogenes after phagocytosis, whereby the bacteria escape from the phagosome and enter the cytosol by producing a membrane pore-forming toxin, listeriolysin O (LLO) (Portney et al., 1988). Because L. monocytogenes is an intracellular bacteria that resides in the cytosol, it will be processed there and antigens from that bacteria will be expressed on the cell surface in conjunction with MCH I. CD8+ T cells, which recognize antigen with MHC I, are activated primarily by DCs, which in turn are aided by $\mathrm{CD} 4+\mathrm{T}$ cells that can increase the DCs stimulatory activity. Proliferation and differentiation of CD8+ T cells is driven by IL-2, which is produced by activated CD8+ T cells, acting in an autocrine fashion, and by $\mathrm{T}_{\mathrm{H}} 1$ cells. Activated CD8+ T cells can then produce cytokines, such as TNF- $\alpha$ and IFN- $\gamma$ discussed above, and cytotoxic effector molecules. CD8+ T cell recognition and interaction with antigen presented with MHC I will induce infected cells to enter the apoptotic cycle resulting in programmed cell death (Janeway et al., 2001).

To summarize, killing and clearing L. monocytogenes from the lung requires activation of AMs, neutrophils, and NK cells in the innate immune system, and primarily 
$\mathrm{CD} 4+\mathrm{T}_{\mathrm{H}} 1$ and $\mathrm{CD} 8+\mathrm{T}$ cells in the adaptive immune system. The two systems are intricately tied to each other and cytokine production appears to be an important form of communication between the two. In addition, the feedback from the adaptive immune responders signals macrophages and NK cells to become more effective at killing infected cells and clearing infection.

ROFA and the soluble component of ROFA, in absence of infection, have been shown to alter cellular responses that may be important in resolving an infection, as discussed above, including cytokine production, gene activation, activity of macrophages, and oxidative stress levels. Therefore, it is plausible that the soluble metals in ROFA may be responsible for altered susceptibility to pulmonary infection with L. monocytogenes; however, the mechanisms by which this may occur and the effects of ROFA on T cellmediated immune responses have not been fully investigated.

\section{$\underline{\text { Hypothesis and Specific Aims }}$}

The components in ROFA and the mechanism by which ROFA may alter immune responses to lung infection are not well characterized. ROFA has been shown to increase susceptibility to infection (Antonini et al., 2002; Hatch et al., 1985), but the components that are involved in the response have not been characterized. In addition, ROFA is known to alter the function of phagocytes in the absence of infection; therefore, it would be likely that the innate immune response would be altered by ROFA. However, little is known about its effects on other cells involved in innate immunity, including NK cells, and there have been very few studies that examine the mechanism by which ROFA may alter innate immune response to enhance susceptibility to infection. The direct effects of 
ROFA on lymphocytes, both B and T cells, are also not well characterized. Most of the information regarding the function of these cell types in response to ROFA comes from studies that examine the ability of ROFA to enhance sensitization and hyperresponsiveness to allergens in animals (Gavett et al., 1999; Lambert et al., 2000).

The hypothesis of this study was that soluble transition metals in ROFA may suppress innate and adaptive immune response to respiratory infection with a bacterial pathogen. The specific aims addressed in the following studies were: 1- demonstrate that the soluble fraction of ROFA increases susceptibility to infection; 2- determine potential mechanisms by which the soluble metals suppress the innate and adaptive pulmonary immune response to infection; and 3-identify the metal or metal combinations that are responsible for the alterations in pulmonary host defense.

To investigate this, an in vivo infectivity model was employed where rats were intratracheally instilled with ROFA, soluble or insoluble ROFA, or the individual soluble metals or metal combinations in ROFA on day 0 . On day 3 , rats were intratracheally inoculated with L. monocytogenes as the model pathogen. Pulmonary clearance, morbidity, cellular profiles and phenotypes, lung injury parameters, and cytokine and oxidant production were monitored on day 3 prior to infection, and for 1 week after infection. 
STUDY 1: SOLUBLE METALS ASSOCIATED WITH RESIDUAL OIL FLY ASH INCREASE MORBIDITY AND LUNG INJURY AFTER BACTERIAL

INFECTION IN RATS 


\section{$\underline{\text { Abstract }}$}

Inhalation of residual oil fly ash (ROFA) has been shown to impair lung defense mechanisms in laboratory animals and susceptible populations. Bioavailability of soluble transition metals has been shown to play a key role in lung injury caused by ROFA exposure. The goal of this study was to evaluate the effect of soluble metals on lung defense and injury in animals pre-exposed to ROFA followed by pulmonary challenge with a bacterial pathogen. ROFA was suspended in phosphate-buffered saline (R-Total), incubated overnight at $37^{\circ} \mathrm{C}$, and separated by centrifugation into soluble (R-Soluble) and insoluble (R-Insoluble) fractions. A portion of the soluble sample was treated with the metal-binding resin, Chelex, for $24 \mathrm{hr}$ at $37^{\circ} \mathrm{C}$. Sprague-Dawley rats were intratracheally dosed at day 0 with R-Total (1.0 mg/100 g body weight), R-Insoluble, R-Soluble, phosphate-buffered saline (Saline), Saline+Chelex (S-Chelex), or R-Soluble+Chelex (RChelex). At day $3,5 \times 10^{5}$ (high dose) or $5 \times 10^{4}$ (low dose) Listeria monocytogenes were intratracheally instilled into rats from each treatment group. At days 6, 8 and 10, left lungs were removed, homogenized, and cultured to assess bacterial clearance. The right lungs were preserved and stained with hematoxylin and eosin for histology. Pulmonary exposure of R-Total or R-Soluble before infection led to a marked increase in lung injury after inoculation, and an increase in morbidity in comparison to saline control rats. Treatment with R-Insoluble, S-Chelex, or R-Chelex caused no significant increases in lung damage and morbidity when compared to controls. Animals treated with R-Total or $\mathrm{R}$-Soluble had significantly more bacteria in the lungs at all time points after infection with the high dose of bacteria, and at days 6 and 8 after infection with the low dose of bacteria, indicating the inability of the lungs of these groups to effectively respond to the infection. Removal of the soluble metals from the R-Soluble sample with Chelex abolished the increase in bacterial burden observed in the R-Soluble group. Rats treated with R-Insoluble, S-Chelex, or R-Chelex showed no significant impairments in bacterial clearance when compared to saline controls. These results suggest that soluble metals present in ROFA may play a key role in increased susceptibility to pulmonary infection in exposed populations. 


\section{Introduction}

Epidemiology studies have demonstrated a positive correlation between increased levels of particulate air pollution and elevated morbidity and mortality in susceptible human populations (Dockery and Pope, 1994; Pope et al., 1995; Schwartz, 1994). Occupational exposure to residual oil fly ash (ROFA) has also been associated with adverse respiratory health effects in humans (Hauser et al., 1995a; 1995b; Levy et al., 1984). Increased infectivity and susceptibility to infection have been correlated with high levels of outdoor air pollutants (American Thoracic Society, 1996a; 1996b) as well. Upper and lower respiratory tract infections that interfere with normal activity have been observed after episodes of elevated air pollution levels. One such particulate pollutant, ROFA, is released into the environment from the combustion of fossil fuels and contributes approximately $2.5 \times 10^{5}$ tons to the ambient air particulate matter (PM) burden in the United States annually (Costa and Dreher, 1997). ROFA is a complex mixture of metals, sulfates, acids, fuel contaminants, and other unknown components combined with an insoluble, particulate carbon core (Fisher et al., 1983; Ghio et al., 2002).

Some of the adverse respiratory effects have been attributed to the metal component of various PM pollutants. Increased airway inflammation and oxidant production have been observed in humans after respiratory exposure to the PM with high metal levels (Ghio, 2004; Schaumann et al., 2004; Soukup et al., 2000). In vivo and in vitro studies have also shown that the metals present in particulate pollutants can lead to, but are not limited to, effects such as increased lung cell injury and inflammation (Kodavanti et al., 2001; Rice et al., 2001), increased cardiopulmonary injury (Costa and 
Dreher, 1997), altered cytokine production (Carter et al., 1997; Hetland et al., 2001), and increased oxidative stress and damage (Ghio et al., 1999; Kim et al., 2003).

Animal studies have shown that respiratory exposure to ROFA (Antonini et al., 2002; Hatch et al., 1985; Pritchard et al., 1996) and ambient air particulates (Zelikoff et al., 2002) that have a high metal content increases susceptibility to infection. ROFA has also been shown to decrease bactericidal activity of alveolar macrophages (Antonini et al., 2002). In vivo studies have demonstrated that the bioavailability of soluble transition metals in ROFA may be a potential mechanism of lung injury (Dreher et al., 1997; Gavett et al., 1997; Lewis et al., 2003). Soluble metals associated with ROFA have been implicated in the induction of protein leakage and increased influx of neutrophils and macrophages into the lungs (Kodavanti et al., 1998), as well as enhanced allergic hypersensitivity (Lambert et al., 2000), altered cytokine production, and increased epithelial cell injury (Dye et al., 1999).

The objective of the current study was to determine what component of ROFA may affect the susceptibility to pulmonary infection. We hypothesized that soluble metals associated with ROFA would suppress lung defense responses and slow clearance of bacteria from the lungs. The ROFA preparations used in this study were characterized for both size and metal composition. Rats were then pre-exposed to ROFA by intratracheal instillation. In addition, the ROFA sample was fractionated, and rats were intratracheally treated with the water-soluble or insoluble fractions. Following exposure to the ROFA samples, rats were intratracheally inoculated with Listeria monocytogenes at a high $\left(5 \times 10^{5}\right)$ or low $\left(5 \times 10^{4}\right)$ dose of bacteria. Animal weight, lung injury, and pulmonary clearance of L. monocytogenes after ROFA exposure were assessed. To determine the 
effects of the soluble metals on these parameters, metals were then removed from the soluble sample with a chelating agent, Chelex, and a group of animals was exposed to the chelated soluble sample of ROFA, followed by bacterial inoculation. 


\section{Methods}

Animals - Male Sprague-Dawley [Hla:(SD)CVF] rats (Hilltop Laboratories, Scottdale, PA) weighing 250-300 g, approximately 10 weeks old, were used for all experiments. They were given the ProLab 3500 diet and tap water ad libitum, housed in a clean air and viral- and antigen-free room with restricted access in an Association for Assessment and Accreditation of Laboratory Animal Care (AAALAC) -approved animal facility, and allowed to acclimate for one week before use. The rats were monitored and found to be free of endogenous viral pathogens, parasites, mycoplasms, Helicobacter and CAR Bacillus.

Materials - L. monocytogenes (strain 10403S, serotype 1) was obtained as a gift from Dr. Rosana Schafer of the Department of Microbiology and Immunology at West Virginia University. Residual oil fly ash (ROFA) was collected from a precipitator at Boston Edison Co., Mystic Power Plant \#4, Everett, MA. The chelating resin, Chelex 100 (iminodiacetic acid), was purchased from Sigma-Aldrich Co., St. Louis, MO.

Experimental Design - At day 0, animals were pre-exposed to ROFA samples or phosphate-buffered saline (PBS) (vehicle control) by intratracheal instillation. At day 3, the animals were inoculated via intratracheal instillation with $5 \times 10^{5}$ or $5 \times 10^{4} \mathrm{~L}$. monocytogenes. At days 6, 8, and 10, the rats were euthanized, and the left lungs of animals were removed, homogenized, and the number of colony forming units (CFUs) was counted. The right lungs of the same animals were fixed in formalin for histology.

ROFA Characterization - Particle size of the ROFA sample was determined by scanning electron microscopy (SEM) (JSM-\#5600 SEM, JEOL Ltd., Peabody, MA) and previously characterized (Antonini et al., 2002). ROFA particles were of respirable size 
with a count mean diameter of $2.2 \mu \mathrm{m}$. The metal constituents of the ROFA samples were analyzed using inductively coupled argon plasma, atomic-emission spectroscopy (NIOSH, 1994).

ROFA Treatment - The ROFA sample (R-Total) was suspended in sterile PBS (6 mg/ml), sonicated for 1 min with a Sonifier 450 Cell Disruptor (Branson Ultrasonics, Danbury, CT), and allowed to shake and incubate for $24 \mathrm{hr}$ at $37^{\circ} \mathrm{C}$. The sample was further divided into soluble and insoluble components by centrifugation at 12,000 $\mathrm{g}$ for $30 \mathrm{~min}$. The supernatant of the sample was recovered and filtered (R-Soluble). The pellet was resuspended in saline (R-Insoluble). Chelex was added to the R-Soluble sample or to saline (20 mg Chelex/ $0.1 \mathrm{mg}$ ROFA), and incubated on a rotor overnight. The samples were centrifuged and the supernatant of the R-Soluble sample (R-Chelex) and the saline sample (S-Chelex) were recovered. To control for the acidity of the ROFA sample, a solution of $0.63 \mathrm{mM} \mathrm{H}_{2} \mathrm{SO}_{4}$ was prepared in saline and adjusted to a $\mathrm{pH}$ of 4.0 with $\mathrm{HCl}$. Rats were lightly anesthetized by an intraperitoneal injection of $0.6 \mathrm{ml}$ of a $1 \%$ solution of sodium methohexital (Brevital, Eli Lilly, Indianapolis, IN) and intratracheally instilled with $1.0 \mathrm{mg} / 100 \mathrm{~g}$ body weight (bw) of ROFA in $300 \mu \mathrm{l}$ of PBS, according to the method of Brain et al. (1976). In addition, R-Soluble, R-Insoluble, R-Chelex, or SChelex were administered by intratracheal instillation using quantities equivalent to those in the R-Total instillate. Animals in the vehicle control group were intratracheally dosed with $300 \mu \mathrm{l}$ of sterile PBS (Saline). The ROFA dose chosen was previously shown to induce inflammation (Antonini et al., 2002), and fell within the range of concentrations consistently used in other animal studies evaluating the pulmonary responses to ROFA (Pritchard et al., 1996; Dreher et al., 1997; Gavett et al., 1997; Kodavanti et al., 1998). 
Intratracheal Bacteria Inoculation - L. monocytogenes was cultured overnight in brain-heart infusion broth (Difco Laboratories, Detroit, $\mathrm{MI}$ ) at $37^{\circ} \mathrm{C}$ in a shaking incubator. Following incubation, the bacterial concentration was determined spectrophotometrically at an optical density of $600 \mathrm{~nm}$. The sample was diluted to the

concentration of $5 \times 10^{5}$ or $5 \times 10^{4}$ L. monocytogenes in $500 \mu$ of sterile PBS. The bacterial dose was intratracheally instilled 3 days post-ROFA sample instillation. At this time point, the initial effects associated with a bolus I.T. dose of a substance, which can occur in the first day after instillation, have subsided. The doses of $L$. monocytogenes chosen were found to give a uniform infection and did not result in mortality in untreated naïve Sprague-Dawley rats in a previous study (Antonini et al., 2001). To quantify the exact number of bacteria instilled on day 3, the instillate was cultured on brain-heart infusion agar overnight at $37^{\circ} \mathrm{C}$ and colony forming units (CFUs) were counted.

Morbidity/Histolopathology - Animal weights were monitored over the course of the treatment period as an indicator of morbidity. Histology was performed on the right lungs of the rats from each treatment group. Rats were euthanized with an overdose of sodium pentobarbital, the left bronchus was clamped off, and the right lungs were preserved with $10 \%$ neutral buffered formalin by airway fixation at $30 \mathrm{~cm}$ water pressure. The right lobes of the lungs were removed, embedded in paraffin, sectioned, and stained with hematoxylin and eosin (H\&E). Stained tissue sections were imaged with a SprintScan 35 Plus slide scanner (Polaroid, Waltham, MA).

Pulmonary Clearance of $\boldsymbol{L}$. monocytogenes - At days 6, 8, and 10, the left lungs were removed from all the rats in each treatment group. The excised tissues were suspended in $10 \mathrm{ml}$ of sterile water, homogenized using a PowerGen 700 homogenizer 
(Fisher Scientific, Pittsburgh, PA), and cultured quantitatively on brain-heart infusion agar plates (Becton Dickinson and Co., Cockeysville, MD). The number of viable CFUs from the left lung homogenate was counted after an overnight incubation at $37^{\circ} \mathrm{C}$.

Statistical Analysis - The number of animals for each treatment group at each time point post-infection is presented in Table 1.1. Results are expressed as means \pm standard error of measurement (SE). Statistical analyses were carried out with the SigmaStat 3.1 statistical program (Chicago, IL). The significance of the interaction among different treatment groups for the different parameters at each time point was assessed using analysis of variance (ANOVA). The significance of difference between individual groups was analyzed using the Student-Newman-Keuls method with the criterion of significance set at $p<0.05$. 


\section{$\underline{\text { Results }}$}

ROFA Characterization - SEM showed that the particles ranged in size from approximately $1-20 \mu \mathrm{m}$ (Figure 1.1) and that the particles were found to have a count mean diameter of $2.2 \mu \mathrm{m}$. Elemental analysis was performed on the R-Total, R-Insoluble, R-Soluble, and R-Chelex samples to determine their compositions (Table 1.2).

Significant amounts of $\mathrm{Fe}, \mathrm{Al}, \mathrm{V}, \mathrm{Ni}$, and $\mathrm{Ca}$, as well as a small amount of $\mathrm{Zn}$, were present in the $\mathrm{R}$-Total sample and the $\mathrm{pH}$ of the sample was found to be acidic. R-Total was separated into soluble and insoluble fractions. The insoluble fraction, with a relatively neutral $\mathrm{pH}$, consisted primarily of $\mathrm{Fe}, \mathrm{V}$, and $\mathrm{Al}$, whereas the soluble fraction, with an acidic $\mathrm{pH}$, contained significant amounts of $\mathrm{Ni}, \mathrm{Al}, \mathrm{Ca}, \mathrm{Fe}$, and $\mathrm{Zn}$. Chelation of R-Soluble with Chelex resulted in removal of the majority of soluble metals with the exception of a small amount of Fe and V.

Morbidity: ROFA Fractions - Morbidity in animals pre-treated with Saline, RTotal, R-Soluble, or R-Insoluble was monitored as the \% change in body weight gained or lost after infection with the high dose of L. monocytogenes (Figure 1.2A) and the low dose (Figure 1.2B). Body weight of animals instilled with R-Insoluble prior to infection with either bacteria dose did not differ from the saline controls across the course of the infection. Rats treated with R-Total and R-Soluble lost significantly more body weight after inoculation with the high dose of bacteria when compared to the Saline and RInsoluble groups at all time points post-infection with the most progressive loss attributed to the R-Soluble-treated rats. Unexpectedly, a number of animals from the R-Soluble and R-Total groups did not survive the course of the infection with the high dose of bacteria. When exposed to the low dose of L. monocytogenes, rats pre-treated with R-Soluble 
showed a significant decrease in body weight at Day 6, 8, and 10, and at Day 6 for the animals treated with R-Total. However, animals from the R-Total and R-Soluble group were able to regain body weight across the time course of the infection after the low dose of bacteria indicating the better ability of these groups to recover from that dose when compared to the high dose of bacteria. Body weight of uninfected animals treated with Saline, R-Total, and R-Soluble were monitored to determine if treatment with the samples resulted in disparities in weight at the time of infection (Table 1.3). Rats from all groups had gained rather than lost body weight by Day 3 after treatment with the ROFA samples. Uninfected rats from all groups continued to gain weight through the time course, with no significant differences found among treatment groups.

Pulmonary Bacterial Clearance: ROFA Fractions - Losses in body weight in the R-Total and R-Soluble groups were accompanied by decreased clearance of bacteria from the lungs (Figure 1.3). Animals treated with R-Insoluble and Saline were able to clear the majority of bacteria from the lungs by day 10 at both the high (Figure 1.3A) and low dose (Figure 1.3B) of bacteria, whereas animals pre-exposed to R-Total or R-Soluble had a significantly greater bacterial lung burden at the early time points post-infection. At the high dose, the number of CFUs in the left lungs of rats treated with R-Total or RSoluble was approximately 200-fold greater than Saline and R-Insoluble on day 10. Number of bacterial CFUs in the lungs of the R-Total and R-Soluble reported in figure 1.3 represent the fraction of animals that survived to those time points. Due to the unexpected decrease in survival, the bacterial lung burdens reported at days 8 and 10 likely underestimate the extent of the infection in those groups. Despite the significant decrease in bacterial clearance at the early time points post-infection with the low dose of 
bacteria, animals pre-treated with R-Soluble and R-Total were able to clear the majority of bacteria from the lungs by Day 10 and did not differ from Saline- or R-Insolubletreated animals at that time point.

The R-Total and R-Soluble samples were found to be acidic with the $\mathrm{pH}$ of 5.0 and 4.1, respectively. The bacterial lung burden of rats pre-treated with a solution of $0.63 \mathrm{mM} \mathrm{H}_{2} \mathrm{SO}_{4}$ at a pH of 4.0, and inoculated with the high dose of L. monocytogenes did not differ from saline controls (Figure 1.4).

Pulmonary Infection: Metal Chelation - R-Soluble slowed the clearance of both the high and low dose of bacteria from the lungs, whereas animals treated with Saline, SChelex, or R-Chelex were able to clear the majority of bacteria from the lungs by day 10 and did not differ significantly from each other at any time point post-infection (Figure $1.5 \mathrm{~A} \& \mathrm{~B})$. Animals pre-exposed to R-Soluble, followed by the high dose of bacteria (Figure 1.5A) had a significantly greater bacterial lung burden at days 6,8 and 10 compared to the saline and chelated controls. At the low dose of bacteria (Figure 1.5B), bacterial clearance in the R-Soluble group was significantly decreased compared to all groups at Day 6, and at Day 8 and 10 when compared to the Saline group.

Lung Histopathology - H\&E-stained tissue sections from the lower right lung of animals treated with R-Soluble, R- Chelex, or Saline, followed by inoculation with $5 \times 10^{5}$ L. monocytogenes (high dose) were scanned at days 6 and 10 (Figure 1.6). Infection with L. monocytogenes led to edema, inflammation, and the formation of granulomatous lesions (areas of dark purple) characterized by the presence of amorphous tissue debris in the lungs of Saline and R-Chelex groups. The lesions that were observed in the lungs of rats that were pre-treated with R-Soluble were more extensive and significantly more 
pronounced than those observed in the other groups. At the high dose, lung injury and inflammation in the R-Soluble group was still progressing at day 10, whereas the lung response of the other groups had subsided by this time point. 


\section{$\underline{\text { Discussion }}$}

ROFA is a particulate pollutant emitted into the environment as a product of the combustion of fossil fuel. The composition of ROFA consists of a complex mixture of metals and other fuel contaminants complexed to an insoluble carbon core (Fisher et al., 1983). ROFA is known to induce a variety of respiratory responses in vivo and in vitro including, but not limited to, alterations in transcription factor activation (Samet et al., 2002), increased airway hyperreactivity (Goldsmith et al., 1999; Hamada et al., 1999), increased mucin secretion (Jiang et al., 2000; Longphre et al., 2000), increased oxidative stress (Becker et al., 2002; Ghio et al., 2002; Huang et al., 2003; Pritchard et al., 1996), altered cytokine expression (Carter et al., 1997; Tao and Kobzik, 2002), and altered gene expression (Nadadur et al., 2000; Nadadur and Kodavanti, 2002). The presence of soluble transition metals of ROFA has been implicated in the development of adverse respiratory responses, and has been shown to play a role in lung inflammation and injury (Dreher et al., 1997; Dye et al., 1999; Gavett et al., 1997; Ghio et al., 1999; Kodavanti et al., 1998; Lewis et al., 2003).

The metal composition of ROFA is dependent on the fuel source, the combustion conditions in the power plant (including temperature, pressure changes, gas velocities, and duct work), and efficiency of emission control devices (Burckle, 1977; El-Mogazi et al., 1988). Responses to ROFA may vary depending on the composition of soluble metals as well as their interaction with each other. Kodavanti et al. (1997) found that the metal mixture of ROFA, as well as soluble Ni, Fe, and V (three predominant water-leachable metals), induced lung injury and enhanced cytokine induction (interleukin-1 and -5 , and macrophage inflammatory protein-2). They also showed that $\mathrm{Ni}$ alone caused the most 
severe damage while Fe and V alone produced a less severe pathology. Dreher et al. (1997) demonstrated that the individual soluble metals within a ROFA mixture may interact in an antagonistic fashion, specifically Ni-induced lung injury was diminished in the presence of soluble Fe or soluble V. Ni has also been shown to be a suppressor of alveolar macrophage (AM) function whereas $\mathrm{V}$ appears to produce a greater oxidative burst in AMs (Graham et al., 1975; Kodavanti et al., 2002). The individual soluble metals of ROFA may have differential effects on lung responses. Therefore, ROFA from various sources that differ in chemical composition may induce different effects. It is of interest to note that the ROFA used in the present study contained significant amounts of soluble $\mathrm{Ni}, \mathrm{Fe}$, and $\mathrm{Al}$; however, very little $\mathrm{V}$ was soluble. Based on observations from previous studies, it is likely that these soluble metals may suppress lung defense responses.

The objective of this study was to determine the component of ROFA that may affect the susceptibility to pulmonary infection. Animals were exposed to ROFA or its soluble or insoluble fractions, followed by pulmonary infection with $L$. monocytogenes. L. monocytogenes is a gram-positive, facultative intracellular bacteria that has been commonly used to assess pulmonary defense function (Antonini et al., 2000; Cohen et al., 2001; Jakab et al., 1993; Van Loveren et al., 1988). An effective response to $L$. monocytogenes requires the proper cross-talk between the innate immune system (macrophages, neutrophils, natural killer cells) and the cell-mediated adaptive immune system $\left(\mathrm{CD}^{+} \mathrm{T}\right.$-cells, $\mathrm{T}_{\mathrm{H}} 1$ cells $)$.

The major findings of this study confirmed our hypothesis that the soluble metals associated with ROFA would lead to increased morbidity and infectivity. ROFA and the associated soluble metals caused a greater infection at both doses of L. monocytogenes 
resulting in decreased body weight and increased bacterial lung burden. Upon removal of the soluble metals from the sample, changes in body weight and clearance were restored to control levels. Antonini et al. (2002) showed that ROFA may increase susceptibility to infection by decreasing AM bactericidal activity and the production of nitric oxide (an antimicrobial agent as well as a second messenger) by AMs. The effects of ROFA on AMs may alter innate immune responses and consequently affect adaptive immunity and the ability of the animal to respond to the infection.

The present study demonstrated that the soluble fraction of ROFA likely contains elements that are responsible for the increased susceptibility to infection. Zelikoff et al. (2002) exposed Streptococcus pneumoniae-infected rats to soluble Fe, Mn, and Ni to examine the effect of metals commonly found in ambient air pollution on immunotoxicity. The investigators found that both $\mathrm{Ni}$ and Fe reduced pulmonary clearance of the bacteria in infected rats. Furthermore, soluble Fe was shown to possibly affect both innate and adaptive immunity in uninfected rats as evidenced by changes in blood lymphocyte profiles and an inhibition of lymphocyte proliferation upon challenge. T-cells (cell-mediated immunity) proved to be more sensitive to inhalation of soluble $\mathrm{Ni}$ than did B-lymphocytes (humoral immunity), whereas soluble Fe had suppressive effects on both populations of lymphocytes. The ROFA used in our study contained a significant amount of soluble $\mathrm{Ni}$ and $\mathrm{Fe}$, and it is possible that these metals altered the function of cells involved in both adaptive immune response and innate immune responses to infection.

In summary, soluble metals associated with environmental and occupational particulates likely alter pulmonary defense and increase susceptibility to infection. It has 
been demonstrated that the soluble metals associated with ROFA increase morbidity and decrease pulmonary clearance of bacteria. Additional studies were performed, as described in study 2 , that evaluated the role of individual soluble metals, as well as the mechanisms of their actions, in lung defense against bacteria infection. 


\section{Figures and Tables}
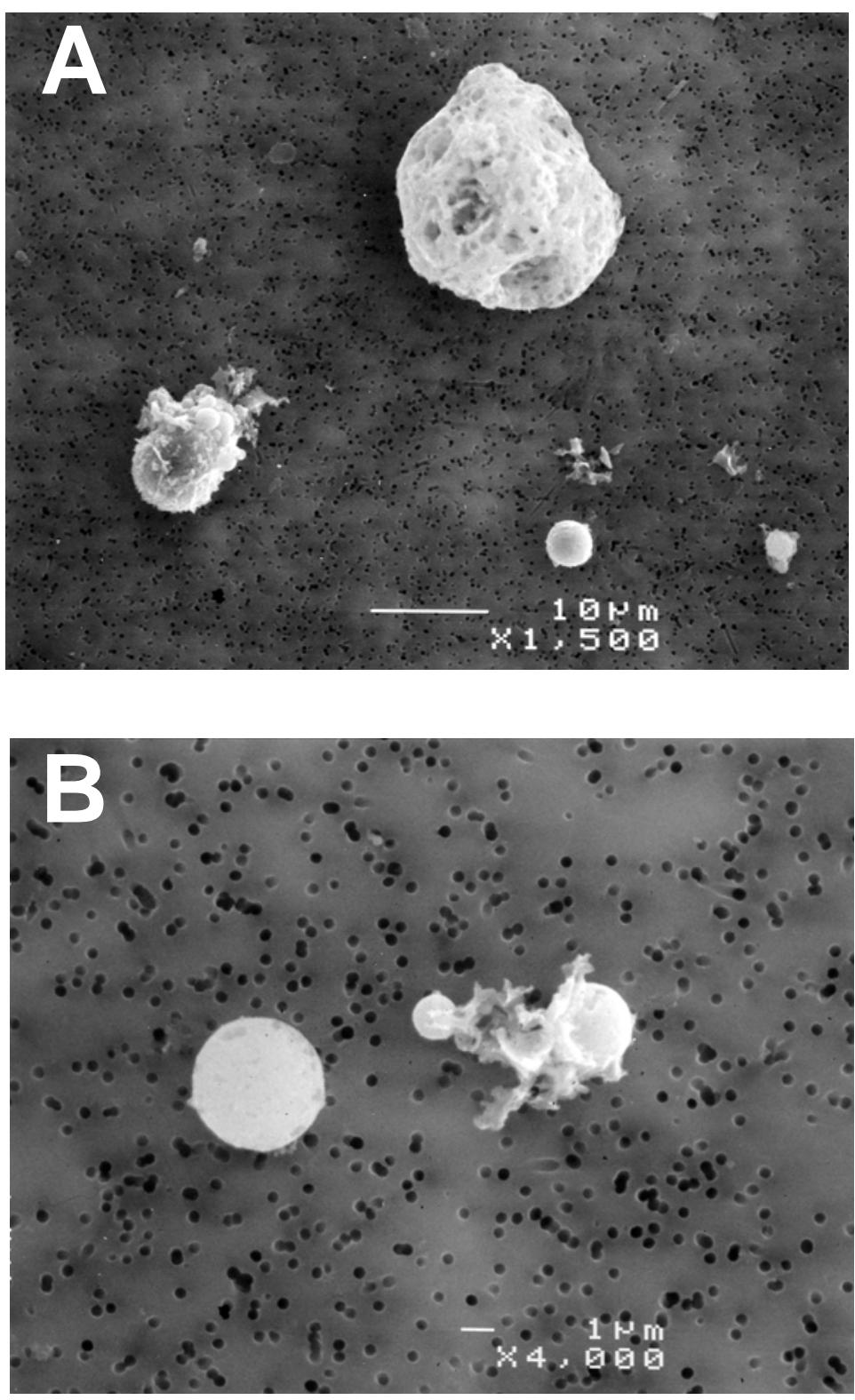

Figure 1.1. Scanning electron micrographs of ROFA particles representing the size distribution $(\mathrm{A} \& \mathrm{~B})$. The count mean diameter was determined to be approximately $2.2 \mu \mathrm{m}$. 

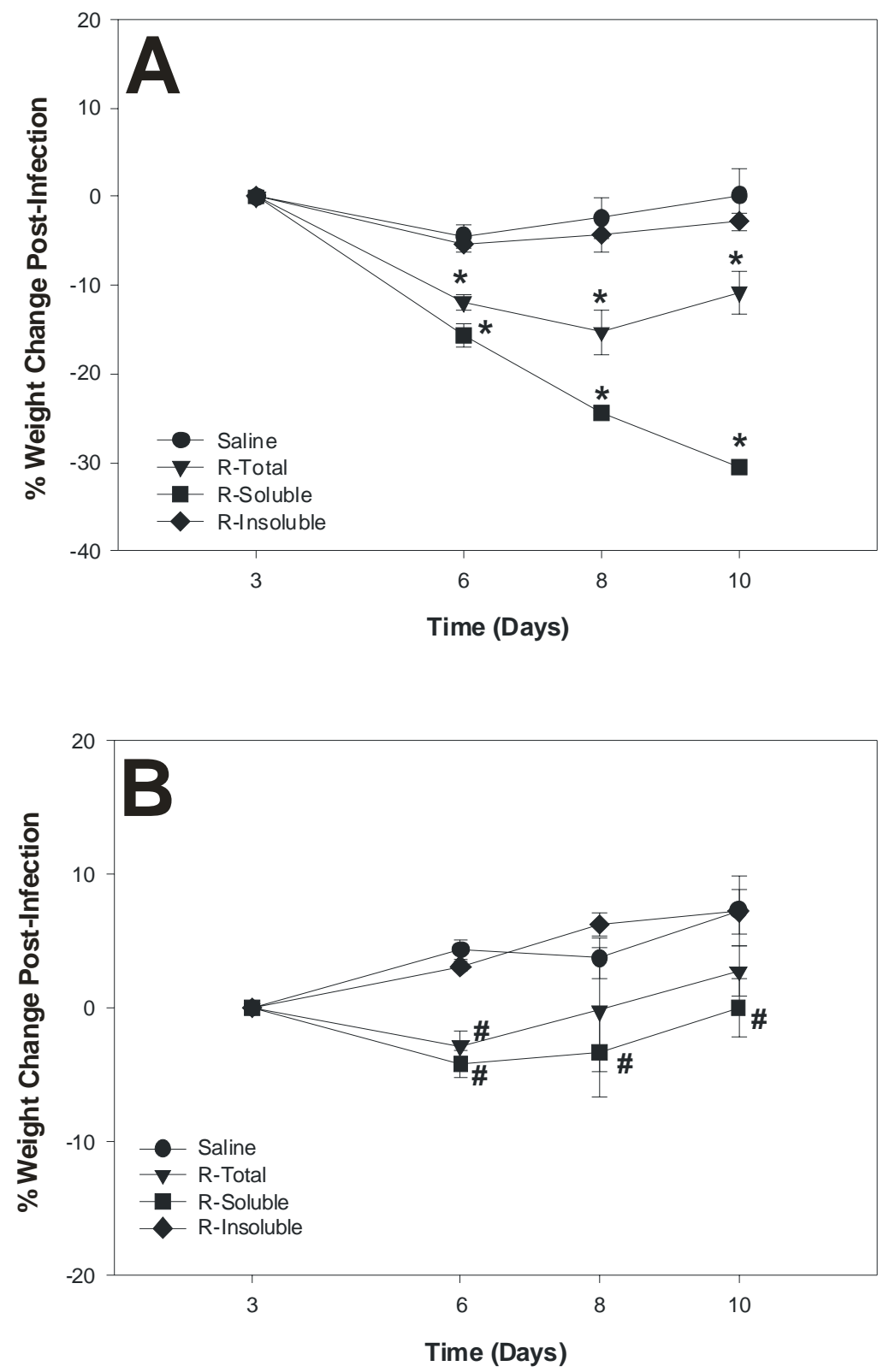

Figure 1.2. \% change in body weight post-infection of rats that were pre-exposed to $\mathrm{R}$-Total, R-Soluble, R-Insoluble, or Saline by intratracheal instillation 3 days prior intratracheal inoculation with $5 \times 10^{5}$ (A) or $5 \times 10^{4}$ (B) L. monocytogenes. Values are means $\pm \mathrm{SE}, *$ significantly different from all groups, \# significantly different from RInsoluble and Saline groups $(p<0.05)$. 

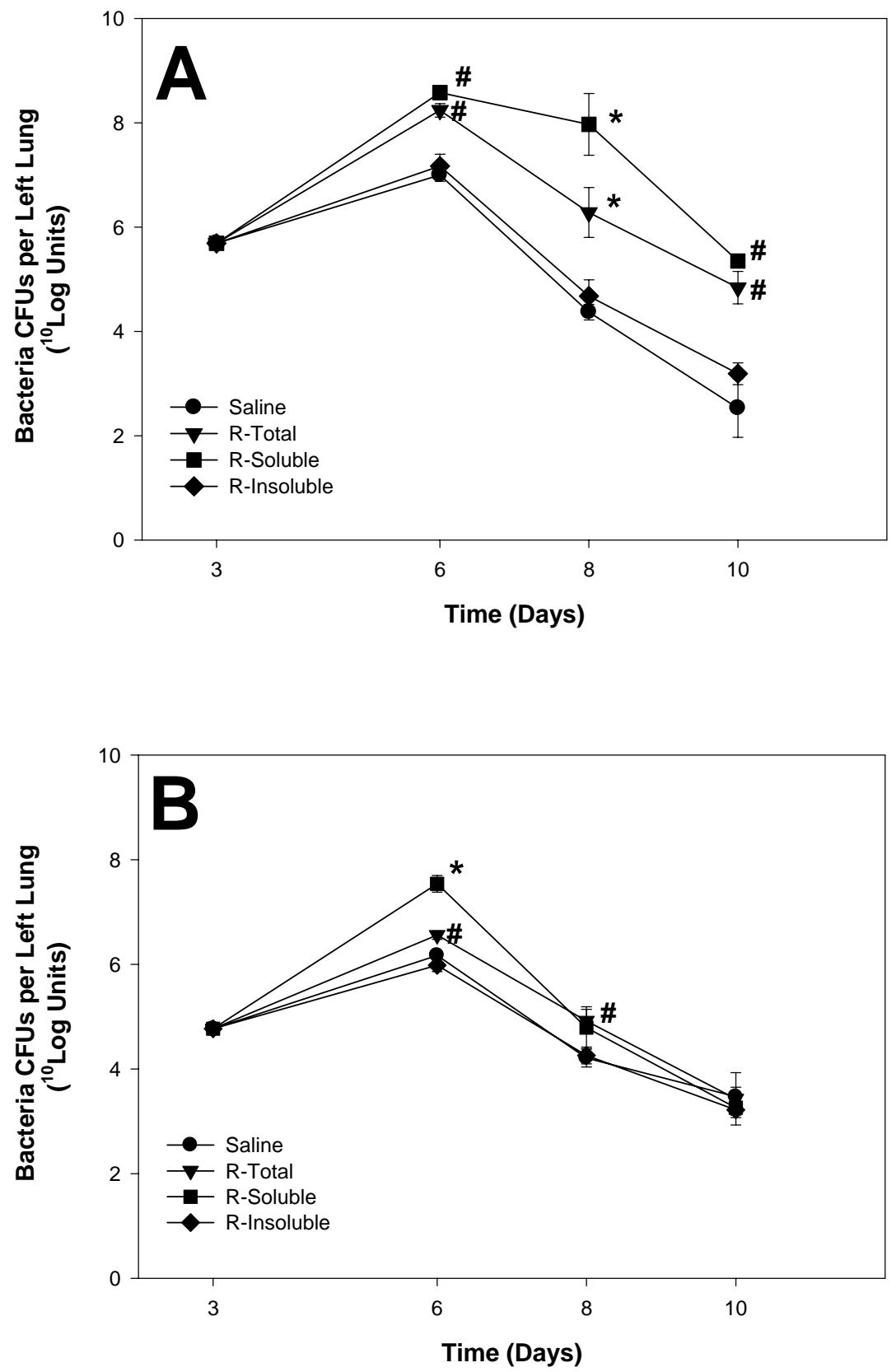

Figure 1.3. Number of bacterial CFUs in the left lungs of rats that were pre-exposed to R-Total, R-Soluble, R-Insoluble, or Saline by intratracheal instillation 3 days prior to intratracheal inoculation with $5 \times 10^{5}$ (A) or $5 \times 10^{4}$ (B) L. monocytogenes. Bacterial CFUs on instilled on day 3 were quantified after an overnight culture of the instillate. Values are means $\pm \mathrm{SE}, *$ significantly different from all groups, \# significantly different from RInsoluble and Saline groups $(p<0.05)$. 


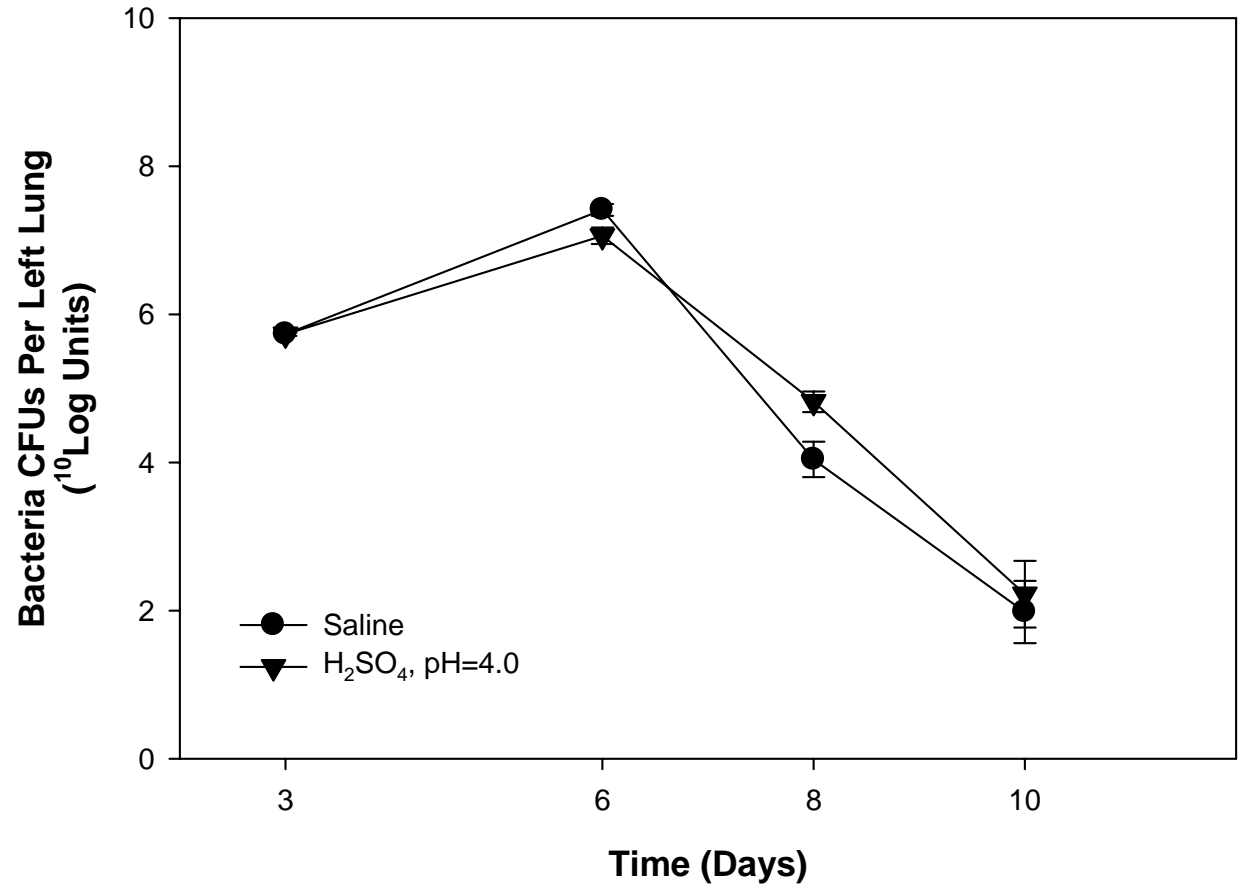

Figure 1.4. Number of bacterial CFUs in the left lungs of rats that were pre-exposed to an acidic solution $\left(0.63 \mathrm{mM} \mathrm{H}_{2} \mathrm{SO}_{4}, \mathrm{pH}=4.0\right)(\mathrm{n}=10$ per time point) or Saline ( $\mathrm{n}=6$ per time point) by intratracheal instillation 3 days prior to intratracheal inoculation with the high dose $\left(5 \times 10^{5}\right)$ of L. monocytogenes. Bacterial CFUs on instilled on day 3 were quantified after an overnight culture of the instillate. Values are means \pm SE. 

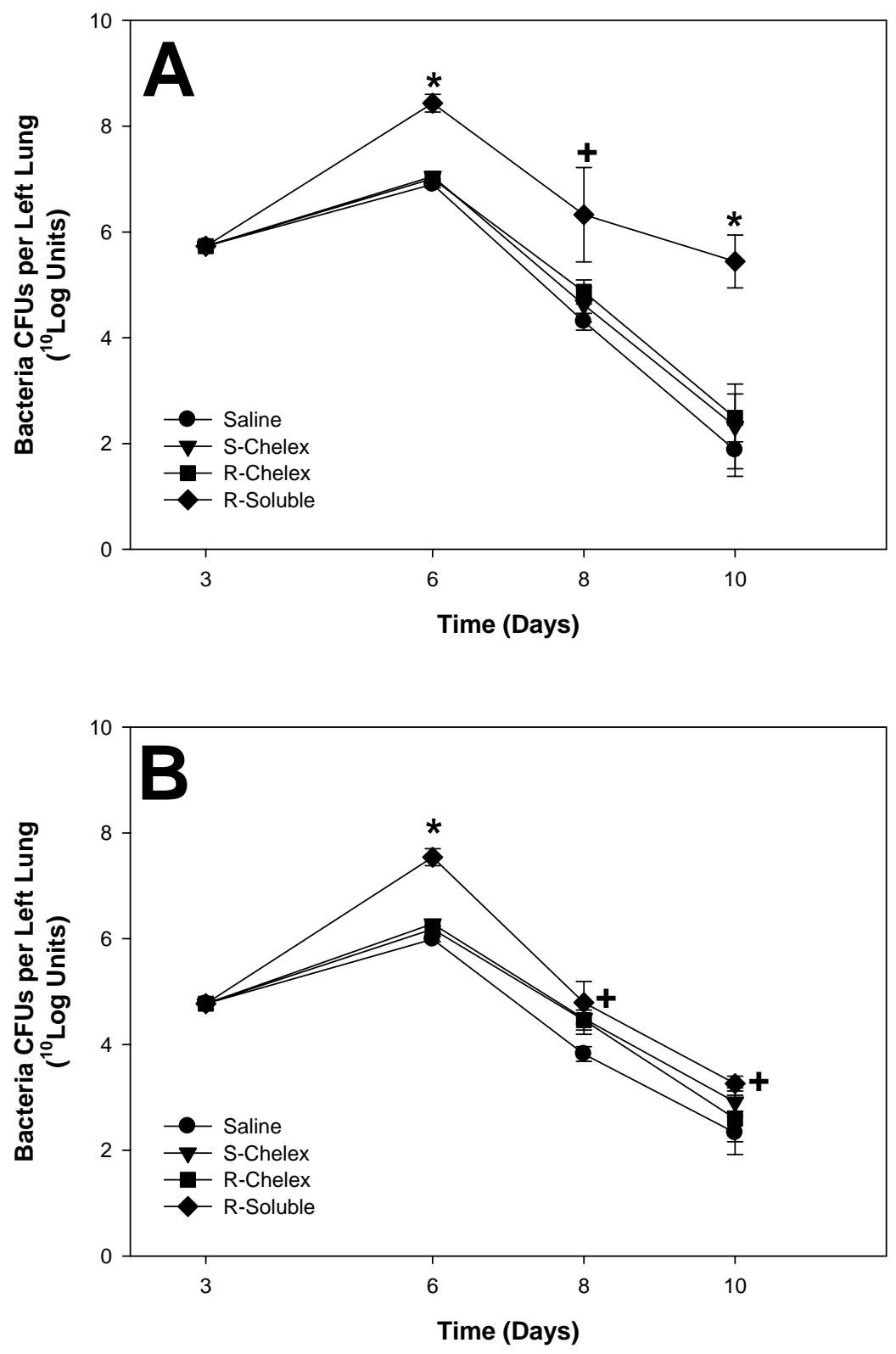

Figure 1.5. Number of bacterial CFUs in the left lungs of rats that were pre-exposed to R-Soluble, R-Chelex, S-Chelex, or Saline by intratracheal instillation 3 days prior to intratracheal inoculation with $5 \times 10^{5}$ (A) or $5 \times 10^{4}$ (B) L. monocytogenes. Bacterial CFUs on instilled on day 3 were quantified after an overnight culture of the instillate. Values are means $\pm \mathrm{SE}, *$ significantly different from all groups, + significantly different from Saline groups $(p<0.05)$. 


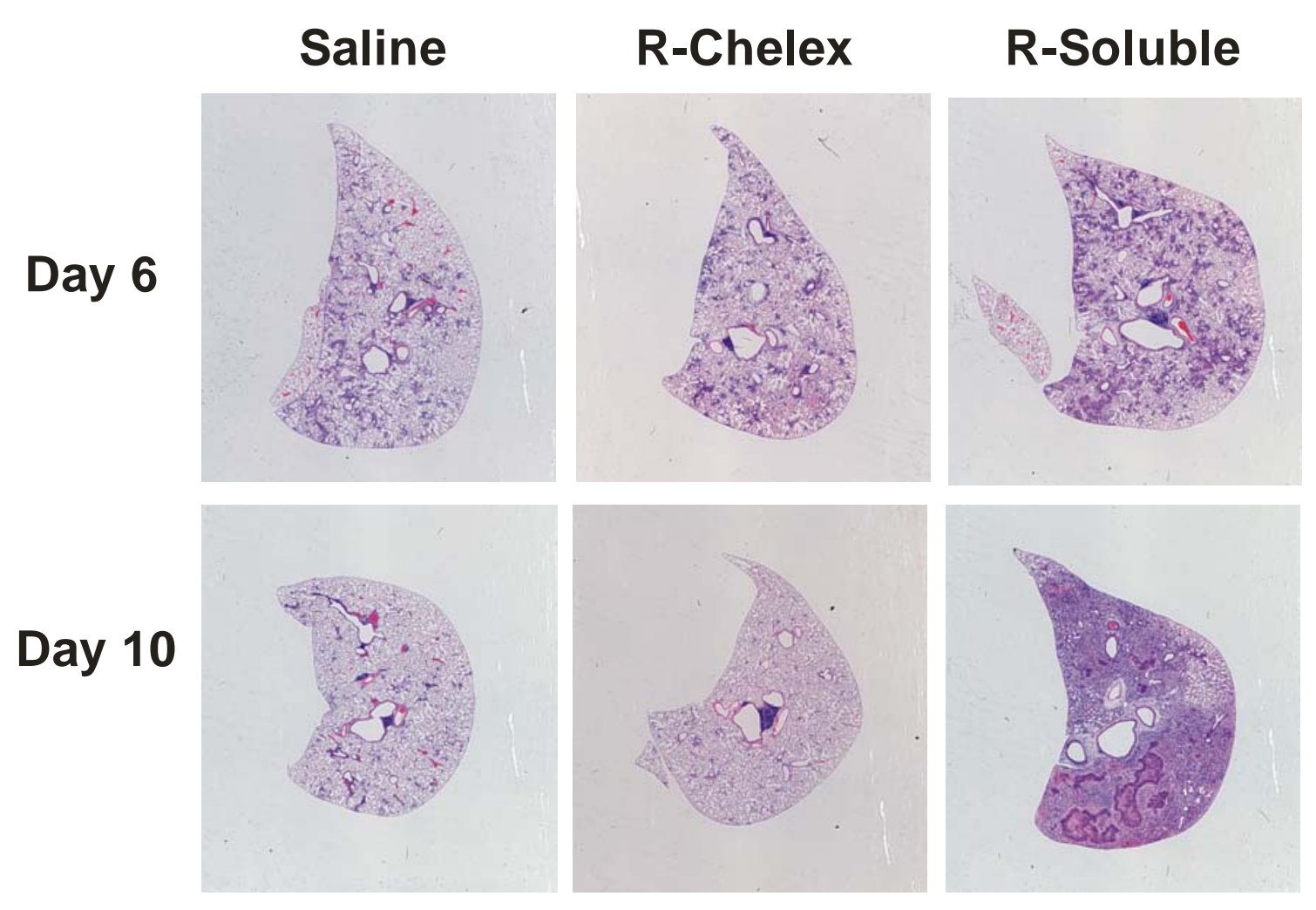

Figure 1.6. Whole slide scans of hematoxylin and eosin-stained right lung tissue sections from animals intratracheally instilled with R-Soluble, R-Chelex, or Saline followed by inoculation with $5 \times 10^{5} \mathrm{~L}$. monocytogenes at days 6 and 10 . 
Table 1.1. Number of Animals per Treatment Group per Time Point

High Dose of Listeria Low Dose of Listeria

\begin{tabular}{|c|ccc|ccc|}
\hline Treatment & Day 6 & Day8 & Day10 & Day 6 & Day8 & Day10 \\
\hline R-Total & 19 & 12 & 9 & 8 & 10 & 10 \\
\hline R-Soluble & 27 & 10 & 7 & 10 & 10 & 10 \\
\hline R-Chelex & 19 & 17 & 11 & 10 & 10 & 10 \\
\hline R-Insoluble & 7 & 7 & 8 & 4 & 4 & 4 \\
\hline Saline & 27 & 25 & 15 & 10 & 9 & 10 \\
\hline S-Chelex & 6 & 6 & 4 & 4 & 4 & 4 \\
\hline
\end{tabular}

Table 1.1. Number of animals per treatment group for each time point. All animals were inoculated with $L$. monocytogenes on Day $3 . \mathrm{R}=\mathrm{ROFA} ; \mathrm{S}=$ Saline. 
Table 1.2. Element Mass ( $\mu \mathrm{g} / 2 \mathrm{mg}$ instillate)

\section{$\underline{\text { R-Total(pH=5.0) }} \underline{\text { R-Insoluble(pH=7.06) }} \quad \underline{\text { R-Soluble(pH=4.1) }} \quad \underline{\text { R-Chelex(pH=9.7) }}$}

$\begin{array}{llllc}\text { Fe } & 244 & 186 & 37.2 & 6.76 \\ \text { Al } & 121 & 64.1 & 46.6 & <0.004 \\ \text { V } & 92.0 & 83.1 & 1.17 & 0.574 \\ \text { Ni } & 76.9 & 11.0 & 55.7 & <0.002 \\ \text { Ca } & 61.1 & 7.16 & 45.1 & <0.045 \\ \text { Zn } & 10.7 & 1.13 & 8.69 & <0.005\end{array}$

Trace Elements: $\quad$ Ba, Cd, Co, Cr, Cu, Mn, Pb

Table 1.2. Element mass in $\mu \mathrm{g}$ amount present in the $2 \mathrm{mg}$ instillate of the R-Total, RInsoluble, R-Soluble, and R-Chelex samples as determined by inductively coupled argon plasma, atomic-emission spectroscopy. 
Table 1.3. \% Weight Gain Post-ROFA Treatment - No Listeria

\begin{tabular}{|ccccc|}
\hline Treatment & Day 3 & Day 6 & Day 8 & Day 10 \\
\hline Saline & $7.67 \pm 0.71$ & $14.6 \pm 1.85$ & $18.5 \pm 0.51$ & $16.5 \pm 2.34$ \\
\hline R-Chelex & $6.31 \pm 0.45$ & $15.9 \pm 1.54$ & $14.6 \pm 3.23$ & $16.8 \pm 4.81$ \\
\hline R-Soluble & $6.29 \pm 0.78$ & $12.8 \pm 1.40$ & $15.4 \pm 1.67$ & $15.2 \pm 2.59$ \\
\hline R-Total & $4.13 \pm 0.32$ & $13.8 \pm 1.60$ & $14.3 \pm 1.74$ & $12.4 \pm 3.14$ \\
\hline
\end{tabular}

Table 1.3. $\%$ of total body weight gained by uninfected rats on days $3,6,8$, and 10 after exposure to Saline $(n=4)$, R-Chelex $(n=4)$, R-Soluble $(n=4)$, or R-Total $(n=4)$. 


\section{References}

American Thoracic Society, Committee of the Environmental and Occupational Health Assembly, Bascom, R., Bromberg, P. A., Costa, D. A., Devlin, R., Dockery, D. W., Frampton, M. W., Lambert, W., Samet, J. M., Speizer, F. E., and Utell, M. 1996a. Health effects of outdoor air pollution. Part 1. Am. J. Respir. Crit. Care Med. 153:3-50.

American Thoracic Society, Committee of the Environmental and Occupational Health Assembly, Bascom, R., Bromberg, P. A., Costa, D. A., Devlin, R., Dockery, D. W., Frampton, M. W., Lambert, W., Samet, J. M., Speizer, F. E., and Utell, M. 1996b. Health effects of outdoor air pollution. Part 2. Am. J. Respir. Crit. Care Med. 153:477-498.

Antonini, J. M., Yang, H-M., Ma, J. Y. C., Roberts, J. R., Barger, M. W., Butterworth, L., Charron, T. G., and Castranova, V. 2000. Subchronic silica exposure enhances respiratory defense mechanisms and the pulmonary clearance of $L$. monocytogenes in rats. Inhal. Toxicol. 12:1017-1036.

Antonini, J. M, Roberts, J. R., and Clarke, R. W. 2001. Strain-related differences of nonspecific respiratory defense mechanisms in rats using a pulmonary infectivity model.

Inhal. Toxicol. 13:85-102.

Antonini, J. M., Roberts, J. R., Jernigan, M. R., Yang, H-M., Ma, J. Y. C., and Clarke, R. W. 2002. Residual oil fly ash increases the susceptibility to infection and severely damages the lungs after pulmonary challenge with a bacterial pathogen. Toxicol. Sci. 70:110-119.

Becker, S., Soukup, J. M., and Gallagher, J. E. 2002. Differential particulate air pollution induced oxidant stress in human granulocytes, monocytes, and alveolar macrophages. Toxicol. In Vitro. 16:209-218.

Brain, J. D., Knudson, D. E., Sorokin, S. P., and Davis, M. A. 1976. Pulmonary distribution of particles given by intratracheal instillation or by aerosol inhalation. Environ. Res. 11:13-33.

Burckle, J. O. 1977. Particulate emission measurement models. In Energy Technology Handbook, ed. Considine, D., pp. 9.391-9.406. New York: McGraw-Hill.

Carter, J. D., Ghio, A. J., Samet, J. M., and Devlin, R. B. 1997. Cytokine production by human epithelial cells after exposure to an air pollution particle is metal-dependent. Toxicol. Appl. Pharmacol. 146:180-188.

Cohen, M. D., Sisco, M., Li, Y., Zelikoff, J. T., and Schlesinger, R. B. 2001. Ozoneinduced modulation of cell-mediated immune responses in the lungs. Toxicol. Appl. Pharmacol. 171:71-84. 
Costa, D. L., and Dreher, K. L. 1997. Bioavailable transition metals in particulate matter mediate cardiopulmonary injury in healthy and compromised animal models. Environ. Health Perspect. 105(Suppl. 5):1053-1060.

Dockery, D. W., and Pope III, C. A. 1994. Acute respiratory effects of particulate air pollution. Annu. Rev. Public Health. 15:107-132.

Dreher, K. L., Jaskot, R. H., Lehmann, J. R., Richards, J. H., McGee, J. K., Ghio, A. J., and Costa, D. L. 1997. Soluble transition metals mediate residual oil fly ash-induced acute lung injury. J. Toxicol. Environ. Health. 50:285-305.

Dye, J. A., Adler, K. B., Richards, J. H., and Dreher, K. L. 1999. Role of soluble metals in oil fly ash-induced airway epithelial injury and cytokine gene expression. Am J. Physiol. 277:L498-L510.

El-Mogazi, D., Lisk, D. J., and Weinstein, L. H. 1988. A review of physical, chemical, and biological properties of fly ash and effects on agricultural ecosystems. Sci. Total Environ. 74:1-37.

Fisher, G. L., McNeil, K. L., Prentice, B. A., and McFarland, A. R. 1983. Physical and biological studies of coal and oil fly ash. Environ. Health Perspect. 51:181-186.

Gavett, S. H., Madison, S. L., Dreher, K. L., Winsett, D. W., McGee, J. K., and Costa, D. L. 1997. Metal and sulfate composition of residual oil fly ash determines airway hyperreactivity and lung injury in rats. Environ. Res. 72:162-172.

Ghio, A. J., Stonehuerner, J., Dailey, L. A., and Carter, J. D. 1999. Metals associated with both the water-soluble and insoluble fractions of an ambient air pollutant particle catalyze an oxidative stress. Inhal. Toxicol. 11:37-49.

Ghio, A. J., Silbajoris, R., Carson, J. L., and Samet, J. M. 2002. Biologic effects of oil fly ash. Environ. Health Perspect. 110(Suppl. 1):89-94.

Ghio, A. J. 2004. Biological effects of Utah Valley ambient air particles in humans: a review. J. Aerosol Med. 17: 157-164.

Goldsmith, C. A., Hamada, K., Ning, Y. Y., Qin, P., Murthy, G. G. K., Lawrence, J. and Kobzik, L. 1999. Effects of environmental aerosols on airway hyperresponsiveness in a murine model of asthma. Inhal. Toxicol. 11:981-998.

Graham, J. A., Gardner, D. E., Waters, M. D., and Coffin, D. L. 1975. Effect of trace metals on phagocytosis by alveolar macrophages. Infect. Immun. 11:1278-1283.

Hamada, K., Goldsmith, C. A., and Kobzik, L. 1999. Increased airway hyperresponsiveness and inflammation in a juvenile mouse model of asthma exposed to air-pollutant aerosol. J. Toxicol. Environ. Health. 58:129-143. 
Hatch, G. E., Boykin, E., Graham, J. A., Lewtas, J., Pott, F., Loud, K., and Mumfor, J. L. 1985. Inhalable particles and pulmonary host defense: In vivo and in vitro effects of ambient air and combustion particles. Environ. Res. 36:67-80.

Huang, Y. C., Soukup, J., Harder, S. and Becker, S. 2003. Mitochondrial oxidant production by a pollutant dust and NO-mediated apoptosis in human alveolar macrophage. Am J. Physiol. 284:C24-C32.

Hauser, R., Elreedy, S., Hoppin, J. A., and Christiani, D. C. 1995a. Airway obstruction in boilmakers exposed to fuel oil ash: A prospective investigation. Am. J. Respir. Crit. Care Med. 152:1478-1484.

Hauser, R., Elreedy, S., Hoppin, J. A., and Christiani, D. C. 1995b. Upper airway response in workers exposed to fuel oil ash: Nasal lavage analysis. Occup. Environ. Med. 52:353-358.

Hetland, R. B., Myhre, O., Lag, M., Hongve, D., Schwarze, P. E., and Refsnes, M. 2001. Importance of soluble metals and reactive oxygen species for cytokine release induced by mineral particles. Toxicol. 165: 133-144.

Jakab, G. J. 1993. The toxicologic interactions resulting from inhalation of carbon black and acrolein on pulmonary antibacterial and antiviral defenses. Toxicol. Appl. Pharmacol. 121:167-175.

Jiang, N., Dreher, K. L., Dye, J. A., Li, Y., Richards, J. H., Martin, L. D., and Adler, K. B. 2000. Residual oil fly ash induces cytotoxicity and mucin secretion by guinea pig tracheal epithelial cells via an oxidant-mediated mechanism. Toxicol. Appl. Pharmacol. 163:221-230.

Kim, J. E., Hauser, R., Wand, M. P., Herrick, R. F., Amarasiriwardena, C. J., and Christiani, D. C. 2003. The association of expired nitric oxide with occupational particulate metal exposure. Environ. Res. 93: 158-166.

Kodavanti, U. P., Hauser, R., Christiani, D. C., Meng, Z. H., McGee, J., Ledbetter, A., Richards, J., and Costa, D. L. 1998. Pulmonary responses to oil fly ash particles in the rat differ by virtue of their specific soluble metals. Toxicol. Sci. 43:204-212.

Kodavanti, U. P., Schladweiler, M. C. J., Richards, J. R., and Costa, D. L. 2001. Acute lung injury from intratracheal exposure to fugitive residual oil fly ash and its constituent metals in normo- and spontaneously hypertensive rats. Inhal. Toxicol. 13:37-54.

Lambert, A. L., Dong, W., Selgrade, M. K., and Gilmour, M. I. 2000. Enhanced allergic sensitization by residual oil fly ash particles is mediated by soluble metal constituents. Toxicol. Appl. Pharmacol.165: 84-93. 
Levy, B. S., Hoffman, L., and Gottsegen, S. 1984. Boilmakers' bronchitis: Respiratory tract irritation associated with vanadium pentoxide exposure during oil-to-coal conversion of a power plant. J. Occup. Med. 26:567-570.

Lewis, A. B., Taylor, M. D., Roberts, J. R., Leonard, S. S., Shi, X., and Antonini, J. M. 2003. Role of metal-induced reactive oxygen species generation in lung responses caused by residual oil fly ash. J. Biosci. 28:13-18.

Longphre, M., Li, D., Matovinovic, E., Gallup, M., Samet, J. M., and Basbaum, C. B. 2000. Lung mucin production is stimulated by the air pollutant residual oil fly ash. Toxicol Appl. Pharmacol. 162:86-92.

Nadadur, S. S., Schladweiler, M. C., and Kodavanti, U. P. 2000. A pulmonary rat gene array for screening altered expression profiles in air pollutant-induced lung injury. Inhal. Toxicol. 12:1239-1254.

Nadadur, S. S., and Kodavanti, U. P. 2002. Altered gene expression profiles of rat lung in response to an emission particulate and its metal constituents. J. Toxicol. Environ. Health. 65:1333-1350.

NIOSH. 1994. Elements (ICP): Method 7300. In NIOSH Manual of Analytical Methods, $4^{\text {th }}$ Ed., Issue 2, pp. 1-10. U.S. Department of Health and Human Services, Publication No. 98-119. NIOSH, Washington, DC.

Pope III, C. A., Dockery, D. W., and Schwartz, J. 1995. Review of epidemiological evidence of health effects of particulate air pollution. Inhal. Toxicol. 7:1-18.

Pritchard, R., Ghio, A. J., Lehmann, J. R., Winsett, D.W., Tepper, J. S., Park, P., Gilmour, M. I., Dreher, K. L., and Costa, D. L. 1996. Oxidant generation and lung injury after particulate air pollution exposure increase with the concentration of associated metals. Inhal. Toxicol. 8:457-477.

Rice, T. M., Clarke, R. W., Godleski, J. J., Al-Matairi, E., Jiang, N-F., Hauser, R. and Paulauskis, J. D. 2001. Differential ability of transition metals to induce pulmonary inflammation. Toxicol. Appl. Pharmacol. 177: 46-53

Samet, J. M., Silbajoris, R., Huang, T., and Ilona, J. 2002. Transcription factor activation following exposure of an intact lung preparation to metallic particulate matter. Environ. Health Perspect. 110:985-990.

Schaumann, F., Borm, P. J. A., Herbrich, A., Knoch, J., Pitz, M., Schins, R. P. F., Luettig, B., Hohlfeld, J. M., Heinrich, J., and Krug, N. 2004. Metal-rich ambient particles (particulate matter ${ }_{2.5}$ ) cause airway inflammation in healthy subjects. Am. J. Respir. Crit. Care Med. 170: 898-903. 
Schwartz, J. 1994. What are people dying from on high pollution days? Environ. Res. 64:26-35.

Soukup, J. M., Ghio, A. J., and Becker, S. 2000. Soluble components of Utah Valley particulate pollution alter alveolar macrophage function in vivo and in vitro. Inhal. Toxicol. 12: 401-414.

Tao, F., and Kobzik, L. 2002. Lung macrophage-epithelial cell interactions amplify particle-mediated cytokine release. Am J. Respir. Cell Mol. Biol. 26:449-505.

Van Loveren, H., Rombout, P. J. A., Wagenaar, S. S., Walvoot, H. C., and Vos, J. G. 1988. Effects of ozone on the defense to a respiratory Listeria monocytogenes infection in the rat. Suppression of macrophage function and cellular immunity and aggravation of histopathology in the lung and liver during infection. Toxicol. Appl. Pharmacol. 94:374393.

Zelikoff, J. T., Schermerhorn, K. R., Fang, K., Cohen, M. D., and Schlesinger, R. B. 2002. A role for associated transition metals in the immunotoxicity of inhaled ambient particulate matter. Environ. Health Perspect. 110(Suppl. 5):871-875. 
STUDY 2: ALTERATIONS IN INNATE AND ADAPTIVE PULMONARY IMMUNE RESPONSES TO BACTERIAL INFECTION IN THE LUNGS OF RATS EXPOSED TO THE SOLUBLE METAL FRACTION OF ROFA 


\section{$\underline{\text { Abstract }}$}

Incidences of high levels of air pollution have been correlated with increased morbidity in susceptible populations. The soluble metals of one such pollutant, residual oil fly ash (ROFA), have been shown to alter the clearance of bacteria from the lungs of rats. The goal of this study was to determine the potential effects on both the innate and adaptive immune responses in the lungs of rats exposed to the soluble metals in ROFA prior to pulmonary bacterial infection. ROFA was suspended in phosphate-buffered saline (R-Total), incubated overnight at $37^{\circ} \mathrm{C}$, separated by centrifugation, and the soluble fraction (R-Soluble) was retained. A portion of the soluble sample was treated with the metal-binding resin, Chelex, for $24 \mathrm{hr}$ at $37^{\circ} \mathrm{C}$. Sprague-Dawley rats were intratracheally dosed at day 0 with R-Total $(1.0 \mathrm{mg} / 100 \mathrm{~g}$ body weight), R-Soluble, RSoluble+Chelex (R-Chelex), or phosphate-buffered saline (Saline) (vehicle control). At day $3,5 \times 10^{4}$ Listeria monocytogenes were intratracheally instilled into rats from each treatment group. At days 6, 8 and 10, left lungs were removed, homogenized, and cultured to assess bacterial clearance. Bronchoalveolar lavage (BAL) was performed on the right lungs. A concentrated first fraction of lavage fluid was retained for analysis of lactate dehydrogenase (LDH), albumin, and reactive nitrogen species (RNS) as indices of lung damage, and a variety of cytokines were measured to determine the activity of cells involved in the innate and adaptive immune responses in the lung. BAL cells were collected and cell differentials were performed to assess total number of alveolar macrophages (AMs), neutrophils, and lymphocytes that infiltrated the lungs. In addition, lymphocyte phenotype was analyzed by flow cytometry. Chemiluminescence was used to determine the oxidative potential of AMs and neutrophils, and production of RNS was measured in media collected from BAL cells in culture. Animals treated with R-Total or R-Soluble had significantly more bacteria in the lungs at all time points after infection and a marked increase in lung injury parameters before and after inoculation in comparison to animals treated with R-Chelex or saline. However, only rats pre-treated with R-Soluble had significant increases in AM, neutrophil and lymphocyte infiltration into the lungs post-infection. Total number of lymphocytes was highest at the latest time point and was characterized by increases in CD8+ and CD4+ T cells, as well as natural killer (NK) cells. Oxidant and RNS production was elevated in all groups after infection and there was a significant increase in RNS in the BAL of rats treated with R-Soluble on Day 6 when compared to all other groups. In the R-soluble-treated animals, interleukin (IL)-6 was elevated at the early time points post-infection indicating increased inflammation and a persistent acute phase response. Also, IL-10 was elevated on day 6, possibly leading to an inhibition in or a delayed response in macrophage activity. In the R-Soluble group, IL-18, a cytokine involved in influencing T cell differentiation toward a cell-mediated, rather than humoral, response, was increased on day 8 . There was a significant decrease in IL-12p70 in the R-Total group at the earliest time point after Listeria infection; however, this was not observed in the R-Soluble group. In addition, there were significant decreases in $\mathrm{T}$ cell cytokines in rats pre-exposed to the R-Soluble sample. IL-2 was lower at all time points pre- and post-infection, and IL-4 at the early time points post-infection in the R-Soluble-treated rats. These results suggest that soluble metals present in ROFA increase lung injury and inflammation, alter the ability of the 
phagocytes to clear bacterial infection, and directly or indirectly affect lymphocyte activity in the lung. 


\section{Introduction}

Residual oil fly ash (ROFA) is an air pollutant by-product of the combustion of fossil fuels and contributes significantly to total primary particulate matter (PM) emissions in the U.S. (Environmental Protection Agency, 2004). Increased infectivity and susceptibility to infection have been correlated with high levels of outdoor air pollutants (American Thoracic Society, 1996), resulting in upper and lower respiratory tract infections that interfere with normal activity. Epidemiological and human experimental studies have attributed high metal content of pollutants to increased respiratory inflammation and illness (Ghio, 2004; Leonardi et al., 2000; Schaumann et al., 2004; Soukup et al., 2000). Animal studies have shown that respiratory exposure to ROFA (Antonini et al., 2002; Hatch et al., 1985; Pritchard et al., 1996) or ambient air particulates (Zelikoff et al., 2003) that have a high metal content can increase susceptibility to infection. The previous study demonstrated that it was the soluble metal constituent of ROFA that appeared to suppress the immune response to Listeria monocytogenes instilled in the lungs of rats. The goal of this study was to determine the potential mechanisms by which the soluble metals in ROFA may alter the innate and adaptive immune responses in the lung of rats exposed to the metals prior to pulmonary bacterial infection.

L. monocytogenes has been used by a number of investigators as a model pathogen to investigate respiratory defense mechanisms in response to various environmental exposures (Antonini et al., 2002; Cohen et al., 2001; Jakab, 1993; Steerenberg et al., 2004; van Loveren et al., 1988; Yin et al., 2002; 2003). L. monocytogenes is a gram-positive, facultative, intracellular bacteria that resides in the 
cytosol of infected cells. It can infect a variety of cell types including epithelial cells and phagocytes (Seder and Gazzinelli, 1999). Effective clearance of the bacterial infection requires both the innate (non-specific) and adaptive (pathogen-specific) immune responses. Innate responders include macrophages, neutrophils, dendritic cells (DCs), and natural killer (NK) cells. The primary adaptive immune response to L. monocytogenes is considered to be cell-mediated immunity, consisting of CD8+ T cells and CD4+ $\mathrm{T}_{\mathrm{H}} 1$ cells, rather than humoral immunity, where $\mathrm{B}$ cells and $\mathrm{CD} 4+\mathrm{T}_{\mathrm{H}} 2$ cells would be the responders. Innate responders are the first cells to encounter bacteria or infected cells, and these cells serve two primary functions. The first is to recognize the pathogen or pathogen-infected cells, and to kill and clear the bacteria from the lungs by a variety of bactericidal mechanisms. This process is carried out primarily by alveolar macrophages (AMs), NK cells, and neutrophils. The second function, performed primarily by AMs and DCs, is to process and present the pathogen to the adaptive immune system, and to produce signaling molecules, such as cytokines, that will influence the direction and development of the adaptive response. In response to L. monocytogenes, cell-mediated immunity will result in the recognition and killing of infected cells by CD8+ T lymphocytes and further activation of AMs and NK cells by CD4+ $\mathrm{T}_{\mathrm{H}} 1$ lymphocytes (Pamer, 2004).

ROFA and metals associated with it have been shown to alter the function of the cell types in the lungs as mentioned above. Antonini et al. (2002) showed that exposure to ROFA prior to infection decreased the ability of AMs to kill bacteria in the lung. Investigators have shown that ROFA can alter the oxidative state of AMs (Antonini et al., 2002; Becker et al., 2002; Goldsmith et al., 1999; Lewis et al., 2003) and neutrophils 
(Becker et al., 2002). The ability of phagocytes to produce reactive oxygen and nitrogen species plays an important role in killing of L. monocytogenes; however, excessive oxidative stress and injury may not be beneficial prior to infection. ROFA has also been shown to alter inflammatory cytokine production in the lung by phagocytes and epithelial cells (Gao et al., 2004; Goldsmith et al., 1998; Lambert et al., 2000; Roberts et al., 2003), particularly tumor necrosis factor- $\alpha$ (TNF- $\alpha$ ), interleukin (IL)-1, IL-6, IL-8, macrophage inflammatory protein (MIP)-2 and monocyte chemotactic protein ( MCP)-1, which may affect cell-signaling pathways involved in the immune response. Much of the inflammation and injury observed in response to ROFA has been attributed to the metal content, particularly the soluble metals (Dreher et al., 1997; Dye et al., 1999; Goldsmith et al., 1998; Kodavanti et al., 1998).

A variety of air pollutants have been shown to alter lymphocyte activity, including diesel exhaust (van Zijverden et al., 2000; Yin et al., 2003; 2004a), ambient air PM (Clarke et al., 1999; Leonardie et al., 2000; Omara et al., 1999), and coal fly ash (Dormans et al., 1999). The direct effects of ROFA on lymphocytes, both B and T cells, are not well characterized. Most of the information regarding the function of these cell types in response to ROFA comes from studies that examine the ability of ROFA to enhance sensitization and hyperresponsiveness to allergens in animals (Gavett et al., 1999; Lambert et al., 2000). Gavett et al. (1999) found that ROFA exposure enhanced the activity of $\mathrm{T}_{\mathrm{H}} 2$ cells and increased IL-4 production in ovalbumin-sensitized mice, and Lambert et al. (2000) found that pre-exposure to ROFA, as well as nickel or vanadium (metals present in ROFA), enhanced lymphocyte proliferation in rats sensitized to house dust mites. It has been shown that PM rich in metals that are also present in ROFA can 
have effects on lymphocytes, including alterations in B and T cell proliferation (Zelikoff et al., 2002) and lymphocyte infiltration into the lung (Dye et al., 2001). Therefore, it is possible that ROFA, particularly the soluble metal constituent, may alter the function of lymphocytes involved in the immune response to bacterial infection in the lung.

The ROFA used in this study is a complex mixture of soluble and insoluble metals complexed to a particulate carbon core. The metal compositions of the ROFA, its insoluble fraction, and its soluble fraction were described in the previous study (Table 1.2). Briefly, the soluble constituent contained high levels of nickel, iron, aluminum, and zinc, and was shown to alter bacterial clearance from the lungs of rats. The goal of this study was to determine the alterations in the various immune responses to bacteria in the lung that are associated with the exposure to the soluble fraction of ROFA.

We hypothesized that pre-exposure to the soluble fraction of ROFA prior to bacterial infection would alter the function of a number of cell types involved in the innate and adaptive immune response in the lungs of rats, including phagocytes and lymphocytes. Rats were pre-exposed to ROFA or the soluble fraction of ROFA by intratracheal instillation. Two separate groups of rats were intratracheally instilled with phosphate-buffered saline (vehicle control) or a soluble fraction treated with a metal chelator in order to control for the soluble metal content in ROFA. Following exposure to the ROFA samples, rats were intratracheally inoculated with $L$. monocytogenes at a dose of $5 \times 10^{4}$ bacteria per rat. Infection was characterized by monitoring animal weight, lung injury, and pulmonary clearance of L. monocytogenes. Cellular influx into the lungs of animals pre-exposed to the various ROFA samples, or saline, prior to, and following, infection was monitored, and the number and phenotype of cells were determined. The 
function of cell types involved in the immune response was assessed by measuring production of reactive oxygen and nitrogen species in the lung and the presence of a variety of cytokines in the bronchoalveolar lavage fluid. 


\section{Methods}

Animals - Male Sprague-Dawley [Hla:(SD)CVF] rats (Hilltop Laboratories, Scottdale, PA) weighing 250-300 g, approximately 10 weeks old, were used for all experiments. They were given the ProLab 3500 diet and tap water ad libitum, housed in a clean air and viral- and antigen-free room with restricted access in an AAALACapproved animal facility, and allowed to acclimate for one week before use. The rats were monitored and found to be free of endogenous viral pathogens, parasites, mycoplasms, Helicobacter, and CAR Bacillus.

Materials - L. monocytogenes (strain 10403S, serotype 1) was obtained as a gift from Dr. Rosana Schafer of the Department of Microbiology and Immunology at West Virginia University. Residual oil fly ash (ROFA) was collected from a precipitator at Boston Edison Co., Mystic Power Plant \#4, Everett, MA. The chelating resin, Chelex 100 (iminodiacetic acid), was purchased from Sigma-Aldrich Co., St. Louis, MO.

Experimental Design - At day 0, animals were pre-exposed to ROFA samples or phosphate-buffered saline (vehicle control) by intratracheal instillation. At day 3, the animals were inoculated via intratracheal instillation with $5 \times 10^{4}$ L. monocytogenes. At days 6,8 , and 10, the rats were euthanized, the left lungs of animals were clamped off, removed, homogenized, and the number of colony forming units (CFUs) was counted. The right lungs of animals were lavaged on day 3 (pre-infection), and on days 6, 8, and 10 , and the cells and the fluid were retained for analysis as described bellow. In addition, uninfected animals from each treatment group were sacrificed on days 6, 8, and 10 and monitored for similar parameters as the infected animals. 
ROFA Characterization - Particle size of the ROFA sample was determined by scanning electron microscopy (JSM-\#5600 SEM, JEOL Ltd., Peabody, MA) and previously characterized (Antonini et al., 2002). ROFA particles were of respirable size with a count mean diameter of $2.2 \mu \mathrm{m}$. The metal constituents of the ROFA samples were analyzed using inductively coupled argon plasma, atomic-emission spectroscopy (NIOSH, 1994).

ROFA Treatment - The ROFA sample (R-Total) was suspended in sterile phosphate-buffered saline (PBS) $(6 \mathrm{mg} / \mathrm{ml})$, sonicated for $1 \mathrm{~min}$ with a Sonifier 450 Cell Disruptor (Branson Ultrasonics, Danbury, CT), and allowed to shake and incubate for 24 $\mathrm{hr}$ at $37^{\circ} \mathrm{C}$. The sample was further divided into soluble and insoluble components by centrifugation at $12,000 \mathrm{~g}$ for $30 \mathrm{~min}$. The supernatant of the sample was recovered and filtered (R-Soluble). Chelex was added to the R-Soluble sample (20 mg Chelex/ $0.1 \mathrm{mg}$ ROFA) and incubated on a rotor overnight. The sample was centrifuged and the supernatant of the R-Soluble sample treated with Chelex (R-Chelex) was recovered.

Rats were lightly anesthetized by an intraperitoneal injection of $0.6 \mathrm{ml}$ of a $1 \%$ solution of sodium methohexital (Brevital, Eli Lilly, Indianapolis, IN) and intratracheally instilled with $1.0 \mathrm{mg} / 100 \mathrm{~g}$ body weight (bw) of R-Total ( $\mathrm{n}=8$ )in $300 \mu \mathrm{l}$ of PBS, according to the method of Brain et al. (1976). In addition, R-Soluble ( $\mathrm{n}=8$ ) was administered by intratracheal instillation using the soluble portion equivalent to that in the R-Total instillate. Animals in the control groups ( $\mathrm{n}=8$ per group) were intratracheally dosed with $300 \mu \mathrm{l}$ of sterile PBS (Saline) as a vehicle control or R-Chelex as a control for non-soluble metal constituents in that portion of the R-Soluble sample. The ROFA dose chosen was previously shown to induce inflammation (Antonini et al., 2002), and fell 
within the range of concentrations consistently used in other animal studies evaluating the pulmonary responses to ROFA (Pritchard et al., 1996; Dreher et al., 1997; Gavett et al., 1997; Kodavanti et al., 1998). There were 8-10 animals per group per time point.

Intratracheal Bacteria Inoculation - L. monocytogenes was cultured overnight in brain-heart infusion broth (Difco Laboratories, Detroit, MI) at $37^{\circ} \mathrm{C}$ in a shaking incubator. Following incubation, the bacterial concentration was determined spectrophotometrically at an optical density of $600 \mathrm{~nm}$. The sample was diluted to the concentration of $5 \times 10^{4} \mathrm{~L}$. monocytogenes in $500 \mu \mathrm{l}$ of sterile PBS. The bacteria were intratracheally instilled 3 days post-ROFA sample instillation. The dose of $L$. monocytogenes was chosen based on the first study and was found to give a uniform infection and did not result in mortality in untreated naïve Sprague-Dawley rats in a previous study. Uninfected control animals from each treatment group received saline on day $3(n=4$ per group).

Morbidity/ Pulmonary Clearance of $L$. monocytogenes - Animal weights were monitored over the course of the treatment period as an indicator of morbidity. Rats were euthanized with an overdose of sodium pentobarbital, and at days 6,8 , and 10 , and the left lungs were removed from all the rats in each treatment group. The excised tissues were suspended in $10 \mathrm{ml}$ of sterile water, homogenized using a PowerGen 700 homogenizer (Fisher Scientific, Pittsburgh, PA), and cultured quantitatively on brainheart infusion agar plates (Becton Dickinson and Co., Cockeysville, MD). The number of viable CFUs was counted after an overnight incubation at $37^{\circ} \mathrm{C}$.

Bronchoalveolar Lavage (BAL) - BAL was performed by washing out the lungs of the rats with aliquots of PBS in order to obtain pulmonary cells for morphological and 
functional analysis, and the acellular BAL fluid was retained for analysis of indicators of tissue damage and cellular activity. Rats were euthanized with an overdose of sodium pentobarbital, the left lungs were clamped off and removed, and BAL was performed on the right lungs on day 3 prior to infection, and at days 6,8 , and 10. The first BAL volume was administered as $1 \mathrm{ml}$ of PBS per 100g body weight. The volume remained in the lungs for 30 seconds with light massaging, was withdrawn, and re-instilled for another 30 seconds. This concentrated aliquot was withdrawn, retained, kept separately, and was designated as the first fraction of BAL fluid. The following aliquots were $6 \mathrm{ml}$ in volume, instilled once with light massaging, withdrawn, and combined. The procedure was performed until a $30 \mathrm{ml}$ volume was obtained. For each animal, both fractions of BAL were centrifuged, the cell pellets were combined and resuspended in $1 \mathrm{ml}$ of PBS, and the acellular fluid from the first fraction was retained for further analysis.

Analysis of Albumin and Lactate Dehydrogenase (LDH) - The presence of albumin and LDH in the BAL fluid was measured to evaluate the loss of integrity of the alveolar-capillary barrier and general cytotoxicity, respectively. Measurements of both albumin and LDH in the acellular fluid were obtained using a Cobas Mira analyzer (Roche Diagnostic Systems, Montclair, IN). Albumin was determined by spectrophotometric measurement of the reaction product of albumin with bromcresol green $(628 \mathrm{~nm})$ according to a method by Sigma Diagnostics (St. Louis, MO). LDH activity was quantified by detection of the oxidation of lactate coupled to the reduction of $\mathrm{NAD+}$ at a spectrophotometric setting of 340nm over time.

Pulmonary Cell Differentials and Phenotyping - Total BAL cells were counted using a Coulter Multisizer II (Coulter Electronics, Hialeah, FL). Cell differentials were 
performed to determine the total number of alveolar macrophages (AMs), polymorphonuclear cells (PMNs), and lymphocytes. Briefly, $1 \mathrm{x} 10^{5}$ cells were adhered onto slides using a cytospin (Shandon Inc., Pittsburgh, PA), stained with a WrightGiemsa stain (HEMA 3, Fisher Scientific, Pittsburgh, PA), and a total of 200 cells per slide were counted. The percentage of AMs, PMNs, and lymphocytes were multiplied across the total number of cells to calculate the number of each cell type. To determine phenotype of the lymphocyte population in the BAL, cells were labeled with fluorescently tagged antibodies against specific cell surface markers (BD Biosciences Pharmingen, San Diego, CA). The lymphocytes that were identified were NK cells $\left(\mathrm{CD} 161 \mathrm{a}^{+}, \mathrm{CD} 3^{-}\right)$, B lymphocytes $\left(\mathrm{CD} 45 \mathrm{R}^{+}\right)$, and $\mathrm{T}$ lymphocytes $\left(\mathrm{CD} 3^{+}\right)$. The $\mathrm{T}$ cells were further differentiated as T-helper $\left(\mathrm{T}_{\mathrm{H}}\right)$ cells $\left(\mathrm{CD}^{+}\right)$or cytotoxic $\mathrm{T}$ cells $\left(\mathrm{CD} 8^{+}\right)$. Using a flow cytometer (FACS Calibur, BD Biosource, San Diego, CA), the lymphocyte population of the BAL cells to be analyzed was determined by size using forward and side scattering, and the viable cells were selected by eliminating the population of dead cells that had stained positive for 7-amino-actinomycin D (7-AAD). Percentages of each cell type in BAL measured with flow cytometry were calculated and multiplied back across the total number of lymphocytes determined from the cell differentials.

Measurement of Nitric Oxide - The presence of nitric oxide in acellular BAL was determined as the accumulation of nitrite using a modified microplate assay using the Greiss reagent (Green et al., 1982). Briefly, total nitrite was measured after a 1 hour incubation with a nitrate reductase to convert any nitrate in the sample to nitrite. The microplate was then centrifuged, and the supernatant was transferred to another microplate to stop the enzymatic reaction and incubated with the Greiss reagent 
(Molecular Probes, Eugene, OR) for 10 minutes. The absorbance of the samples was analyzed on a SPECTRAmax TM 250 spectrophotometer (Molecular Devices Co., Sunnydale, CA) at 550nm. The measurement of total nitrite represents the presence of both nitrate and nitrite (NOx) in the sample. In addition, total nitrite production by BAL cells was also measured. BAL cells were cultured overnight in endotoxin-free Eagle's minimum essential medium (EMEM) (BioWhittaker, Walkersville, MD) supplemented with $10 \%$ fetal bovine serum, $2 \mathrm{mM}$ glutamine, and penicillin/streptomycin (100 units/ml) (BioWhittaker, Walkersville, MD) in 24-well culture plates at a concentration of $1 \times 10^{6}$ cells per well. After an18-hour incubation, cells were centrifuged out of the media, and the media was retained for the assay described above.

Luminol-Dependent Chemiluminescence (CL) - Luminol-dependent CL was performed on BAL cells as a measure of the light generated by the production of reactive oxygen species (ROS) by AMs and PMNs using a Berthold LB953 luminometer (Wallace Inc., Gaithersburg, MD) as described previously (Antonini et al., 1994). Phorbol 12-myristate 13-acetate (PMA) $(10 \mu \mathrm{M})$, a soluble stimulant of total BAL phagocytes (AMs and PMNs), or non-opsonized, insoluble zymosan ( $2 \mathrm{mg} / \mathrm{ml})$, a stimulant of AMs only (Castranova et al., 1990), was added to the assay immediately prior to CL measurement to determine the contribution of both AMs and PMNs to the overall production of ROS in the lungs of the rats. Measurement of CL was recorded for 15 minutes at $37^{\circ} \mathrm{C}$, and the integral of counts per minute (cpm) versus time was calculated. CL was calculated as the cpm of the stimulated cells minus the cpm of the corresponding resting cells. 
Cytokine Measurements in BAL - Cytokines present in the BAL fluid were analyzed by enzyme-linked immunosorbent assay (ELISA) using commercially available ELISA kits (BioSource International Inc., Camarillo, CA) to determine activity of various cell types involved in the immune response. The following cytokines were quantified: tumor necrosis factor- $\alpha$ (TNF- $\alpha$ ), interleukin (IL)-2, IL-4, IL-6, IL-10, IL-12p70, IL-18, and interferon- $\gamma(\mathrm{IFN}-\gamma)$.

Statistical Analysis - Results are expressed as means \pm standard error of measurement (SE). Statistical analyses were carried out with the SigmaStat 3.1 statistical program (Chicago, IL). The significance of the interaction among different treatment groups for the different parameters at each time point was assessed using analysis of variance (ANOVA). The significance of difference between individual groups was analyzed using the Student-Newman-Keuls method with the criterion of significance set at $p<0.05$. 


\section{$\underline{\text { Results }}$}

ROFA characterization - The ROFA used in this study had been previously characterized (Antonini et al., 2002) and metal content was presented in study 1 (Study 1, Table 2). Briefly, the R-Total sample consisted primarily of iron, nickel, aluminum, calcium, vanadium, and zinc. The R-Soluble sample was comprised of nickel, iron, aluminum, and zinc, but contained very little vanadium. The chelator, Chelex, successfully removed the majority of metals from the R-Soluble sample leaving only a small amount of iron and less than $1 \mu \mathrm{g}$ of vanadium.

Morbidity and Pulmonary Bacterial Clearance - Morbidity in animals pretreated with Saline, R-Total, R-Soluble, or R-Chelex was monitored as the \% change in body weight after infection with L. monocytogenes (Figure 2.1A). Rats pretreated with RTotal or R-Soluble lost significantly more weight post-infection when compared to controls. Animals in the R-Chelex group did not differ from the saline group. Rats in all of the treatment groups began to regain bodyweight by day 8 , although animals in the RTotal and R-Soluble group weighed significantly less at all time points post infection. Uninfected animals from all treatment groups gained weight throughout the course of the study (Study 1, Table 1.3). Animals treated with R-Chelex or Saline were able to clear a large portion of bacteria from the lungs by day 10 (Figure 2.1B), whereas animals preexposed to R-Total or R-Soluble had a significantly greater bacterial lung burden at the early time points post-infection. The significant increase in CFUs in the lungs of the RSoluble group persisted throughout the time course, which was comparable to the findings in study 1 . There was no decrease in survival at this dose of bacteria and animals in all groups had begun to recover by the end of the time course. 
Biochemical Analysis of BAL Fluid: LDH and Albumin- The presence of the intracellular enzyme, $\mathrm{LDH}$, in the BAL fluid of rats was used a marker for general cellular cytotoxicity. LDH was elevated in the uninfected R-Total and R-Soluble group on day 3 (Figure 2.2A). The increase persisted in the uninfected R-Total rats on day 6 when compared to all other groups and on day 8 when compared to saline and R-Chelex controls (data not shown). There were significant elevations in LDH in the uninfected RSoluble-treated rats at all time points when compared to both the Saline and R-Chelex groups (data not shown). After infection, animals pre-treated with R-Soluble had significantly higher LDH levels on days 6 and 8 when compared to control animals (Figure 2.2A), which were slightly higher than levels in the uninfected R-Soluble group. Also, LDH was increased in the infected R-Total animals on day 6 when compared to controls. The R-Chelex group did not differ from control. Albumin in the BAL fluid, an indicator of the breakdown of the alveolar blood-air barrier, was increased in the R-Total and R-Soluble groups on day 3 pre-infection as compared to controls (Figure 2.2B). The increase in albumin persisted in the uninfected R-Total group up to day 6, and in the uninfected R-Soluble group, albumin levels were elevated when compared to all groups throughout the time course (data not shown). Albumin further increased and remained elevated in the R-Soluble group at all time points post-infection when compared to all groups (Figure 2.2B).

BAL Cellular Profiles - Total BAL cell number (Figure 2.3A) was counted, and the total number of AMs (Figure 2.3B), PMNs (Figure 2.3C), and lymphocytes (Figure 2.3D) were calculated from cell differentials. On day 3, prior to infection, rats preexposed to R-Soluble and R-Total had a significantly greater number of PMNs and 
lymphocytes when compared to the Saline and R-Chelex groups, indicating an enhanced inflammatory response in these groups. The increase in PMNs and lymphocytes in the uninfected R-Soluble and R-Total group persists out to day 10, decreasing slightly from day 3 totals over the time course (data not shown). In general, in response to the infection, there is a notable increase in the number of cells in the airspace in all groups post-inoculation with L. monocytogenes. However, the animals treated with R-Soluble had a significantly increased number of total BAL cells when compared to all other groups post-infection. The increase can be attributed to the influx of PMNs at the early time points post-infection (Figure 2.3C), and to the macrophage and lymphocytes populations at the later time points (Figures $2.3 \mathrm{~B}+\mathrm{D}$ ).

Flow cytometry, in conjunction with cell differential counts, was used to determine total number of NK cells (Figure 2.4A), total T cells (Figure 2.4B), CD4+ T cells (Figure 2.4C), and CD8+ T cells (Figure 2.4D). Prior to infection, rats pre-exposed to R-Total showed a slight increase in the number of NK cells when compared to saline control, and rats pretreated with R-Soluble showed a slight increase in the number of $\mathrm{T}$ cells, although the latter was not statistically significant when compared to control. At the early time points post-infection, the R-Soluble group tended to have lower numbers of NK cells when compared to the saline group, although the difference was not significant. However, on day 10 there was a significant increase in the total NK cell number when compared to all groups (Figure 2.4A). There were no statistically significant changes in the number of $\mathrm{T}$ lymphocytes until day 10 at which time there was a significant increase in the total number of T-cells in the R-Soluble group when compared to all other groups. 
This was attributed to both subsets of the T cell population, CD4+ (Figure 2.4C) and CD8+ (Figure 2.4D) T cells.

On day 3 , before infection, there were no significant differences among the groups in the T/B cell ratio (Table 2.1) or the $\mathrm{CD} 4+/ \mathrm{CD} 8+\mathrm{T}$ cell ratio (Table 2.2). There was a general increase in the B cell population early after infection on day 6 , leading to the initial drop in the T/B cell ratio. However, over the course of the infection, there was a continual increase in this ratio reflecting the dominance of the cell-mediated immune system in response to L. monocytogenes. On day 8 , there was a significant decrease in the number of B cells in the R-Soluble group when compared to the saline group reflected as a significantly higher T/B cell ratio at this time point. Post-infection, there was an overall decrease in the $\mathrm{CD} 4+/ \mathrm{CD} 8+\mathrm{T}$ cell ratio in all groups post-infection. Although both $\mathrm{T}$ cell subpopulations increased after bacterial infection, the decreasing ratio was due primarily to a greater increase in the $\mathrm{CD} 8+$ cell population which was much lower prior to infection.

BAL Fluid Levels and BAL Cellular Production of Nitric Oxide - The presence of nitric oxide, a known antimicrobial agent, as well as an indicator for increased oxidative stress, was measured as total nitrite (nitrate and nitrite, NOx) (Figure 2.5). NOx in the BAL fluid was significantly elevated in the R-Soluble group when compared to all other groups at the early time point post-infection (Figure 2.5A). NOx production by BAL cells did not differ among treatment groups on day 3 prior to infection (Figure 2.5B) and did not differ among uninfected animals in the various treatment groups at other times (data not shown). Post-infection, NOx production by cells in all treatment groups (measured from cell culture media) increased markedly when 
compared to levels on day 3; however, BAL cells from the R-Soluble and R-Total groups produced significantly less NOx than the control groups on day 6 (Figure 2.5B). On day 8, BAL cells from the R-Soluble group continued to produce less total nitrite when compared to the saline-treated animals. By day 10, NOx production remained elevated for all treatments and there were no significant differences among groups.

\section{Oxidative Potential of Phagocytes: PMA- and zymosan-stimulated CL -}

Luminol-dependent CL was performed on BAL cells to determine the level of production of reactive oxygen species (ROS) by AMs and PMNs. There were no significant differences among treatment groups prior to infection after stimulation with PMA (Figure 2.6A) or zymosan (Figure 2.6B), and there were no significant differences in uninfected animals on days 6,8 or 10 after exposure to the various ROFA samples (data not shown). After infection, on day 6, there was an increase in both PMA- and zymosan-stimulated $\mathrm{CL}$ in all of the treatment groups; however, the increase in the R-Total-treated rats was not as great when compared to all other groups. In addition, there was a slight, but significant, increase in oxidant production by both stimulants in the R-Soluble and RChelex groups on day 8 when compared to the Saline control. By day 10, oxidant production had returned to levels observed prior to infection. Overall, the pattern and the levels of oxidant production seen with zymosan stimulation were comparable to that observed with PMA stimulation for all groups at all time points, indicating that the primary oxidant producer in response to the infection was the AM. However, because both R-Total and R-Soluble groups had a higher bacterial burden on day 6 than the controls, and only the R-Total-treated rats showed a decrease in oxidative potential on 
day 6 , it is not likely that the ROS production, or lack there of, is the primary reason for the alteration in the course of the infection due to the soluble metal component of ROFA.

BAL Cytokine Analysis - Cytokines present in the BAL were measured as indicators of cell-specific activity in response to the ROFA samples and to the $L$. monocytogenes infection. TNF- $\alpha$ (Figure 2.7A) and IL-6 (Figure 2.7B) were measured in the BAL as indicators of the degree of the inflammatory response. On day 6 , there was an increase in TNF- $\alpha$ in the BAL in all the groups as a response to the bacterial infection; however, there were no significant differences among treatments at day 3 , prior to infection, or at any of the time points post-infection (Figure 2.7A). Another inflammatory cytokine, IL-6, is indicative of the acute phase response, the reaction following insult or injury that results in fever, an increase in circulating leukocytes, particularly neutrophils, and an increase in phagocyte activity (Hirano et al., 1990). On day 3, pre-infection, a significant increase was observed in the BAL level of IL-6 in the R-Total group when compared to all other groups (Figure 2.7B). Post-infection, on days 6 and 8 , there was a significant elevation in IL-6 in the animals pre-treated with R-Soluble when compared to all other treatments, indicating a more persistent and severe inflammatory response.

IL-10 has diverse effects on different cell types, but is considered to primarily be inhibitory in nature, and to be an anti-inflammatory cytokine. It is known to inhibit activation of CD4+ $\mathrm{T}_{\mathrm{H}} 1$ and $\mathrm{CD} 8+\mathrm{T}$ cells, monocytes, macrophages and neutrophils, and have varying effects on NK cells depending on the presence of other cytokines (Moore et al., 2001). These cell functions are necessary in the cell-mediated immune response $L$.

monocytogenes. At day 6, IL-10 levels in the BAL of animals pre-treated with R-Soluble 
remained elevated when compared to the Saline control rats, potentially inhibiting cells involved in the clearance of the bacteria from the lungs (Figure 2.8).

IL-12 and IL-18 are two cytokines known to induce the expression of IFN- $\gamma$ by $\mathrm{CD} 4+\mathrm{T}_{\mathrm{H}} 1$ cells, $\mathrm{CD} 8+\mathrm{T}$ cells, and NK cells, and the presence of IL-12 and IFN- $\gamma$, in turn, drives the differentiation of naïve CD4+ T cells toward the CD4+ $\mathrm{T}_{\mathrm{H}} 1$ subset (Billiau, 1996; Mosmann and Sad, 1996; Paul and Seder, 1994). There were no significant differences among groups in the levels of IL-12p70 (the active form of IL-12) (Figure 2.9A) or IL-18 (Figure 2.9B) prior to infection. The highest levels of IL-12, secreted primarily by innate responders, were observed at the early time point postinfection; however, the R-Total group, but not the R-Soluble group, produced significantly less IL-12 than all other groups. IL-12 should have a greater impact on the immune response in the early stages of infection as opposed to the later phase. Therefore, the significant decrease in IL-12 at day 10 in the R-Soluble group when compared to controls likely did not affect the course of the infection. IL-18 increased in all groups post-infection and was significantly elevated in the R-Soluble rats on day 8 when compared to controls.

To determine the potential activity of the $\mathrm{CD} 4+\mathrm{T}$ cell subsets in the lung, levels of IL-2 and IL-4 were measured in the BAL fluid. IL-2 is produced primarily by CD4+ $\mathrm{T}_{\mathrm{H}} 1$ cells and by naïve $\mathrm{CD} 4+$ and some $\mathrm{CD} 8+\mathrm{T}$ cells, whereas IL-4 is secreted mainly by the CD4 $+\mathrm{T}_{\mathrm{H}} 2$ subset (Mosmann and Sad, 1996). Levels of IL-2 were significantly reduced prior to infection on day 3 in the R-Soluble group when compared to controls, and at all time points post-infection, when compared to all other treatment groups (Figure 2.10A). After infection, there was significantly less IL-4 in the BAL of rats pre-treated 
with R-Soluble when compared to controls on day 6 (Figure 2.10B). The trend for the decrease in IL-4 in the R-Soluble group continued on days 8 and 10; however it was not statistically significant at those time points. Changes in the levels of these cytokines may be indicative of alterations in $\mathrm{T}$ cell function due to soluble metals.

IFN- $\gamma$ is also produced by $\mathrm{CD} 4+\mathrm{T}_{\mathrm{H}} 1$ cells, as well as by $\mathrm{CD} 8+\mathrm{T}$ cells and $\mathrm{NK}$ cells. There were no significant differences prior to infection among groups, and there was a general increase at the first time point post-infection. However, levels of this cytokine in the BAL were extremely variable across all time points, as well as within groups at a single time point (data not shown). Production of IFN- $\gamma$ is stimulated by TNF$\alpha$ and IL-1 $\beta$ and is an early response in the course of the infection. Differences in IFN- $\gamma$ levels may be better detected at an earlier time point post-inoculation. Furthermore, in future experiments it may prove more effective to measure levels of IFN- $\gamma$ as it is produced by lymphocytes cultured directly from the tracheobronchial lymph nodes of the treated animals or in the tissue of the lung, rather than in the acellular BAL fluid.

There were no notable differences in BAL cytokine levels in the uninfected treatment groups on days 6,8 , or 10 post-ROFA exposure (data not shown). 


\section{$\underline{\text { Discussion }}$}

The goal of this study was to determine the potential effects on both the innate and adaptive immune responses in the lung of rats exposed to the soluble metals in ROFA prior to pulmonary bacterial infection. This study found that the soluble metals in ROFA caused increased lung injury and cytoxicity prior to infection, which is in agreement with the findings of other investigators (Dreher et al., 1997; Dye et al., 1999;

Kodavanti et al., 1998), and that the increased injury and cytotoxicity persisted throughout the time course post-infection. In addition, this study confirmed the results of the study 1 , demonstrating that the soluble fraction of ROFA decreased bacterial clearance from the lungs of rats to a comparable, if not greater degree, when compared to that of the whole ROFA sample. The soluble sample caused a greater influx of neutrophils into the lungs at the early time points post-infection, and macrophages and lymphocytes at the later time points, and appeared to alter the activity of macrophages and lymphocytes (CD4+ T cells, and CD8+ T cells) post-infection. In addition, the soluble sample altered production of reactive nitrogen species, IL-6, IL-10, IL-18, IL-2 and IL-4 in the lungs of rats post-infection. This finding indicates that the soluble metals may adversely affect phagocyte and lymphocyte function, consequently altering the innate and adaptive immune response to L. monocytogenes infection. These effects were abolished when the metals were removed from the soluble sample by Chelex, confirming that the soluble metals were responsible for the results, rather than the non-metal constituents present in the sample.

At 3 days post-ROFA exposure, and prior to infection, the primary differences that existed among the animals exposed to the soluble metals or the total ROFA when 
compared with control groups were increases in LDH and albumin, indicators of cytotoxicity and lung injury. There was also a smaller, but significant, increase in neutrophils and lymphocytes, indicative of increased inflammation; however, overall cell number in the BAL did not differ. The increased injury and inflammation that was observed early after exposure with the soluble fraction of ROFA was consistent with the findings of other investigators (Dreher et al., 1997; Kodavanti et al., 1998). Similar indicators of injury and inflammation are also observed with the soluble metal fractions of Utah Valley Dust (UVD) (Dye et al., 2001) and welding fumes (Antonini et al., 2004). It is possible that the soluble metals, which continued to cause inflammation and cytotoxicity directly in the lungs of uninfected rats at the later time points, further added to the inflammatory response and injury caused by the infection itself. The excessive inflammation and injury after infection (indicated by increased neutrophils, IL-6, LDH, and albumin) that may have resulted from both the direct and indirect effects of the metals may have compromised the host cells' ability to resist infection. Consequently, this response may have contributed to the inability of the animals pretreated with the soluble metals to clear the bacteria as effectively as the control rats. Despite the injury and inflammation, baseline cellular activity by the innate responders, such as macrophages, neutrophils, and NK cells, does not appear to be altered prior to bacterial challenge. By 3 days post-exposure to the ROFA samples, cellular production of nitrogen and oxygen radicals did not differ significantly among groups, and the only increase in inflammatory cytokines appeared in the total ROFA group with a slight elevation in IL-6. Interestingly, although $\mathrm{T}$ cell populations in the lung did not differ significantly among groups prior to infection, there was a decreased level of IL-2 in the soluble group when 
compared to controls, indicating that the soluble metals may be directly inhibiting $\mathrm{T}$ cells. Because the cell-mediated immune response is critical in L. monocytogenes infection, it is possible that if the soluble metals persist in the lung throughout the time course, direct effects of these metals may be responsible for alterations in the later adaptive cellular responses to the bacteria.

The initial, innate immune response to L. monocytogenes infection involves recruitment of neutrophils, macrophages, monocytes, and NK cells to the infected area. It is possible that the responsiveness of these cells was altered or inhibited as suggested by the findings of Antonini et al. (2002) which showed that macrophage bactericidal activity in response to Listeria challenge was suppressed in animals that were pre-treated with ROFA prior to infection. This study indicates that the soluble metals may be the fraction of the ROFA that is responsible for impaired innate immune response. Another potential contributor to the early increase in bacterial burden may be the level of injury induced in the lung by the soluble metals themselves. Cytokine levels in the BAL and cellular production of reactive species were assessed to try to determine the activity levels of the innate responders, as well as the direction of the overall immune response.

The pro-inflammatory cytokine cascade, which includes TNF- $\alpha$, IL-6, and IL-12, is a critical step in the innate immune response to L. monocytogenes and leads to a progression that results in initiation of cell mediated immunity and further activation of innate responders (Seder and Gazzinelli, 1999). All three of these cytokines were expected to increase post-infection in all groups. However, based on the degree of inflammation and the increase in bacterial burden in the lungs of the animals exposed to soluble metals prior to infection, a more significant elevation in TNF- $\alpha$ was expected. 
Because there was a significant increase in IL-6 in the R-Soluble-treated group on day 6, it is likely that TNF- $\alpha$, an inducer of IL-6, was elevated in that group at an earlier timepoint post-infection.

IL-6 is a pleiotropic cytokine that is produced by many cell types resulting in a variety of effects. IL-6 plays an important role in the acute-phase response, inflammatory response, and, in conjunction with IL-1, IL-6 has been shown to be involved in the initiation of cytotoxic T cell (CD8+) responses (Akira et al., 1990; Ford et al., 1991; Lotz et al., 1988; Renauld et al., 1989). In addition, IL-6 may play a critical role in the neutrophil response to L. monocytogenes as well. Animals that are deficient in IL-6 have a low neutrophil recruitment and do not survive infection (Dalrymple et al., 1995; Kopf et al., 1994). IL-6 increased in all groups following infection; however, there was a dramatic elevation in IL-6 on day 6 and 8 in the soluble group when compared to all other groups, which corresponded well with the significant influx of neutrophils early on, and cytotoxic T cells (CD8+) later, into the lungs of rats that received the soluble metals prior to infection. The elevation in IL-6 may be indicative of the increased lung injury and an exacerbated acute-phase response due to a delay in, or the suppression of, the early innate immune response that led to the increased bacterial burden on day 6 .

The pro-inflammatory cytokines, including TNF- $\alpha$, IL-12, and IL-6, as well as IL-18, are also known to induce production of reactive oxygen and nitrogen species in macrophages and neutrophils, either directly or through the induction of IFN- $\gamma$ production (Billiau, 1996; Borish et al., 1989; Neighbors et al., 2001; Swain, 2001). Reactive oxygen and nitrogen species contribute to intracellular bacterial killing (MacMicking et al., 1995; Nacy et al., 1991; Shiloh et al., 1999), and NO has been shown 
to play a regulatory role in cell-mediated immunity. Inducible nitric oxide synthase (iNOS) knock-out mice have an excessive $\mathrm{T}_{\mathrm{H}} 1$ response to infection (MacLean et al., 1998); and elevated levels of NO can result in inhibition of T cell proliferation (Blesson et al., 2002, Hoffman et al., 2002; van der Veen et al., 1999;2000) and reduction in IL-2 production (Blesson et al., 2002).

There was an increase in oxidant production, primarily by AMs, in all groups at the early time point post-infection; however, the increase in the total ROFA sample was significantly less when compared to all other groups. Although the total ROFA sample appeared to inhibit ROS production by macrophages, which may contribute to a decrease in bacterial killing in that group, the response was not replicated with the soluble fraction. Previous studies showed that the particulate portion of ROFA did not alter bacterial clearance. Therefore, this effect may be attributable to total metal content or an alteration in metal distribution due to the presence of the insoluble fraction in the total sample, and does not likely contribute to the decrease in clearance of bacteria observed in the soluble sample. Also, CL data was measured on a per cell basis. When considering that the total number of pahogocytes in the lungs of the R-Soluble-treated group was significantly elevated at all time points post-infection, it is likely that the total lung oxidant burden was elevated in this group. This may contribute to an increased oxidative stress and lung injury in thes animals and further comprise their ability to clear the bacteria.

Treatment with the soluble fraction of ROFA did result in a substantial increase in nitric oxide in the BAL post-infection when compared to all other groups, including the total sample. At day 6, where the most significant increase in BAL NOx was observed, there were significantly more neutrophils infiltrating the lungs of the rats exposed to the 
soluble metals. An increase in the AM number in the soluble group was not observed until the later time points post-infection. Therefore, neutrophils may be contributing to the increase in NOx, or the AMs or monocytes present may have increased iNOS activity, or a combination of both. Although NO is involved in anti-microbial activities of phagocytes, excessive elevation in NO may be detrimental to the cell-mediated immune response by inhibition of proliferation and activity of both CD4+ and CD8+ T-cells (Blesson et al., 2002, Hoffman et al., 2002; van der Veen et al., 1999; 2000). Kradin et al. (1999) found that elimination of the AMs from the lungs or rats, which in turn significantly reduced the amount of $\mathrm{NO}$ in the lungs, resulted in increased $\mathrm{T}_{\mathrm{H}} 1$ activity and promoted pulmonary cell-mediated immunity to heat-killed Listeria (HKL). The significant elevations in NO in response to R-Soluble after infection may contribute to the blunted $\mathrm{T}$ cell responses that were observed in this group, as indicated by decreased levels of $\mathrm{T}$ cell cytokines, which are discussed later.

Interestingly, when NOx production was measured on a per cell basis, there was a significant decrease in the NOx levels in the total and soluble groups at the corresponding time point after infection. It is important to note that the ratio of neutrophils to AMs differed in the R-Soluble group ond ay 6; however, if a correction is made for the number of AMs in the R-Soluble-treated rats at that time point (approximately $25 \%$ ) there is still a significant decrease in the cellular production of NOx. Also, there was no difference in the ratio of cells for the total group at this time point, but there was still a significant decrease in NOx production by the BAL cells. This suggests that the increase in NOx in the BAL of the soluble group may be a product of the neutrophils rather than AMs and NOx production by AMs may be inhibited in response to the soluble metals of ROFA. 
These data are in agreement with the findings of Antonini et al. (2002) where at the early time point after infection, animals pre-treated with the total ROFA showed a decrease in NOx production by AMs. The authors also showed that AMs from animals exposed to ROFA prior to infection had decreased intracellular killing of bacteria, a mechanism that has been attributed to the ability of AMs to produce nitric oxide (MacMicking et al., 1995; Green et al., 1994; North et al., 1997).

AMs are critical in the killing and clearance of L. monocytogenes. In fact, suppression of AM activity in the lungs has been shown to be correlated with decreased clearance of $L$. monocytogenes in response to the whole ROFA sample (Antonini et al., 2002) and to other air pollutants, such as diesel (Yang et al., 2001; Yin et al., 2002; 2004b) and ozone (van Loveren et al., 1988). Alterations in IL-10 production may be involved in the inhibition of AM killing of L. monocytogenes. IL-10 is an antiinflammatory cytokine produced by a variety of cell types including activated macrophages, and has effects on a number of different cells including NK cells, DCs, T cells, B cells, monocytes, neutrophils, and, notably, macrophages (Moore et al., 2001). IL-10 inhibits activation of macrophages by NK cells and $T_{H} 1$ cells by blocking INF- $\gamma$ and TNF- $\alpha$ signals from these cells, which in turn activate intracellular killing of pathogens by the AMs. It also directly inhibits macrophage production of cytokines that drive the cell-mediated immune response. In addition, L. monocytogenes, and other intracellular pathogens that specifically target macrophages for infection, use IL-10 to prolong survival by suppressing the host immune response (Redpath et al., 2001).

The current study found that IL-10 was significantly elevated on day 6 in the rats treated with soluble metals prior to infection, and that this increase correlated with the 
highest bacterial burden in the lungs of these animals. Similar results have been observed in other studies that utilize the respiratory L. monocytogenes model of infectivity. Yin et al. (2004a and 2004b) found that pre-exposure to diesel led to a marked increase in IL-10 production by macrophages and increased lung bacterial burden after infection. Similarly, Antonini et al. (2004) found that IL-10 increased after infection in rats pre-exposed to welding fume, and that bacterial clearance decreased. Interestingly, the factor that appeared to influence bacterial clearance by rats pre-treated with different welding fumes in the study was the metal solubility of the fume, with the most soluble fume having the greatest impact on bacterial clearance.

As mentioned previously, IL-10 can alter production of inflammatory cytokines that are also involved in the initiation of a cell-mediated immune response, such as IL12p70, IL-18, and IFN- $\gamma$. IL12 and IL18, produced by antigen-presenting cells, including macrophages and DCs, are highly involved in directing the development of the $\mathrm{T}_{\mathrm{H}} 1$ subset of CD4+ T cells (Hsieh et al., 1993; Stoll et al., 1998; Swain, 2001; Trinchieri, 1995), and directing IFN- $\gamma$ production by NK cells (Chan et al, 1992; Naume et al., 1993; Okamura et al., 1995; Tsutsui et al., 1996;) and CD8+ T cells (Berg et al., 2002), both of which result in further bactericidal activity by macrophages. IL-18 has also been shown to stimulate macrophages directly to produce TNF- $\alpha$ and NO, which would result in increased bacterial killing (Neighbors et al., 2001). Reduction in IL-12 (Tripp et al., 1993; 1994) and IL-18 (Berg et al., 2002; Neighbors et al., 2001) results in a more severe L. monocytogenes infection in rodents. Discrepancies between R-Soluble-treated rats and R-Total-treated animals made it difficult to distinguish how ROFA may be altering antigen-presentation and $\mathrm{T}$ cell differentiation. However, there was a significant increase 
in IL-18 in the R-Soluble group when compared to all other groups. One explanation for this may be that, although macrophage function and bacterial clearance appeared to be inhibited by the soluble metals, DC cytokine production and antigen-presentation may be unaffected; therefore, there would be little or no reduction in the $\mathrm{T}$ cell infiltration into the lungs of rats pre-treated with soluble metals prior to infection. In fact, by day 8 and 10 , there was a significant increase in the lymphocyte response in the soluble metals group when compared to all other groups. Future experiments that examine lymphocyte production of cytokines harvested from the lung-draining lymph nodes may better explain the direction of the early lymphocyte response in these animals.

Activation of the cell-mediated arm of the immune system is critical in long term elimination of L. monocytogenes infection (Bancroft et al., 1991; Emmerling et al., 1975; Nickol and Bonventre, 1977). The adaptive immune response to the infection by all groups included an increase in macrophage and lymphocyte influx into the lung over the time course. The response included increases in NK cells, and CD4+ and CD8+ T cells. The ratio of $\mathrm{CD} 4+/ \mathrm{CD} 8+\mathrm{T}$ cells (Table 2.2) decreased after infection and remained decreased at day 10 indicating a greater population of CD8+, cytotoxic, T cells, as would be expected in response to infection with an intracellular bacteria residing in the cytoplasm. As mentioned previously, the adaptive responders to the infection in the rats that received the soluble ROFA metals were increased on day 10 when compared to all other groups, including that of the total ROFA, with significant increases in CD4+ and CD8+ T lymphocytes, as well as NK cells and macrophages. Rats in the soluble metals group may have required a stronger $\mathrm{T}$ cell response, as well as an increase in 
macrophages and NK cells, in order to kill and clear the increased bacterial burden that persisted through day 10 .

The activity of the $\mathrm{T}$ cells that responded to the infection may provide evidence as to why the higher bacterial burdens persisted in the soluble metals group throughout the time course of the infection. Animals treated with R-Soluble prior to infection had significantly reduced levels of IL-4, a CD4+ $\mathrm{T}_{\mathrm{H}} 2$ cytokine, and more importantly lower IL-2, a cytokine produce by undifferentiated T cells, as well as CD4+ $\mathrm{T}_{\mathrm{H}} 1$ and CD8+ T cells (Janeway et al., 2001). Although $\mathrm{T}_{\mathrm{H}} 2$ cells do not play a major role in the response to L. monocytogenes, it has been suggested that an initial burst in IL-4 within the first day of infection aids in recruitment of neutrophils and monocytes to the area of infection by stimulating chemokine production (Kaufmann et al., 1997). IL-2 plays an important role throughout the course of the infection, in an autocrine fashion, acting as a growth and proliferative factor promoting clonal expansion of $\mathrm{T}_{\mathrm{H}} 1$ and cytotoxic $\mathrm{T}$ cells (Minami et al., 1993), and in a paracrine fashion, activating NK cells (Henney et al., 1981; Naume et al., 1993) and promoting survival of neutrophils (Djeu et al., 1993) and monocytes (Espinoza-Delgado et al., 1995). Activated $\mathrm{T}_{\mathrm{H}} 1$, cytotoxic T, and NK cells will, in turn, secrete cytokines, such as IFN- $\gamma$, which will activate and enhance intracellular killing of bacteria by macrophages.

The decrease in both IL-2 and IL-4 prior to and post-infection, and the persistent decrease in IL-2 through day 10, was not likely due to lack of T cell recruitment into the lung, as there was a significant increase in T cells responding to the infection in animal pre-treated with soluble metals on day 10 . These data, in conjunction with the apparent alteration in $\mathrm{T}$ cell function prior to infection, suggest that the soluble metals may be 
altering $\mathrm{T}$ cell activity directly, resulting in a slowing in the clearance of the bacteria over time. Whether or not this suppression in $\mathrm{T}$ cell activity is related to the ealy elevation in $\mathrm{NO}$ in the lungs of R-soluble-treated rats is unclear. Although the greatest effects of NO are on lymphocyte proliferation, it has also been shown to alter $\mathrm{T}$ cell cytokine production as well as gene transcription (Blesson et al., 2002). Interestingly, similar reductions in IL-2 and IL-4, and elvations in NO on day 6 , were not recapitulated in the animals treated with the total ROFA sample, yet there was an equivalent bacterial burden in this group when compared to the rats exposed to the soluble metals on day 10 .

Although the early inhibition of bacterial clearance in the R-Soluble and R-Total groups may be due to inhibition of macrophage activity, the later responses, which result in a similar pattern of clearance between the two groups, may be due to different mechanisms. Because the animals pre-treated with the R-Total had an similar bacterial burden as the R-Soluble animals at day 10, a stronger T cell and, consequently, AM response would have been expected at this time in order to respond to the enhanced infection. However, the T cell and AM infiltration in the R-Total group did not differ from controls. Where the soluble metals appear to be altering lymphocyte function directly at the later time points, the total ROFA may be interfering with the necessary signals between the innate and adaptive immune system earlier on, as demonstrated by a low IL-12 production, resulting in a weaker response than necessary to clear the bacterial burden present in the R-Total group. As mentioned previously, the insoluble metals alone did not alter infectivity; therefore, the differences between the soluble ROFA fraction and the total sample may be due to total metal content or to interactions between certain particulate and soluble metals in the total sample, which may alter the bioavailability or 
distribution of different metals. The presence and quantities of metals in the total sample, such as vanadium, which are absent in the soluble fraction, may act antagonistically with other metals, such as nickel, as suggested by both in vivo and in vitro studies of metal mixtures (Dreher et al., 1997; Fisher et al., 1986; Geertz et al., 1994; Kodavanti et al., 1998), which may ultimately result in different effects on AMs, NK cells, and lymphocytes.

Although the effects altering the adaptive response between the soluble metals and the total ROFA may differ, the ultimate result appears to be the same. Inhibition of AM bactericidal activity led to a high bacterial burden early in the infection, and inhibition of, delay in, or alteration of the adaptive immune response resulted in the slowing of the bacterial clearance over time. Future studies examining the effects of individual and combinations of the metals present in the soluble fraction of ROFA in the pulmonary infectivity model have been performed, and are described in study 3 , to determine the metal or metals responsible for the innate and adaptive effects observed in response to soluble ROFA fraction in this study. 


\section{Figures and Tables}
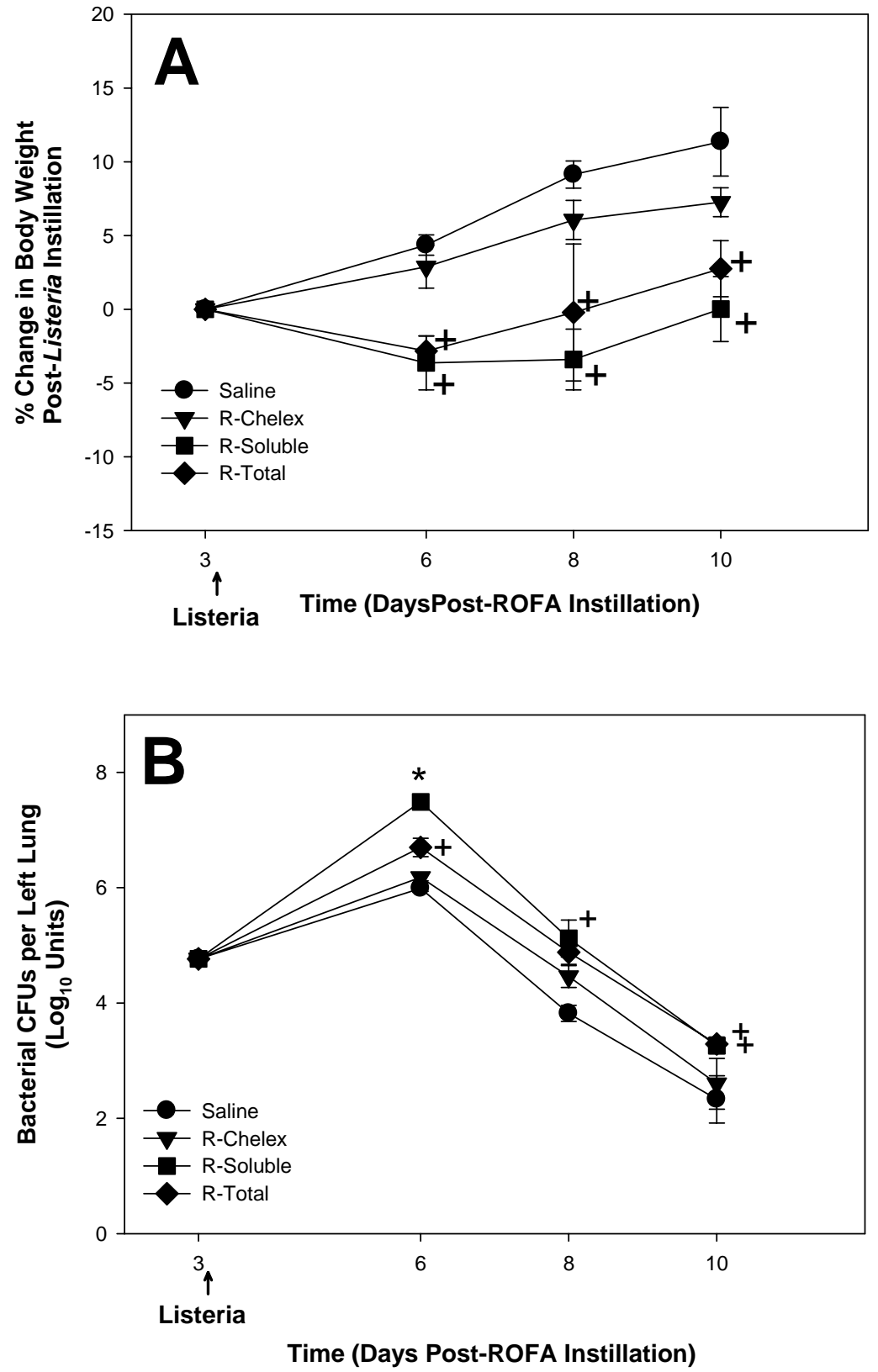

Figure 2.1. \% change in body weight post-infection (A) and number of bacterial CFUs in the left lungs (B) of rats that were pre-exposed to R-Total, R-Soluble, R-Chelex, or saline 3 days prior to intratracheal inoculation with $L$. monocytogenes. Values are means $\pm \mathrm{SE}, *$ significantly different from all groups, + significantly different from Saline and R-Chelex groups $(\mathrm{p}<0.05)$. 

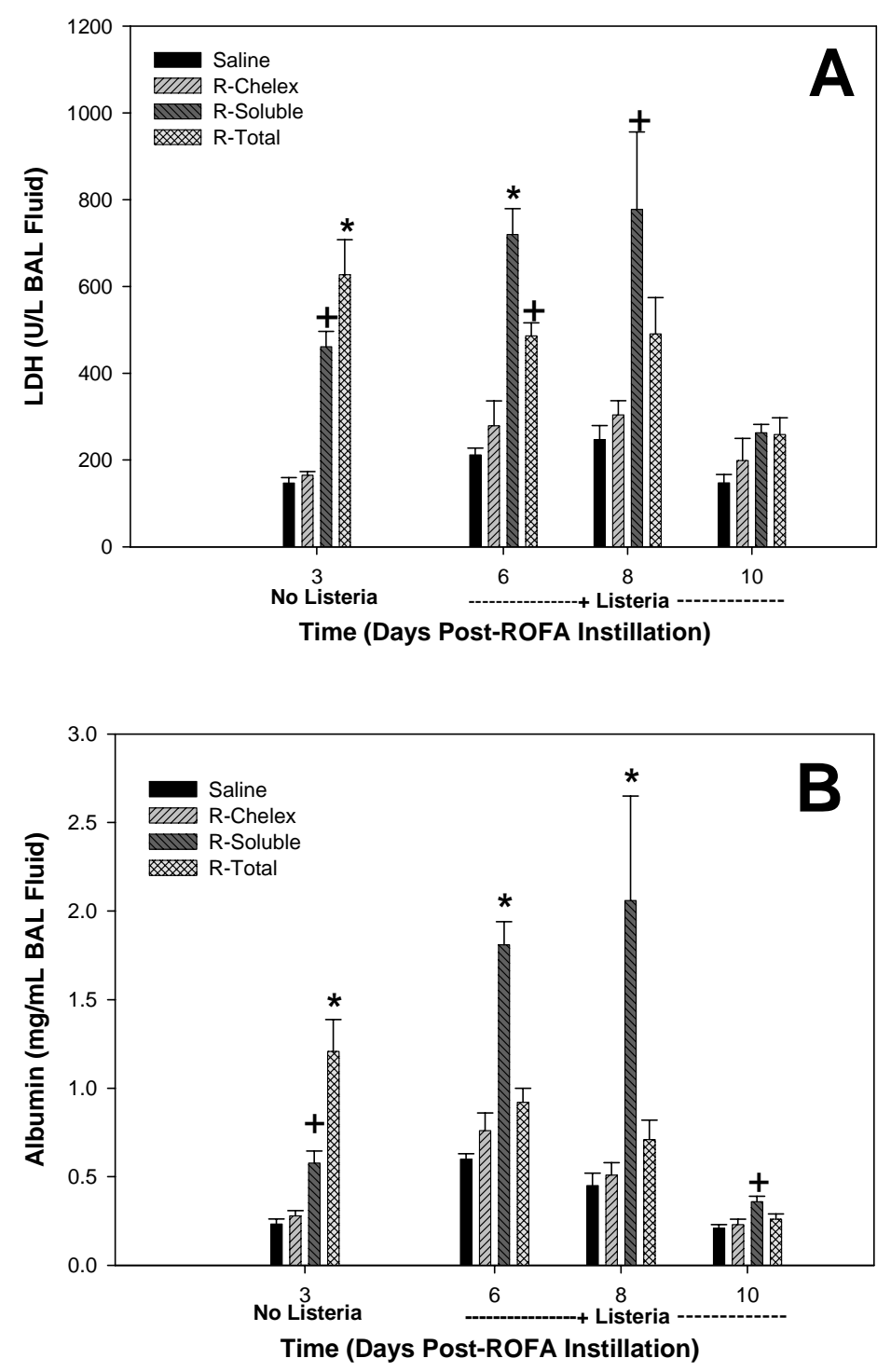

Figure 2.2. Lactate dehydrogenase (LDH) (A) and albumin (B) in the BAL fluid of rats that were pre-exposed to R-Total, R-Soluble, R-Chelex, or saline 3 days prior to intratracheal inoculation with $L$. monocytogenes. Values are means $\pm \mathrm{SE}$, * significantly different from all groups, + significantly different from Saline and R-Chelex groups $(\mathrm{p}<0.05)$. 

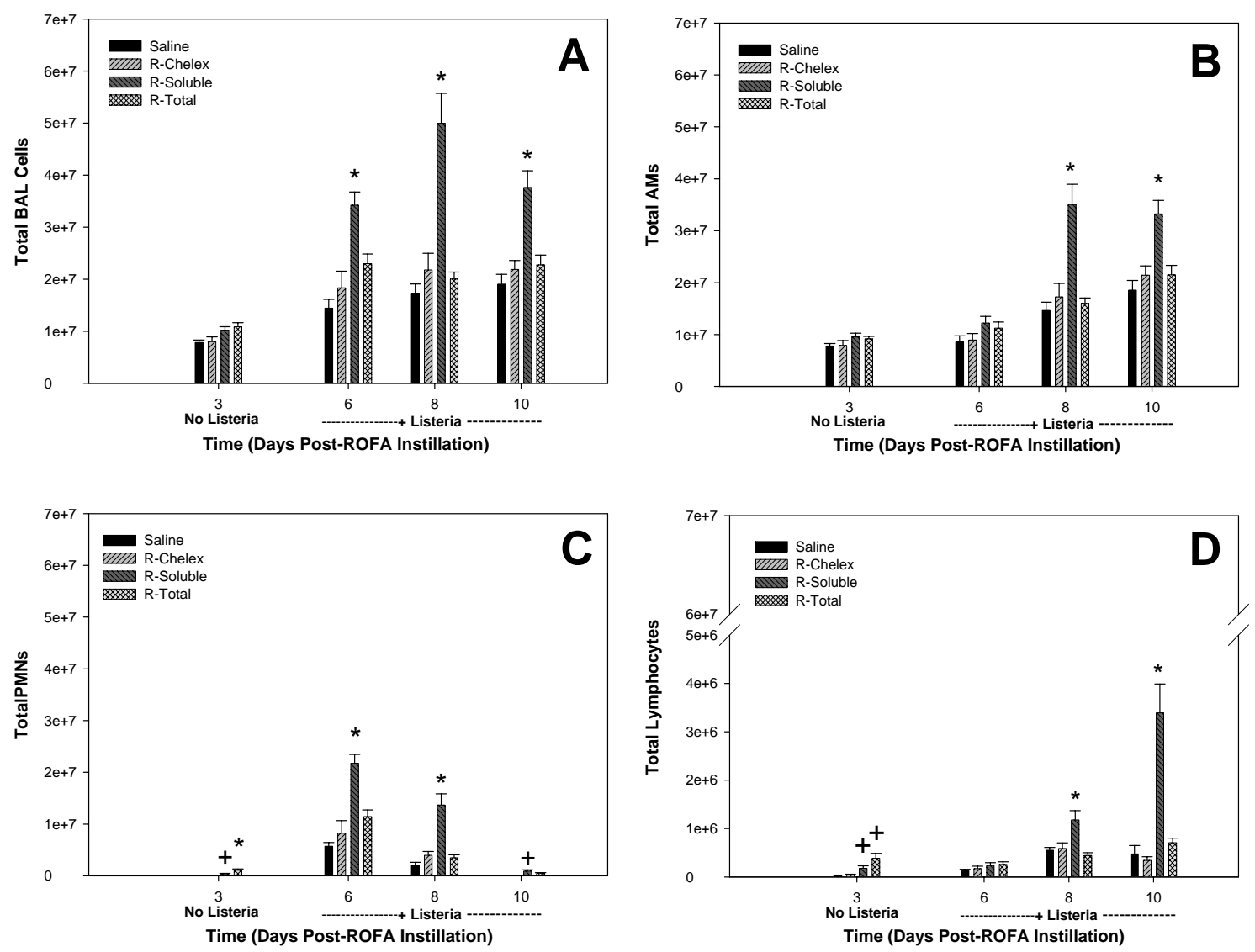

Figure 2.3. Total cells (A), totals AMs (B), total PMNs (C), and total lymphocytes (D) present in the BAL of rats that were pre-exposed to R-Total, R-Soluble, R-Chelex, or Saline 3 days prior to intratracheal inoculation with $L$. monocytogenes as determined by cell differentials. Values are means $\pm \mathrm{SE}, *$ significantly different from all groups, + significantly different from Saline and R-Chelex groups $(\mathrm{p}<0.05)$. 

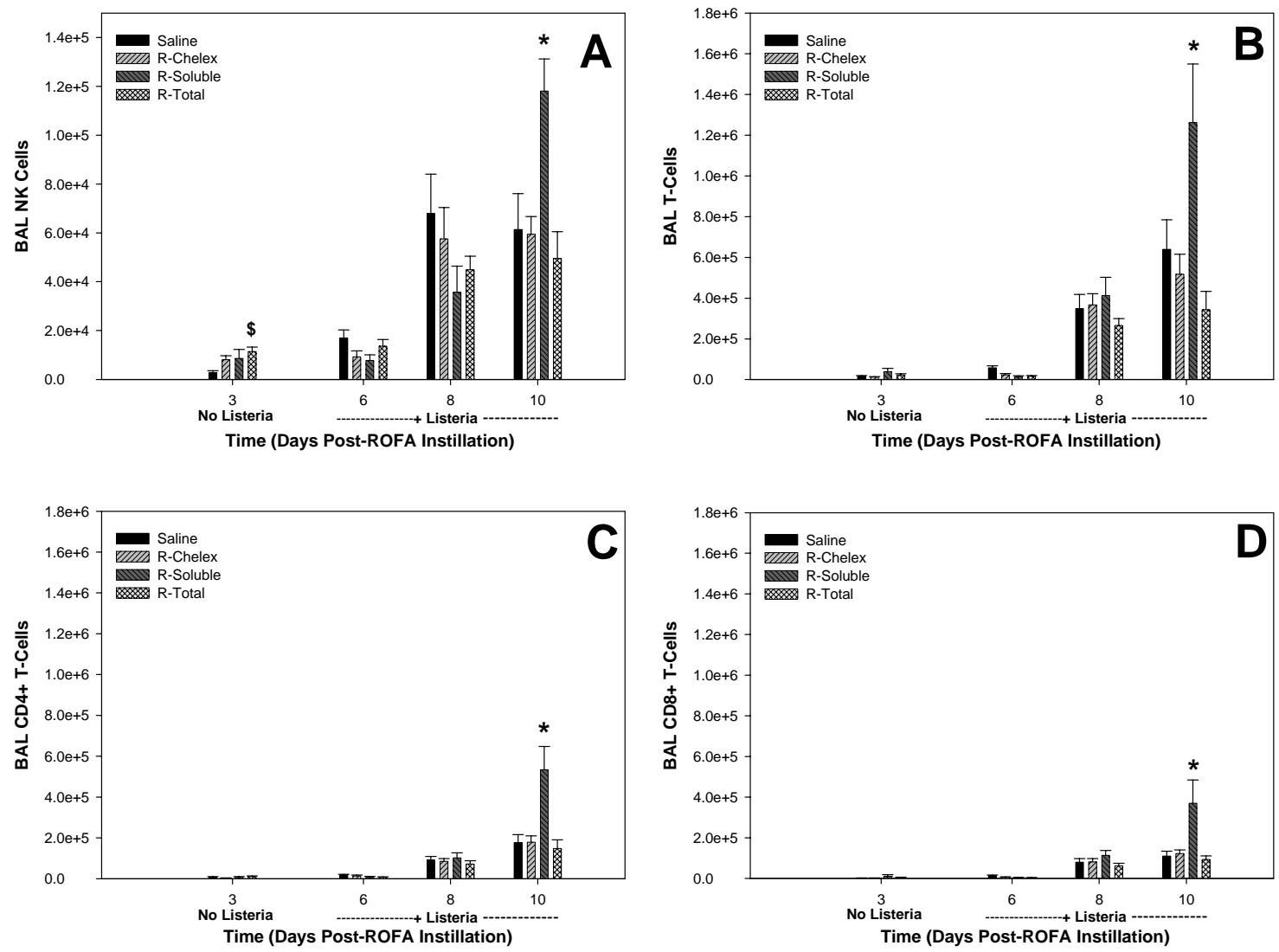

Figure 2.4: Total NK cells (A), totals T cells (B), total CD4+ T cells(C), and total CD8+ $\mathrm{T}$ cells (D) present in the BAL of rats that were pre-exposed to R-Total, R-Soluble, RChelex, or Saline 3 days prior to intratracheal inoculation with $L$. monocytogenes as determined by flow cytometry. Values are means \pm SE, * significantly different from all groups, + significantly different from Saline and R-Chelex groups, \$ significantly different from the Saline group $(\mathrm{p}<0.05)$. 

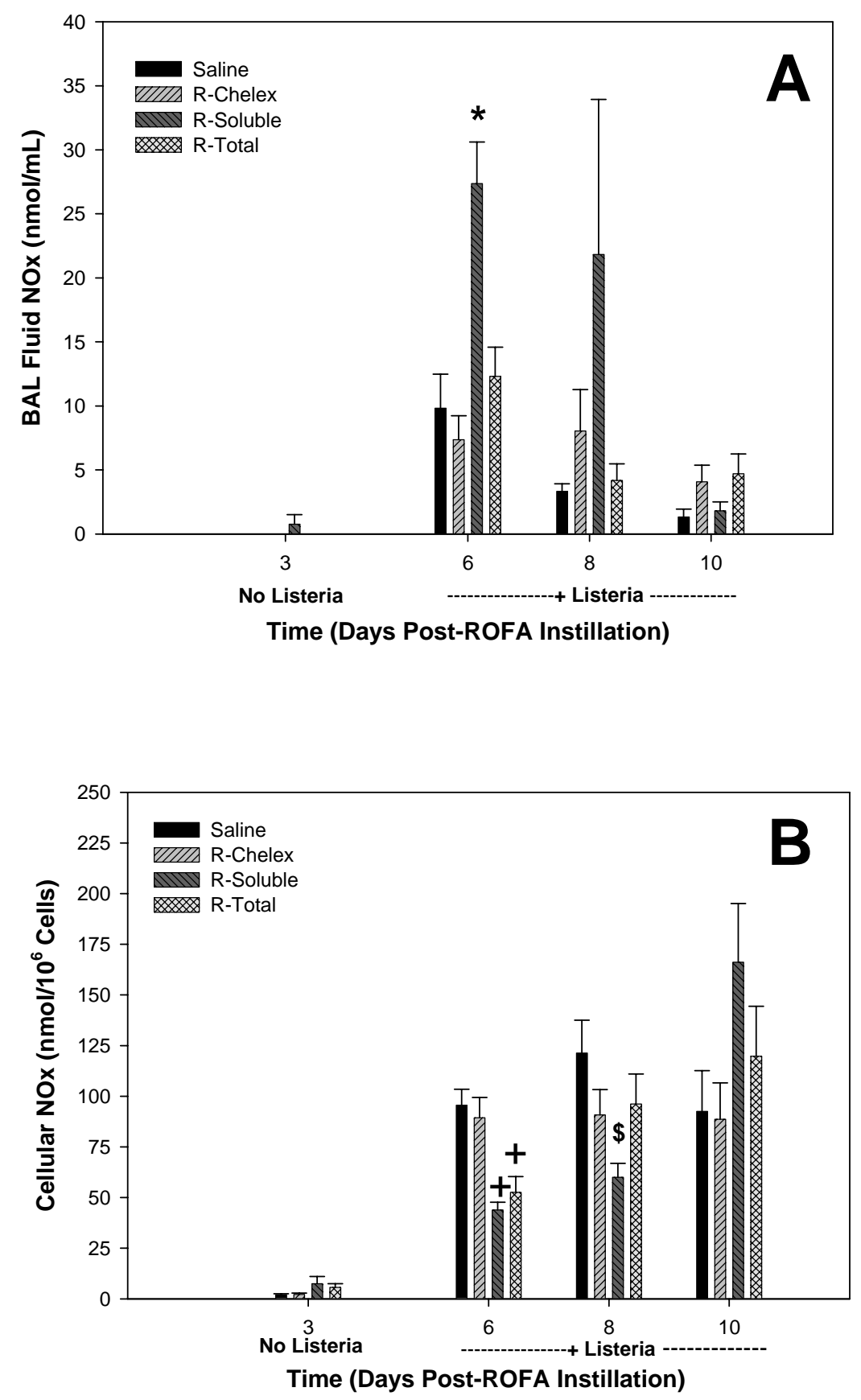

Figure 2.5. Nitrate and nitrite (NOx) in the BAL fluid (A) and cell media from BAL cells cultured for 18 hours (B) from rats that were pre-exposed to R-Total, R-Soluble, RChelex, or Saline 3 days prior to intratracheal inoculation with L. monocytogenes. Values are means $\pm \mathrm{SE}$, * significantly different from all groups; + significantly different from Saline and R-Chelex groups; \$ significantly different from the Saline group $(\mathrm{p}<0.05)$. 

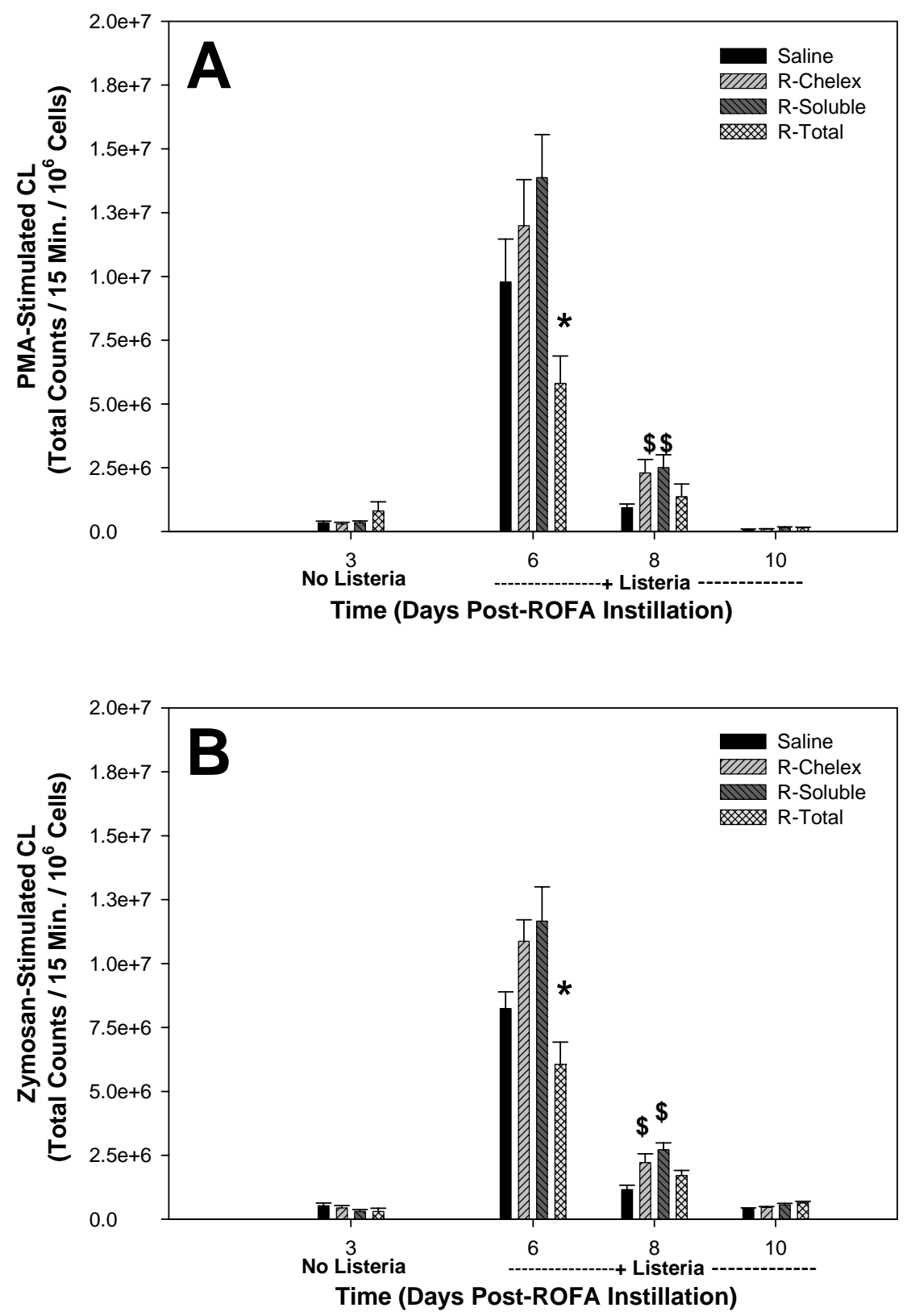

Figure 2.6. BAL cellular chemiluminescence (CL) depicted in total counts per $15 \mathrm{~min}$ per $1 \times 10^{6}$ cells after stimulation with PMA (A) or zymosan (B) in rats that were preexposed to R-Total, R-Soluble, R-Chelex, or Saline 3 days prior to intratracheal inoculation with $L$. monocytogenes. Values are means $\pm \mathrm{SE}, *$ significantly different from all groups, $\$$ significantly different from the Saline group $(\mathrm{p}<0.05)$. 

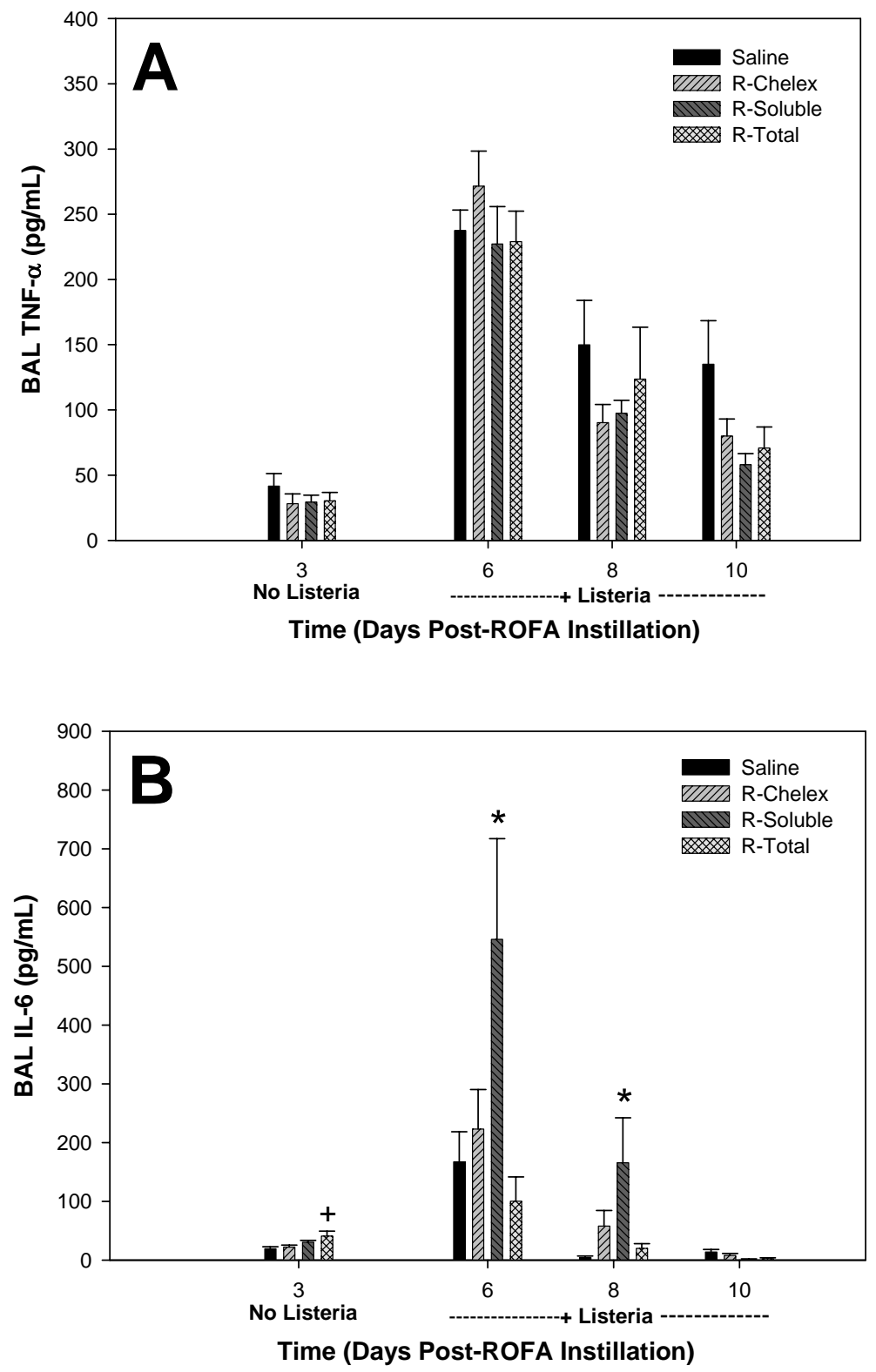

Figure 2.7. Concentration of pro-inflammatory cytokine, TNF- $\alpha$ (A), and acute phase response cytokine, IL-6 (B), in the BAL fluid of rats that were pre-exposed to R-Total, RSoluble, R-Chelex, or Saline 3 days prior to intratracheal inoculation with $L$. monocytogenes. Values are means $\pm \mathrm{SE}, *$ significantly different from all groups; + significantly different from Saline and R-Chelex groups $(\mathrm{p}<0.05)$. 


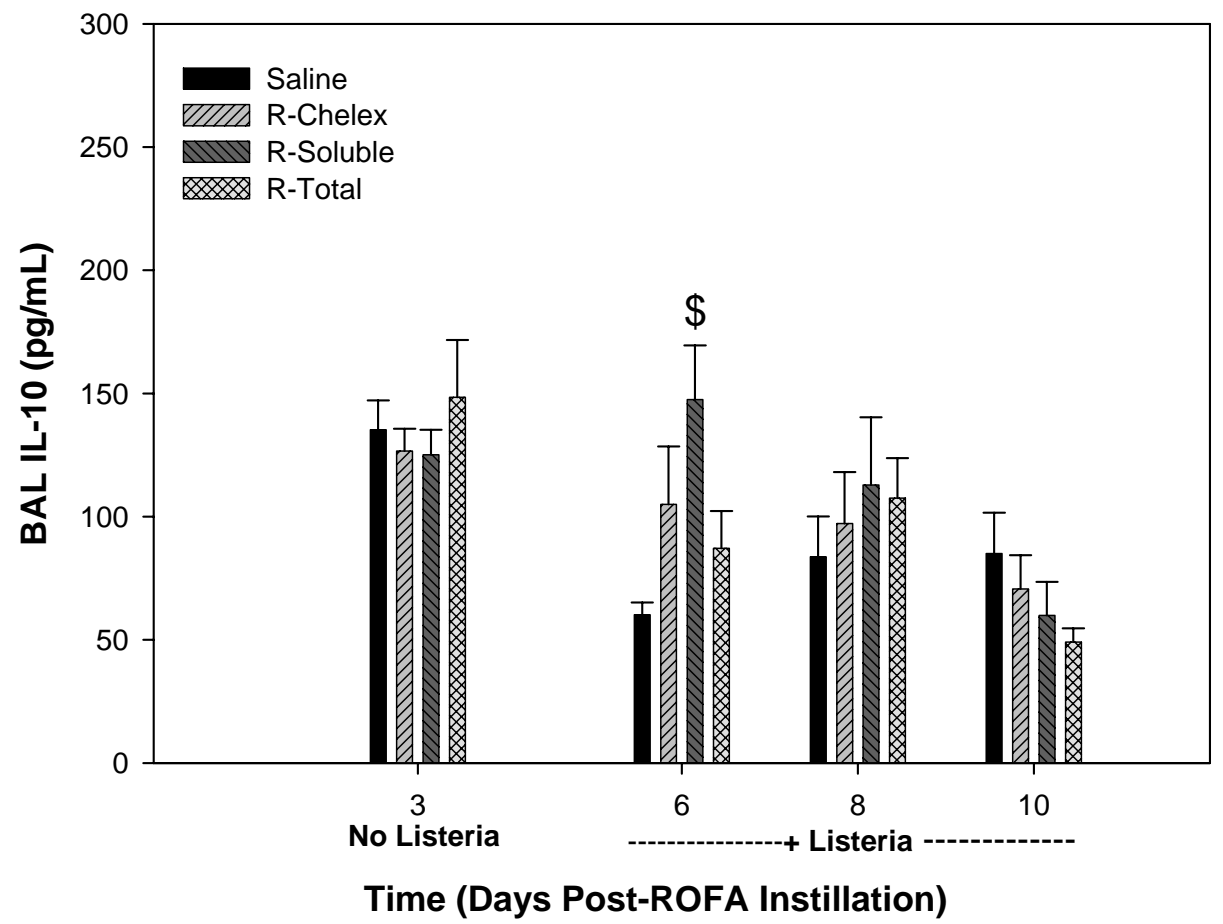

Figure 2.8. IL-10, an anti-inflammatory cytokine, in the BAL fluid of rats that were preexposed to R-Total, R-Soluble, R-Chelex, or Saline 3 days prior to intratracheal inoculation with $L$. monocytogenes. Values are means \pm SE, $\$$ significantly different from the Saline group $(\mathrm{p}<0.05)$. 

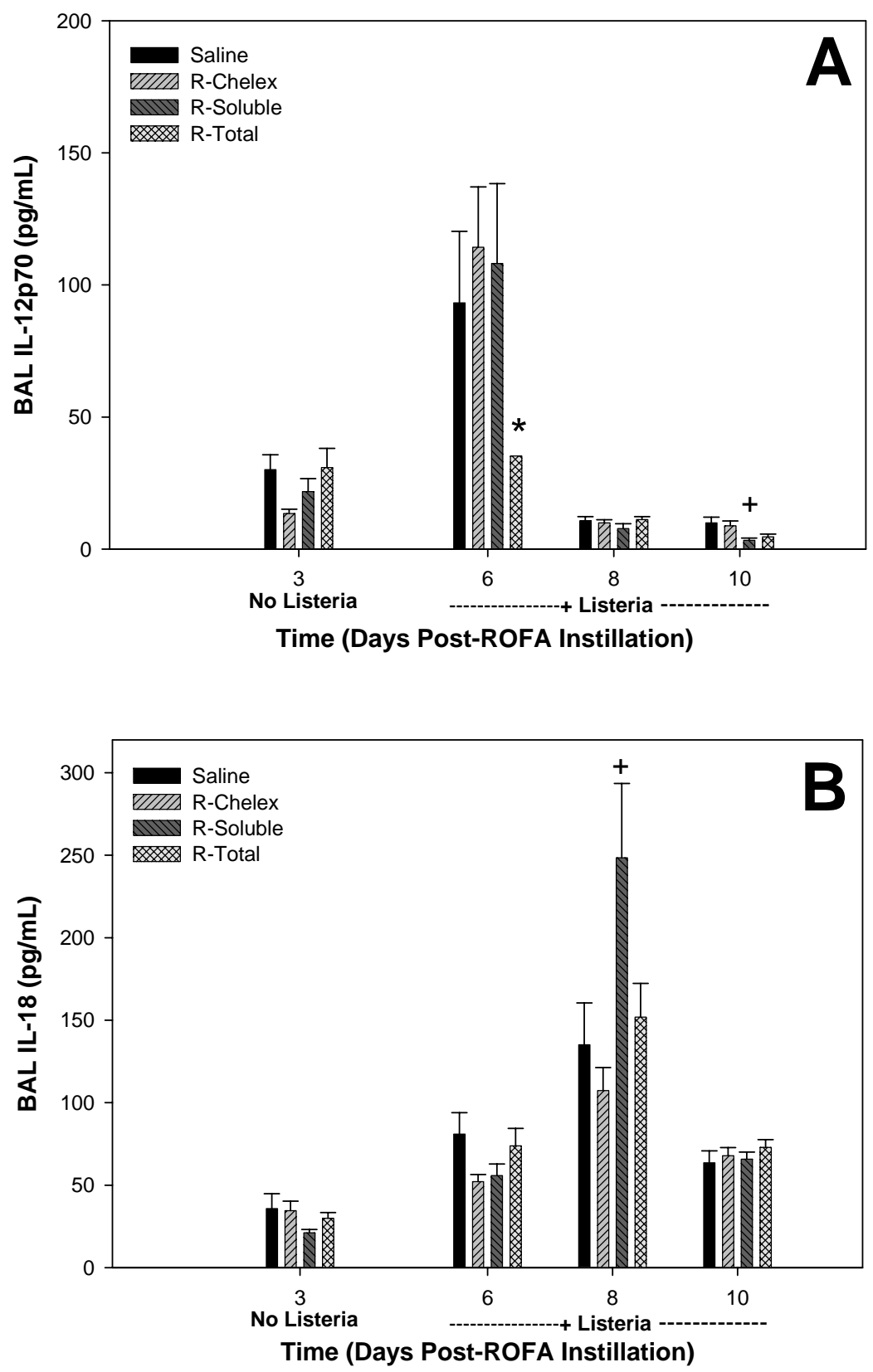

Figure 2.9. Cytokines involved in directing the appropriate T cell response to $L$. monocytogenes, IL-12p70 (A) and IL-18 (B), in the BAL fluid of rats that were preexposed to R-Total, R-Soluble, R-Chelex, or Saline 3 days prior to intratracheal inoculation with $L$. monocytogenes. Values are means $\pm \mathrm{SE}, *$ significantly different from all groups, + significantly different from R-Chelex and Saline group $(\mathrm{p}<0.05)$. 

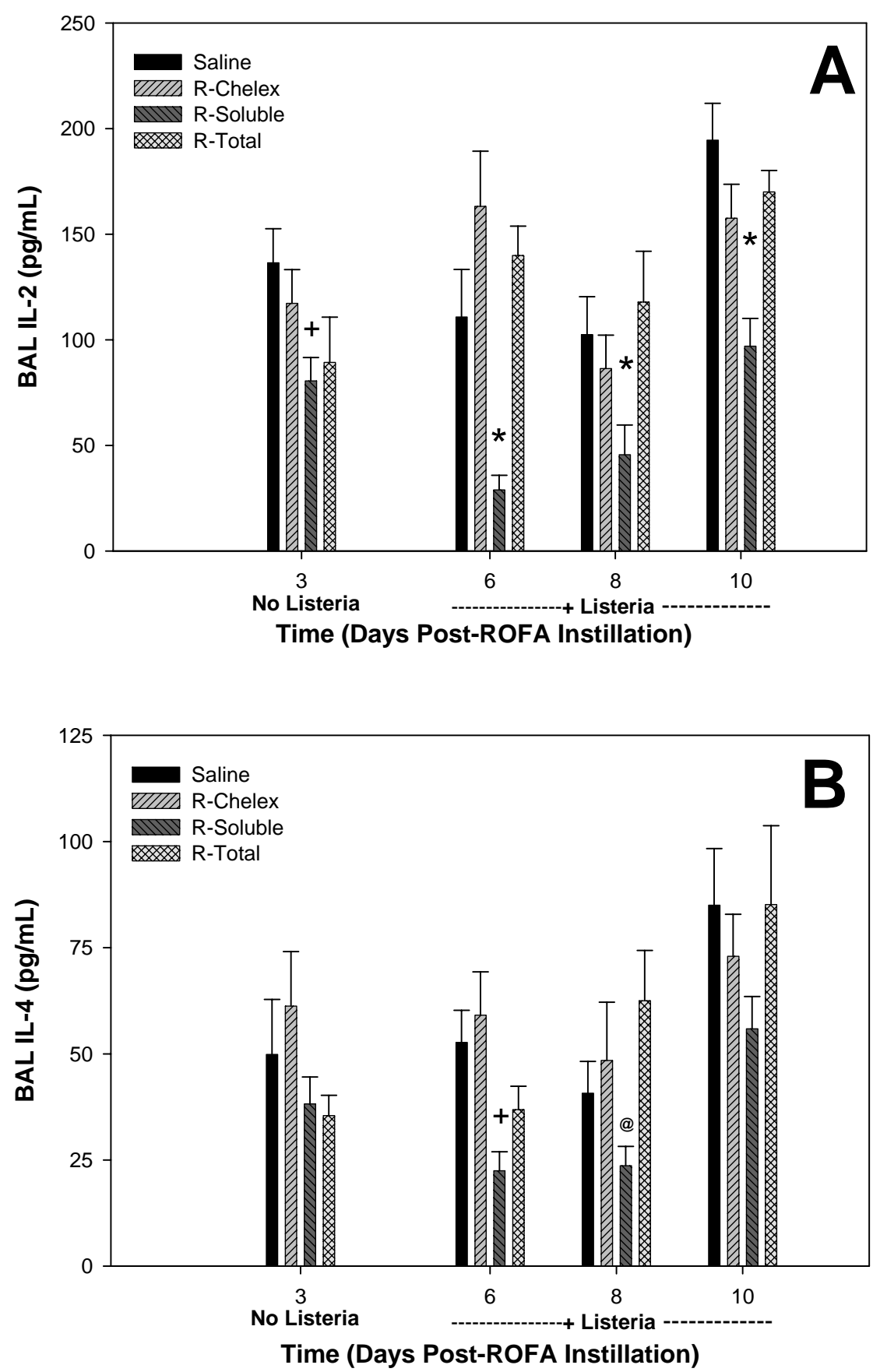

Figure 2.10. T cell cytokines, IL-2 (A) and IL-4 (B), in the BAL fluid of rats that were pre-exposed to R-Total, R-Soluble, R-Chelex, or Saline 3 days prior to intratracheal inoculation with $L$. monocytogenes. Values are means $\pm \mathrm{SE}$, * significantly different from all groups, + significantly different from R-Chelex and Saline groups, ${ }^{@}$ significantly different from the $\mathrm{R}$-Total group $(\mathrm{p}<0.05)$. 
Table 2.1. T/B Cell Ratio

$\underline{\text { Day } 3} \quad \underline{\text { Day } 6} \quad \underline{\text { Day } 8} \quad \underline{\text { Day10 }}$

$\begin{array}{lcccc}\text { Saline } & 2.4 \pm 1.2 & 0.69 \pm 0.09 & 1.9 \pm 0.33 & 8.9 \pm 2.9 \\ \text { R-Chelex } & 2.0 \pm 0.80 & 0.65 \pm 0.21 & 3.4 \pm 0.85 & 7.6 \pm 1.8 \\ \text { R-Soluble } & 2.3 \pm 0.31 & 1.0 \pm 0.48 & 4.2 \pm 0.39^{\$} & 8.8 \pm 1.5 \\ \text { R-Total } & 1.6 \pm 0.26 & 0.71 \pm 0.09 & 2.3 \pm 0.20 & 7.0 \pm 1.6\end{array}$

Table 2.1. Ratio of T cells to B cells retrieved from the BAL of rats that were preexposed to R-Total, R-Soluble, R-Chelex, or Saline 3 days prior to intratracheal inoculation with L. monocytogenes. Values are means \pm SE, $\$$ significantly different from the Saline group $(\mathrm{p}<0.05)$. 


\section{Table 2.2. CD4+/CD8+ T Cell Ratio}

$\underline{\text { Day } 3} \quad \underline{\text { Day } 6} \quad \underline{\text { Day } 8} \quad \underline{\text { Day10 }}$

$\begin{array}{lcccc}\text { Saline } & 4.4 \pm 0.81 & 1.6 \pm 0.34 & 1.2 \pm 0.11 & 1.9 \pm 0.36 \\ \text { R-Chelex } & 2.5 \pm 0.47 & 1.9 \pm 0.60 & 1.3 \pm 0.25 & 1.6 \pm 0.19 \\ \text { R-Soluble } & 2.9 \pm 0.64 & 1.2 \pm 0.29 & 1.1 \pm 0.19 & 1.4 \pm 0.19 \\ \text { R-Total } & 2.5 \pm 0.62 & 2.3 \pm 0.78 & 1.3 \pm 0.18 & 1.5 \pm 0.23\end{array}$

Table 2.2. Ratio of CD4+ $\mathrm{T}$ cells to $\mathrm{CD} 8+\mathrm{T}$ cells retrieved from the BAL of rats that were pre-exposed to R-Total, R-Soluble, R-Chelex, or Saline 3 days prior to intratracheal inoculation with $L$. monocytogenes. Values are means $\pm \mathrm{SE}(\mathrm{p}<0.05)$. 


\section{References}

Akira, S., Hirano, T., Taga, T., and Kishimoto, T. 1990. Biology of multifunctional cytokines: IL-6 related molecules (IL-1 and TNF). FASEB J. 4:2860-2867.

American Thoracic Society, Committee of the Environmental and Occupational Health Assembly, Bascom, R., Bromberg, P. A., Costa, D. A., Devlin, R., Dockery, D. W., Frampton, M. W., Lambert, W., Samet, J. M., Speizer, F. E., and Utell, M. 1996. Health effects of outdoor air pollution. Part 1. Am. J. Respir. Crit. Care Med. 153:3-50.

Antonini, J. M., Van Dyke, K., Ye, Z., DiMatteo, M., and Reasor, M. J. 1994. Introduction of luminal-dependent chemiluminescence as a method to study silica inflammation in the tissue and phagocytic cells of rat lung. Environ. Health Perspect. 102 (Suppl. 10): 37-42.

Antonini, J. M., Roberts, J. R., Jernigan, M. R., Yang, H-M., Ma, J. Y. C., and Clarke, R. W. 2002. Residual oil fly ash increases the susceptibility to infection and severely damages the lungs after pulmonary challenge with a bacterial pathogen. Toxicol. Sci. 70:110-119.

Antonini, J. M., Taylor, M. D., Millecchia, L., Bebout, A., R., and Roberts, J. R. 2004. Suppression in lung defense responses after bacterial infection in rats pretreated with different welding fumes. Toxicol. Appl. Pharmacol. 200: 206-218.

Bancroft, G. J., Schreiber, R. D., and Unanue, E. R. 1991. Natural immunity: a T-cellindependent pathway of macrophage activation, defined in the SCID mouse. Immun. Rev. 124: 5-24.

Becker, S., Soukup, J. M., and Gallagher, J. E. 2002. Differential particulate air pollution induced oxidant stress in human granulocytes, monocytes, and alveolar macrophages. Toxicol. In Vitro. 16:209-218.

Berg, R. E., Cordes, C. J., and Forman, J. 2002. Contribution of CD8+ T cells to innate immunity: IFN- $\gamma$ secretion induced by IL-12 and IL-18. Eur. J. Immunol. 32: 2807-2816.

Billiau, A. 1996. Interferon-gamma: biology and role in pathogenesis. Adv. Immunol. 62: 61-130.

Blesson, S., Thiery, J., Gaudin, C., Stancou, R., Kolb, J-P., Moreau, J-L., Theze, J., Mami-Chouaib, F., and Chouaib S. 2002. Analysis of the mechanism of human cytotoxic T lymphocyte response inhibition by NO. Internat. Immunol. 14: 1169-1178.

Borish, L., Rosenbaum, R., Albury, L., and Clark, S. 1989. Activation of neutrophils by recombinant interleukin 6. Cell. Immunol. 121: 280-289. 
Brain, J. D., Knudson, D. E., Sorokin, S. P., and Davis, M. A. 1976. Pulmonary distribution of particles given by intratracheal instillation or by aerosol inhalation. Environ. Res. 11:13-33.

Castranova, V., Jones, T., Barger, M., Afshari, A., and Frazer, D. J. 1990. Pulmonary responses of guinea pigs to consecutive exposures to cotton dust. Proc. $14^{\text {th }}$ Cotton Dust Research Conference, eds. Jacobs, R. R., Wakelyn, P. J., Domelsmith, and L. N. pp 131135. Memphis, TN: National Cotton Council.

Chan, S. H., Kobayashi, M., Santoli, D., Perussia, B., and Trinchieri, G. 1992. Mechanisms of IFN-gamma induction by natural killer cell stimulatory factor (NKSF/IL12). Role of transcription and mRNA stability in the synergistic interaction between NKSF and IL-2. J. Immunol. 148: 92-98.

Clarke, R. W., Catalano, P. J., Koutrakis, P., Murthy, G. G. K., Sioutas, C., Paulauskis, J., Coull, B., Ferguson, S., and Godleski, J. J. 1999. Urban air particulate inhalation alters pulmonary function and induces pulmonary inflammation in a rodent model of bronchitis. Inhal. Toxicol. 11: 637-656.

Cohen, M. D., Sisco, M., Zelikoff, J. T., and Schlesinger, R. B. 2001. Ozone-induced modulation of cell-mediated immune responses in the lungs. Toxicol. Appl. Pharmacol. 171: 71-84.

Dalrymple, S. A., Lucian, L. A., Slattery, R., McNeil, T., Aud, D. M., Fuchino, S., Lee, F., and Murray, R. 1995. Interleukin-6-deficient mice are highly susceptible to Listeria monocytogenes infection: correlation with inefficient neutrophilia. Infect. Immun.. 63: 2262-2268.

Djeu, J. Y., Liu, J. H., Wei, S., Rui, H., Pearson, C. A., Leonard, W. J., and Blanchard, D. K. 1993. Function associated with IL-2 receptor-beta on human neutrophils. Mechanism of activation of antifungal activity against Candida albicans by IL-2. J. Immunol. 150: 960-970.

Dormans, J. A., Steerenberg, P. A., Arts, J. H., van Bree, L., de Klerk, A., Verlaan, A. P., Bruijntjes, J. P., Beekhof, P., van Soolingen, D., and van Loveren, H. 1999. Pathological and immunological effects of respirable coal fly ash in male Wistar rats. Inhal Toxicol. 11: 51-69.

Dreher, K. L., Jaskot, R. H., Lehmann, J. R., Richards, J. H., McGee, J. K., Ghio, A. J., and Costa, D. L. 1997. Soluble transition metals mediate residual oil fly ash-induced acute lung injury. J. Toxicol. Environ. Health. 50:285-305.

Dye, J. A., Adler, K. B., Richards, J. H., and Dreher, K. L. 1999. Role of soluble metals in oil fly ash-induced airway epithelial injury and cytokine gene expression. Am. J. Physiol. 277:L498-L510. 
Dye, J. A., Lehmann, J. R., McGee, J. K., Winsett, D. W., Ledbetter, A. D., Everitt, J. I., Ghio, A. J, and Costa, D. L. 2001. Acute pulmonary toxicity of particulate matter filter extracts in rats: coherence with epidemiologic studies in Utah Valley residents. Environ. Health Perspect. 109(Suppl 3): 395-403.

Emmerling, P., Finger, H., and Boekeml, J. 1975. Listeria monocytogenes infection in nude mice. Infect. Immun. 12: 437-439.

Environmental Protection Agency. 2004. The Particle Pollution Report: Current understanding of air quality and emissions through 2003. pp 1-27.

Espinoza-Delgado, I., Bosco, M. C., Musso, T., Gusella, G. L., Longo, D. L., and Varesio, L. 1995. Interleukin-2 and human monocyte activation. J. Leukoc. Biol. 57: 139.

Fisher, G. L., McNeill, K. L., and Democko, C. J. 1986. Trace element interactions affecting pulmonary macrophage cytotoxicity. Environ. Res. 39: 164-171.

Ford, H. R., Hoffman, R. A., Wang, S., and Simmons, R. L. 1991. Induction of cytotoxic T lymphocyte development from murine thymocytes by IL-1 and IL-6. J. Pediatr. Surg. 26: 397-400.

Gao, F., Barchowsky, A., Nemec, A. A., and Fabisiak, J. P. 2004. Microbial stimulation by Mycoplasma fermentans synergistically amplifies IL-6 release by human lung fibroblasts in response to residual oil fly ash (ROFA) and nickel. Toxicol. Sci. 81: 467479.

Gavett, S. H., Madison, S. L., Dreher, K. L., Winsett, D. W., McGee, J. K., and Costa, D. L. 1997. Metal and sulfate composition of residual oil fly ash determines airway hyperreactivity and lung injury in rats. Environ. Res. 72:162-172.

Gavett, S. H., Madison, S. L., Stevens, M. A., and Costa, D. L. 1999. Residual oil fly ash amplifies allergic cytokines, airway responsiveness, and inflammation in mice. Am. J. Respir. Crit. Care Med. 160: 1897-1904.

Geertz, R., Gulyas, H., and Gercken, G. 1994. Cytotoxicity of dust constituents toward alveolar macrophages: Interaction of heavy metal compounds. Toxicology. 86: 13-27.

Ghio, A. J. 2004. Biological effects of Utah Valley ambient air particles in humans: a review. J. Aerosol Med. 17: 157-164.

Goldsmith, C. A., Imrich, A., Danaee, H., Ning, Y., and Kobzik, L. 1998. Analysis of air pollution particulate-mediated oxidant stress in alveolar macrophages. J. Toxicol. Environ. Health. Part A. 54: 529-545. 
Goldsmith, C. A., Hamada, K., Ning, Y. Y., Qin, P., Murthy, G. G. K., Lawrence, J. and Kobzik, L. 1999. Effects of environmental aerosols on airway hyperresponsiveness in a murine model of asthma. Inhal. Toxicol. 11:981-998.

Green, L.C., Wagner, C. A., Glogowski, J., Skipper, P.L., Wishnok, J. S., and Tannenbaum, S. R. 1982. Analysis of nitrate, nitrite, and $\left[{ }^{15} \mathrm{~N}\right]$ nitrate in biological fluids. Anal. Biochem. 126: 131-138.

Green, S. J., Scheller, L. F., Marletta, M. A., Seguin, M. C., Klotz, F. W., Slayter, M., Nelson, B. J., and Nacy, C. A. 1994. Nitric oxide: cytokine-regulation of nitric oxide in host resistance to intracellular pathogens. Immunol. Letters. 43: 87-94.

Hatch, G. E., Boykin, E., Graham, J. A., Lewtas, J., Pott, F., Loud, K., and Mumfor, J. L. 1985. Inhalable particles and pulmonary host defense: In vivo and in vitro effects of ambient air and combustion particles. Environ. Res. 36:67-80.

Henney, C. S., Kuribayashi, K., Kern, D. E., and Gillis, S. 1981. Interleukin-2 augments natural killer cell activity. Nature. 291: 335-8.

Hirano, T., Akira, S., Taga, T., and Kishimoto, T. 1990. Biological and clinical aspects of interleukin 6. Immunol. Today. 11: 443-449.

Hoffman, R. A., Mahidhara, R. S., Wolf-Johnston, A. S., Lu, L., Thomsaon, A. W., and Simmons, R. L. 2002. Differential modulation of CD4 and CD8 T cell proliferation by induction of nitric oxide synthase in antigen presenting cells. Transplantation. 74:836845.

Hsieh, C. S., Macatonia, S. E., Tripp, C. S., Wolf, S. F., O'Garra, A., and Murphy, K. M. 1993. Development of $\mathrm{T}_{\mathrm{H}} 1 \mathrm{CD} 4+\mathrm{T}$ cells through IL-12 produced by Listeria-induced macrophages. Science. 260: 547-9.

Jakab, G. J. 1993. The toxicologic interactions resulting from inhalation of carbon black and acrolein on pulmonary antibacterial and antiviral defenses. Toxicol. Appl. Pharmacol. 121: 167-175.

Janeway, C. A., Travers, P., Walport, M., and Shlomchik, M. Eds. 2001. In: Immunobiology. $5^{\text {th }}$ Edition. Garland Publishing, New York, NY. Chapter 8.

Kaufmann, S. H., Emoto, M., Szalay, G., Barsig, J., and Flesch, I. E. 1997. Interleukin-4 and listeriosis. Immunol. Rev. 158:95-105.

Kodavanti, U. P., Hauser, R., Christiani, D. C., Meng, Z. H., McGee, J., Ledbetter, A., Richards, J., and Costa, D. L. 1998. Pulmonary responses to oil fly ash particles in the rat differ by virtue of their specific soluble metals. Toxicol. Sci. 43:204-212. 
Kopf, M., Baumann, H., Freer, G., Freudenberg, M., Lamers, M., Kishimoto, T., Zinkernagel, R., Bluethmann, H., and Kohler, G. 1994. Impaired immune and acutephase responses in interleukin-6-deficient mice. Nature. 368: 339-342.

Kradin, R. L., Liu, H-W., van Rooijen, N., Springer, K., Zhao, L-H., and Leary, C. P. 1999. Pulmonary immunity to Listeria is enhanced by elimination of alveolar macrophages. Am. J. Respir. Crit. Care Med. 159: 1967-1974.

Lambert, A. L., Dong, W., Selgrade, M. K., and Gilmour, M. I. 2000. Enhanced allergic sensitization by residual oil fly ash particles is mediated by soluble metal constituents. Toxicol. Appl. Pharmacol.165: 84-93.

Leonardi, G. S., Houthuijs, D., Steerenberg, P. A., Fletcher, T., Armstrong, B., and Antova, T. 2000. Immune biomarkers in relation to exposure to particulate matter: a cross-sectional survey in 17 cities in central Europe. Inhal. Toxicol.12(Suppl 4): 1-14.

Lewis, A. B., Taylor, M. D., Roberts, J. R., Leonard, S. S., Shi, X., and Antonini, J. M. 2003. Role of metal-induced reactive oxygen species generation in lung responses caused by residual oil fly ash. J. Biosci. 28:13-18.

Lotz, M., Jirik, F., Kabouridis, R. Tsoukas, C., Hirano, T., Kishimoto, T., and Carson, D. A. 1988. B cell stimulating factor/interleukin 6 is a costimulant for human thymocytes and T lymphocytes. J. Exp. Med. 167: 1253-1258.

MacLean, J. A., Xia, W., Pinto, C. E., Zhao, L., Liu, H. W., and Kradin, R. L. 1996. Sequestration of inhaled particulate antigens by lung phagocytes. A mechanism for the effective inhibition of pulmonary cell-mediated immunity. Am. J. Pathol. 148: 657-666.

MacMicking, J. D., Nathan, C., Hom, G., Chartrain, N., Fletcher, D. S., Trumbauer, M., Stevens, K., Xie, Q. W., Sokol, K., Hutchinson, N., Chen, H. and Mudget, J. S. 1995. Altered responses to bacterial infection and endotoxic shock in mice lacking inducible nitric oxide synthase. Cell. 81: 641-650.

Minami, Y., Kono, T., Miyazaki, T., and Taniguchi, T. 1993. The IL-2 receptor complex: its structure, function, and target genes. Ann. Rev. Immunol. 11: 245-68.

Moore, K. W., de Waal Malefyt, R., Coffman, R. L., and O'Garra, A. 2001. Interleukin10 and the interleukin-10 receptor. Annu. Rev. Immunol.19:683-765.

Mosmann, T. R., and Sad, S. 1996. The expanding universe of T-cell subsets: Th1, Th2, and more. Immunol. Today. 17:138-146.

Nacy, C. A., Meierovics, A. I., Belosevic, M., and Green, S. J. 1991. Tumor necrosis factor-alpha: central regulatory cytokine in the induction of macrophage antimicrobial activities. Pathobiol. 59: 182-184. 
Naume, B., Johnsen, A. C., Espevik, T., and Sundan, A. 1993. Gene expression of cytokines and cytokine receptors from highly purified CD56+ natural killer cells stimulated with interleukin-2, interleukin-7, and interleukin-12. Eur. J. Immunol. 23: 1831-1838.

Neighbors, M., Xu, X., Barrat, F. J., Ruuls, S. R., Churakova, T. Debets, R., Bazan, J. F., Kastelein, R. A., Abrams, J. S., and O'Garra, A. 2001. A critical role for interleukin 18 in primary and memory effector responses to Listeria monocytogenes that extends beyond its effects on interferon $\gamma$ production. J. Exp. Med. 194: 343-354.

Nickol, A. D., and Bonventre, P. F. 1977. Anomalous high native resistance of athymic mice to bacterial pathogens. Infect. Immun. 18: 636-645.

NIOSH. 1994. Elements (ICP): Method 7300. In NIOSH Manual of Analytical Methods, $4^{\text {th }}$ Ed., Issue 2, pp. 1-10. U.S. Department of Health and Human Services, Publication No. 98-119. NIOSH, Washington, DC.

North, R. J., Dunn, P. L., and Conlan, J. W. 1997. Murine listeriosis model of antimicrobial defense. Immunol. Rev. 158: 27-36.

Okamura, H., Tsutsi, H., Komatsu, T., Yutsudo, M., Hakura, A., Tanimoto, T., Torigoe, K., Okura, T., Nukada, Y., Hattori, K., Akita, K., Namba, M., Tanabe, F., Konishi, K., Fukuda, S., and Kurimoto, M. 1995. Cloning of a new cytokine that induces IFN-gamma production by T cells. Nature. 378:88-91.

Omara, F. O., Fournier, M., Vincent, R., and Blakley, B. R. 2000. Suppression of rat and mouse lymphocyte function in urban air particulates (Ottawa dust) is reversed by Nacetylcysteine. J. Toxicol. Environ. Health Part A. 59: 67-85.

Pamer, E. G. 2004. Immune responses to Listeria monocytogenes. Nature Rev. 4:812823.

Paul, W. E., and Seder, R. A. 1994. Lymphocyte responses and cytokines. Cell. 76:241251.

Pritchard, R., Ghio, A. J., Lehmann, J. R., Winsett, D.W., Tepper, J. S., Park, P., Gilmour, M. I., Dreher, K. L., and Costa, D. L. 1996. Oxidant generation and lung injury after particulate air pollution exposure increase with the concentration of associated metals. Inhal. Toxicol. Inhal. Toxicol. 8:457-477.

Redpath, S., Ghazal, P., and Gascoigne, N. R. 2001. Hijacking and exploitation of IL-10 by intracellular pathogens. Trends Microbiol. 9: 86-92.

Renauld, J. C., Vink, A., and Van Snick, J. 1989. Accessory signals in murine cytolytic T cell responses: dual requirement for IL-1 and IL-6. J. Immunol.143: 1894-1898. 
Roberts, E. S., Richards, J. H., Jaskot, R., and Dreher, K. L. 2003. Oxidative stress mediates air pollution particle-induced acute lung injury and molecular pathology. Inhal. Toxicol. 15: 1327-1346.

Schaumann, F., Borm, P. J. A., Herbrich, A., Knoch, J., Pitz, M., Schins, R. P. F., Luettig, B., Hohlfeld, J. M., Heinrich, J., and Krug, N. 2004. Metal-rich ambient particles (particulate matter 2.5 ) cause airway inflammation in healthy subjects. Am. J. Respir. Crit. Care Med. 170: 898-903.

Seder, R. A., and Gazzinelli, R. T. 1999. Cytokines are critical in linking the innate and adaptive immune responses to bacterial, fungal, and parasitic infection. Adv. Intern. Med. 44: 353-88.

Shiloh, M. U., MacMicking, J. D., Nicholson, S., Brause, J. E., Potter, S., Marino, M., Fang, F., Dinauer, M., and Nathan, C. 1999. Phenotype of mice and macrophages deficient in both phagocyte oxidase and inducible nitric oxide synthase. Immunity. 10: 29-38.

Soukup, J. M., Ghio, A. J., and Becker, S. 2000. Soluble components of Utah Valley particulate pollution alter alveolar macrophage function in vivo and in vitro. Inhal. Toxicol. 12: 401-414.

Steerenberg, P., Verlaan, A., De Klerk, A., Boere, A., Loveren, H., and Cassee, F. 2004. Sensitivity to ozone, diesel exhaust particles, and standardized ambient particulate matter in rats with Listeria monocytogenes-induced respiratory infection. Inhal. Toxicol. 16: 311-317.

Stoll, S., Jonuleit, H, Schmitt, E., Muller, G., Yarnauchi, H., Kurimoto, M., Knop, J., and Enk, A. H. 1998. Production of functional IL-18 by different subtypes of murine and human dendritic cells (DC): DC-derived IL-18 enhances IL-independent Th1 development. Eur. J. Immunol. 28:3231-3239.

Swain, S. L. 2001. Interleukin-18: Tipping the balance towards a T helper cell 1 response. J. Exp. Med. 194: F11-F14.

Trinchieri, G. 1995. Interleukin 12: A proinflammatory cytokine with immunoregulatory functions that bridge innate resistance and antigen-specific adaptive immunity. Annu. Rev. Immunol. 13: 251-276.

Tripp, C. S., Wolf, S. F. and Unanue, E. R. 1993. Interleukin 12 and tumor necrosis factor $\alpha$ are co-stimulators of interferon $\gamma$ production by natural killer cells in severe combined immunodeficiency mice with listeriosis, and interleukin 10 is a physiologic antagonist. Proc. Natl. Acad. Sci. USA. 90: 3725-3729. 
Tripp, C. S. Gately, M. K., Hakimi, J., Ling, P., and Unanue, E. R. 1994. Neutralization of IL-12 decreases resistance to Listeria in SCID and C.B-17 mice. Reversal by IFNgamma. J. Immunol. 152: 1883-1887.

Tsutsui, H., Nakanishi, K., Matsui, K., Higashino, K., Okamura, H., Miyazawa, Y., and Kaneda, K. 1996. IFN-gamma-inducing factor up-regulates Fas ligand-mediated cytotoxic activity of murine natural killer cell clones. J. Immunol. 157: 3967-3973.

Van der Veen, R. C., Dietlin, T. A., Pen, L., and Gray, J. D. 1999. Nitric oxide inhibits proliferation of T-helper 1 and 2 lymphocytes without reduction in cytokine secretion. Cell. Immunol. 193: 194-201.

Van der Veen, R. C., Dietlin, T. A., Gray, J. D., and Gilmore, W. 2000. Macrophagederived nitric oxide inhibits the proliferation of activated $\mathrm{T}$ helper cells and is induced during antigenic stimulation of resting T cells. Cell. Immunol. 199: 43-49.

Van Loveren, H., Rombout, P. J., Wagenaar, S. S., Walvoort, H. C., and Vos, J. G. 1988. Effects of ozone on the defense to a respiratory Listeria monocytogenes infection in the rat. Suppression of macrophage function and cellular immunity and aggravation of histopathology in lung and liver during infection. Toxicol. Appl. Pharmacol. 94: 374-393.

Van Zijverden, M., van der Pijl, A., Bol, M., van Pinxteren, F. A., de Haar, C., Penninks, A. H., van Loveren, H., and Pieters, R. 2000. Diesel exhaust, carbon black, and silica particles display distinct Th1/Th2 modulating activity. Toxicol. Appl. Pharmacol. 168: 131-139.

Yang, H-M., Antonini, J. M., Barger, M., W., Butterworth, L., Roberts J. R., Ma, J. K., Castranova, V., and Ma, J. Y. 2001. Diesel exhaust particles suppress macrophage function and slow pulmonary clearance of Listeria monocytogenes in rats. Environ. Health Perspect. 109: 515-521.

Yin, X. J., Schafer, R., Ma, J. Y., Antonini, J. M., Weissman, D. N., Siegel, P. D., Barger, M. W., Roberts, J. R., and Ma, J. K. H. 2002. Alteration of pulmonary immunity to Listeria monocytogenes by diesel exhaust particles (DEPs). I. Effects of DEPs on early pulmonary responses. Environ. Health Perspect. 110: 1105-1111.

Yin, X. J., Schafer, R., Ma, J. Y., Antonini, J. M., Roberts, J. R., Weissman, D. N., Siegel, P. D., and Ma, J. K. 2003. Alteration of pulmonary immunity to Listeria monocytogenes by diesel exhaust particles (DEPs). II. Effects of DEPs on T-cellmediated immune responses in rats. Environ. Health Perspect. 111: 524-530.

Yin, X. J., Dong, C. C., Ma, J. Y., Antonini, J. M., Roberts, J. R., Stanley, C. F., Schafer, R., and Ma, J. K. 2004a. Suppression of cell-mediated immune responses to listeria infection by repeated exposure to diesel exhaust particles in brown Norway rats. Toxicol. Sci. 77: 263-271. 
Yin, X. J., Ma, J. Y. C., Antonini, J. M., Castranova, V., and Ma, J. K. H. 2004b. Roles of reactive oxygen species and heme oxygenase- 1 in modulation of alveolar macrophagemediated pulmonary immune responses to Listeria monocytogenes by diesel exhaust particles. Toxicol. Sci. 82: 143-153.

Zelikoff, J. T., Schermerhorn, K. R., Fang, K., Cohen, M. D., and Schlesinger, R. B. 2002. A role for associated transition metals in the immunotoxicity of inhaled ambient particulate matter. Environ. Health Perspect. 110(Suppl. 5):871-875.

Zelikoff, J. T., Chen, L. C., Cohen, M. D., Fang, K., Gordon, T., Li, Y., Nadziejko, C., and Schlesinger, R. B. 2003. Effects of inhaled ambient particulate matter on pulmonary antimicrobial immune defense. Inhal. Toxicol. 15: 131-150. 
STUDY 3: SOLUBLE NICKEL, A COMPONENT OF RESIDUAL OIL FLY ASH, ALTERS PULMONARY HOST DEFENSE IN RATS 


\section{$\underline{\text { Abstract }}$}

Metals associated with air pollutants, particularly combustion-derived particulate matter, have been correlated with increased morbidity and mortality in humans. The soluble metal fraction of residual oil fly ash (ROFA) has been shown to alter the clearance of bacteria from the lungs of rats. In addition, various soluble metals present in ROFA have been associated with increased susceptibility in animal models. The goal of this study was to determine which of the primary soluble metals or combinations of metals in ROFA were responsible for increased susceptibility to infection and the potential mechanism responsible for the increased infectivity. The soluble fraction of ROFA contained $\mathrm{Ni}, \mathrm{Fe}, \mathrm{Al}$, and $\mathrm{Zn}$. Stock solutions of the metals were prepared in deionized water, and were further diluted to the instillate concentration in phosphatebuffered saline (PBS). On day 0, Sprague-Dawley rats were intratracheally instilled with $\mathrm{NiCl}_{2}(55.7 \mu \mathrm{g} / \mathrm{rat}), \mathrm{FeSO}_{4}(32.7 \mu \mathrm{g} / \mathrm{rat}), \mathrm{Al}_{3}\left(\mathrm{SO}_{4}\right)_{2}(46.6 \mu \mathrm{g} / \mathrm{rat})$, or $\mathrm{ZnCl}_{2}(8.69 \mu \mathrm{g} / \mathrm{rat})$, or a combination of all the metals at the concentration listed (Total Mixture group). At day $3,5 \times 10^{4}$ Listeria monocytogenes were intratracheally instilled into rats from each treatment group. At days 6, 8 and 10, left lungs were removed, homogenized, and cultured to assess bacterial clearance. Bronchoalveolar lavage (BAL) was performed on the right lungs on day 3 , prior to infection, and on days 6,8 and 10. A concentrated first fraction of lavage fluid was retained for analysis of lactate dehydrogenase (LDH), albumin, and reactive oxygen and nitrogen species as indices of lung damage and oxidative stress, and a variety of cytokines were measured to determine the activity of cells involved in the innate and adaptive immune responses in the lung. BAL cells were collected and cell differentials were performed to assess total number of alveolar macrophages (AMs), neutrophils, and lymphocytes that infiltrated the lungs. In addition, lymphocyte phenotype was analyzed by flow cytometry. In a separate set of experiments, rats were instilled with metal sulfate mixtures, including the total mixture, the mixture without $\mathrm{Fe}(\mathrm{Mix}-\mathrm{No} \mathrm{Fe}$ ), the mixture without $\mathrm{Ni}(\mathrm{Mix}-\mathrm{No} \mathrm{Ni}$ ), the mixture without $\mathrm{Al}$ (Mix - No Al), the mixture without Zn (Mix - No Zn), or PBS (vehicle control). At day 3, $5 \times 10^{4}$ Listeria monocytogenes were intratracheally instilled into rats from each treatment group and the same parameters were monitored in these groups of rats as described above. Prior to infection, soluble Ni and Total Mixture treatment increased LDH, albumin, and nitric oxide levels in the BAL, and phagocyte production of reactive oxygen species to a comparable degree, and were significantly elevated when compared to control. Metal combinations that included Ni, but not Ni alone, increased total BAL cell numbers (macrophages and neutrophils). Metal mixtures also decreased IL-10 prior to infection. Post-infection, rats instilled with soluble $\mathrm{Ni}$, alone or in combination with other metals, had decreased body weight and increased bacterial lung burden on day 6 , and body weight continued to decrease in the soluble Ni, Mix - No Fe, and Mix - No Al groups post-infection. Macrophage and neutrophil numbers were increased in all groups containing Ni post-infection. Lymphocyte numbers were elevated on day 10 in the groups that received soluble Ni alone, Mix - No Fe, or Mix - No Al. The increase in lymphocytes could be attributed to CD4+ and CD8+ T cells. BAL nitric oxide levels were increased post-infection on day 6 in all groups that contained $\mathrm{Ni}$, and production of reactive oxygen species by macrophages was increased in metal mixture groups that contained $\mathrm{Ni}$, but not 
in the group treated with $\mathrm{Ni}$ alone. IL-6 was elevated in all groups that contained $\mathrm{Ni}$ on day 6. IL-12 was increased on day 6, and IL-18 on days 8 and 10, only after treatment with $\mathrm{Ni}$ alone. $\mathrm{Ni}$, alone or in combination with other metals, significantly reduced IL-2 and IL- 4 on day 6 , and the reduction in IL- 2 continued in the rats that received Ni alone on days 8 and 10 after infection. To summarize, the metal mixture of $\mathrm{Al}, \mathrm{Fe}, \mathrm{Ni}$, and $\mathrm{Zn}$, induced lung injury and inflammation in a comparable fashion to that observed with the soluble portion of ROFA. The data indicate that the majority of inflammation and injury, prior to infection, and post-infection, was attributable primarily to $\mathrm{Ni}$. $\mathrm{Ni}$, alone or in combination with other metals, was the major determinant in increased susceptibility to infection. The elevated bacterial burden on day 6 in Ni-containing groups indicates that $\mathrm{Ni}$ is suppressing an early innate immune response. Ni alone activated cytokines involved in directing the $\mathrm{T}$ cell response to infection, which elicited the greatest influx of $\mathrm{T}$ cells into the lungs on day 10. However, Ni also appeared to be the most important factor in suppressing $\mathrm{T}$ cell activity once in the lung. Soluble $\mathrm{Ni}$, at the concentration measured in ROFA, is likely the primary metal involved in the increased susceptibility to infection observed in rats exposed to the soluble metals of ROFA. 


\section{Introduction}

Long term, large cohort epidemiological studies have correlated exposure to particulate matter (PM) with elevations in morbidity and mortality as indicated by increased hospital emissions for both cardiovascular and respiratory diseases (Abbey et al., 1999; Dockery et al., 1993; Englert, 2004; Ghio, 2004; Pope et al., 1995; 2004; Samet et al., 2000a; 2000b; Schwartz, 1994). Pulmonary effects attributed to PM exposure include, but are not limited to, increased airway reactivity and exacerbations in asthma, increased respiratory symptoms (cough, wheeze), decreased lung function, increased lung inflammation, and altered pulmonary host defense (American Thoracic Society, 1996). Pope et al. (2004) showed that the leading cause of increased morbidity and mortality in the category of pulmonary conditions was respiratory infection, primarily due to pneumonia and influenza.

Particle composition appears to be a strong determinant in PM toxicity. An epidemiological study by Burnett et al. (2000) showed that iron (Fe), sulfate $\left(\mathrm{SO}_{4}\right)$, nickel (Ni) and zinc $(\mathrm{Zn})$ in ambient $\mathrm{PM}_{2.5}$ correlated more strongly with mortality than $\mathrm{PM}_{2.5}$ mass, and Laden et al. (2000) were able to correlate particular metals in PM with various sources. In the latter study, the authors found that mortality had the closest correlation with fine particulates from oil combustion, followed by coal combustion, then mobile sources. PM, particularly in the fine particulate mode $\left(\mathrm{PM}_{2.5}\right)$, has been shown to alter immune parameters in humans. Population studies in industrialized areas have shown that, in regions with high anthropologic PM loads, whole blood and serum levels of lymphocytes, immunoglobulin (Ig) levels and types, and cytokines are significantly altered (Calderon-Garciduenas et al., 2000; Hertz-Picciotto et al., 2005; Leonardi et al., 
2000; Skachkova et al., 2001). Human exposure studies have also demonstrated that PM that is high in metals can alter cellular processes that are important in pulmonary responses to pathogens, including increases in the number and type of leukocytes in the lung, as well as elevations in oxidant and cytokine production by phagocytes (Ghio, 2004; Ghio et al., 2000; Schaumann et al., 2004).

Animal studies have shown that PM that contains metals, such as concentrated ambient air particulates (CAPs), residual oil fly ash (ROFA), copper smelter fly ash, and various forms of coal fly ash (CFA), can enhance susceptibility to infection or exacerbate preexisting pulmonary conditions (Antonini et al., 2002; Aranyi et al., 1981; Clarke et al., 1999; Hatch et al., 1985; Kodavanti et al., 2000; Zelikoff et al., 2003). Interestingly, in the study by Hatch et al. (1985), the investigators found that the ashes and particulates that were most potent at altering infectivity were the samples that were smallest in size and/or highest in soluble metal content. In addition, the various soluble metals that are associated with combustion-derived $\mathrm{PM}$, such as $\mathrm{Zn}, \mathrm{Fe}, \mathrm{Ni}$, and aluminum ( $\mathrm{Al}$ ), have been shown to alter susceptibility to pulmonary infection individually after inhalation or instillation into the lungs of rodents (Adkins et al., 1979; Drummond et al., 1986; Ehrlich, 1980; Ehrlich et al., 1978; Goldstein et al., 1979; Hatch et al., 1981; 1985; Sherwood et al., 1981; Zelikoff et al., 2002). Investigators appear to be in agreement on the data concerning the effects of soluble $\mathrm{Ni}$ and $\mathrm{Zn}$ on susceptibility to infection. However, there are discrepancies among studies that have examined infectivity after exposure to soluble Fe (Ehrlich, 1980; Goldstein et al., 1979; Hatch et al., 1981; Sherwood et al., 1981; Zelikoff et al., 2002) and soluble Al (Drummond et al., 1986; Ehrlich, 1980; Hatch et al., 1981). Discrepancies in the findings may be due to dose, route of exposure, and quantity 
and strain of bacteria used. In addition, there are relatively limited, if any, studies that examine the effects of respiratory exposure to the above metals in combination with each other on susceptibility to pulmonary infection.

Soluble metals are known to have a variety of effects on cells involved in pulmonary host defense, such as alveolar macrophages (AMs), neutrophils, natural killer (NK) cells, and lymphocytes. Numerous studies have been reviewed by Cohen (2004) regarding the effects of pulmonary exposure to soluble $\mathrm{Ni}, \mathrm{Al}$, or $\mathrm{Zn}$ on lung immune cells. Very little information is available on the effects of soluble $\mathrm{Al}$ on lymphocytes after pulmonary exposure; however, it has been shown to inhibit AM oxygen consumption and phagocytosis (Castranova et al., 1980a). The most common effects of $\mathrm{Zn}$ on the lung come from data concerning the insoluble oxide form of $\mathrm{Zn}$. Soluble $\mathrm{Zn}$ has also been shown to induce inflammation and injury in the lung, and inhibit AM phagocytosis; however, data on the effects of soluble $\mathrm{Zn}$ on lymphocytes in the lung is lacking (Cohen, 2004). Most of the information on soluble Ni comes from studies that use routes of exposure other than inhalation. However, there is strong evidence for the suppressive effects of soluble Ni on AMs, NK cells, and humoral immune responses (Smialowicz, 1998; Sunderman et al., 1989). Most of the studies that examine the effects of soluble Fe on immune cell function are dietary limitation or supplementation studies (Omara et al., 1998). These studies indicate that Fe deficiency or overload can have suppressive effects on the immune system. Respiratory exposure to soluble Fe has been shown to cause inflammation and cytoxicity in the lung (Dreher et al., 1997; Kodavanti et al., 1998), and can result in decreased lymphocyte proliferation systemically after respiratory exposure (Zelikoff et al., 2002). The data on AM function is more conflicting. Johansson et al. 
(1992) found that $\mathrm{FeCl}_{2}$ stimulated AM phagocytosis, whereas Skornik and Brain (1983) have demonstrated that exposure to $\mathrm{FeSO}_{4}$ appears to inhibit AM phagocytosis.

Combinations of these metals have also resulted in antagonistic effects. Soluble Fe has been found to reduce the production of pro-inflammatory cytokines by AMs induced by soluble Ni in culture (Salnikow et al., 2004), and to somewhat diminish the severe lung injury induced by soluble Ni in vivo (Dreher et al., 1997; Kodavanti et al., 1998).

Therefore, the metals present in the soluble fraction of ROFA may have variable effects on pulmonary immune cell function depending on which metals are present and in what concentration and form they exist.

In study 1 and 2, the soluble fraction of ROFA was shown to significantly decrease the pulmonary clearance of Listeria monocytogenes in rats. The soluble component of the ROFA used in those studies consisted primarily of $\mathrm{Al}, \mathrm{Fe}, \mathrm{Ni}$ and $\mathrm{Zn}$. It appeared that inhibition of AM phagocytosis and killing led to a higher bacterial burden early in the infection, and inhibition of, delay in, or alteration of the adaptive immune response resulted in the slowing of the bacterial clearance over time. The purpose of this study was to determine which metal or combination of metals, at the concentrations measured in the soluble fraction of ROFA, would best replicate the effects seen with the soluble ROFA sample. Based on the data available indicating the inhibitory effects of $\mathrm{Ni}$ on a variety of cells involved in pulmonary host defense and that $\mathrm{Ni}$ was one of the metals in highest concentration in this sample of ROFA, the hypothesis was that $\mathrm{Ni}$ would be one of the primary metals involved in the increase in susceptibility caused by the soluble fraction of ROFA. 
To investigate the hypothesis, a similar experimental design as study 2 was employed. Sprague-Dawley rats were given an intratracheal instillation (I.T.) of the soluble form of $\mathrm{Zn}, \mathrm{Fe}, \mathrm{Al}$, or $\mathrm{Ni}$ at the concentrations measured in the soluble ROFA sample, either individually, or in a mixture. In a separate group of experiments, rats were given an I. T. dose of the total metal mixture containing all four metals, or various combinations of three metals. Rats were intratracheally inoculated with $L$. monocytogenes 3 days after metal exposures, and bacterial clearance was monitored for up to one week post-infection. In addition, total numbers and phenotypes of bronchoalveolar lavage cells were monitored, and oxidant and cytokine production were measured to assess the overall immune response. 


\section{Methods - Part One}

Animals - Male Sprague-Dawley [Hla:(SD)CVF] rats (Hilltop Laboratories, Scottdale, PA) weighing 250-300 g, approximately 10 weeks old, were used for all experiments. They were given the ProLab 3500 diet and tap water ad libitum, housed in a clean air and viral- and antigen-free room with restricted access in an AAALACapproved animal facility, and allowed to acclimate for one week before use. The rats were monitored and found to be free of endogenous viral pathogens, parasites, mycoplasms, Helicobacter and CAR Bacillus.

Materials - L. monocytogenes (strain 10403S, serotype 1) was obtained as a gift from Dr. Rosana Schafer of the Department of Microbiology and Immunology at West Virginia University. Metal chlorides and sulfates were purchased from Sigma-Aldrich Co., St. Louis, MO.

Experimental Design - At day 0, animals were pre-exposed to individual metals, metal mixtures, or phosphate-buffered saline (vehicle control) by intratracheal instillation. At day 3, the animals were inoculated via intratracheal instillation with $5 \times 10^{4}$ L. monocytogenes. At days 6, 8, and 10, animals were euthanized and the left lungs of animals were clamped off, removed, homogenized, and the number of colony forming units (CFUs) was counted. The right lungs of animals were lavaged on days 3 (preinfection), 6, 8, and 10, and the cells and the fluid were retained for analysis as described as follows.

Metal Treatment: Individual Metals -Fe sulfate $\left(\mathrm{FeSO}_{4}\right)$, $\mathrm{Ni}$ chloride $\left(\mathrm{NiCl}_{2}\right)$, Al sulfate $\left(\mathrm{Al}_{2}\left(\mathrm{SO}_{4}\right)_{3}\right)$, or $\mathrm{Zn}$ chloride $\left(\mathrm{ZnCl}_{2}\right)$ were suspended in deionized water at a concentration of $10 \mathrm{mg} / \mathrm{ml}$. Individual metals were further diluted in sterile phosphate- 
buffered saline (PBS) to the concentrations listed in Table 1.2 for the soluble component of ROFA. There was no visible precipitate after dilution of the stock solutions in PBS. The artificial metal mixture was prepared using the same concentrations present in the soluble ROFA sample. The metal solutions were sonicated for 1 min with a Sonifier 450 Cell Disruptor (Branson Ultrasonics, Danbury, CT).

Rats were lightly anesthetized by an intraperitoneal injection of $0.6 \mathrm{ml}$ of a $1 \%$ solution of sodium methohexital (Brevital, Eli Lilly, Indianapolis, IN) and intratracheally instilled with the soluble metal solutions or sterile PBS (vehicle control) at a volume of $300 \mu \mathrm{l}$ per rat according to the method of Brain et al. (1976). The treatment groups were PBS (saline), iron (Soluble Fe, $32.7 \mu \mathrm{g} / \mathrm{rat}$ ), nickel (Soluble Ni, $55.7 \mu \mathrm{g} / \mathrm{rat}$ ), aluminum (Soluble Al, $46.6 \mu \mathrm{g} / \mathrm{rat}$ ), zinc (Soluble $\mathrm{Zn}, 8.69 \mu \mathrm{g} / \mathrm{rat}$ ), or a mixture of the metals in the quantities listed above (Total Mixture). There were 4 animals per group per time point.

Intratracheal Bacteria Inoculation - L. monocytogenes was cultured overnight in brain-heart infusion broth (Difco Laboratories, Detroit, MI) at $37^{\circ} \mathrm{C}$ in a shaking incubator. Following incubation, the bacterial concentration was determined spectrophotometrically at an optical density of $600 \mathrm{~nm}$. The sample was diluted to the concentration of $5 \times 10^{4}$ L. monocytogenes in $500 \mu$ of sterile PBS. The bacteria were intratracheally instilled 3 days post-metal sample instillation. The dose of $L$. monocytogenes was chosen based on the first study and was found to give a uniform infection and did not result in mortality in untreated naïve Sprague-Dawley rats in a previous study.

Morbidity/ Pulmonary Clearance of $L$. monocytogenes - Animal weights were monitored over the course of the treatment period as an indicator of morbidity. Rats were 
euthanized with an overdose of sodium pentobarbital, and at days 6,8 , and 10, the left lungs were removed from all the rats in each treatment group. The excised tissues were suspended in $10 \mathrm{ml}$ of sterile water, homogenized using a PowerGen 700 homogenizer (Fisher Scientific, Pittsburgh, PA), and cultured quantitatively on brain-heart infusion agar plates (Becton Dickinson and Co., Cockeysville, MD). The number of viable CFUs was counted after an overnight incubation at $37^{\circ} \mathrm{C}$.

Bronchoalveolar Lavage (BAL) - BAL was performed on days 3 (preinfection), 6, 8, and 10 on the right lungs of the rats from each treatment group as described in Study 2. The acellular BAL fluid (BALF) from the first lavage, and the BAL cells from the total lavage, including the first lavage, were retained for further analysis.

Analysis of Albumin and Lactate Dehydrogenase (LDH) - The presence of albumin and LDH in the BAL fluid was measured to evaluate the loss of integrity of the alveolar-capillary barrier and general cytotoxicity, respectively. Measurements of both albumin and LDH in the acellular fluid were obtained using a Cobas Mira analyzer (Roche Diagnostic Systems, Montclair, IN). Albumin was determined by spectrophotometric measurement of the reaction product of albumin with bromcresol green (628nm) according to a method by Sigma Diagnostics (St. Louis, MO). LDH activity was quantified by detection of the oxidation of lactate coupled to the reduction of $\mathrm{NAD}+$ at a spectrophotometric setting of $340 \mathrm{~nm}$ over time.

Pulmonary Cell Differentials and Phenotyping - Total BAL cells were counted using a Coulter Multisizer II (Coulter Electronics, Hialeah, FL). Cell differentials were performed to determine the total number of AMs, polymorphonuclear cells (PMNs), and lymphocytes as describe in Study 2. To determine phenotype of the lymphocyte 
population in the BAL, cells were labeled with fluorescently tagged antibodies against specific cell surface markers (BD Biosciences Pharmingen, San Diego, CA). The lymphocytes were identified by specific cell surface markers and were NK cells

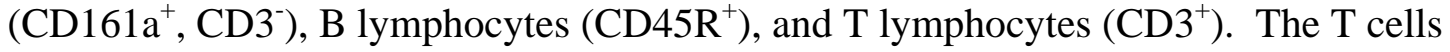
were further differentiated as T-helper $\left(\mathrm{T}_{\mathrm{H}}\right)$ cells $\left(\mathrm{CD} 4^{+}\right)$or cytotoxic $\mathrm{T}$ cells $\left(\mathrm{CD} 8^{+}\right)$. The lymphocyte population was characterized using a flow cytometer (FACS Calibur, BD Biosource, San Diego, CA), and the number of viable cells and total number of each lymphocyte was calculated as described in Study 2.

Measurement of Nitric Oxide - The presence of nitric oxide in acellular BAL was determined as the accumulation of nitrite using a modified microplate assay using the Greiss reagent (Green et al., 1982). Total nitrite was measured after a 1 hour incubation with a nitrate reductase to convert any nitrate in the samples to nitrite as described in Study 2. The measurement of total nitrite represents the presence of both nitrate and nitrite (NOx) in the samples.

\section{Luminol-Dependent Chemiluminescence (CL) - Luminol-dependent CL was}

performed on BAL cells as a measure of the light generated by the production of reactive oxygen species (ROS) by AMs and PMNs using a Berthold LB953 luminometer

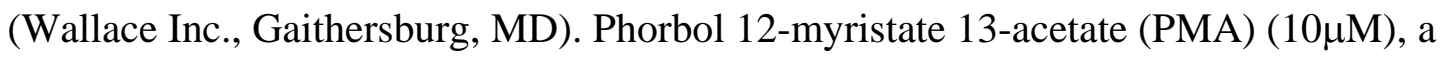
soluble stimulant of total BAL phagocytes (AMs and PMNs), and non-opsonized, insoluble zymosan (2 mg/ml), a stimulant of AMs only (Castranova et al., 1990), were added to the assay immediately prior to CL measurement to determine the contribution of both AMs and PMNs to the overall production of ROS in the lungs of the rats. Measurement of CL was recorded for 15 minutes at $37^{\circ} \mathrm{C}$, and the integral of counts per 
minute (cpm) versus time was calculated. CL was calculated as the cpm of the stimulated cells minus the cpm of the corresponding resting cells.

Cytokine Measurements in BAL - Cytokines present in the BAL fluid were analyzed by enzyme-linked immunosorbent assay (ELISA) using commercially available ELISA kits (BioSource International Inc., Camarillo, CA) to determine activity of various cell types involved in the immune response. The following cytokines were quantified: tumor necrosis factor- $\alpha$ (TNF- $\alpha$ ), interleukin (IL)-2, IL-4, IL-6, IL-10, IL-12p70, IL-18, and interferon- $\gamma(\mathrm{IFN}-\gamma)$.

Statistical Analysis - Results are expressed as means \pm standard errors of measurements (SE). Statistical analyses were carried out with the SigmaStat 3.1 statistical program (Chicago, IL). The significance of the interaction among different treatment groups for the different parameters at each time point was assessed using analysis of variance (ANOVA). The significance of difference between individual groups was analyzed using the Student-Newman-Keuls method with the criterion of significance set at $p<0.05$. To further distinguish part one from part two of study, significance will be indicated by symbols in part one and by lower case letters in part two. 


\section{Methods - Part Two}

Metal Treatment: Metal Combinations - Fe sulfate $\left(\mathrm{FeSO}_{4}\right)$, Ni sulfate $\left(\mathrm{NiSO}_{4}\right), \mathrm{Al}$ sulfate $\left(\mathrm{Al}_{2}\left(\mathrm{SO}_{4}\right)_{3}\right)$, or $\mathrm{Zn}$ sulfate $\left(\mathrm{ZnSO}_{4}\right)$ were suspended in deionized water at a concentration of $10 \mathrm{mg} / \mathrm{ml}$. Metal mixtures were further diluted in sterile phosphate-buffered saline (PBS) to the concentrations listed in Table 1.2 for the soluble component of ROFA. The total artificial metal mixture was prepared using the same concentrations present in the soluble ROFA sample. The metal solutions were sonicated for 1 min with a Sonifier 450 Cell Disruptor (Branson Ultrasonics, Danbury, CT).

Rats were lightly anesthetized by an intraperitoneal injection of $0.6 \mathrm{ml}$ of a $1 \%$ solution of sodium methohexital (Brevital, Eli Lilly, Indianapolis, IN) and intratracheally instilled with the soluble metal solutions or sterile PBS (vehicle control) at a volume of $300 \mu \mathrm{l}$ per rat according to the method of Brain et al. (1976). The treatment groups were PBS (Saline), the total mixture without soluble iron (Mix - No Fe), the total mixture without soluble nickel (Mix - No Ni), the total mixture without soluble aluminum (Mix No Al), the total mixture without soluble zinc (Mix - No Zn), or the total mixture of the soluble metals (Total Mixture) in the quantities present in the soluble ROFA fraction detailed in part 1 of the study. Table 3.2.1 lists all quantities of the soluble metals used for all treatment groups. There were 8 animals per group per time point. The animals, experimental design, assays, and statistical analyses were identical to that of Part 1 of the study. Significance in part two will be indicated by lower case letters. 


\section{Results - Part One}

Morbidity and Pulmonary Bacterial Clearance - Morbidity in animals pretreated with Saline, Soluble Fe, Soluble Ni, Soluble Al, Soluble Zn, or the Total Mixture was monitored as the $\%$ change in body weight after infection with L. monocytogenes (Figure 3.1.1). Body weights of animals from all treatment groups increased prior to infection and the $\%$ change in body weight did not differ significantly among groups from day 0 to day 3 (data not shown). Rats pretreated with Soluble Ni or the Total Mixture lost significantly more weight post-infection when compared to the saline control group as well as the soluble Fe, Al, and Zn groups. Soluble Fe, Soluble Al, and Soluble Zn, did not differ from Saline-treated rats. Rats in all of the treatment groups, except the Soluble Ni group began to regain body weight by day 8 . In contrast, the significant decrease in the \% change in body weight of the animals treated with Soluble Ni prior to infection continued until day 10.

Animals treated with the individual metals were able to clear the majority of bacteria from the lungs by day 10 (Table 3.1.1), and bacterial burden in the left lungs did not differ significantly at any time point post-infection among these groups. However, rats pre-exposed to Soluble Ni or the Total Mixture had a significantly greater bacterial lung burden on day 6 when compared to all other groups. The increase in CFUs in the lungs of the Soluble Ni group continued on day 8 although the increase in bacteria was not significant at this time point. By day 10 there was no significant difference in bacterial lung burdens between treatment groups.

Biochemical Analysis of BAL Fluid: LDH and Albumin - The presence of the intracellular enzyme, $\mathrm{LDH}$, in the BAL fluid of rats was used as a marker for general 
cellular cytotoxicity (Figure 3.1.2A). LDH was significantly elevated in the Soluble Ni and Total Mixture groups at day 3 (prior to infection) and on days 6 and 8 when compared to all other groups, but did not differ from each other. LDH levels of animals pre-treated with Saline, Soluble Fe, Soluble Al, or Soluble Zn, did not differ.

Albumin in the BAL fluid, an indicator of the breakdown of the alveolar blood-air barrier, was increased in Soluble Ni and Total Mixture groups on day 3, pre-infection as compared to animals treated with Saline, Soluble Fe, Soluble Al, or Soluble Zn. (Figure 3.1.2B). After infection with the bacteria on day 3, the increase in albumin persisted in the Total Mixture group until day 6. In the Soluble Ni group, albumin levels were elevated when compared to all groups except the Total Mixture throughout the time course.

BAL Cellular Profiles - Total BAL cell number (Figure 3.1.3A) was counted and the total number of AMs (Figure 3.1.3B), PMNs (Figure 3.1.3C), and lymphocytes (Figure 3.1.3D) were calculated from cell differentials. On day 3, prior to infection, rats pre-exposed to the Total Mixture had a significantly greater number of PMNs when compared to the all other groups except Soluble $\mathrm{Ni}$, indicating an enhanced inflammatory response in this group. Post-infection, there was a general increase in the total number of BAL cells in all groups with a significant increase in the animals pre-treated with Soluble Ni or Total Mixture on days 6, 8, and 10 when compared to Saline, Soluble Fe, Soluble $\mathrm{Al}$, or Soluble $\mathrm{Zn}$. The increases at the early time points post-infection are due to the influx of neutrophils and some AMs (Figure 3.1.3B and C), whereas at the later time points after infection the increase appears to be due to AMs (Figure 3.1.3B). In the case 
of the Soluble Ni group, lymphocyte numbers increased on day 10 when compared to lymphocyte influx in all other groups (Figure 3.1.3D).

Flow cytometry, in conjunction with cell differential counts, was used to determine total number of NK cells (Figure 3.1.4A), total T cells (Figure 3.1.4B), CD4+ T cells (Figure 3.1.4C), and CD8+ T cells (Figure 3.1.4D). On day 3, prior to infection, there were significant increases in the NK cell population in the Total Mixture group when compared to the Saline, Soluble Fe, and Soluble Al groups, and a significant increase in total $\mathrm{T}$ cell population in the Total Mixture group when compared to all other treatments. After inoculation with L. monocytogenes, there was a general increase in lymphocytes over the course of the infection in all groups. However, the most significant increase was in the T cell population on day 10 of the rats pre-treated with Soluble Ni. This increase was attributable to both the CD4+ and CD8+ T cell subsets.

On day 3 , before infection, there were no significant differences among the groups in the T/B cell ratio (Table 3.1.2) or the CD4+/CD8+ T cell ratio (Table 3.1.3), although the CD4+/CD8+ T cell ratio appeared to be slightly less in the Soluble Ni and Soluble $\mathrm{Zn}$ groups when compared to the Saline group. Over the course of the infection, there was a continual increase in the T/B cell ratio in all groups with the highest ratio on day 10 , reflecting the dominance of the cell-mediated immune system in the later, adaptive immune response to $L$. monocytogenes. The highest T/B cell ratio appeared in the Soluble Ni group on day 10; however, it was not significantly different than that of the other groups. On day 8 , there was a significant decrease in the number of B cells in the R-Soluble group when compared to the saline group reflected as a slightly higher T/B cell ratio at this time point. In most groups the CD4+/CD8+ T cell ratio decreased or 
remained the same post-infection; however, there were no significant differences among treatments.

BAL Fluid Levels of Nitric Oxide - The presence of reactive nitrogen species, known antimicrobial agents, as well as an indicator for increased oxidative stress, was measured as total nitrite (NOx) (Figure 3.1.5). BAL NOx levels were elevated in the Soluble Ni and Total Mixture groups on day 3, prior to infection, when compared to all other groups. As would be expected in response to L. monocytogenes infection, BAL NOx levels increased approximately 2-fold in the Saline, Soluble Fe, Soluble Al, and Soluble Zn groups post-infection, when compared to values on day 3. However, there was a 3.5-fold increase in the BAL NOx in the Soluble Ni and Total Mixture groups on day 6 , when compared to day 3 , and these two groups were significantly elevated when compared to all other groups.

\section{Oxidative Potential of Phagocytes: PMA- and zymosan-stimulated CL -}

Luminol-dependent CL was performed on BAL cells to determine the level of production of ROS by AMs and PMNs. On day 3 (pre-infection), after stimulation with PMA, a stimulant of both PMNs and AMs, there was a significant increase in CL in the soluble Ni and Total Mixture groups (Figure 3.1.6A). Post-infection, there was a general increase in $\mathrm{CL}$ in all groups at day 6 , which gradually decreased over the course on the infection. However, there were no significant differences among groups. AM production of ROS after stimulation with zymosan was increased in the Total Mixture group prior to infection on day 3 when compared to all groups except the Soluble Ni-treated rats (Figure 3.1.6B). There was a general increase in zymosan-stimulated CL after infection on day 6. However, there were no significant differences at this time point. On day 8, CL decreased 
in all groups; however, the Total Mixture group was significantly elevated when compared to all other groups.

BAL Cytokine Analysis - Cytokines present in the BAL were measured as indicators of cell specific activity in response to the soluble metals in ROFA followed by L. monocytogenes infection. TNF- $\alpha$ (Figure 3.1.7A) and IL-6 (Figure 3.1.7B) were measured in the BAL as indicators of the degree of the inflammatory response. There was no significant difference in TNF- $\alpha$ levels among treatment groups on day 3, prior to infection (Figure 3.1.7A). After infection, there was a general increase in TNF- $\alpha$ on day 6 in response to the bacteria. Although there were no significant differences at this time point, there was a slightly lower level of TNF- $\alpha$ in the BAL of rats pre-treated with Soluble Ni or the Total Mixture. There were no significant differences in TNF- $\alpha$ on days 8 or 10. IL-6, a pleiotropic cytokine, is considered to be pro-inflammatory and is indicative of the acute phase response, the reaction following insult or injury that results in fever, an increase in circulating leukocytes (particularly neutrophils) and an increase in phagocyte activity (Hirano et al., 1990). On day 3, pre-infection, there were no significant differences in the BAL level of IL-6 among the treatment groups (Figure 3.1.7B). Postinfection, on day 6, there was a significant elevation in IL-6 in the animals pre-treated with Soluble Ni or the Total Mixture when compared to all other treatments, indicating a more persistent and severe inflammatory response. The Saline, Soluble Al, Soluble Fe, and Soluble Zn groups did not differ from each other.

IL-10 has diverse effects on different cell types, but is considered to primarily be inhibitory in nature, and to be an anti-inflammatory cytokine. It is known to inhibit activation of $\mathrm{CD} 4+\mathrm{T}_{\mathrm{H}} 1$ and $\mathrm{CD} 8+\mathrm{T}$ cells, monocytes, macrophages and neutrophils, and 
have varying effects on NK cells depending on the presence of other cytokines (Moore et al., 2001), functions that are necessary in the cell-mediated immune response $L$. monocytogenes. On day 3 , prior to infection, there was a significant decrease in IL-10 in the Total Mixture group when compared to all other groups, indicating a possible decrease in the inhibitory controls on AMs in this group (Figure 3.1.8). After infection, there were no differences among groups on days 6 and 8. However, on day 10 there was a significant decrease in IL-10 in the Soluble Ni group when compared to all other treatments.

IL-12 and IL-18 are two cytokines known to induce the expression of IFN- $\gamma$ by CD4+ $T_{H} 1$ cells, CD8+ T cells, and NK cells. The presence of IL-12 and IFN- $\gamma$, in turn, drives the differentiation of naïve $\mathrm{CD} 4+\mathrm{T}$ cells toward the $\mathrm{CD} 4+\mathrm{T}_{\mathrm{H}} 1$ subset (Billiau, 1996; Mosmann and Sad, 1996; Paul and Seder, 1994). There were no significant differences between groups in the levels of IL-12p70 (the active form of IL-12) (Figure 3.1.9A) or IL-18 (Figure 3.1.9B) prior to infection. IL-12, secreted primarily by innate immune responders, should have a greater impact on the immune response in the early stages of infection as opposed to the later phase. After infection, there was an increase in IL-12 on day 6 in all groups with that of the Soluble Ni group being significantly elevated when compared to all other groups (Figure 3.1.9A). IL-18 increased in all groups postinfection, and there a was a significant increase in the Soluble Ni rats on days 8 and 10 when compared to all other groups (Figure 3.1.9B).

To determine the potential activity of the CD4+ T cell subsets in the lung, levels of IL-2 and IL-4 were measured in the BAL fluid. IL-2 is produced primarily by CD4+ $\mathrm{T}_{\mathrm{H}} 1$ cells and by naïve $\mathrm{CD} 4+$ and some $\mathrm{CD} 8+\mathrm{T}$ cells, whereas IL-4 is secreted mainly by 
the CD4 $+\mathrm{T}_{\mathrm{H}} 2$ subset (Mosmann and Sad, 1996). Levels of IL-2 (Figure 3.1.10A) and IL-4 (Figure 3.1.10B) did not differ among treatments groups on day 3, prior to infection. However, after infection, the was a significant decrease in BAL IL-2 in the Total Mixture group on day 6, and the Soluble Ni group at all time points post-infection. After infection on day 6 , there was also significantly less IL-4 in the BAL of rats pre-treated with Soluble Ni or the Total Mixture, when compared to all other groups. This reduction was still present in the Soluble Ni group on day 8. Levels of IL-2 and IL-4 did not differ between the Saline, Soluble Fe, Soluble Al, and Soluble Zn groups at any of the time points studied.

IFN- $\gamma$ is also produced by $\mathrm{CD} 4+\mathrm{T}_{\mathrm{H}} 1$ cells, as well as by $\mathrm{CD} 8+\mathrm{T}$ cells and $\mathrm{NK}$ cells. There were no significant differences prior to infection between groups, and there was a general increase at the first time point post-infection. However, similar to the results of Study 2, levels of this cytokine in the BAL were extremely variable across all time points, as well as within groups at a single time point (data not shown). Differences in IFN- $\gamma$ levels may be better detected at an earlier time point post-inoculation, or as it is produced by lymphocytes cultured directly from the tracheobronchial lymph nodes, rather than in the acellular BAL fluid. 


\section{$\underline{\text { Results - Part Two }}$}

Morbidity and Pulmonary Bacterial Clearance - Morbidity in animals pretreated with Saline, Mix - No Fe, Mix - No Ni, Mix - No Al, Mix - No Zn, or the Total Mixture was monitored as the \% change in body weight after infection with $L$. monocytogenes (Figure 3.2.1). Body weights of animals from all treatment groups increased prior to infection and the $\%$ change in body weight did not differ significantly among groups from day 0 to day 3 (data not shown). After infection, on day 6 there was a significant decrease in the \% change in body weight in animals pretreated with all metal mixtures except the mixture that lacked soluble Ni when compared to the Saline group. At day 8 , body weight of animals in the Mix - No Al group and the Mix - No Fe group remained significantly less than the Saline and Mix - No Ni groups, where the Mix - No Al group continued to lose weight and the Mix - No Fe group did not lose or gain in $\%$ body weight. Animals in the remaining groups all began to gain weight at varying degrees on day 8 . By day 10, animals pre-treated with Mix - No Al, and the Total Mixture still weighed significantly less than animals in the Saline and Mix - No Ni groups, and rat pre-treated with Mix - No Fe weighed less than the Mix - No Ni group. Animals from the Mix - No Zn group did not differ from the Saline or Mix - No Ni group on day 10. Throughout the time course, the rats from the Mix - No Ni group gained weight in an identical fashion to that of the rats in the Saline group.

Animals treated with Saline or Mix - No Ni also followed a similar bacterial clearance pattern, and did not differ significantly from each other at any time point (Table 3.2.2). However, rats pre-exposed to all the mixtures containing $\mathrm{Ni}$ (Mix - No Fe, Mix No Al, Mix -No Zn, and Total Mixture) had a significantly greater bacterial lung burden 
on day 6 when compared to the Saline and Mix - No Ni groups. At day 8, the rats pretreated with Mix - No Al had the highest bacterial burden, 10-fold higher than that of the Saline, Mix - No Ni, and Total Mixture groups; however, the difference was not significant. By day 10 there was no significant difference in bacterial lung burdens between treatment groups.

Biochemical Analysis of BAL Fluid: LDH and Albumin - The presence of the intracellular enzyme, $\mathrm{LDH}$, in the BAL fluid of rats was used a marker for general cellular cytotoxicity (Figure 3.2.2A), and the presence of albumin in the BAL served as an indicator of the breakdown of the air-blood barrier (Figure 3.2.2B). On day 3, prior to infection, LDH and albumin were elevated in all groups that contained soluble Ni (Mix No Fe, Mix - No Al, Mix -No Zn, and Total Mixture) when compared to rats treated with Saline or Mix - No Ni. LDH and albumin levels followed a similar pattern postinfection. On days 6 and 8, there were significant increases in these parameters in the groups that contained Ni when compared to the Saline and Mix - No Ni groups. There were no significant differences among groups on day 10 .

BAL Cellular Profiles - Total BAL cell number (Figure 3.2.3A) was counted and the total number of AMs (Figure 3.2.3B), PMNs (Figure 3.2.3C), and lymphocytes (Figure 3.2.3D) were calculated from cell differentials. On day 3, prior to infection, rats pre-exposed to Mix - No Fe, Mix - No Zn, or the Total Mixture had an increase influx of cells into the lungs when compare to the Saline and Mix - No Ni groups (Figure 3.2.3A). Post-infection, there was a general increase in the total number of BAL cells in all groups due primarily to AM and PMN influx early after infection and to AM and lymphocytes responses at the later time points post-infection. At all time points post- 
infection there were significant increases in the AM numbers of all group containing $\mathrm{Ni}$ when compared to Saline- and Mix - No Ni- treated rats (Figure 3.2.3B). Increases in total cell number were also attributed to significant influx of PMNs in all the groups containing soluble Ni on days 6 and 8 when compared to Saline and Mix - No Ni groups (Figure 3.2.3C). On day 10 there were significantly more lymphocytes present in the BAL of rats pre-treated with Mix - No Fe, Mix - No Al, and Total Mixture when compared to the Saline and Mix - No Ni groups (Figure 3.2.3D).

Flow cytometry, in conjunction with cell differential counts, was used to determine total number of NK cells (Figure 3.2.4A), total T cells (Figure 3.2.4B), CD4+ T cells (Figure 3.2.4C), and CD8+ T cells (Figure 3.2.4D). On day 3, prior to infection, there were slight, but significant, increases in the NK cell and the CD8+ T cell population in the Mix - No Zn and Total Mixture groups when compared the Saline-treated rats. After bacterial inoculation, all measured lymphocyte types increased over the course of the infection. NK cells increased approximately 2 -fold in all metal mixture groups; however, the increase was still less than that observed in the saline group. The highest levels of NK cells occurred on day 10 and there were no significant differences at this time point. $\mathrm{T}$ cell populations were also at the highest levels on day 10 , and there more $\mathrm{CD} 8+\mathrm{T}$ cells in groups that contained $\mathrm{Ni}$, with significant elevation in the Mix - No Fe and Mix-No Al groups, when compared to the Mix - No Ni group.

On day 3 , before infection, there were no significant differences among the groups in the T/B cell ratio (Table 3.2.3) or the $\mathrm{CD} 4+/ \mathrm{CD} 8+\mathrm{T}$ cell ratio (Table 3.2.4). Post-infection, there is initially a slight decrease in the T/B cell ratio (day 6) followed by a general increase in the ratio over the course of the infection, which is expected in a cell- 
mediated immune response. The increase in the T/B ratio on day 8 is greater in all the groups containing $\mathrm{Ni}$; however, the difference was not significant when compared to Saline and Mix - No Ni groups. In response to L. monocytogenes infection, the CD4+ and CD8+ T cell populations increase and a somewhat greater increase in CD8+ cells is expected, resulting in a decrease in the CD4+/CD8+ T cell ratio post-infection. In all treatment groups in this study, the CD4+/CD8+ T cell ratio decreased post-infection. However, there were no significant differences among treatments.

BAL Fluid Levels of Nitric Oxide - The presence of reactive nitrogen species, known antimicrobial agents, as well as an indicator for increased oxidative stress, was measured as total nitrite (NOx) (Figure 3.2.5). BAL NOx levels were elevated in the groups containing soluble $\mathrm{Ni}$ on day 3, prior to infection, and the Total Mixture levels were significantly different from the Mix - No Ni group. After infection, BAL NOx levels were significantly increased in the Mix - No Fe, Mix - No Al, Mix - No Zn, and Total Mixture groups on day 6. There were no significant differences among groups on days 8 and 10 .

\section{Oxidative Potential of Phagocytes: PMA- and zymosan-stimulated CL -}

Luminol-dependent CL was performed on BAL cells to determine the level of production of ROS by AMs and PMNs. Prior to infection, on day 3, there was an increase in the production of ROS by phagocytes stimulated with PMA in the Mix - No Zn and Total Mixture groups (Figure 3.2.6A). Post-infection there was an overall increase in PMAstimulated CL on day 6; however, there were no significant differences among treatments. AM production of ROS after stimulation with zymosan was increased in the Mix - No Zn and Total Mixture groups prior to infection on day 3 when compared to 
Saline-treated rats (Figure 3.2.6B). There was a general increase in zymosan-stimulated CL after infection on day 6 , and a significantly greater increase in the groups containing soluble Ni when compared to Saline and/or the Mix - No Ni groups. The rats pre-treated with Mix - No Al increased significantly when compared to all groups on day 6. No significant differences were observed in either PMA- or zymosan-stimulated CL on days 8 and 10.

BAL Cytokine Analysis- Cytokines present in the BAL were measured as indicators of cell specific activity in response to the various mixtures of soluble metals in ROFA followed by L. monocytogenes infection. The pro-inflammatory cytokines, TNF$\alpha$ (Figure 3.2.7A) and IL-6 (Figure 3.2.7B) were measured. There was no significant difference in TNF- $\alpha$ or IL-6 levels among treatment groups on day 3, prior to infection. After infection, there was a general increase in TNF- $\alpha$ on day 6 in response to the bacteria; however, there was less of an increase in the rats pre-treated with the Total Mixture when compared to the Saline-treated rats. There were no significant differences in TNF- $\alpha$ between groups on days 8 and 10. Post-infection, on day 6 , there was a significant elevation in IL-6 in the Mix - No Al and Mix - No Zn groups when compared to the Saline and Mix - No Ni groups, which did not differ from each other. Also, there was a significant increase in the Mix - No Fe group when compared to the Mix - No Ni groups at this time point. IL-6 levels diminish on days 8 and 10 with the only significant difference being a slight increase in the Mix - No Zn group on day 8 when compared to the Mix - No Ni group.

As discussed in part one of the current study, IL-10 has diverse effects on different cell types, but is considered to primarily be inhibitory in nature, and to be an 
anti-inflammatory cytokine. On day 3, prior to infection, all metal mixture groups showed significantly lower levels of IL-10 when compared to the Saline group (Figure 3.2.8). After infection, there were no differences among groups on days 6,8 , or 10 . Also described in part one, IL-12 and IL-18 are two cytokines that are intricately involved in directing the adaptive immune response toward a cell-mediated response (Billiau, 1996; Mosmann and Sad, 1996; Paul and Seder, 1994). There were no significant differences among groups in the levels of IL-12p70 (the active form of IL-12) (Figure 3.2.9A) or IL-18 (Figure 3.2.9B) prior to infection. As expected, IL-12 was elevated early after infection on day 6 in all groups, although to a lesser extent in the Mix- No Ni group. IL-18 increased in all groups post-infection with the highest levels occurring on day 8. Although there were no statistically significant changes, the groups containing soluble Ni tended to have a greater IL-18 response on day 8 compared to the Saline and Mix- No Ni groups (Figure 3.2.9B).

To determine the potential activity of the CD4+ T cell subsets in the lung, levels of IL-2 and IL-4 were measured in the BAL fluid. Levels of IL-2 (Figure 3.2.10A) and IL-4 (Figure 3.2.10B) did not differ among treatments groups on day 3, prior to infection. However, after infection, there were significant decreases in BAL IL-2 and IL-4 in all groups containing soluble Ni when compared to the Saline and Mix - No Ni groups. There was also a significant decrease in IL-2 and IL-4 in the Mix - No Fe group on day 8 when compared to the Mix - No Ni group, and a decrease in IL-4 in the Mix - No Al group on day 8 relative to the Mix - No Ni group as well. No significant differences were observed on day 10 . 
IFN- $\gamma$ is also important in the response of NK cells, T cells, and AMs. As similarly described in part 1 of the study, there was a general increase in IFN- $\gamma$ in all groups post-infection. However, there was a high level of variation among rats within a treatment group as well as between treatment groups (data not shown). Therefore, it is difficult to make any conclusions concerning this cytokine in this study. 


\section{$\underline{\text { Discussion }}$}

The purpose of this study was to determine which soluble metal or metal combination was responsible for the alterations in pulmonary host defense and increased susceptibility observed after exposure to the soluble ROFA sample used in Study 1 and 2. I.T. of the artificial metal mixture used in both parts of study 3 resulted in decreased pulmonary clearance of bacteria, increased lung injury and inflammation, and altered cellular profiles in a similar fashion, although not to the same degree, as was observed with the soluble fraction of ROFA in Study 2. Therefore, the four metals used in the artificial mixture (Al, Fe, $\mathrm{Ni}$, and $\mathrm{Zn}$ ) could account for the majority of the effects observed with the soluble ROFA sample. The major findings of this study are that, primarily soluble $\mathrm{Ni}$, alone or in combination with soluble $\mathrm{Fe}, \mathrm{Zn}$, or $\mathrm{Al}$, at the concentrations present in the soluble sample of ROFA (Table 3.2.1), accounted entirely for the increased susceptibility to infection. Soluble Ni had the greatest effect on lung injury and inflammation, similar to that measured in rats treated with the soluble ROFA sample, as indicated by increased LDH, albumin, macrophage, and neutrophil numbers in the BAL. The increased levels of BAL NOx and IL-6 in all groups that contained Ni were also comparable to that observed in study 2 in rats treated with the soluble fraction of ROFA. Lymphocyte profiles in rats pre-treated with soluble $\mathrm{Ni}$, alone or in combination with other metals followed the same pattern as the soluble ROFA sample in study 2, with increased $\mathrm{T}$ cells infiltrating the lungs on day 10 . In addition, a similar reduction in $\mathrm{T}$ cell cytokine production (IL-2 and IL-4) was observed in groups that were treated with soluble $\mathrm{Ni}$, indicating that the adverse effects on the adaptive immune response observed 
after treatment with soluble ROFA could be attributed, at least in part, to the soluble $\mathrm{Ni}$ content.

Prior to infection, rats in groups that were treated soluble Ni alone or in a metal mixture showed increased lung injury (Figure 3.1.2 \& 3.2.2) and inflammation (Fig 3.1.3 \& 3.2.3), as well as increased ROS (Figure 3.1.6 \& 3.2.6) and NO (Figure 3.1.5 \& 3.2.5) in BALF, particularly in the Total Mixture group. In addition, BALF IL-10 (Figure 3.1.8 \& 3.2.8) (an anti-inflammatory cytokine) was lower in the Total Mixture group in part one when compared to all of the individual metals or control. All the metal mixtures in part two of the study had lower IL-10 levels when compared to control. The increased injury and lung inflammation corresponded well with the presence of soluble $\mathrm{Ni}$ (alone or in a mixture), whereas the increase in oxidant production and decrease in antiinflammatory cytokine, IL-10, coincided better with mixtures of metals, particularly the Total Mixture group. Treatment with soluble Ni alone did result in increased NO production and increased CL in response to PMA prior to infection. In addition, there was a slight increase in NO production, but not significant, in all the groups that contained $\mathrm{Ni}$; however, the elevations were not as great as that observed in the Total Mixture group. The findings concerning the role of $\mathrm{Ni}$ in injury and lung inflammation are consistent with that of Dreher et al. (1997) and Kodavanti et al. (1997; 1998), where the investigators found that soluble $\mathrm{Ni}$ at concentrations present in ROFA produced a more severe inflammation than when in combination with soluble Fe or V. Interestingly, Kodavanti et al. (1998) found that oxidant production correlated best with the soluble V content in their ROFA sample. In the ROFA sample from studies 1 and 2, there was very little $\mathrm{V}$ present in the soluble fraction. Therefore, soluble $\mathrm{V}$ was not tested in this study. 
Instead, oxidant production in the present study correlated best with total metal content, and $\mathrm{Ni}$ alone had some potential for inducing increased ROS and NO, although not to the degree of the Total Metal mixture. It is possible that the existing inflammation and excess injury in the groups that contained soluble Ni may have contributed to the increase in susceptibility to infection in the groups that contained soluble $\mathrm{Ni}$.

Bacterial clearance from the lungs of rats pre-treated with the Total Mixture was significantly reduced on day 6 when compared to all other groups that did not contain $\mathrm{Ni}$, and body weight was also significantly reduced at the early time point post-infection in this group as well (Table 3.1.1 \& 3.2.2). This result was duplicated only with Soluble Ni alone, or in combination with the other metals. Neither soluble Fe, $\mathrm{Zn}$, nor $\mathrm{Al}$ alone significantly altered bacterial clearance from the lungs of rats at the concentrations used in this study. Interestingly, in part one of the study, rats treated with $\mathrm{Ni}$ alone appeared to have a more severe infection than all other groups, including the Total Mixture, as indicated by significant loss of body weight (Figure 3.1.1 \& 3.2.1). Also, in part two, the metal combination that produced the greatest increase in susceptibility to infection was the group that contained $\mathrm{Ni}$, but lacked $\mathrm{Al}$, indicating that $\mathrm{Al}$ may act in an antagonistic fashion to soluble Ni. The group with the second worst infection lacked Fe, and rats treated with Fe alone had the fastest clearance rate on day 6; therefore, soluble Fe may also act antagonistically to $\mathrm{Ni}$. Although the difference was not significant, the animals pre-treated with soluble $\mathrm{Zn}$ alone, did have slightly more bacteria in the lungs on day 6 than the groups treated with soluble Fe or Al. The concentration of soluble $\mathrm{Zn}$ used in the study was relative low when compared to $\mathrm{Ni}, \mathrm{Fe}$, and $\mathrm{Al}$, at approximately $1 / 5^{\text {th }}$ that of 
the other metals. It is still possible that, in samples that contain higher levels of soluble Zn, Zn may have adverse effects on pulmonary host defenses.

Other studies that use higher levels of Zn have found it to enhance susceptibility to infection in rodents. Exposure to $\mathrm{ZnSO}_{4}$ in mice either by I.T. or inhalation, prior to infection with Streptococcus pyogenes, increased infectivity and resulted in enhanced mortality in mice (Ehrlich, 1980; Ehrlich et al., 1978; Hatch et al., 1981). Other investigators that have examined the effects of soluble Ni on infectivity are in agreement with the findings of this study. Adkins et al. (1979) found that inhalation of approximately $500 \mu \mathrm{g} / \mathrm{m}^{3}$ of $\mathrm{Ni}$ chloride $\left(\mathrm{NiCl}_{2}\right)$ or $\mathrm{Ni}$ sulfate $\left(\mathrm{NiSO}_{4}\right)$ enhanced mortality in mice infected with S. pyogenes. Zelikoff et al. (2002) showed that inhalation of $65-90 \mu \mathrm{g} / \mathrm{m}^{3}$ of $\mathrm{Ni}$ or $\mathrm{Fe}^{2+}$ as chlorides $\left(\mathrm{NiCl}_{2}\right.$ or $\left.\mathrm{FeCl}_{2}\right)$ resulted in increased infectivity in rats that had been previously infected with an I.T. of Streptococcus pneumoniae. In the study by Zelikoff et al. (2002), inhalation of $\mathrm{NiCl}_{2}$ was found to inhibit the clearance of the bacteria from the lungs, whereas $\mathrm{FeCl}_{2}$ facilitated an increase in overall bacterial numbers.

Data on the effects of soluble $\mathrm{Fe}$, however, are less clear. $\mathrm{Fe}$ as a sulfate $\left(\mathrm{FeSO}_{4}\right)$ inhaled at doses of 101-170 $\mu \mathrm{g} / \mathrm{m}^{3}$ did not alter clearance of Staphylococcus aureus, or enhance infection with Group C streptococci (Goldstein et al., 1979). However, inhalation of $200-400 \mu \mathrm{g} / \mathrm{m}^{3}$ did significantly decrease the ability of mice to inactivate Group C streptococci (Sherwood et al., 1981). In the latter study, 200-400 $\mu \mathrm{g} / \mathrm{m}^{3}$ of $\mathrm{FeSO}_{4}$ did not alter clearance of S. aureus. Hatch et al. (1981) showed that mice intratracheally exposed to ferric ammonium sulfate $\left(\mathrm{FeNH}_{4}\left(\mathrm{SO}_{4}\right)_{2}\right)$ at a dose of approximately $4 \mu \mathrm{g} /$ mouse, but not ferric sulfate $\left(\mathrm{Fe}_{2}\left(\mathrm{SO}_{4}\right)_{3}\right)$, underwent a moderate 
increase in mortality after S. pyogenes infection. Whereas, Ehrlich (1980) found no alterations in mortality in mice exposed via inhalation to $\mathrm{FeNH}_{4}\left(\mathrm{SO}_{4}\right)_{2}$ or $\mathrm{Fe}_{2}\left(\mathrm{SO}_{4}\right)_{3}$, at a dose of approximately $2 \mathrm{mg} / \mathrm{m}^{3}$, prior to infection with S. pyogenes or Klebsiella pneumoniae. In the current study, the I.T. dose of $\mathrm{FeSO}_{4}(37.2 \mu \mathrm{g} / \mathrm{rat})$ did not enhance infectivity, and $\mathrm{Fe}$ in the +3 valence state was not examined.

Variable results also exist for $\mathrm{Al}$ in the sulfate form $\left(\mathrm{Al}_{2}\left(\mathrm{SO}_{4}\right)_{3}\right)$. Hatch et al. (1981) and Ehrlich (1980) showed that mice exposed either by I.T. or inhalation to $\left(\mathrm{Al}_{2}\left(\mathrm{SO}_{4}\right)_{3}\right)$ did not undergo significant increases in mortality after infection with $S$. pyogenes, although I.T. of $\mathrm{Al}$ ammonium sulfate $\left(\mathrm{AlNH}_{4}\left(\mathrm{SO}_{4}\right)_{2}\right)$ did alter mortality. However, Drummond et al. (1986) found that inhalation of either $\mathrm{Al}_{2}\left(\mathrm{SO}_{4}\right)_{3}$ or $\mathrm{AlNH}_{4}\left(\mathrm{SO}_{4}\right)_{2}$ resulted in a slight, but significant, increase in infectivity in mice infected with Streptococcus zooepidemicus. In the current study, $\mathrm{Al}$ did not alter clearance of $L$. monocytogenes from the lungs of rats, and may even have had antagonistic effects on the increase in susceptibility induced by Ni. Discrepancies in the findings may be due to dose, route of exposure, and quantity and type of bacteria used.

The reduction in bacterial clearance at the early time point post-infection likely indicates that innate immune responders, such as AMs or NK cells, are being inhibited by soluble Ni. The immune system as a target for soluble $\mathrm{Ni}$ after inhalation or parenteral injection has been well documented, resulting in reduction in AM phagocytosis (ATSDR, 2005; Adkins et al., 1979; Haley et al., 1990; NTP, 1996a; 1996b; 1996c), NK cell cytotoxicity (Goutet et al., 2000; Smialowicz 1985; 1987), and lymphoid hyperplasia in the lung associated lymph nodes (NTP, 1996a; 1996b; 1996c). Numerous studies have shown that soluble Ni is capable of suppressing AM function (Aranyi et al., 1979; 
Camner et al., 1984; Castranova et al., 1980b; Goutet et al., 2000; Haley et al., 1990;

Schluter et al., 1995; Sunderman et al., 1989). In fact, Adkins et al. (1979) demonstrated

that decreased phagocytosis by AMs in response to inhalation of soluble Ni may be partly responsible for increased susceptibility to respiratory infection with S. pyogenes. The previous study showed that the soluble component of slowed the clearance of bacteria from the lungs and could suppress AM production of NO. It may be possible that soluble $\mathrm{Ni}$ in the current study may have reduced the ability of AMs to effectively clear the $L$. monocytogenes infection early after infection.

The pro-inflammatory cytokine cascade, which includes TNF- $\alpha$, IL-6, and IL-12, is a critical step in the innate immune response to L. monocytogenes and leads to a progression that results in initiation of cell-mediated immunity and further activation of innate responders (Seder and Gazzinelli, 1999). IL-6 was significantly increased after infection, on day 6 , in rats pre-exposed to soluble $\mathrm{Ni}$ alone or in combination with other metals when compared to all groups that did not contain Ni (Figure 3.1.7B \& 3.2.7B). The elevation in IL-6 is indicative of increased lung injury and an exacerbated acutephase response due to a possible delay in, or the suppression of, the early innate immune response that lead to the increased bacterial burden on day 6 . The neutrophil response to L. monocytogenes infection is essential, and animals that are deficient in IL-6 have a low neutrophil influx into the lungs and do not survive infection (Dalrymple et al., 1995; Kopf et al., 1994). IL-6 has also been shown to be involved in the initiation of cytotoxic T cell (CD8+) responses (Akira et al., 1990; Ford et al., 1991; Lotz et al., 1988; Renauld et al., 1989). In the current, study, on day 6, the high levels of IL-6 observed in rats treated with soluble $\mathrm{Ni}$ or groups that contained $\mathrm{Ni}$, correlated well with increased 
neutrophil infiltration in to the lungs, as well as increased $\mathrm{T}$ cell recruitment later at day 10. Similar results were observed with the soluble fraction of ROFA in study 2 . These results may indicate a heightened response to a more severe infection on day 6 .

The pro-inflammatory cytokines, including TNF- $\alpha$, IL-12, and IL-6, as well as IL-18, are also known to induce production of ROS and NO (antimicrobial agents) by macrophages and neutrophils, either directly or through the induction of IFN- $\gamma$ production (Billiau, 1996; Borish et al., 1989; Neighbors et al., 2001; Swain, 2001). The elevation in IL-6 on day 6 in groups that contain Ni corresponds well to the elevation in NO in the same groups at that time point (Figure 3.1.5 \& 3.2.5). In study 2, there was a similar increase in NO in the animals exposed to the soluble ROFA fraction, and this was more likely attributed to the neutrophil response due to a likely suppression in AM activity. An identical pattern of lung injury and increased IL-6 and NO production exists in animals that received soluble $\mathrm{Ni}$, alone or in a metal combination, as compared to that observed with the soluble fraction of ROFA in study 2. Therefore, the soluble Ni in ROFA is likely responsible for the excessive inflammation, increased NO production by neutrophils, and the possible suppression of AM activity observed after infection in the lungs of rats pre-exposed to the soluble metal of ROFA. There were significant increases in ROS production, as measured by zymosan-stimulated CL, on day 6 in the groups that received metal mixtures containing Ni. Although, Ni alone did not alter CL on a per cell basis after infection, due the increased influx of cells into the lungs of Ni-treated rats, there was likely an increased oxidant burden in all rats treated with Ni. The oxidative stress may further compromise the hosts' ability to clear the infection. 
Antigen-presenting cells, including macrophages and DCs, produce IL-12 and IL18 which are highly involved in directing the development of the $\mathrm{T}_{\mathrm{H}} 1$ subset of CD4+ T cells (Hsieh et al., 1993; Stoll et al., 1998; Swain, 2001; Trinchieri, 1995), and directing IFN- $\gamma$ production by NK cells (Chan et al., 1992; Naume et al., 1993; Okamura et al., 1995; Tsutsui et al., 1996) and CD8+ T cells (Berg et al., 2002), resulting in further bactericidal activity by macrophages. IL-18 has also been shown to stimulate macrophages directly to produce TNF- $\alpha$ and NO, which would result in increased bacterial killing (Neighbors et al., 2001). Interestingly, IL-12 was significantly elevated after infection in the rats pre-treated with soluble $\mathrm{Ni}$ alone on day 6 , a response that was not replicated in any of the metal mixtures (Figure 3.1.9A \& 3.2.9A), and was not observed with the soluble fraction of ROFA in study 2. In addition, IL-12 was lower, although not significantly, in the rats that were exposed to the Mix-No Ni solution when compared to most of the other metal mixtures and control. It is possible that there are metals that produce the opposite effects as soluble $\mathrm{Ni}$, resulting in a reduction of the response when in a mixture with soluble Ni. IL-18 was also significantly elevated after infection on days 8 and 10 in the rats pre-treated with soluble Ni alone (Figure 3.1.9B). There was a trend for increased IL-18 on day 8 in the metal mixtures that contained $\mathrm{Ni}$, although the differences for control and Mix- No Ni were not significant (Figure 3.1.9B). The IL-18 response was similar to that observed for the soluble ROFA sample in study 2 . As mentioned earlier, IL-12 and IL-18 are produced by macrophages and DCs. Although macrophage function and bacterial clearance appeared to be inhibited by soluble $\mathrm{Ni}, \mathrm{DC}$ cytokine production and antigen-presentation may be unaffected, or even stimulated. Soluble Ni is a known dermal sensitizer, and the effects of this metal on DCs in the skin, 
Langerhans cells, are better investigated than effects on DCs in the lung (ATSDR, 2005; De Smedt et al., 2001; Manome et al., 1999). De Smedt et al. (2001) showed that DCs exposed to soluble Ni had altered cell surface markers and increased production of IL-6, TNF- $\alpha$, and IL-12. In addition, Manome et al. (1999) demonstrated that $\mathrm{NiCl}_{2}$ could induce maturation of DCs and enhance their ability to stimulate $\mathrm{T}$ cells. The stimulatory effect of soluble Ni on DCs may possibly account for the increase in IL-12 and IL-18 observed after infection in the soluble Ni group, and these elevated cytokines may have lead to the increased $\mathrm{T}$ cell influx on day 10 observed in the group of rats treated with soluble Ni (Figure 3.1.4B-D \& 3.2.4B-D).

The adaptive immune response to $L$. monocytogenes is characterized by cellmediated immunity, with CD8+ cytotoxic $\mathrm{T}$ cells and $\mathrm{CD} 4+\mathrm{T}_{\mathrm{H}} 1 \mathrm{~T}$ cells being the primary responders (Pamer, 2004). After infection, T cell numbers increased over time in all groups, attributable to both CD8+ T cells (Figure 3.1.4C \& 3.2.4C) and CD4+ T cells (Figure 3.1.4D \& 3.2.4D), and the T/B cell ratio (Table 3.1.2 \& 3.2.3) also increased over time, as would be expected. In addition, AMs and NK cells also increased. However, on day 10 the increase in $\mathrm{T}$ cell infiltration into the lungs of rats pre-treated with $\mathrm{Ni}$ alone was significantly greater than all groups. There were also slight, but insignificant, increases in $\mathrm{T}$ cells in groups that were pre-treated with mixtures containing $\mathrm{Ni}$ when compared to other individual metals or the control groups (Figure 3.1.4B-D \& 3.2.4B-D). Despite increased $\mathrm{T}$ cells on day 10, $\mathrm{T}$ cell activity appeared to be inhibited, particularly at the early time points post-infection, as indicated by reduced levels of IL-2 and IL-4 (Figure 3.1.10 \& 3.2.10). This response was similar, although not as persistent, as that observed after infection in rats pre-treated with the soluble fraction of ROFA. 
IL-2 plays an important role throughout the course of the infection, in an autocrine fashion, acting as a growth and proliferative factor promoting clonal expansion of $\mathrm{T}_{\mathrm{H}} 1$ and cytotoxic $\mathrm{T}$ cells (Minami et al., 1993), and in a paracrine fashion, activating NK cells (Henney et al., 1981; Naume et al., 1993) and promoting survival of neutrophils (Djeu et al., 1993) and monocytes (Espinoza-Delgado et al., 1995). Activated $\mathrm{T}_{\mathrm{H}} 1$, cytotoxic T, and NK cells will, in turn, secrete cytokines, such as IFN- $\gamma$, which will activate and enhance intracellular killing of bacteria by macrophages. Whether soluble $\mathrm{Ni}$ may be directly affecting $\mathrm{T}$ cells or not is not clear. There are no differences in $\mathrm{T}$ cell recruitment prior to day 10 , yet cytokine production by $\mathrm{T}$ cells in the lung is lower in groups treated with $\mathrm{Ni}$, particularly on day 10 .

Zelikoff et al. (2002) found that respiratory exposure to $\mathrm{NiCl}_{2}$ in uninfected rats altered the ability of splenic lymphocytes to proliferate, and $\mathrm{T}$ cells appeared to be more sensitive to $\mathrm{NiCl}_{2}$ than B cells. Harkin et al. (2003) demonstrated that T lymphocyte proliferation and $\mathrm{T}_{\mathrm{H}} 1$ and $\mathrm{T}_{\mathrm{H}} 2$ cytokine production was suppressed after intraperitoneal injection of $\mathrm{NiCl}_{2}$. Smialowicz et al. (1984) also showed that $\mathrm{T}$ cells, and NK cells, were more sensitive to soluble Ni than B cells when the Ni was administered by intramuscular injection. In the current study, proliferation of T cells and infiltration into the lungs of rats in response to infection does not appear to be inhibited in animals pre-treated with soluble Ni. However, the adverse effects that soluble Ni may have directly on T cells may alter their activity only after the cells enter the lung and are exposed to the Ni.

One possible explanation for inhibition of $\mathrm{T}$ cell activity may be the significant elevations in NO present in the groups that contained soluble Ni. Elevated levels of NO can result in inhibition of T cell proliferation (Blesson et al., 2002; Hoffman et al., 2002; 
van der Veen et al., 1999; 2000) and reduction in IL-2 production (Blesson et al., 2002). In addition, $\mathrm{NiCl}_{2}$ has also been shown to cause increased lipid peroxidation and DNA damage in lymphocytes after intraperitoneal injection (Chen et al., 2003a; 2003b; Wozniak and Blasiak, 2002), and that some of the damage could be attenuated with antioxidant treatment. In study 2 , the effects of the soluble fraction of ROFA on T cells were more profound, with decreased IL-2 occurring prior to infection, as well as throughout the course of the infection. However, there are similarities in T cell recruitment, increased BAL NO levels, and decreased BAL IL-2 concentrations between groups of rats that received soluble $\mathrm{Ni}$ and the rats that were treated with the soluble ROFA sample.

The data taken together indicate that soluble Ni is likely responsible for increased injury and inflammation, decreased bacterial clearance, AM inhibition, and adverse effects on T cells that are observed after exposure to the soluble metals of ROFA. The clearance of bacteria and the inhibition in T cell activity were not as persistent in the rats treated with soluble Ni prior to infection as was observed with soluble ROFA exposure. The measurements of the soluble metals in ROFA that were presented in study 1 may be an underestimate of the amount actually present in the soluble fraction due to a small loss during the recovery that occurs during the separation process. Therefore, the soluble Ni content is likely higher than that used in this study. This also means that there is some loss of the others soluble metals as well. At higher doses, these metals may make more of a contribution in terms of infectivity. There are also instances where soluble Ni alone exerts an effect that is not replicated in the rats that are treated with the total mixture of metals, such as the increase in IL-12; or the effects are more prominent than that 
observed with the mixture, such as the differences in clearance and body weight. In other instances, the metal mixtures appear to have more of an effect in combination with each other, such as the increased zymosan-induced ROS production observed on day 6. It is likely the presence of specific metals, as well as the concentrations and interactions of the metals (both agonistic and antagonistic), play an important role in whether a particular air pollutant will induce susceptibility to infection. 


\section{Figures and Tables: Part One}

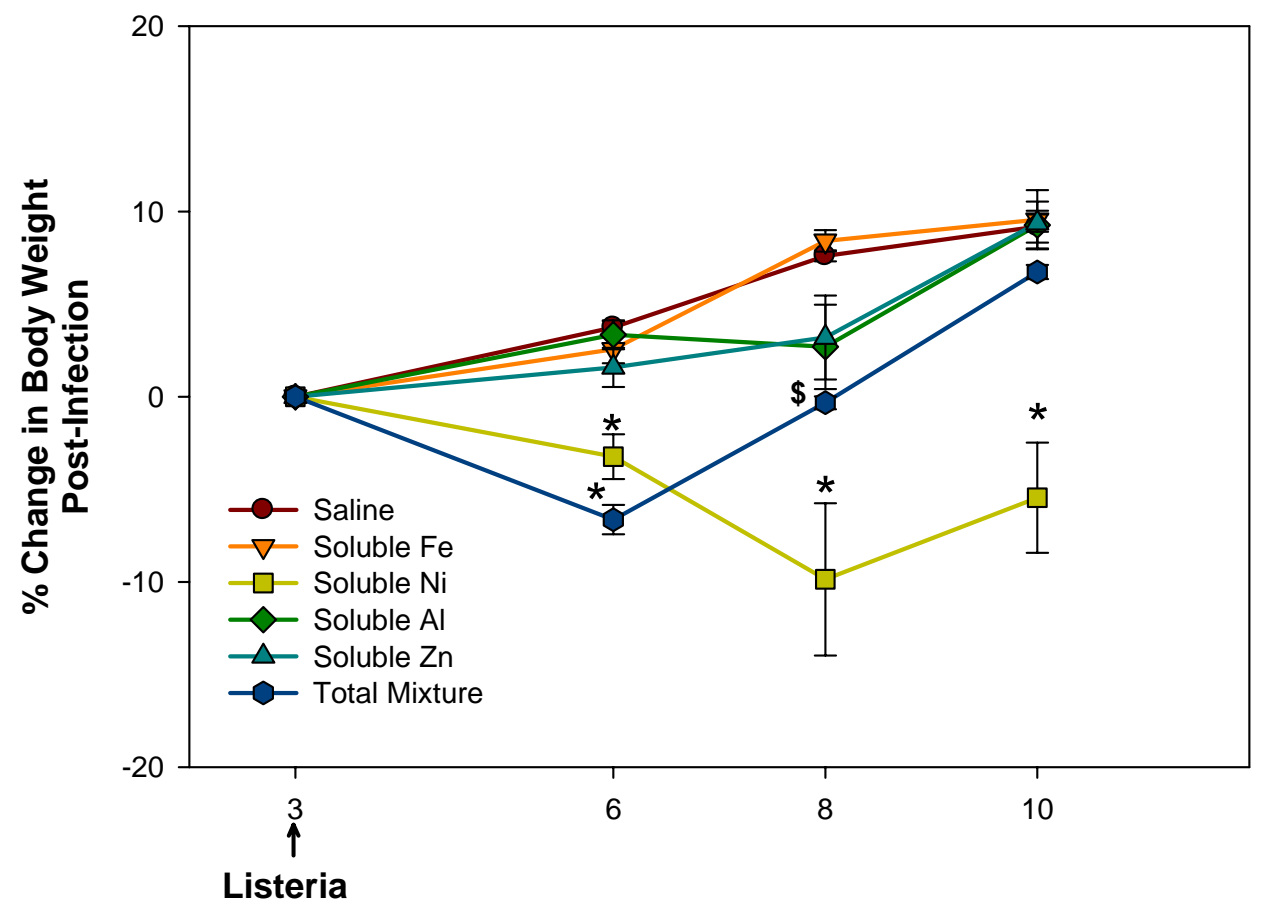

Days (Post-Metal Instillation)

Figure 3.1.1. \% change in body weight post-infection of rats that were pre-exposed to individual soluble metals, the metal mixture, or saline 3 days prior to intratracheal inoculation with $L$. monocytogenes. Values are means $\pm \mathrm{SE}$; * significantly different from all groups; $\$$ significantly different from Soluble Fe and Saline groups $(p<0.05)$. 


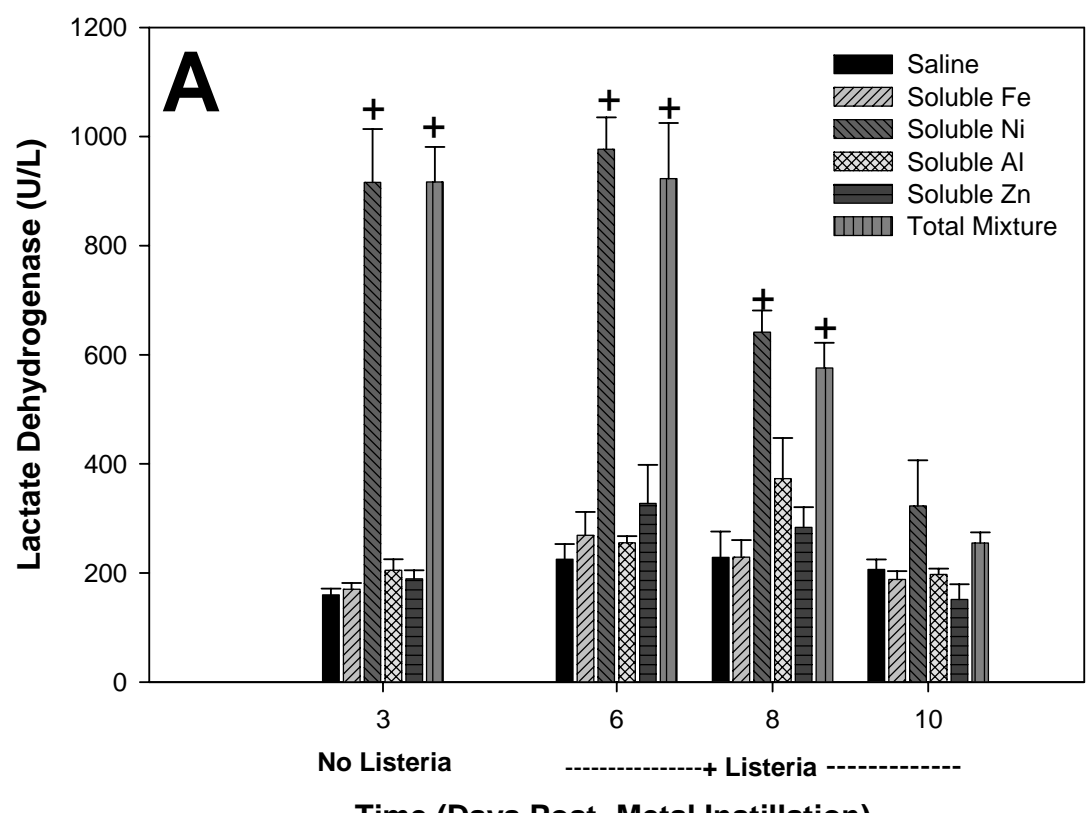

Time (Days Post- Metal Instillation)

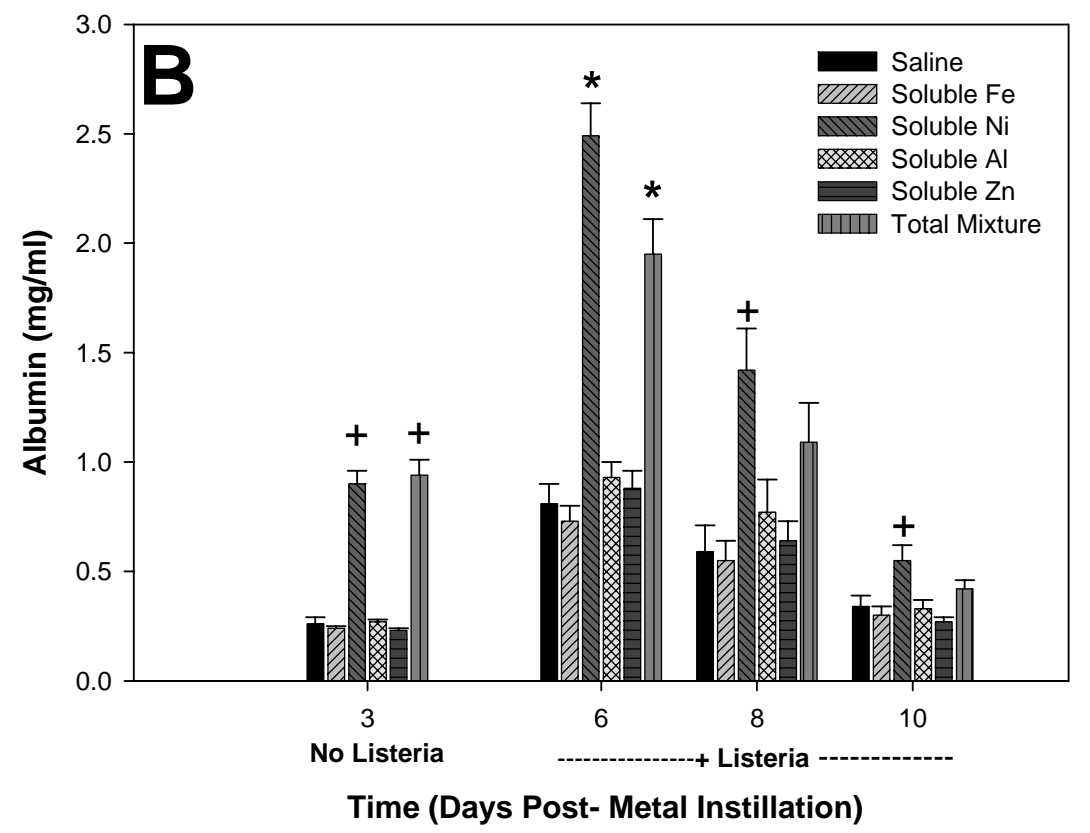

Figure 3.1.2. Lactate dehydrogenase (LDH) (A) and albumin (B) in the BAL fluid of rats that were pre-exposed to individual soluble metals, the metal mixture, or saline 3 days prior to intratracheal inoculation with L. monocytogenes. Values are means $\pm \mathrm{SE}$; * significantly different from all groups; + significantly different from Saline, Soluble Fe, Soluble Al, and Soluble $\mathrm{Zn}$ groups ( $<<0.05)$. 

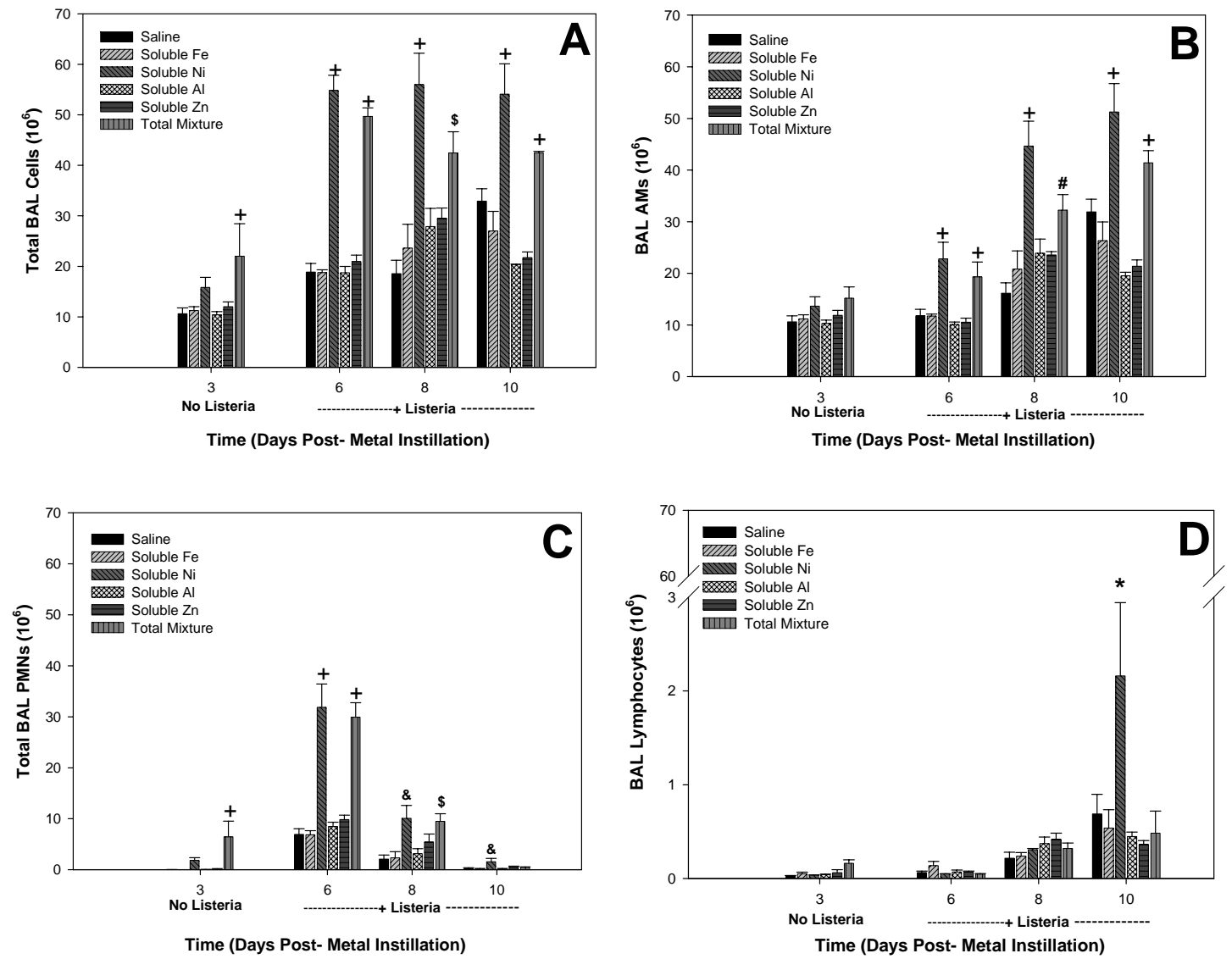

Figure 3.1.3. Total cells (A), totals AMs (B), total PMNs (C), and total lymphocytes (D) present in the BAL of rats that were pre-exposed to individual soluble metals, the metal mixture, or saline 3 days prior to intratracheal inoculation with L. monocytogenes. Values are means $\pm \mathrm{SE}$; * significantly different from all groups; + significantly different from Saline, Soluble Fe, Soluble Al, and Soluble Zn groups; \# significantly different from Saline group; \$ significantly different from Soluble Fe and Saline groups; \& significantly different from Saline, Soluble Fe, and Soluble Al groups ( $\mathrm{p}<0.05)$. 

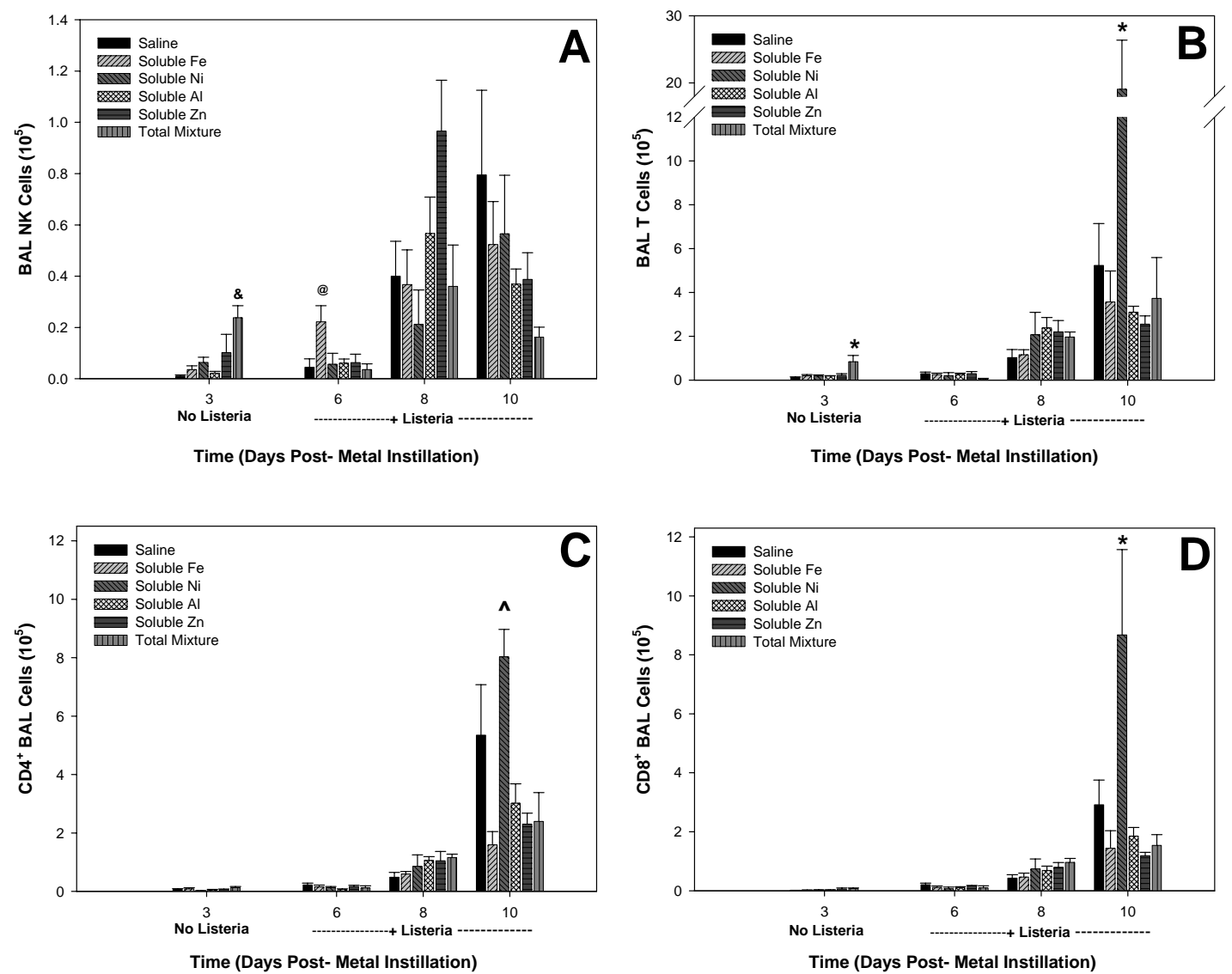

Figure 3.1.4. Total NK cells (A), totals T cells (B), total CD4+ T cells (C), and total $\mathrm{CD} 8+\mathrm{T}$ cells (D) present in the BAL of rats that were pre-exposed to individual soluble metals, the metal mixture, or saline 3 days prior to intratracheal inoculation with $L$. monocytogenes. Values are means \pm SE, * significantly different from all groups; @ significantly different from Saline and Total Mixture groups; \& significantly different from Saline, Soluble Fe, and Soluble Al groups; ^ significantly different from Soluble Fe, Soluble Al, Soluble Zn, and Total Mixture $(\mathrm{p}<0.05)$. 


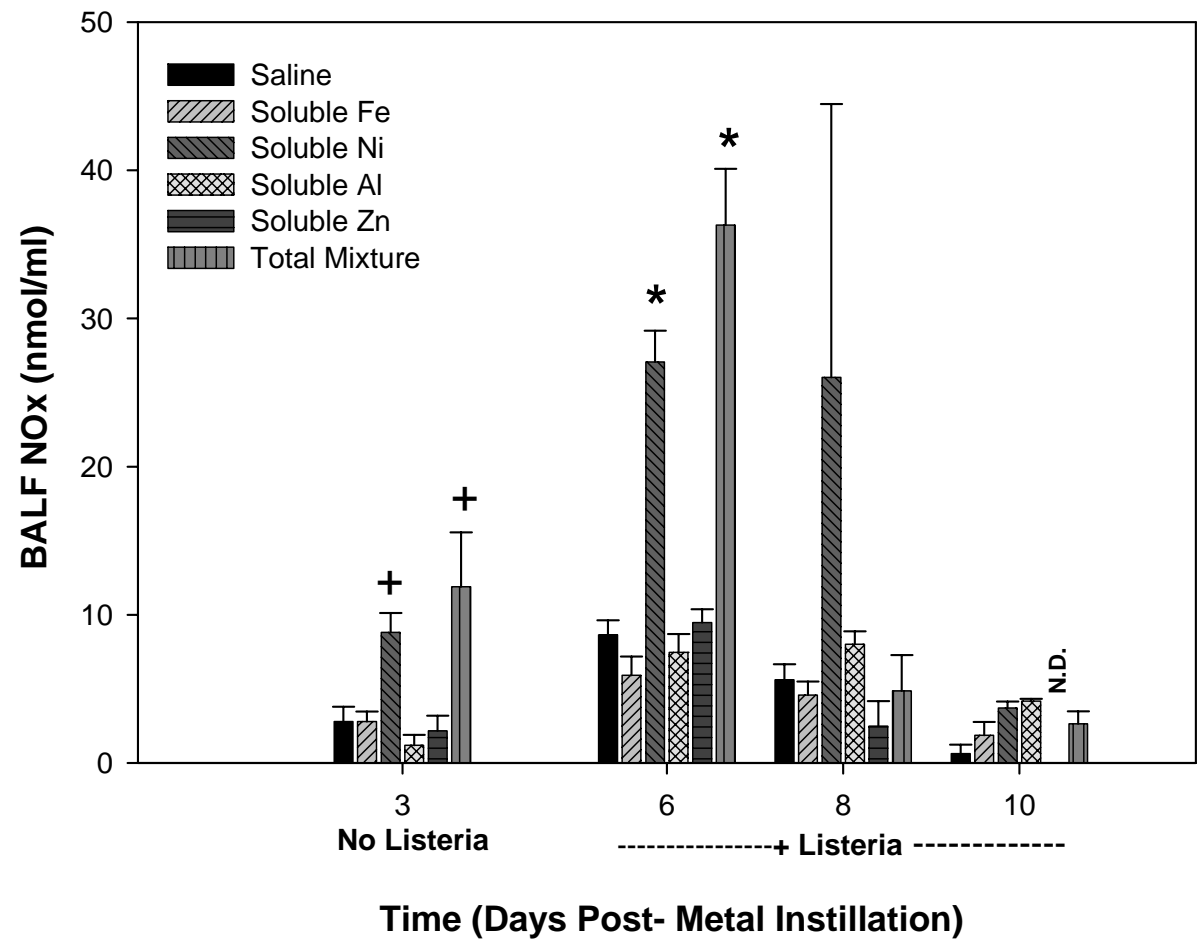

Figure 3.1.5. Nitrate and nitrite (NOx) in the BAL fluid (BALF) of rats that were preexposed to individual soluble metals, the metal mixture, or saline 3 days prior to intratracheal inoculation with L. monocytogenes. Values are means \pm SE; N.D. not determined; * significantly different from all groups; + significantly different from Saline, Soluble Fe, Soluble Al, and Soluble Zn groups ( $\mathrm{p}<0.05)$. 


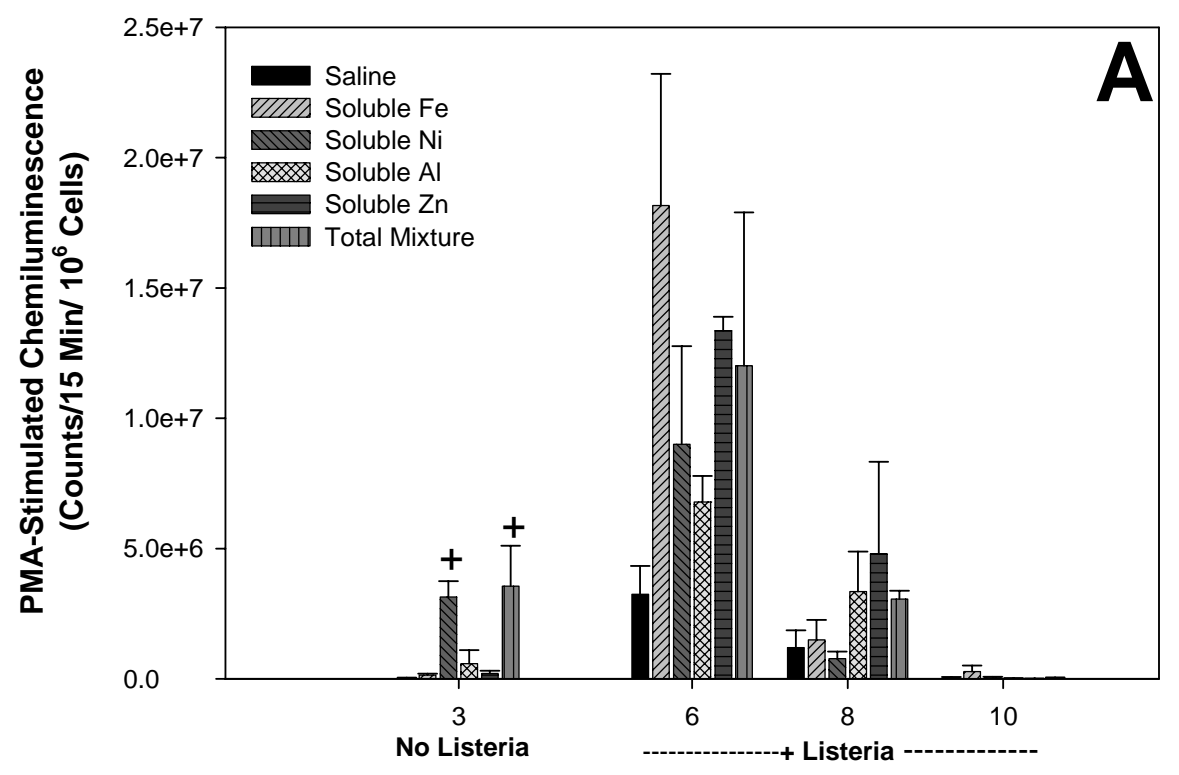

Time (Days Post-Metal Instillation)

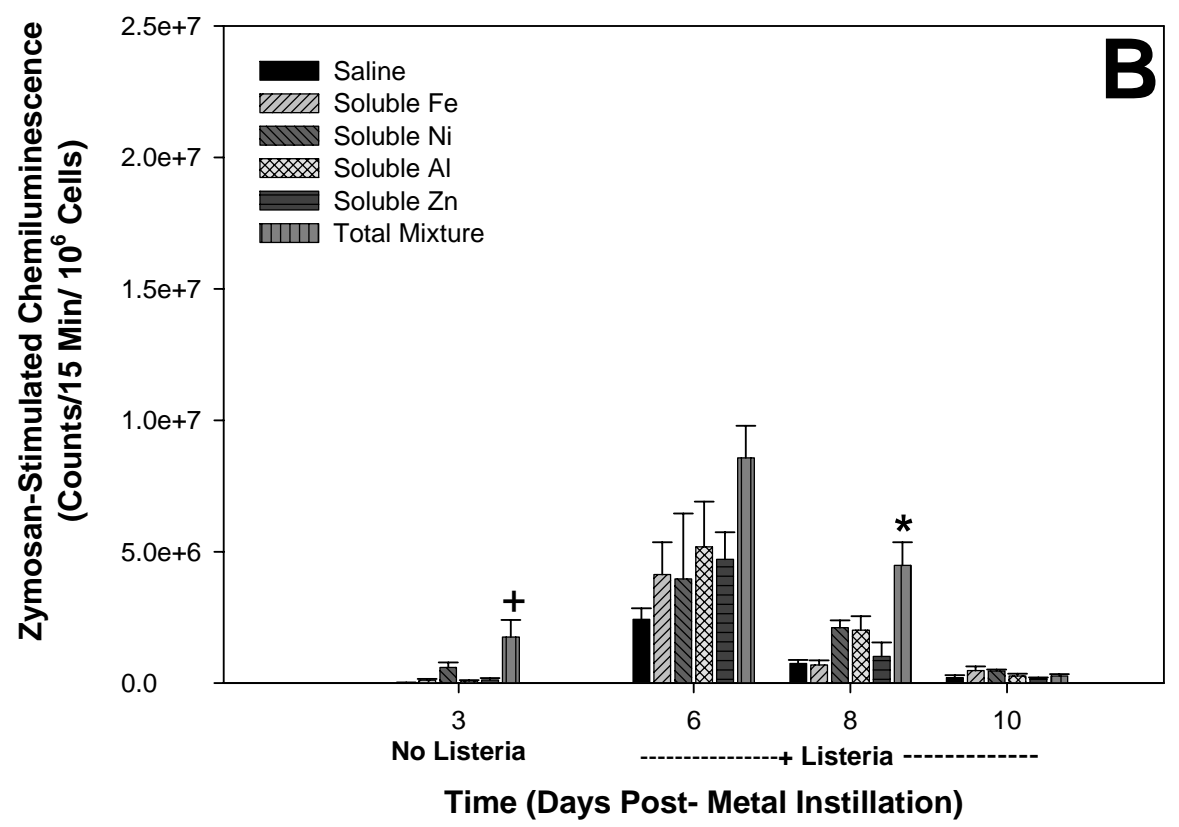

Figure 3.1.6. BAL cellular chemiluminescence (CL) depicted in total counts per $15 \mathrm{~min}$ per $1 \times 10^{6}$ cells after stimulation with PMA (A) or zymosan (B) in rats that were preexposed to individual soluble metals, the metal mixture, or saline 3 days prior to intratracheal inoculation with L. monocytogenes. Values are means $\pm \mathrm{SE}$; * significantly different from all groups; + significantly different from Saline, Soluble Fe, Soluble Al, and Soluble Zn groups ( $\mathrm{p}<0.05)$. 

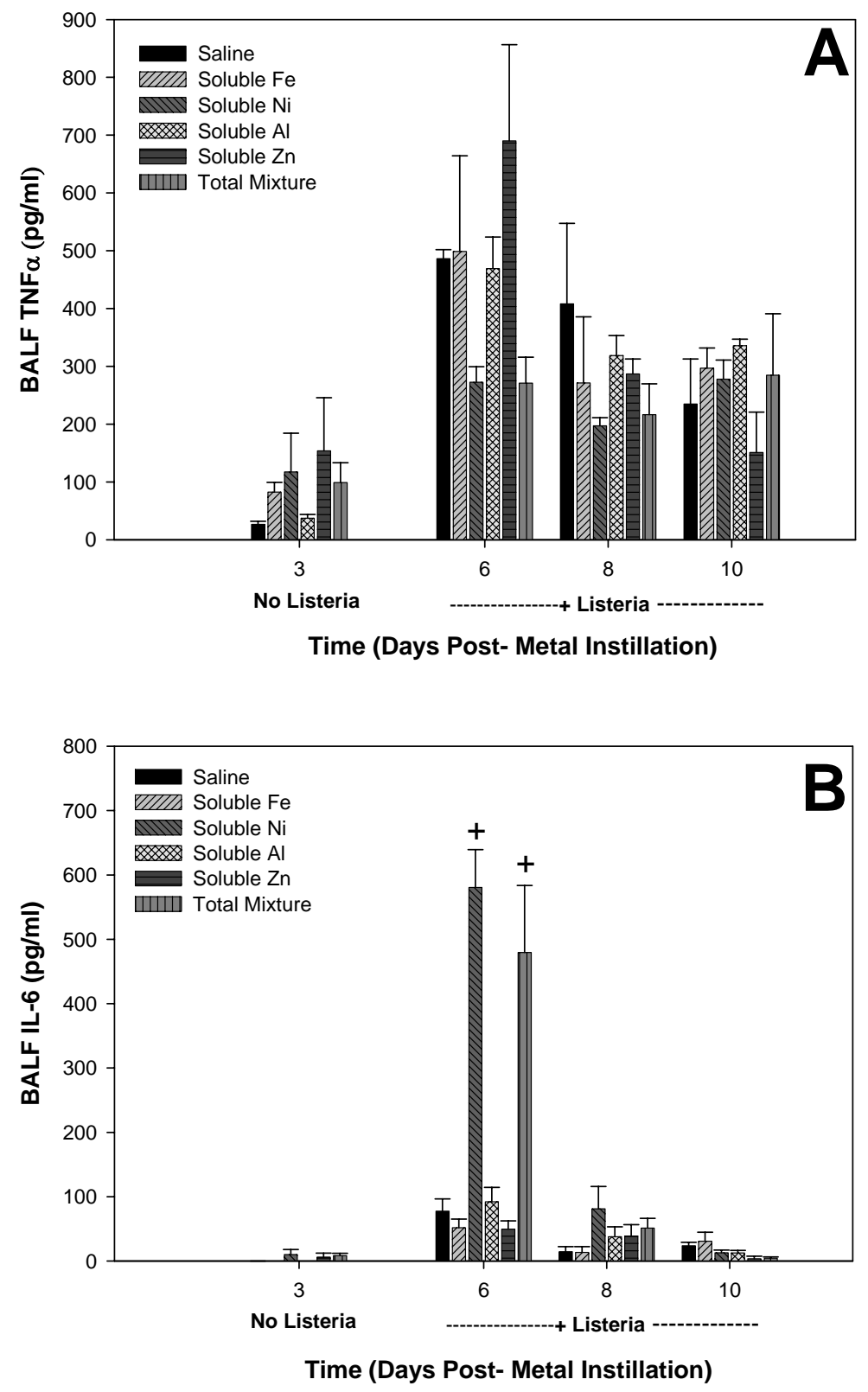

Figure 3.1.7. Concentration of pro-inflammatory cytokine, TNF- $\alpha$ (A), and acute phase response cytokine, IL-6 (B), in the BAL fluid of rats that were pre-exposed to individual soluble metals, the metal mixture, or saline 3 days prior to intratracheal inoculation with L. monocytogenes. Values are means $\pm \mathrm{SE} ;+$ significantly different from Saline, Soluble $\mathrm{Fe}$, Soluble Al, and Soluble $\mathrm{Zn}$ groups $(\mathrm{p}<0.05)$. 


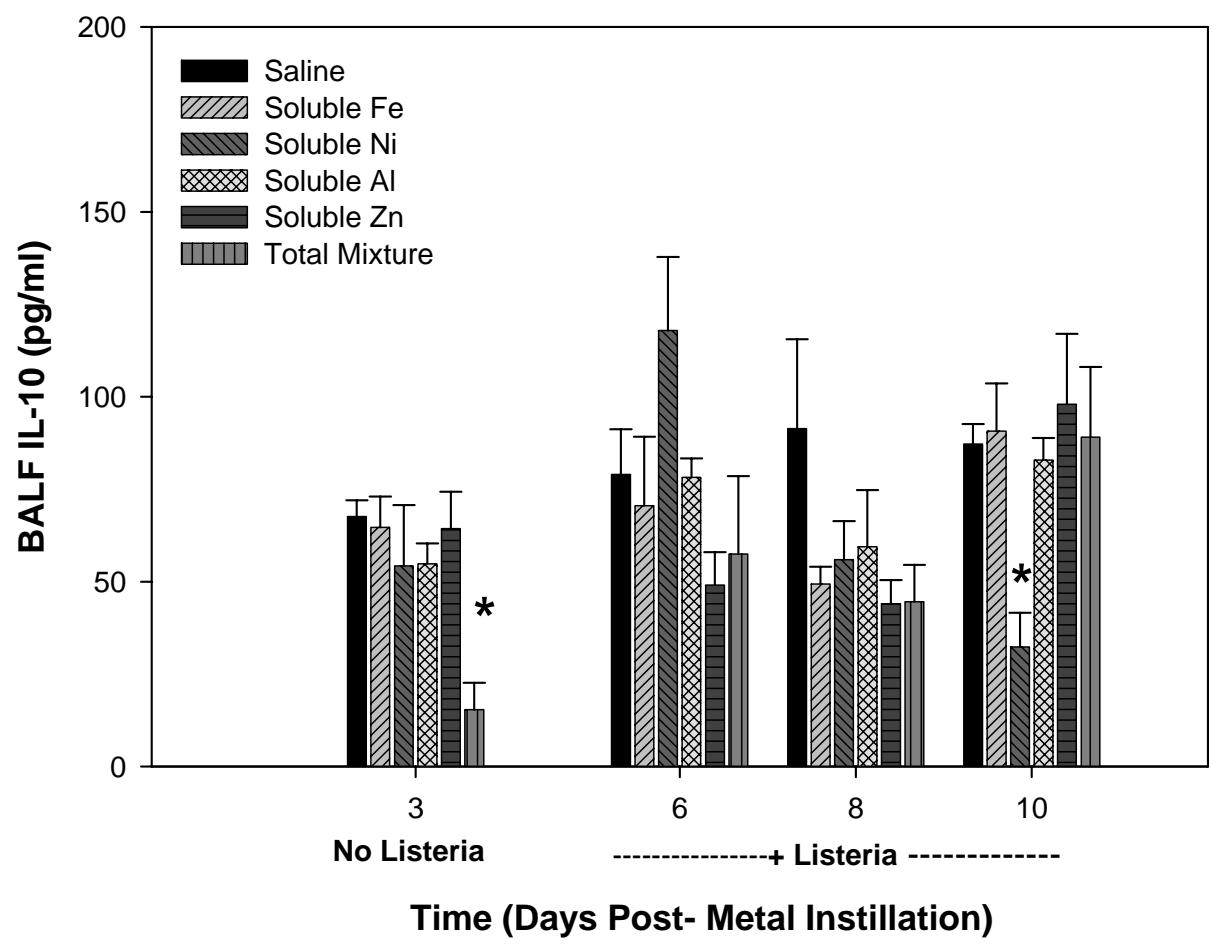

Figure 3.1.8. IL-10, an anti-inflammatory cytokine, in the BAL fluid of rats that were pre-exposed to individual soluble metals, the metal mixture, or saline 3 days prior to intratracheal inoculation with $L$. monocytogenes. Values are means $\pm \mathrm{SE}$; $*$ significantly different from all groups $(\mathrm{p}<0.05)$. 


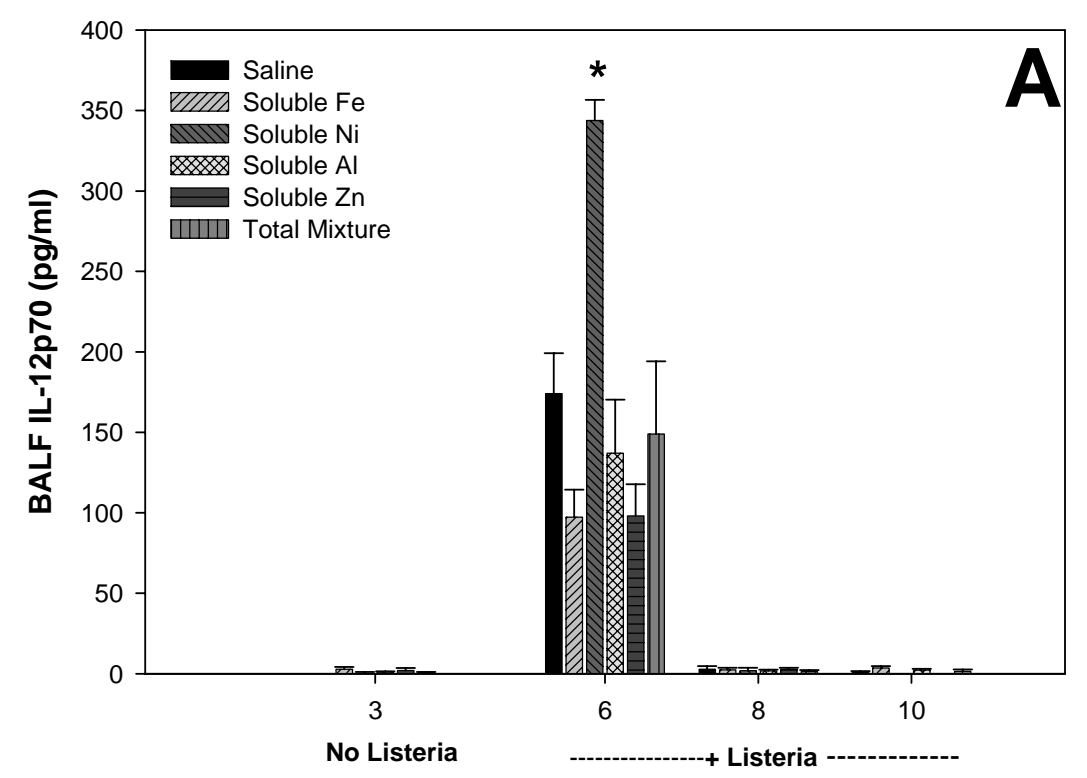

Time (Days Post- Metal Instillation)

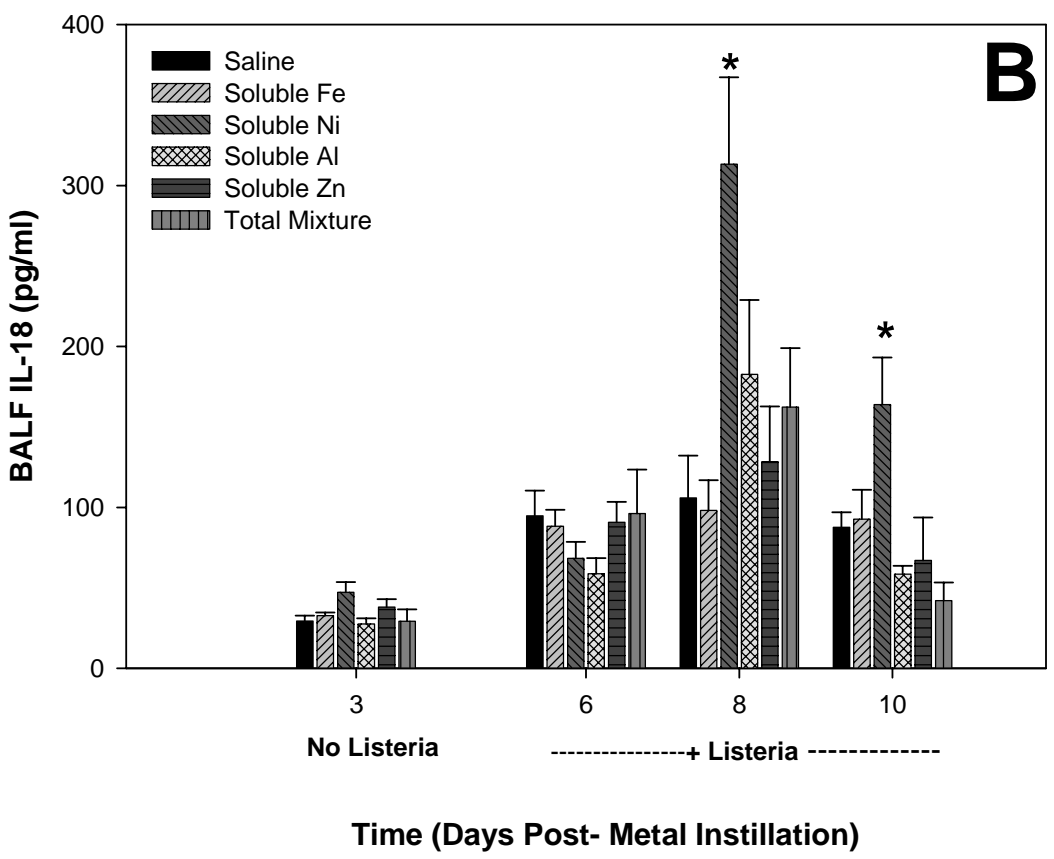

Figure 3.1.9. Cytokines involved in directing the appropriate $\mathrm{T}$ cell response to $L$. monocytogenes, IL-12p70 (A) and IL-18 (B), in the BAL fluid of rats that were preexposed to individual soluble metals, the metal mixture, or saline 3 days prior to intratracheal inoculation with $L$. monocytogenes. Values are means $\pm \mathrm{SE}$; * significantly different from all groups $(\mathrm{p}<0.05)$. 

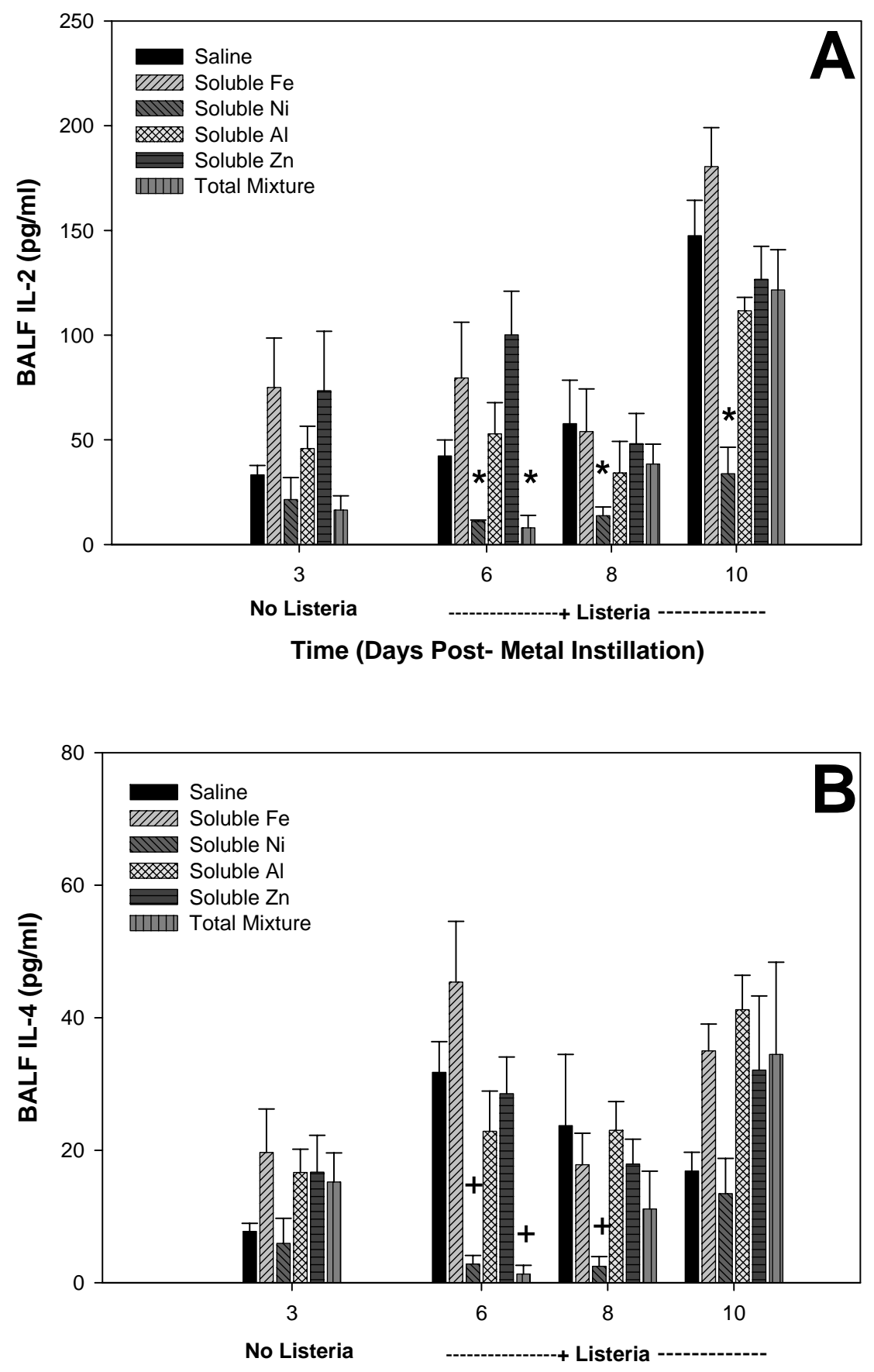

Time (Days Post- Metal Instillation)

Figure 3.1.10. T cell cytokines, IL-2 (A) and IL-4 (B), in the BAL fluid of rats that were pre-exposed to individual soluble metals, the metal mixture, or saline 3 days prior to intratracheal inoculation with $L$. monocytogenes. Values are means $\pm \mathrm{SE}$; * significantly different from all groups; + significantly different from Saline, Soluble Fe, Soluble Al, and Soluble $\mathrm{Zn}$ groups $(\mathrm{p}<0.05)$. 
Table 3.1.1. Bacterial Clearance

\begin{tabular}{ccccc} 
& \multicolumn{4}{c}{ CFUs LOG } \\
\cline { 2 - 5 } Treatment & $\begin{array}{c}\text { Day 3 } \\
\text { Initial Infection }\end{array}$ & Day 6 & Day 8 & Day 10 \\
\hline Saline & 4.7 & $5.9 \pm 0.10$ & $4.3 \pm 0.45$ & $2.2 \pm 0.16$ \\
\hline Soluble Fe & 4.7 & $5.6 \pm 0.03$ & $4.1 \pm 0.02$ & $3.1 \pm 0.04$ \\
\hline Soluble Ni & 4.7 & $7.3 \pm 0.05^{+}$ & $5.7 \pm 0.63$ & $3.2 \pm 0.30$ \\
\hline Soluble Al & 4.7 & $5.9 \pm 0.08$ & $4.3 \pm 0.45$ & $2.2 \pm 1.0$ \\
\hline Soluble Zn & 4.7 & $6.1 \pm 0.14$ & $4.4 \pm 0.45$ & $2.4 \pm 1.0$ \\
\hline Total Mixture & 4.7 & $7.4 \pm 0.45^{+}$ & $4.4 \pm 0.16$ & $2.2 \pm 1.1$ \\
\hline
\end{tabular}

Table 3.1.1. Number of bacterial CFUs in the left lungs of rats that were pre-exposed to individual soluble metals, the metal mixture, or saline 3 days prior to intratracheal inoculation with $L$. monocytogenes. Values are means $\pm \mathrm{SE},+$ significantly different from Saline, Soluble Fe, Soluble Al, and Soluble Zn groups $(\mathrm{p}<0.05)$. 


\section{Table 3.1.2. T/B Cell Ratio}

BAL T/B Cell Ratio

\begin{tabular}{ccccc}
\cline { 2 - 5 } Treatment & $\begin{array}{c}\text { Day 3 } \\
\text { Pre-Infection }\end{array}$ & Day 6 & Day 8 & Day 10 \\
\hline Saline & $0.997 \pm 0.278$ & $1.03 \pm 0.194$ & $1.30 \pm 0.215$ & $5.58 \pm 0.845$ \\
\hline Soluble Fe & $1.49 \pm 0.947$ & $0.396 \pm 0.143$ & $1.48 \pm 0.323$ & $2.58 \pm 0.364$ \\
\hline Soluble Ni & $1.61 \pm 0.185$ & $1.15 \pm 0.759$ & $2.89 \pm 0.277$ & $9.27 \pm 2.38$ \\
\hline Soluble Al & $1.24 \pm 0.479$ & $0.850 \pm 0.296$ & $3.14 \pm 0.445$ & $4.53 \pm 1.49$ \\
\hline Soluble Zn & $1.08 \pm 0.159$ & $1.03 \pm 0.370$ & $2.24 \pm 0.539$ & $3.66 \pm 0.334$ \\
\hline Total Mixture & $1.47 \pm 0.337$ & $0.132 \pm 0.059$ & $3.39 \pm 1.17$ & $4.71 \pm 0.616$
\end{tabular}

Table 3.1.2. Ratio of $\mathrm{T}$ cells to B cells retrieved from the BAL of rats that were preexposed to individual soluble metals, the metal mixture, or saline 3 days prior to intratracheal inoculation with L. monocytogenes. 
Table 3.1.3. CD4+/CD8+ T Cell Ratio

\begin{tabular}{ccccc} 
& \multicolumn{4}{c}{ BAL CD4+/CD8+ T Cell Ratio } \\
\cline { 2 - 5 } Treatment & $\begin{array}{c}\text { Day 3 } \\
\text { Pre-Infection }\end{array}$ & Day 6 & Day 8 & Day 10 \\
\hline Saline & $4.88 \pm 1.64$ & $1.15 \pm 0.347$ & $1.12 \pm 0.177$ & $1.80 \pm 0.097$ \\
\hline Soluble Fe & $3.42 \pm 0.741$ & $1.49 \pm 0.219$ & $1.77 \pm 0.662$ & $1.26 \pm 0.255$ \\
\hline Soluble Ni & $0.958 \pm 0.375$ & $1.50 \pm 0.289$ & $1.32 \pm 0.249$ & $1.19 \pm 0.267$ \\
\hline Soluble Al & $2.38 \pm 1.21$ & $1.04 \pm 0.579$ & $1.81 \pm 0.516$ & $1.59 \pm 0.112$ \\
\hline Soluble Zn & $1.42 \pm 0.531$ & $0.986 \pm 0.264$ & $1.25 \pm 0.287$ & $1.95 \pm 0.251$ \\
\hline Total Mixture & $2.22 \pm 0.696$ & $0.583 \pm 0.344$ & $1.24 \pm 0.139$ & $1.40 \pm 0.232$
\end{tabular}

Table 3.1.3. Ratio of CD4+ T cells to CD8+ T cells retrieved from the BAL of rats that were pre-exposed to individual soluble metals, the metal mixture, or saline 3 days prior to intratracheal inoculation with $L$. monocytogenes. 


\section{Figures and Tables: Part Two}

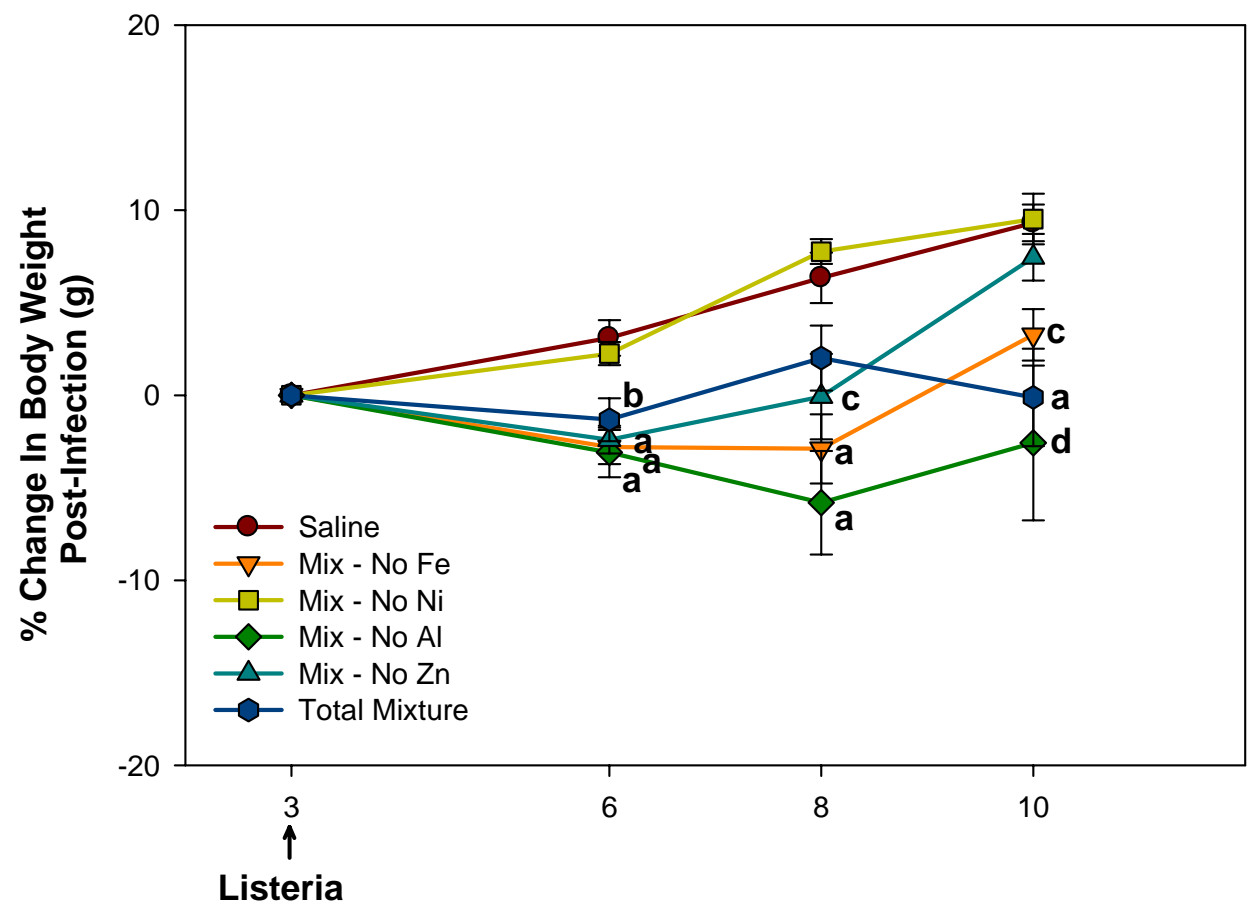

Time (Days Post-Metal Instillation)

Figure 3.2.1. \% change in body weight post-infection of rats that were pre-exposed to soluble metal mixtures, the total metal mixture, or saline 3 days prior to intratracheal inoculation with $L$. monocytogenes. Values are means $\pm \mathrm{SE} ;{ }^{\mathbf{a}}$ significantly different from Saline and Mix - No Ni groups; ${ }^{\mathbf{b}}$ significantly different from Saline group; ${ }^{\mathbf{c}}$ significantly different from Mix - No Ni group; ${ }^{\mathbf{d}}$ significantly different from Saline, Mix - No Ni, and Mix - No Zn groups $(\mathrm{p}<0.05)$. 

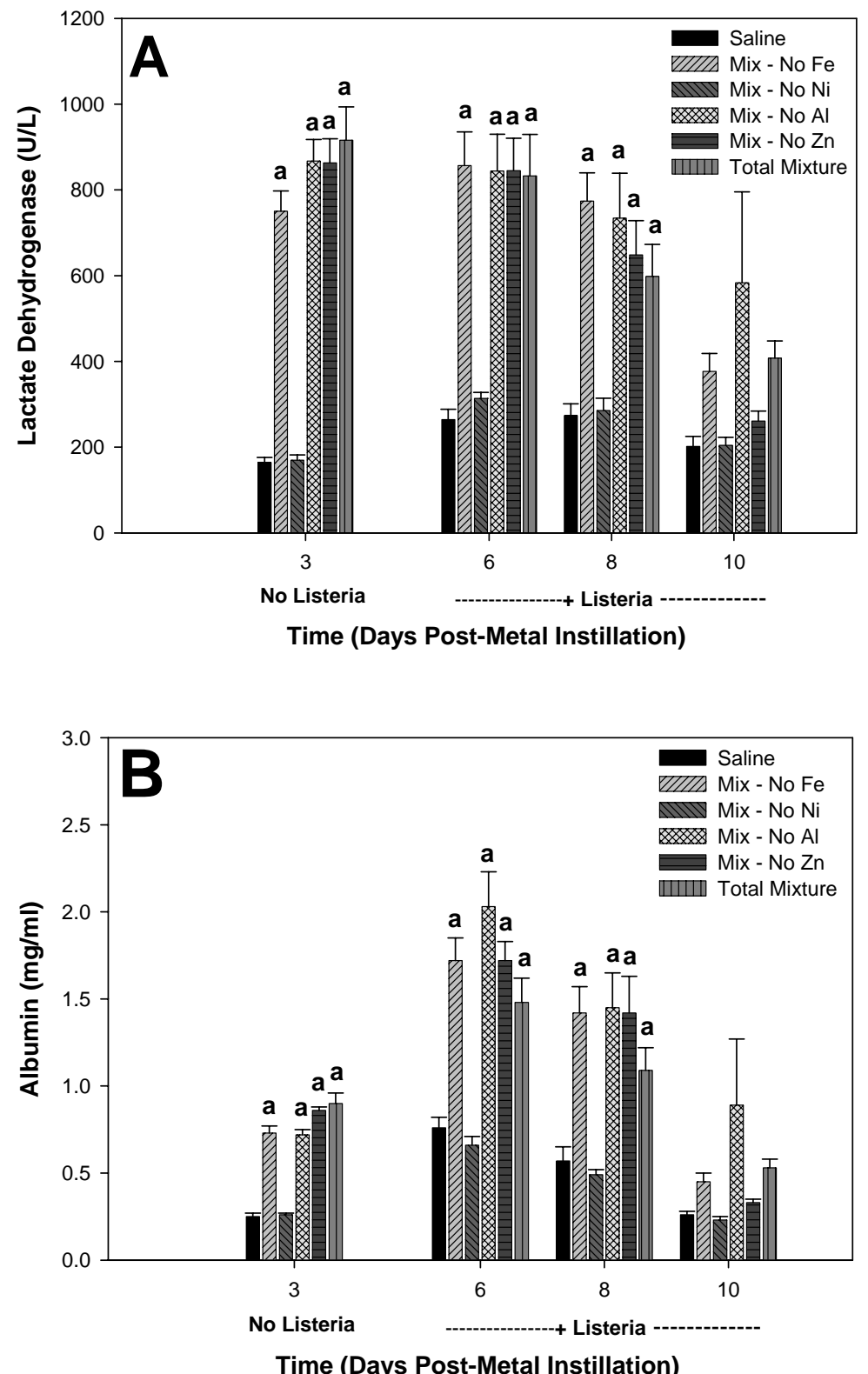

Figure 3.2.2. Lactate dehydrogenase (LDH) (A) and albumin (B) in the BAL fluid of rats that were pre-exposed to soluble metal mixtures, the total metal mixture, or saline 3 days prior to intratracheal inoculation with $L$. monocytogenes. Values are means $\pm \mathrm{SE}$;

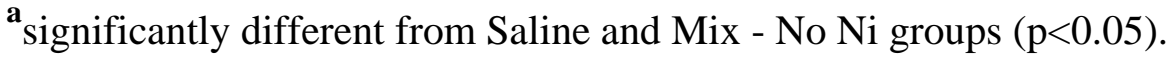



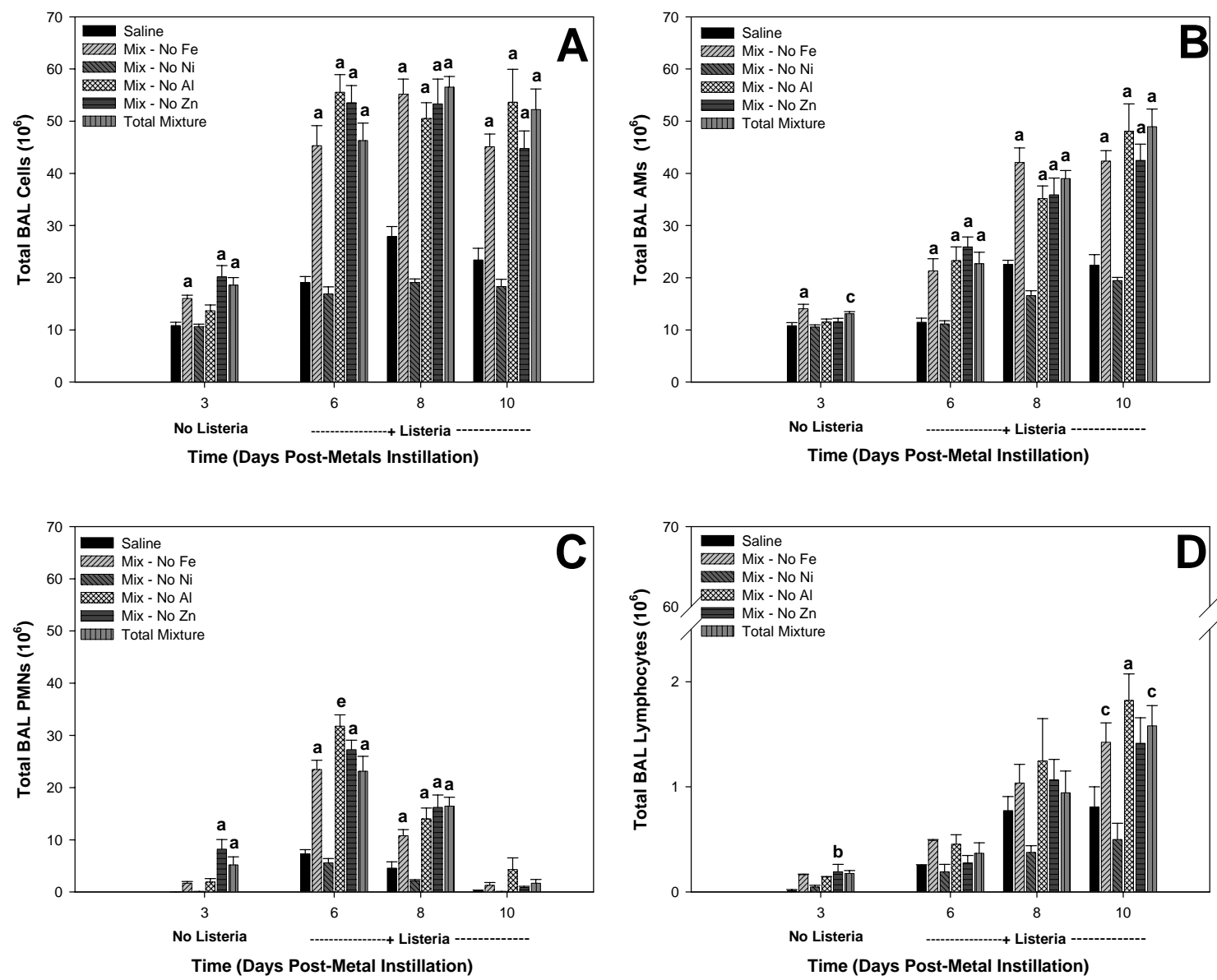

Figure 3.2.3. Total cells (A), totals AMs (B), total PMNs (C), and total lymphocytes (D) present in the BAL of rats that were pre-exposed to soluble metal mixtures, the total metal mixture, or saline 3 days prior to intratracheal inoculation with L. monocytogenes. Values are means $\pm \mathrm{SE} ;{ }^{\mathbf{a}}$ significantly different from Saline and Mix - No Ni groups; $\mathbf{b}_{\text {significantly different from Saline group; }}{ }^{\mathbf{c}}$ significantly different from Mix - No Ni group; ${ }^{\mathbf{e}}$ significantly different from Saline, Mix - No Fe, and Mix - No Ni groups $(\mathrm{p}<0.05)$. 

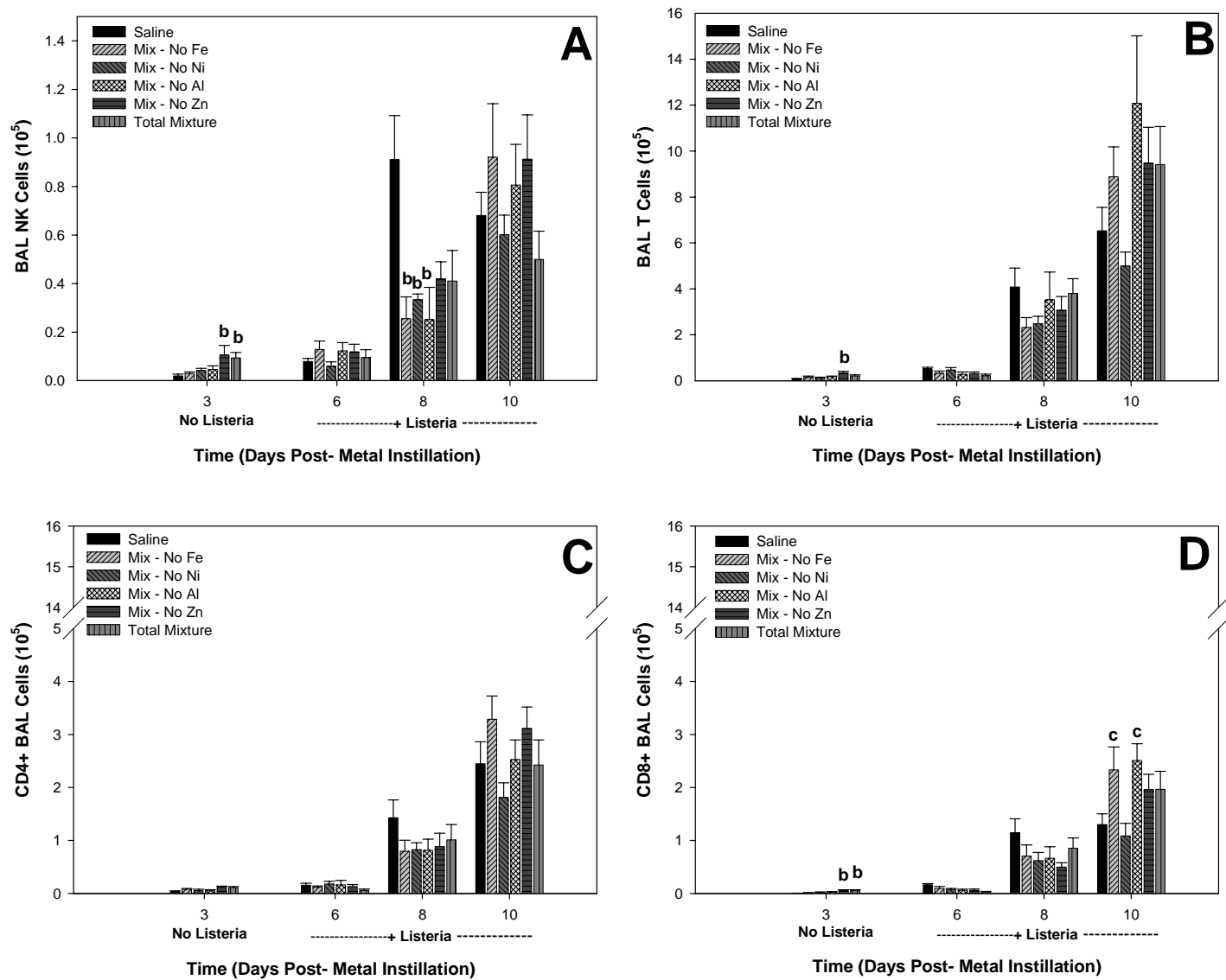

Figure 3.2.4. Total NK cells (A), totals T cells (B), total CD4+ T cells(C), and total CD8+ T cells (D) present in the BAL of rats that were pre-exposed to soluble metal mixtures, the total metal mixture, or saline 3 days prior to intratracheal inoculation with L. monocytogenes. Values are means $\pm \mathrm{SE} ;{ }^{\mathbf{b}}$ significantly different from Saline group; cignificantly different from Mix - No Ni group ( $\mathrm{p}<0.05)$. 


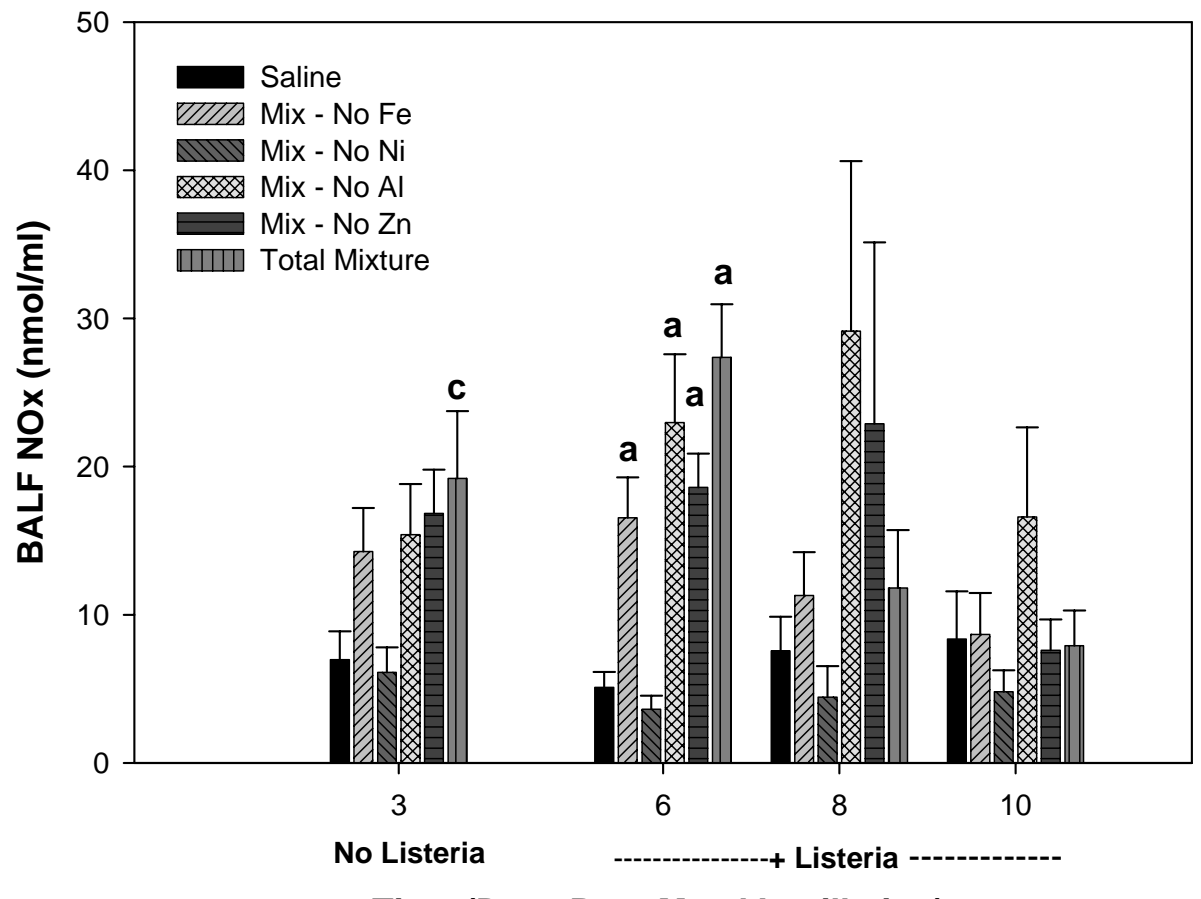

Time (Days Post-Metal Instillation)

Figure 3.2.5. Nitrate and nitrite (NOx) in the BAL fluid (BALF) of rats that were preexposed to soluble metal mixtures, the total metal mixture, or saline 3 days prior to intratracheal inoculation with $L$. monocytogenes. Values are means $\pm \mathrm{SE} ;^{\mathbf{a}}$ significantly different from Saline and Mix - No Ni groups; ${ }^{\mathbf{c}}$ significantly different from Mix - No Ni group $(\mathrm{p}<0.05)$. 

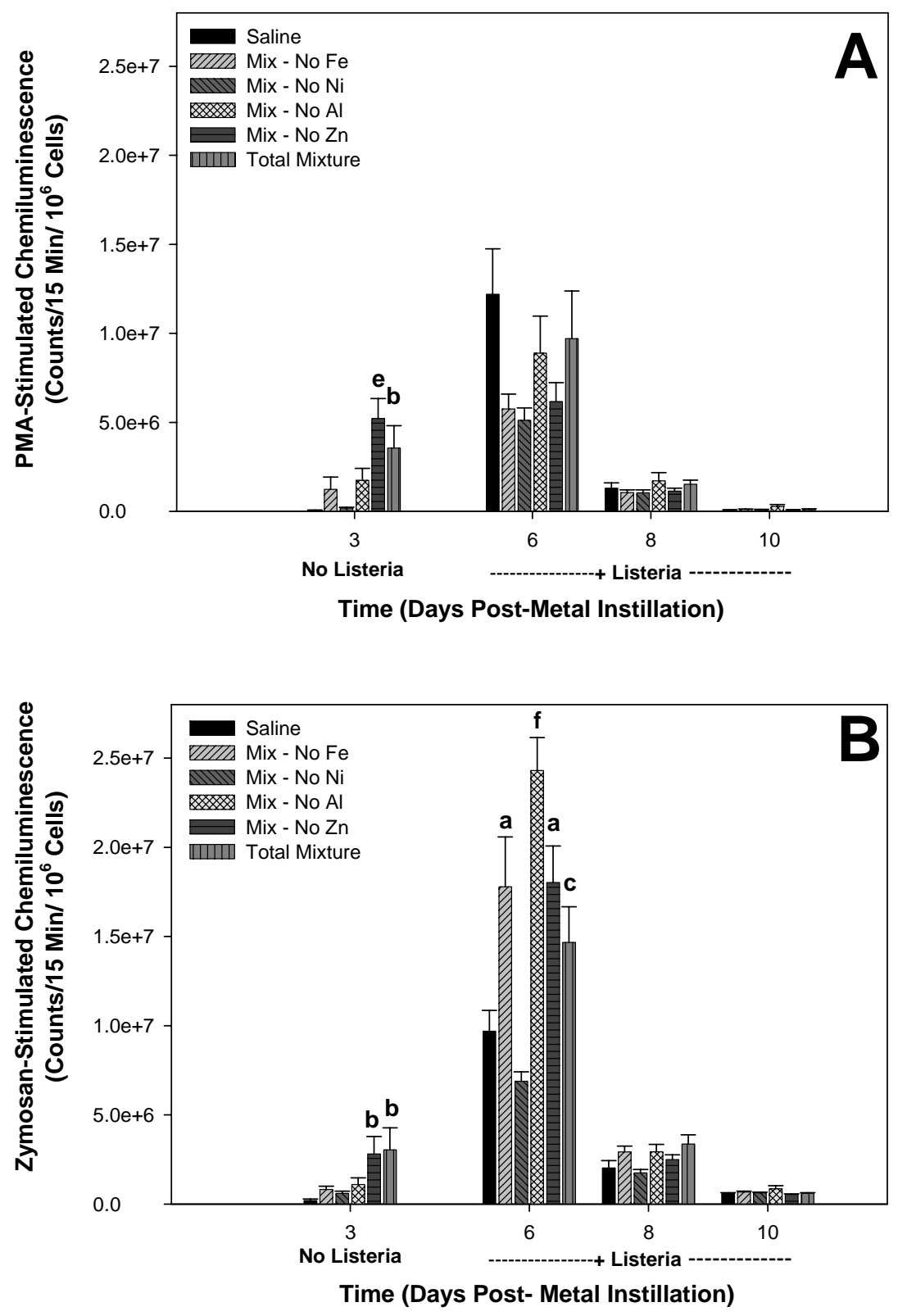

Figure 3.2.6. BAL cellular chemiluminescence (CL) depicted in total counts per 15 min per $1 \times 10^{6}$ cells after stimulation with PMA (A) or zymosan (B) in rats that were preexposed to soluble metal mixtures, the total metal mixture, or saline 3 days prior to intratracheal inoculation with $L$. monocytogenes. Values are means $\pm \mathrm{SE} ;^{\mathbf{a}}$ significantly different from Saline and Mix - No Ni groups; ${ }^{\mathbf{b}}$ significantly different from Saline group; ${ }^{\mathbf{c}}$ significantly different from Mix - No Ni group; ${ }^{\mathbf{e}}$ significantly different from Saline, Mix - No Ni, and Mix - No Fe groups; $\mathbf{f}$ significantly different from all groups $(\mathrm{p}<0.05)$. 

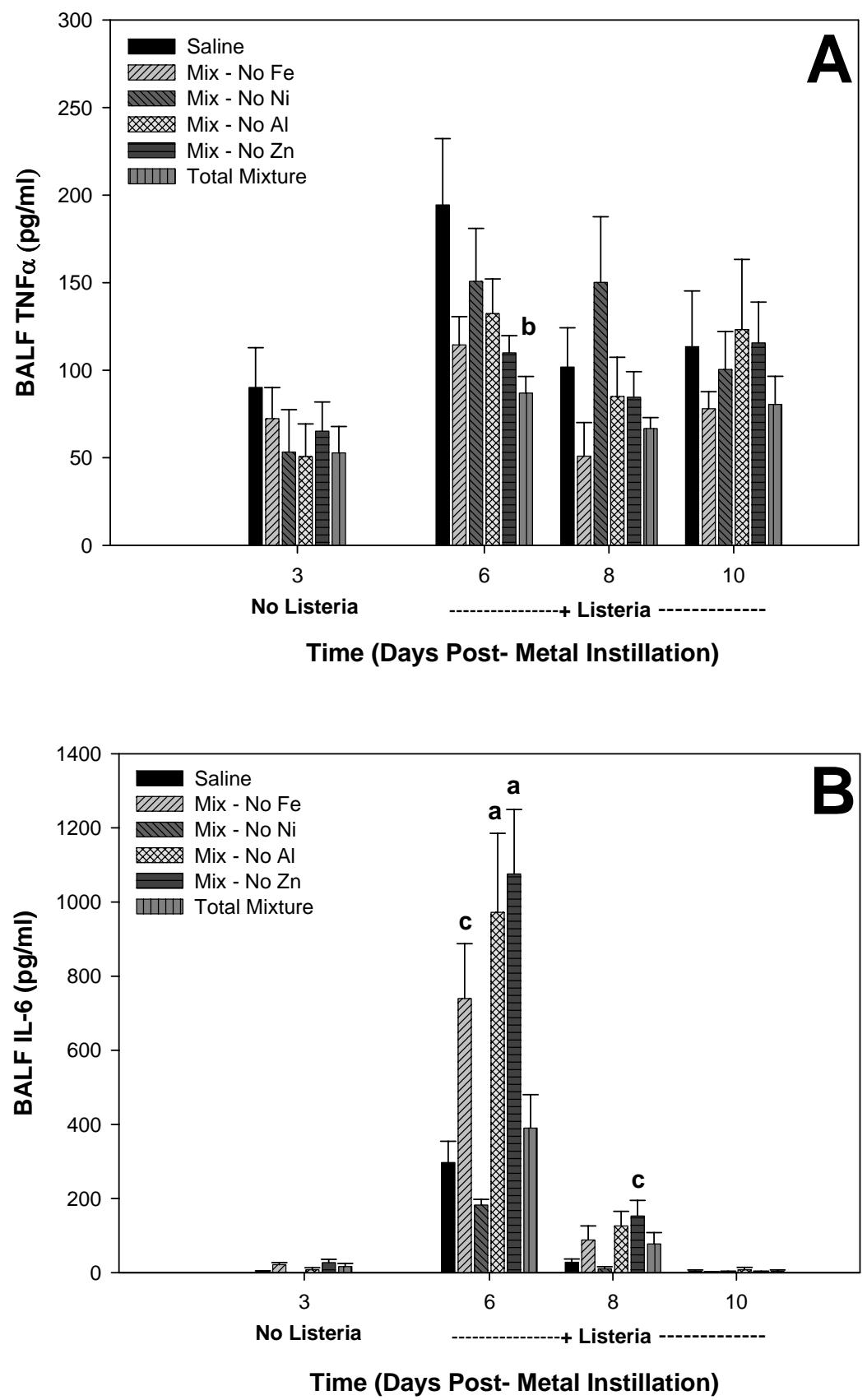

Figure 3.2.7. Concentration of pro-inflammatory cytokine, TNF- $\alpha(\mathrm{A})$, and acute phase response cytokine, IL-6 (B), in the BAL fluid of rats that were pre-exposed to soluble metal mixtures, the total metal mixture, or saline 3 days prior to intratracheal inoculation with $L$. monocytogenes. Values are means $\pm \mathrm{SE} ;{ }^{\mathbf{a}}$ significantly different from Saline and Mix - No Ni groups; ${ }^{\mathbf{b}}$ significantly different from Saline group; ${ }^{\mathbf{c}}$ significantly different from Mix - No Ni group $(\mathrm{p}<0.05)$. 


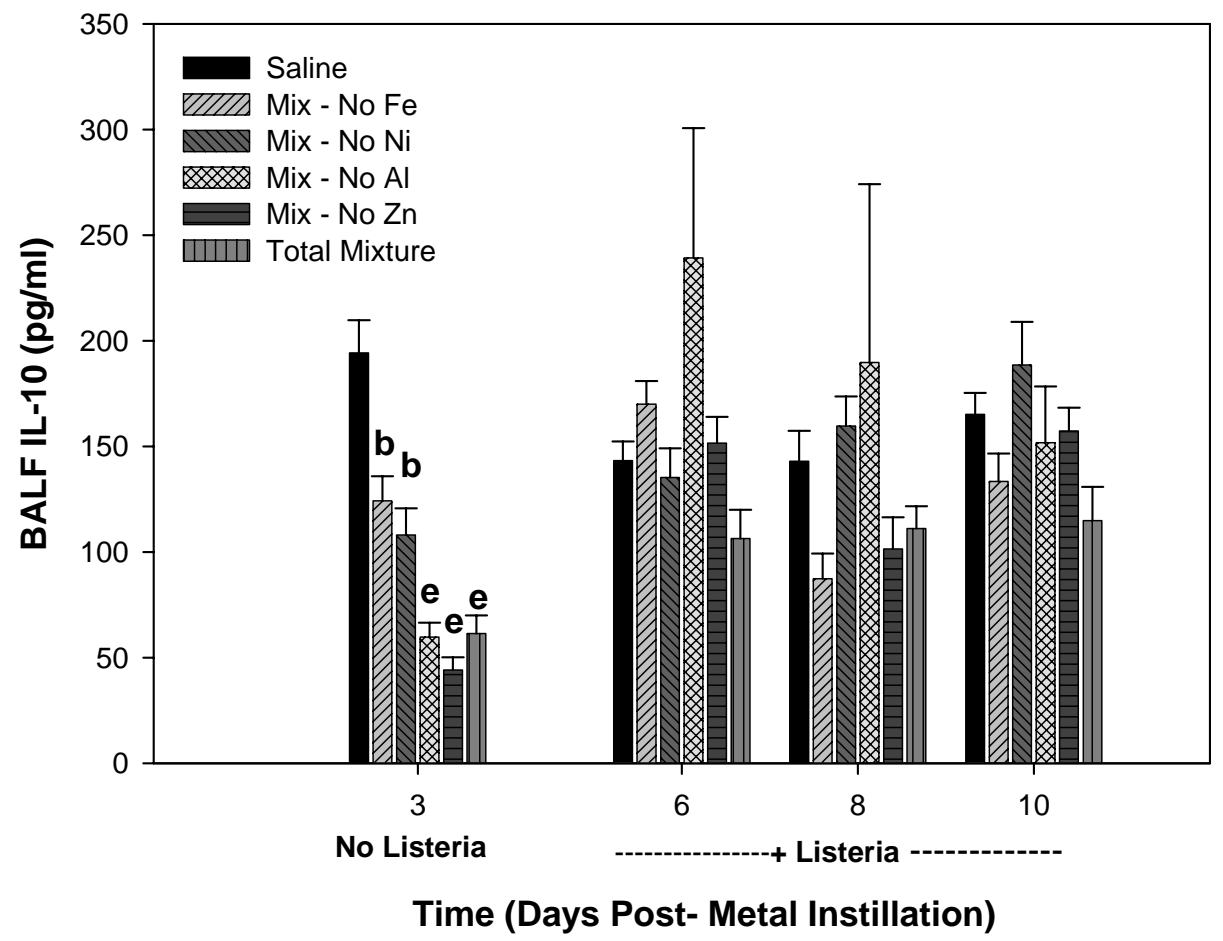

Figure 3.2.8. IL-10, an anti-inflammatory cytokine, in the BAL fluid of rats that were pre-exposed to soluble metal mixtures, the total metal mixture, or saline 3 days prior to intratracheal inoculation with L. monocytogenes. Values are means $\pm \mathrm{SE}$; ${ }^{\mathbf{b}}$ significantly different from Saline group; ${ }^{\mathbf{e}}$ significantly different from Saline, Mix - No Ni, and Mix No Fe groups $(\mathrm{p}<0.05)$. 


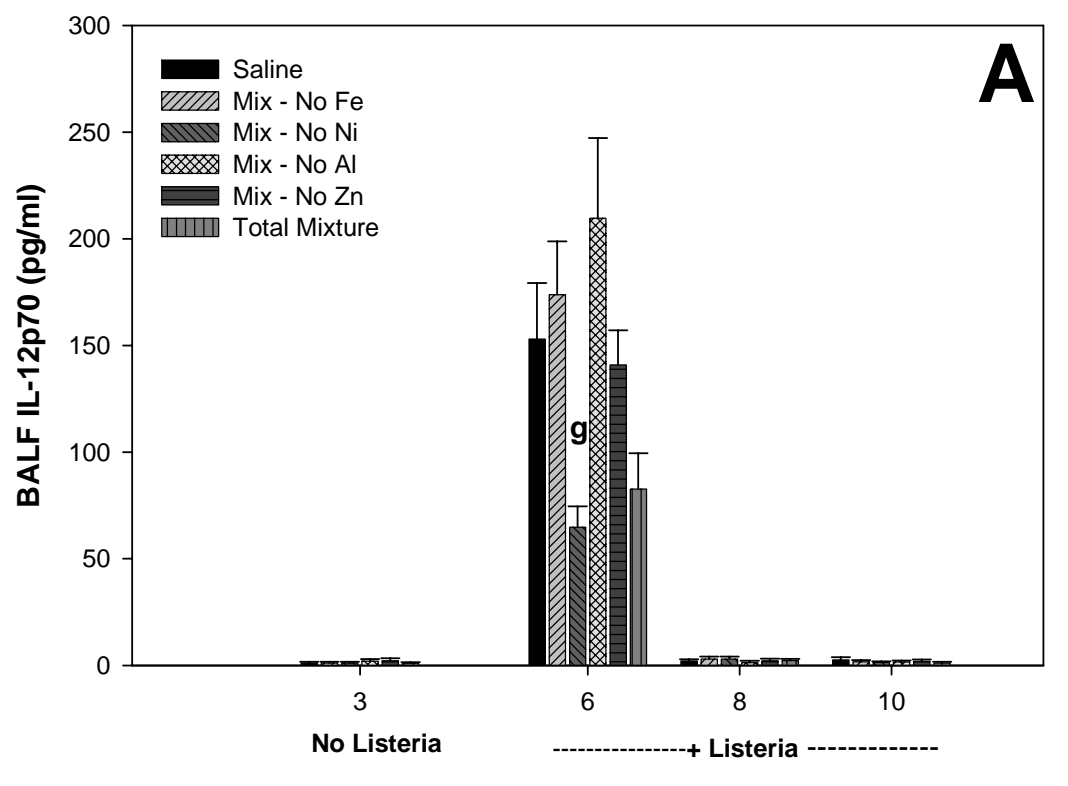

Time (Days Post- Metal Instillation)

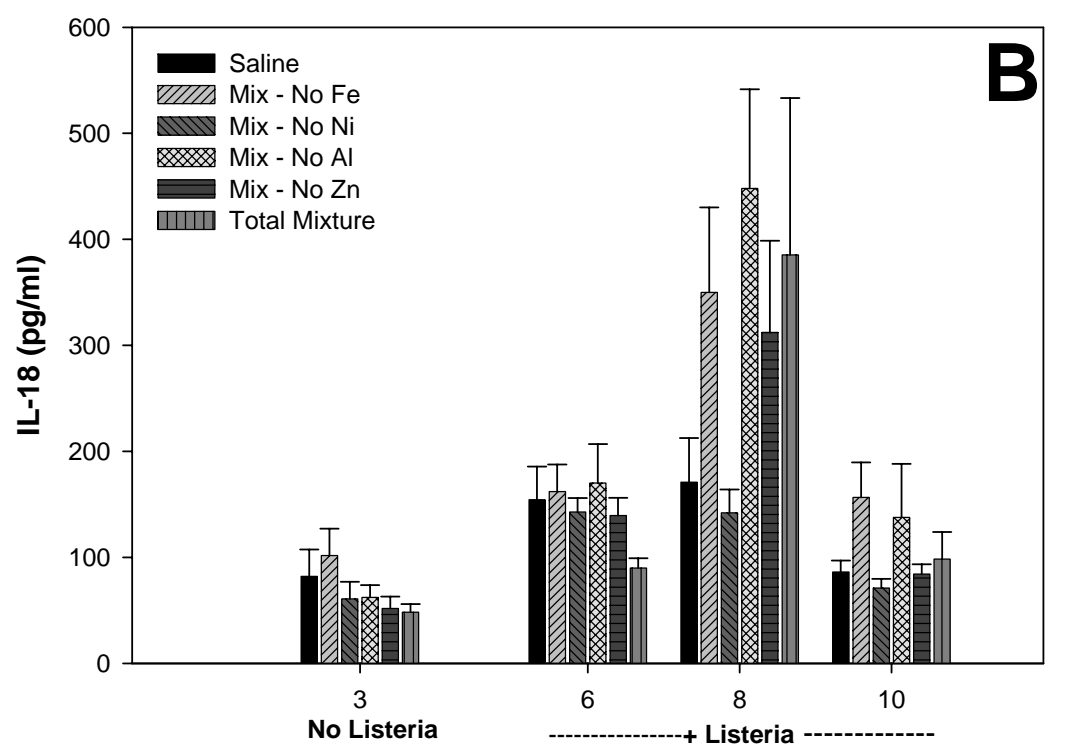

Time (Days Post- Metal Instillation)

Figure 3.2.9. Cytokines involved in directing the appropriate $\mathrm{T}$ cell response to $L$. monocytogenes, IL-12p70 (A) and IL-18 (B), in the BAL fluid of rats that were preexposed to soluble metal mixtures, the total metal mixture, or saline 3 days prior to intratracheal inoculation with $L$. monocytogenes. Values are means $\pm \mathrm{SE} ;{ }^{\mathbf{g}}$ significantly different from Saline, Mix - No Fe, and Mix - No Al groups $(\mathrm{p}<0.05)$. 

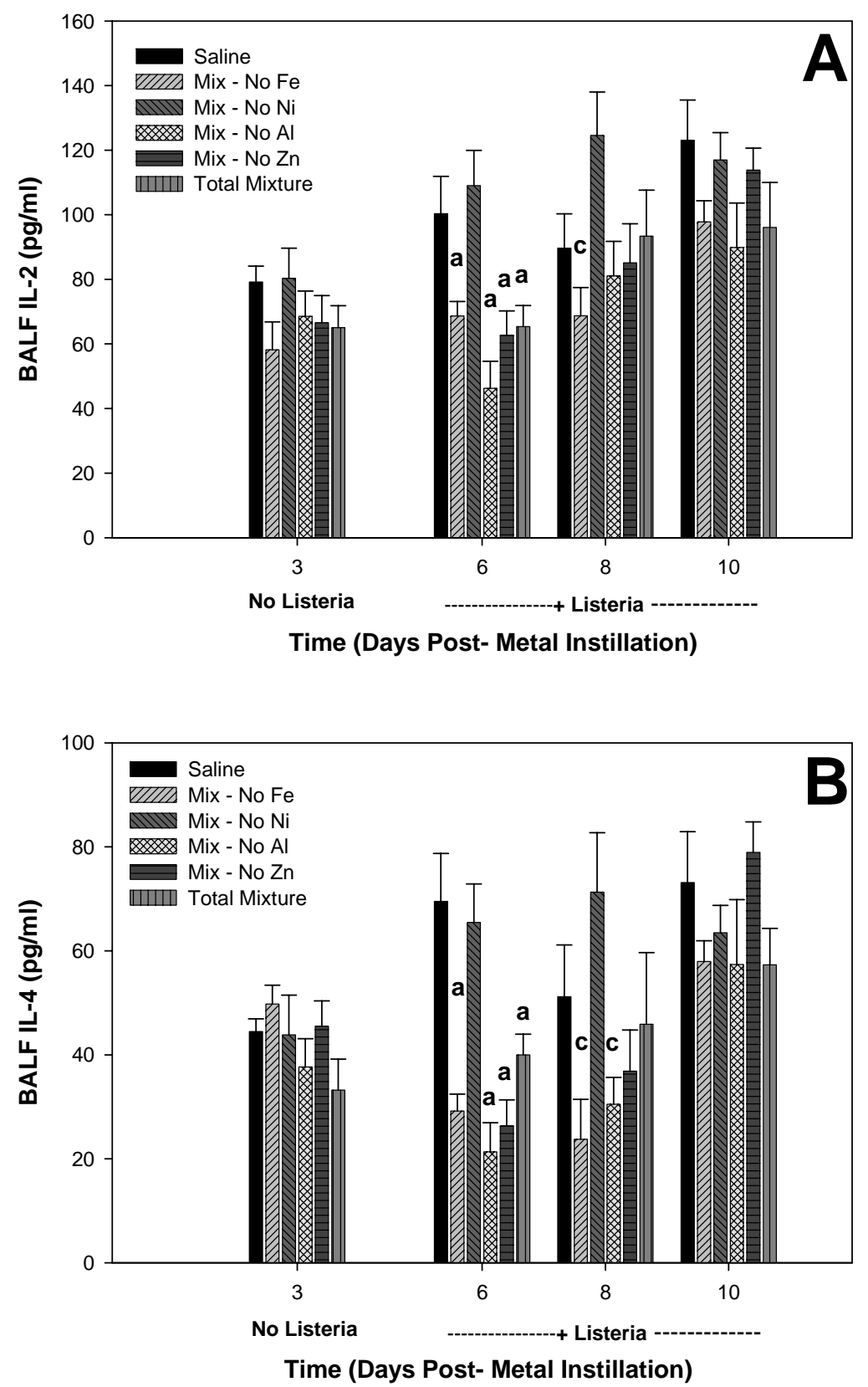

Figure 3.2.10. T cell cytokines, IL-2 (A) and IL-4 (B), in the BAL fluid of rats that were pre-exposed to soluble metal mixtures, the total metal mixture, or saline 3 days prior to intratracheal inoculation with $L$. monocytogenes. Values are means $\pm \mathrm{SE} ;{ }^{\mathbf{a}}$ significantly different from Saline and Mix - No Ni groups; ${ }^{c}$ significantly different from Mix - No Ni group $(\mathrm{p}<0.05)$. 


\section{Table 3.2.1. Metal Concentrations of Treatment Groups}

\begin{tabular}{ccccccc} 
& \multicolumn{6}{c}{ Treatment Groups } \\
\cline { 2 - 7 } Metal & Saline & Mix - No Fe & Mix - No Ni & Mix - No Al & Mix - No Zn & Total Mixture \\
\hline Soluble Fe & 0 & 0 & $37.2 \mu \mathrm{g}$ & $37.2 \mu \mathrm{g}$ & $37.2 \mu \mathrm{g}$ & $37.2 \mu \mathrm{g}$ \\
\hline Soluble Ni & 0 & $55.7 \mu \mathrm{g}$ & 0 & $55.7 \mu \mathrm{g}$ & $55.7 \mu \mathrm{g}$ & $55.7 \mu \mathrm{g}$ \\
Soluble Al & 0 & $46.6 \mu \mathrm{g}$ & $46.6 \mu \mathrm{g}$ & 0 & $46.6 \mu \mathrm{g}$ & $46.6 \mu \mathrm{g}$ \\
Soluble Zn & 0 & $8.69 \mu \mathrm{g}$ & $8.69 \mu \mathrm{g}$ & $8.69 \mu \mathrm{g}$ & 0 & $8.69 \mu \mathrm{g}$
\end{tabular}

Table 3.2.1. Quantities of soluble metals present in the $0.3 \mathrm{~mL}$ instillation volume of sterile PBS (vehicle) in each treatment group. The amounts of each metal used were equivalent to the quantity of the soluble metal as it was measured in $2 \mathrm{mg}$ of ROFA (Table 2, Study 1). Rats were intratracheally instilled with the metal mixtures on day 0 , followed by intratracheal inoculation with $5 \times 10^{4}$ L. monocytogenes on day 3 . 
Table 3.2.2. Bacterial Clearance

\begin{tabular}{ccccc} 
& \multicolumn{4}{c}{ CFUs LOG } \\
\cline { 2 - 5 } Treatment & $\begin{array}{c}\text { Day 3 } \\
\text { Initial Infection }\end{array}$ & Day 6 & Day 8 & Day 10 \\
\hline Saline & $4.8 \pm 0.01$ & $6.1 \pm 0.09$ & $4.3 \pm 0.31$ & $3.0 \pm 0.18$ \\
\hline Mix - No Fe & $4.8 \pm 0.01$ & $7.2 \pm 0.14^{\mathbf{a}}$ & $4.7 \pm 0.20$ & $3.1 \pm 0.17$ \\
\hline Mix - No Ni & $4.8 \pm 0.01$ & $6.1 \pm 0.08$ & $4.0 \pm 0.14$ & $2.2 \pm 0.52$ \\
\hline Mix - No Al & $4.8 \pm 0.01$ & $7.2 \pm 0.08^{\mathbf{a}}$ & $5.3 \pm 0.48$ & $3.2 \pm 0.59$ \\
\hline Mix - No Zn & $4.8 \pm 0.01$ & $7.1 \pm 0.09^{\mathbf{a}}$ & $4.8 \pm 0.30$ & $3.0 \pm 0.13$ \\
\hline Total Mixture & $4.8 \pm 0.01$ & $6.9 \pm 0.19^{\mathbf{a}}$ & $4.3 \pm 0.16$ & $3.3 \pm 0.23$ \\
\hline
\end{tabular}

Table 3.2.2. Number of bacterial CFUs in the left lungs of rats that were pre-exposed to soluble metal mixtures, the total metal mixture, or saline 3 days prior to intratracheal inoculation with $L$. monocytogenes. Values are means $\pm \mathrm{SE}$; ${ }^{\mathrm{a}}$ significantly different from Saline and Mix - No Ni groups ( $<<0.05)$. 


\section{Table 3.2.3. T/B Cell Ratio}

BAL T/B Cell Ratio

\begin{tabular}{ccccc}
\cline { 2 - 5 } Treatment & $\begin{array}{c}\text { Day 3 } \\
\text { Pre-Infection }\end{array}$ & Day 6 & Day 8 & Day 10 \\
\hline Saline & $0.793 \pm 0.174$ & $0.941 \pm 0.235$ & $1.97 \pm 0.228$ & $8.87 \pm 3.34$ \\
\hline Mix - No Fe & $1.01 \pm 0.293$ & $1.42 \pm 0.422$ & $3.00 \pm 0.321$ & $3.47 \pm 0.80$ \\
\hline Mix - No Ni & $1.03 \pm 0.426$ & $0.900 \pm 0.159$ & $1.88 \pm 0.330$ & $6.57 \pm 1.12$ \\
\hline Mix - No Al & $1.69 \pm 0.393$ & $0.973 \pm 0.397$ & $3.65 \pm 0.612$ & $6.68 \pm 1.97$ \\
\hline Mix - No Zn & $1.80 \pm 0.364$ & $0.956 \pm 0.168$ & $3.09 \pm 0.592$ & $4.97 \pm 0.589$ \\
\hline Total Mixture & $1.24 \pm 0.276$ & $0.523 \pm 0.193$ & $3.84 \pm 0.534$ & $5.33 \pm 0.803$ \\
\hline
\end{tabular}

Table 3.2.3. Ratio of $\mathrm{T}$ cells to B cells retrieved from the BAL of rats that were preexposed to soluble metal mixtures, the total metal mixture, or saline 3 days prior to intratracheal inoculation with L. monocytogenes. 
Table 3.2.4. CD4+/CD8+ T Cell Ratio

\begin{tabular}{ccccc} 
& \multicolumn{4}{c}{ BAL CD4+/CD8+ T Cell Ratio } \\
\cline { 2 - 5 } Treatment & $\begin{array}{c}\text { Day 3 } \\
\text { Pre-Infection }\end{array}$ & Day 6 & Day 8 & Day 10 \\
\hline Saline & $3.25 \pm 1.19$ & $0.901 \pm 0.106$ & $1.40 \pm 0.236$ & $1.91 \pm 0.273$ \\
\hline Mix - No Fe & $4.81 \pm 0.926$ & $3.32 \pm 1.14$ & $1.29 \pm 0.254$ & $1.68 \pm 0.371$ \\
\hline Mix - No Ni & $2.09 \pm 0.612$ & $2.28 \pm 0.618$ & $1.31 \pm 0.220$ & $1.94 \pm 0.316$ \\
\hline Mix - No Al & $2.53 \pm 0.922$ & $3.13 \pm 1.55$ & $1.60 \pm 0.200$ & $1.08 \pm 0.163$ \\
\hline Mix - No Zn & $2.93 \pm 0.552$ & $1.92 \pm 0.778$ & $1.70 \pm 0.632$ & $1.66 \pm 0.144$ \\
\hline Total Mixture & $3.46 \pm 0.995$ & $1.90 \pm 1.36$ & $1.80 \pm 0.500$ & $1.57 \pm 0.464$ \\
\hline
\end{tabular}

Table 3.2.4. Ratio of CD4+ $\mathrm{T}$ cells to $\mathrm{CD} 8+\mathrm{T}$ cells retrieved from the BAL of rats that were pre-exposed to soluble metal mixtures, the total metal mixture, or saline 3 days prior to intratracheal inoculation with $L$. monocytogenes. 


\section{References}

Abbey, D. E., Nishino, N., McDonnell, W. F., Burchette, R. J., Knutsen, S. F., Beeson, W. L., and Yang, J. X. 1999. Long-term inhalable particles and other air pollutants related to mortality in nonsmokers. Am. J. Respir. Crit. Care Med. 159:373-382.

Adkins, B., Jr., Richards, J. H., and Gardner, D. E. 1979. Enhancement of experimental respiratory infection following nickel inhalation. Environ. Res. 20: 33-42.

Akira, S., Hirano, T., Taga, T., and Kishimoto, T. 1990. Biology of multifunctional cytokines: IL-6 related molecules (IL-1 and TNF). FASEB J. 4:2860-2867.

American Thoracic Society, Committee of the Environmental and Occupational Health Assembly, Bascom, R., Bromberg, P. A., Costa, D. A., Devlin, R., Dockery, D. W., Frampton, M. W., Lambert, W., Samet, J. M., Speizer, F. E., and Utell, M. 1996. Health effects of outdoor air pollution. Part 1. Am. J. Respir. Crit. Care Med. 153:3-50.

Antonini, J. M., Roberts, J. R., Jernigan, M. R., Yang, H-M., Ma, J. Y., and Clarke, R. W. 2002. Residual oil fly ash increases susceptibility to infection and severely damages the lungs after pulmonary challenge with bacterial pathogen. Toxicol. Sci. 70: 110-119.

Aranyi, C., Miller, F. J., Andres, S., Ehrlich, R., Fenters, J., Gardner, D. E., and Waters, M. D. 1979. Cytotoxicity to alveolar macrophages of trace metals adsorbed onto fly ash. Environ. Res. 20: 14-23.

Aranyi, C., Bradof, J. N., Fenters, J. D., Graham, J. A., and Miller, F. J. 1981. Effects of inhalation or arsenic trioxide aerosols on the pulmonary defenses of mice. In "Heavy Metals in the Environment", pp. 450-453. Commission of the European Communities and the World Health Organization.

ATSDR: Agency for Toxic Substances and Disease Registry. 2005. Toxicological profile for nickel. Update (PB/2006-100005). U. S. Department of Health and Human Services, Atlanta, GA.

Berg, R. E., Cordes, C. J., and Forman, J. 2002. Contribution of CD8+ T cells to innate immunity: IFN- $\gamma$ secretion induced by IL-12 and IL-18. Eur. J. Immunol. 32: 2807-2816.

Billiau, A. 1996. Interferon-gamma: biology and role in pathogenesis. Adv. Immunol. 62: 61-130.

Blesson, S., Thiery, J., Gaudin, C., Stancou, R., Kolb, J-P., Moreau, J-L., Theze, J., Mami-Chouaib, F., and Chouaib S. 2002. Analysis of the mechanism of human cytotoxic T lymphocyte response inhibition by NO. Internat. Immunol. 14: 1169-1178.

Borish, L., Rosenbaum, R., Albury, L., and Clark, S. 1989. Activation of neutrophils by recombinant interleukin 6. Cell. Immunol. 121: 280-289. 
Brain, J. D., Knudson, D. E., Sorokin, S. P., and Davis, M. A. 1976. Pulmonary distribution of particles given by intratracheal instillation or by aerosol inhalation. Environ. Res. 11:13-33.

Burnett, R. T., Brook, J., Dann, T., Delocla, C., Philips, O., Cakmak, S., Goldberg, M. s., and Krewski, D. 2000. Association between particulate- and gas-phase components of urban air pollution and daily mortality in eight Canadian cities. Inhal. Toxicol. 12(Suppl. 1): $15-39$.

Calderon-Garciduenas, L., Devlin, R. B., and Miller, F. J. 2000. Respiratory tract pathology and cytokine imbalance in clinically healthy children chronically and sequentially exposed to air pollutants. Med. Hypth. 55: 373-378.

Camner, P., Casarett-Bruce, M., Curdstedt, T., Jarstrand, C., Wiernik, A., Johansson, A., Lundborg, M., and Robertson, B. 1984. Toxicology of nickel. In: Nickel in the human environment, IARC Scientific Publication No. 53. ed. Sunderman, F. W., Jr.International Agency for Research on Cancer: Lyon, France. Pp. 267-276.

Castranova, V., Bowman, L., Miles, P. R., and Reasor, J. M. 1980a. Toxicity of metal ions to alveolar macrophages. Am. J. Ind. Med. 1: 349-357.

Castranova, V., Bowman, L., Reasor, J. M., and Miles, P. R. 1980b. Effects of heavy metal ions on selected oxidative metabolic processes in rat alveolar macrophages. Toxicol, Appl. Pharmacol. 53: 14-23.

Castranova, V., Jones, T., Barger, M., Afshari, A., and Frazer, D. J. 1990. Pulmonary responses of guinea pigs to consecutive exposures to cotton dust. Proc. $14^{\text {th }}$ Cotton Dust Research Conference, eds. Jacobs, R. R., Wakelyn, P. J., and Domelsmith, L. N. pp 131135. Memphis, TN: National Cotton Council.

Chan, S. H., Kobayashi, M., Santoli, D., Perussia, B., and Trinchieri, G. 1992.

Mechanisms of IFN-gamma induction by natural killer cell stimulatory factor (NKSF/IL12). Role of transcription and mRNA stability in the synergistic interaction between NKSF and IL-2. J. Immunol. 148: 92-98.

Chen, C. Y., Wang, Y. F., Huang, W. R., and Huang, Y. T. 2003a. Nickel induces oxidative stress and genotoxicity in human lymphocytes. Toxicol. Appl. Pharmacol. 189: 153-159.

Chen, C. Y., Wang, Y. F., Lin, Y. H., and Yen, S. F. 2003b. Nickel-induced oxidative stress and effect of antioxidants in human lymphocytes. Arch. Toxicol. 77: 123-130.

Clarke, R. W., Catalano, P. J., Koutrakis, P., Murthy, G. G. K., Sioutas, C., Paulauskis, J., Coull, B., Ferguson, S., and Godleski, J. J. 1999. Urban air particulate inhalation alters 
pulmonary function and induces pulmonary inflammation in a rodent model of bronchitis. Inhal. Toxicol. 11: 637-656.

Cohen, M. D. 2004. Pulmonary immunotoxicology of select metals: aluminum, arsenic, cadmium, chromium, copper, manganese, nickel, vanadium, and zinc. J. Immunotoxicol. 1: 39-69.

Dalrymple, S. A., Lucian, L. A., Slattery, R., McNeil, T., Aud, D. M., Fuchino, S., Lee, F., and Murray, R. 1995. Interleukin-6-deficient mice are highly susceptible to Listeria monocytogenes infection: correlation with inefficient neutrophilia. Infect. Immun.. 63: 2262-2268.

Daniels, M. J., Menache, M. G., Burleson, G. R., Graham, J. A., and Selgrade, M. J. 1987. Effects of $\mathrm{NiCl}_{2}$ and $\mathrm{CdCl}_{2}$ on susceptibility to murine cytomegalovirus and virusaugmented natural killer cell and interferon responses. Fund. Appl. Toxicol. 8: 443-453.

De Smedt, A. C., van den Heuvel, R. L., Zwi Berneman, N., and Schoeters, G. E. 2001. Modulation of phenotype, cytokine production, and stimulatory function of CD34+derived DC by $\mathrm{NiCl}_{2}$ and SDS. Toxciol. In Vitro. 15: 319-325.

Djeu, J. Y., Liu, J. H., Wei, S., Rui, H., Pearson, C. A., Leonard, W. J., and Blanchard, D. K. 1993. Function associated with IL-2 receptor-beta on human neutrophils. Mechanism of activation of antifungal activity against Candida albicans by IL-2. J. Immunol. 150: 960-970.

Dockery, D. W., Pope III, C. A., Xu, X., Spengler, J. D., Ware, J. H., Fay, M. E., Ferris, B. G., and Speizer, F. E. 1993. An association between air pollution and mortality in six U.S. cities. N. Engl. J. Med. 329:1753-1759.

Dreher, K. L., Jaskot, R. H., Lehmann, J. R., Richards, J. H., McGee, J. K., Ghio, A. J., and Costa, D. L. 1997. Soluble transition metals mediate residual oil fly ash-induced acute lung injury. J. Toxicol. Environ. Health. 50:285-305.

Drummond, J. G., Aranyi, C., Schiff, L. J., Fenters, J. D., and Graham, J. A. 1986. Comparative study of various methods used for determining health effects of inhaled sulfates. Environ. Res. 41: 514-528.

Ehrlich, R., Findlay, J. C., and Gardner, D. E. 1978. Susceptibility to bacterial pneumonia of animals exposed to sulfates. Toxicol. Lett.1: 325-330.

Ehrlich, R. 1980. Interactions between environmental pollutants and respiratory infections. Environ. Health Perspect. 35: 89-100. 
Englert, N. 2004. Fine particles and human health - a review of epidemiological studies. Toxicol. Let. 149:235-242.

Espinoza-Delgado, I., Bosco, M. C., Musso, T., Gusella, G. L., Longo, D. L., and Varesio, L. 1995. Interleukin-2 and human monocyte activation. J. Leukoc. Biol. 57: 139.

Ford, H. R., Hoffman, R. A., Wang, S., and Simmons, R. L. 1991. Induction of cytotoxic T lymphocyte development from murine thymocytes by IL-1 and IL-6. J. Pediatr. Surg. 26: 397-400.

Ghio, A. J., Kim, C., and Devlin, R. B. 2000. Concentrated ambient particles induce mild pulmonary inflammation in healthy volunteers. Am. J. Respir. Crit. Care Med. 162: 981998.

Ghio, A. J. 2004. Biological effects of Utah Valley ambient air particles in humans: a review. J. Aerosol Med. 17: 157-164.

Goldstein, E., Lippert, W., Chang, D. P., and Tarkington, B. 1979. Effect of near ambient exposures to sulfur dioxide and ferrous sulfate particles on murine pulmonary defense mechanisms. Arch. Environ. Health. 34: 424-431.

Goutet, M., Ban, M., and Binet, S. 2000. Effects of nickel sulfate on pulmonary natural immunity in Wistar rats. Toxicol. 145: 15-26.

Green, L.C., Wagner, C. A., Glogowski, J., Skipper, P.L., Wishnok, J. S., and Tannenbaum, S. R. 1982. Analysis of nitrate, nitrite, and $\left[{ }^{15} \mathrm{~N}\right]$ nitrate in biological fluids. Anal. Biochem. 126: 131-138.

Haley, P. J., Shopp, G. M., Benson, J. M., Cheng, Y. S., Bice, D. E., Luster, M. I., Dunnick, J. K., and Hobbs, C. H. 1990. The immuntoxicity of three nickel compounds following 13-week inhalation exposure in the mouse. Fund. Appl. Toxicol. 15: 476-487.

Harkin, A., Hynes, M. J., Masterson, E., Kelly, J. P., O’Donnell., J. M., and Conner, T. J. 2003. A toxicokinetic study of nickel-induced immunosuppression in rats.

Immunopharmacol. Immunotoxicol. 25: 655-670.

Hatch, G. E., Slade, R., Boykin, E., Hu, P. C., Miller, F. J., and Gardner, D. E. 1981. Correlation of effects of inhaled versus intratracheally-injected metals on susceptibility to respiratory infection in mice. Am. Rev. Respir. Dis. 124: 167-173.

Hatch, G. E., Boykin, E., Graham, J. A., Lewtas, J., Pott, F., Loud, K., and Mumfor, J. L. 1985. Inhalable particles and pulmonary host defense: In vivo and in vitro effects of ambient air and combustion particles. Environ. Res. 36:67-80. 
Henney, C. S., Kuribayashi, K., Kern, D. E., and Gillis, S. 1981. Interleukin-2 augments natural killer cell activity. Nature. 291: 335-8.

Hertz-Picciotto, I., Herr, C. E., Yap, P. S., Dostal, M., Shumway, R. H. Ashwood, P., Lipsett, M., Joad, J. P., Pinkerton, K. E., and Sram, R. J. 2005. Air pollution and lymphocyte phenotype proportions in cord blood. Environ. Health Perspect. 113: 13911398.

Hirano, T., Akira, S., Taga, T, and Kishimoto, T. 1990. Biological and clinical aspects of interleukin 6. Immunol. Today. 11: 443-449.

Hoffman, R. A., Mahidhara, R. S., Wolf-Johnston, A. S., Lu, L., Thomsaon, A. W., and Simmons, R. L. 2002. Differential modulation of CD4 and CD8 T cell proliferation by induction of nitric oxide synthase in antigen presenting cells. Transplantation. 74:836845.

Hsieh, C. S., Macatonia, S. E., Tripp, C. S., Wolf, S. F., O'Garra, A., and Murphy, K. M. 1993. Development of $\mathrm{T}_{\mathrm{H}} 1 \mathrm{CD} 4+\mathrm{T}$ cells through IL-12 produced by Listeria-induced macrophages. Science. 260: 547-9.

Johansson, A.,Curstedt, T., Jarstrand, C. and Camner, P. 1992. Alveolar macrophages and lung lesions after combined exposure to nickel, cobalt, and trivalent chromium. Environ. Health Perspect. 97: 215-219.

Kodavanti, U. P. Jaskot, R. H., Costa, D. L., and Dreher, K. L. 1997. Pulmonary proinflammatory gene induction following acute exposure to residual oil fly ash: roles of particle-associated metals. Inhal. Toxicol. 9: 679-701.

Kodavanti, U. P., Hauser, R., Christiani, D. C., Meng, Z. H., McGee, J., Ledbetter, A., Richards, J., and Costa, D. L. 1998. Pulmonary responses to oil fly ash particles in the rat differ by virtue of their specific soluble metals. Toxicol. Sci. 43:204-212.

Kodavanti, U. P., Mebane, R., Ledbetter, A., Krantz, T., McGee, J., Jackson, M. C., Walsh, L., Hilliard, H., Chen, B. Y., Richards, J., and Costa, D. L. 2000. Variable pulmonary responses from exposure to concentrated ambient air particles in a rat model of bronchitits. Toxicol. Sci. 54: 441-451.

Kopf, M., Baumann, H., Freer, G., Freudenberg, M., Lamers, M., Kishimoto, T., Zinkernagel, R., Bluethmann, H., and Kohler, G. 1994. Impaired immune and acutephase responses in interleukin-6-deficient mice. Nature. 368: 339-342.

Laden, F., Neas, L. M., Dockery, D. W., and Schwartz, J. 2000. Association of fine particulate matter from different sources with daily mortality in six U. S. cities. Environ. Health. Perspect. 108: 941-947. 
Leonardi, G. S., Houthuijs, D., Steerenberg, P. A., Fletcher, T., Armstrong, B., and Antova, T. 2000. Immune biomarkers in relation to exposure to particulate matter: a cross-sectional survey in 17 cities in central Europe. Inhal. Toxicol.12(Suppl 4): 1-14.

Lotz, M., Jirik, F., Kabouridis, R. Tsoukas, C., Hirano, T., Kishimoto, T., and Carson, D. A. 1988. B cell stimulating factor/interleukin 6 is a costimulant for human thymocytes and T lymphocytes. J. Exp. Med. 167: 1253-1258.

Manome, H., Aiba, S., and Tagami, H. 1999. Simple chemicals can induce maturation and apoptosis of dendritic cells. Immunol.98: 481-490.

Minami, Y., Kono, T., Miyazaki, T., and Taniguchi, T. 1993. The IL-2 receptor complex: its structure, function, and target genes. Ann. Rev. Immunol. 11: 245-68.

Moore, K. W., de Waal Malefyt, R., Coffman, R. L., and O'Garra, A. 2001. Interleukin10 and the interleukin-10 receptor. Annu. Rev. Immunol.19:683-765.

Mosmann, T. R., and Sad, S. 1996. The expanding universe of T-cell subsets: Th1, Th2, and more. Immunol. Today. 17:138-146.

Naume, B., Johnsen, A. C., Espevik, T., and Sundan, A. 1993. Gene expression of cytokines and cytokine receptors from highly purified CD56+ natural killer cells stimulated with interleukin-2, interleukin-7, and interleukin-12. Eur. J. Immunol. 23: 1831-1838.

Neighbors, M., Xu, X., Barrat, F. J., Ruuls, S. R., Churakova, T. Debets, R., Bazan, J. F., Kastelein, R. A., Abrams, J. S., and O'Garra, A. 2001. A critical role for interleukin 18 in primary and memory effector responses to Listeria monocytogenes that extends beyond its effects on interferon $\gamma$ production. J. Exp. Med. 194: 343-354.

NTP. 1996a. NTP technical report on the toxicology and carcinogenesis studies of nickel oxide (CAS No. 1313-99-1) in F344/N rats and B6C3F1 mice (inhalation studies). NTPTRS No. 451. Research Triangle Park, NC: U. S. Department of Health and Human Services, Public Health Service, National Institute of Health.

NTP. 1996b. NTP technical report on the toxicology and carcinogenesis studies of nickel subsulfide (CAS No. 12035-72-2) in F344/N rats and B6C3F1 mice (inhalation studies). NTP-TRS No. 453. Research Triangle Park, NC: U. S. Department of Health and Human Services, Public Health Service, National Institute of Health.

NTP. 1996c. NTP technical report on the toxicology and carcinogenesis studies of nickel sulfate hexahydrate (CAS No. 10101-97-0) in F344/N rats and B6C3F1 mice (inhalation studies). NTP-TRS No. 454. Research Triangle Park, NC: U. S. Department of Health and Human Services, Public Health Service, National Institute of Health. 
Okamura, H., Tsutsi, H., Komatsu, T., Yutsudo, M., Hakura, A., Tanimoto, T., Torigoe, K., Okura, T., Nukada, Y., Hattori, K., Akita, K., Namba, M., Tanabe, F., Konishi, K., Fukuda, S., and Kurimoto, M. 1995. Cloning of a new cytokine that induces IFN-gamma production by T cells. Nature. 378:88-91.

Omara, F. O., Brousseau, P., Blakely, B.,R., and Fournier, M. 1998. Iron, zinc, and copper. In: Immunotoxicology of environmental and Occupational Metals. Zelikoff, J. T., and Thomas, P. T., eds. Taylor and Francis, London, England. pp.163-194.

Pamer, E. G. 2004. Immune responses to Listeria monocytogenes. Nature Rev. 4:812823.

Paul, W. E., and Seder, R. A. 1994. Lymphocyte responses and cytokines. Cell. 76:241251.

Pope III, C. A., Dockery, D. W., and Schwartz, J. 1995. Review of epidemiological evidence of health effects of particulate air pollution. Inhal. Toxicol. 7:1-18.

Pope III, C. A., Burnett, R. T., Thurston, G. D., Thun, M. J., Calle, E. E., Krewski, D., and Godleski, J. J. 2004. Cardiovascular mortality and long-term exposure to particulate air pollution: epidemiological evidence of general pathophysiological pathways of disease. Circ. 109: 71-77.

Renauld, J. C., Vink, A., and Van Snick, J. 1989. Accessory signals in murine cytolytic T cell responses: dual requirement for IL-1 and IL-6. J. Immunol.143: 1894-1898.

Salnikow, K., Li, X., and Lippmann, M. 2004. Effect of nickel and iron co-exposure on human lung cells. Toxicol. Appl. Pharmacol. 196: 258-265.

Samet, J. M., Zeger, S. L., Dominici, F., Curriero, F., Coursac, I. Dockery, D. W., Schwartz, J., and Zanobetti, A. 2000a. The national morbidity, mortality, and air pollution study. Part II: Morbidity and mortality from air pollution in the United States. Res. Rep. Health Eff. Inst. 94:5-79.

Samet, J. M., Dominici, F., Curriero, F. C., Coursac, I., and Zeger, S. L. 2000b. Fine particulate air pollution and mortality in 20 U. S. cities, 1987-1994. N. Engl. J. Med. 343: 1742-1749.

Schaumann, F., Borm, P. J. A., Herbrich, A., Knoch, J., Pitz, M., Schins, R. P. F., Luettig, B., Hohlfeld, J. M., Heinrich, J., and Krug, N. 2004. Metal-rich ambient particles (particulate matter 2.5 ) cause airway inflammation in healthy subjects. Am. J. Respir. Crit. Care Med. 170: 898-903.

Schluter, T., Berg, I., Dorger, M., and Gercken, G. 1995. Effect of heavy metal ions on the release of reactive oxygen intermediates by bovine alveolar macrophages. Toxicol. 98: 47-55. 
Schwartz, J. 1994. What are people dying from on high pollution days? Environ. Res. 64:26-35.

Seder, R. A., and Gazzinelli, R. T. 1999. Cytokines are critical in linking the innate and adaptive immune responses to bacterial, fungal, and parasitic infection. Adv. Intern. Med. 44: 353-88.

Sherwood, R. L., Lippert, W. E., Goldstein, E., and Tarkington, B. 1981. Effect of ferrous sulfate aerosols and nitrogen dioxide on murine pulmonary defense. Arch. Environ. Health. 36: 130-135.

Skachkova, M. A., Skachkov, M. V., Smolyagin, A. I., Boev, V. M., Vereshchagin, N. N., Mikhailova, I. V., Popova, E. V., and Skvortsov, V. O. 2001. Immune and interferon status in schoolchildren living in cities with different anthropologic load. Bull. Exp. Biol. Med. 4: 371-373.

Skornik, W. A., and Brain, J. D. 1983. Relative toxicity of inhaled metal sulfate salts for pulmonary macrophages. Am. Rev. Respir. Dis. 126: 297-303.

Smialowicz, R. J. 1998. Nickel. In: Immunotoxicology of environmental and Occupational Metals. Zelikoff, J. T., and Thomas, P. T., eds. Taylor and Francis, London, England. pp.163-194.

Smialowicz, R. J., Rogers, R. R., Riddle, M. M., and Stott, G. A. 1984. Immunologic effects of nickel. I. Suppression of cellular and humoral immunity. Environ. Res. 33: 413427.

Smialowicz, R. J., Rogers, R. R., Riddle, M. M., Gardner, R. J., Rowe, D. G., and Luebke, R. W. 1985. Immunologic effects of nickel. II. Suppression of natural killer (NK) cell activity. Environ. Res. 36: 56-66.

Smialowicz, R. J., Rogers, R. R., Riddle, M. M., Luebke, R. W., Fogelson, L. D., nad Rowe, D. G. 1987. Effects of manganese, calcium, magnesium, and zinc on nickelinduced suppression of murine natural killer cell activity. J. Toxicol. Environ. Health. 20: $67-80$.

Stoll, S., Jonuleit, H, Schmitt, E., Muller, G., Yarnauchi, H., Kurimoto, M., Knop, J., and Enk, A. H. 1998. Production of functional IL-18 by different subtypes of murine and human dendritic cells (DC): DC-derived IL-18 enhances IL-independent Th1 development. Eur. J. Immunol. 28:3231-3239.

Sunderman, F. W. Jr., Hopfer, S. M., Lin, S. M., Plowman, M. C., Stojanivic, T., Wong, S. H., Zaharia, O., and Ziebka, L. 1989. Toxicity to alveolar macrophages in rats following parenteral injection of nickel chloride. Toxicol. Appl. Pharmacol. 100: 107118. 
Swain, S. L. 2001. Interleukin-18: Tipping the balance towards a T helper cell 1 response. J. Exp. Med. 194: F11-F14.

Trinchieri, G. 1995. Interleukin 12: A proinflammatory cytokine with immunoregulatory functions that bridge innate resistance and antigen-specific adaptive immunity. Annu. Rev. Immunol. 13: 251-276.

Tsutsui, H., Nakanishi, K., Matsui, K., Higashino, K., Okamura, H., Miyazawa, Y., and Kaneda, K. 1996. IFN-gamma-inducing factor up-regulates Fas ligand-mediated cytotoxic activity of murine natural killer cell clones. J. Immunol. 157: 3967-3973.

Van der Veen, R. C., Dietlin, T. A., Pen, L., and Gray, J. D. 1999. Nitric oxide inhibits proliferation of T-helper 1 and 2 lymphocytes without reduction in cytokine secretion. Cell. Immunol. 193: 194-201.

Van der Veen, R. C., Dietlin, T. A., Gray, J. D., and Gilmore, W. 2000. Macrophagederived nitric oxide inhibits the proliferation of activated $\mathrm{T}$ helper cells and is induced during antigenic stimulation of resting T cells. Cell. Immunol. 199: 43-49.

Wozniak, K., and Blasiak, J. 2002. Free radicals-mediated induction of oxidized DNA bases and DNA-protein cross-links by nickel chloride. Mutat. Res. 514: 233-243.

Zelikoff, J. T., Schermerhorn, K. R., Fang, K., Cohen, M. D., and Schlesinger, R. B. 2002. A role for associated transition metals in the immunotoxicology of inhaled ambient particular matter. Environ. Health Perspect. 110 suppl 5: 871-875.

Zelikoff, J. T., Chen, L. C., Cohen, M. D., Fang, K., Gordon, T., Li, Y., Nadziejko, C., and Schlesinger, R. B. 2003. Effects of inhaled ambient particulate matter on pulmonary antimicrobial immune defense. Inhal. Toxicol. 15: 131-150. 


\section{GENERAL DISCUSSION}

Epidemiological studies have shown a correlation between PM exposure and elevated morbidity and mortality indicated by increased hospital emissions for both cardiovascular and respiratory diseases (Abbey et al., 1999; Dockery et al., 1993; Englert, 2004; Ghio, 2004; Pope et al., 1995; 2004; Samet et al., 2000a, 2000b; Schwartz, 1994). According to these studies, conditions regarding pulmonary effects include, but are not limited to, increased mortality from pneumonia and influenza, increased lung inflammation, and altered pulmonary host defense (ATS, 1996). Re-analysis of a large cohort epidemiological study by Pope et al. (2004) showed that the leading cause of mortality in response to increases in ambient air PM in the category of pulmonary conditions was infection, including pneumonia and influenza. Population studies in industrialized areas have also revealed that in regions with high anthropologic PM loads, particularly in the $\mathrm{PM}_{2.5}$ range, whole blood and serum levels of lymphocytes (NK cells, $\mathrm{B}$ and $\mathrm{T}$ cell subsets), immunoglobulin (Ig), and cytokines are significantly altered (Calderon-Garciduenas et al., 2000; Hertz-Picciotto et al., 2005; Leonardi et al., 2000; Skachkova et al., 2001). Human exposure studies have also demonstrated that PM that is high in metals can increase leukocytes in the lung, as well as increase oxidant and cytokine production by phagocytes, i.e., cellular processes that are important in pulmonary responses to pathogens (Ghio, 2004; Ghio et al., 2000; Schaumann et al., 2004). In addition, animal studies have shown that a number of air pollutants, including diesel, (Hahon et al., 1985; Harrod et al., 2003; 2005; Yang et al., 2001; Yin et al., 2002; 2003), CAPs (Zelikoff et al., 2003), certain types of CFA (Hatch et al., 1985), and ROFA (Antonini et al., 2002; Hatch et al., 1985) can increase susceptibility to pulmonary 
infection. Particulates that were most potent at altering infectivity were the samples that were smallest in size and/or highest in soluble metal content, such as ROFA (Hatch et al., 1985).

ROFA is a complex mixture of soluble and insoluble metals, sulfates, and acids complexed to an insoluble particulate carbon core, generated from the combustion of fossil fuels (Antonini et al., 2004a; Ghio et al., 2002a; Kodavanti et al., 1998; Schroeder et al., 1987). Metals present in ROFA can include Fe, V, Ni, Al, Zn, Ca, and to a lesser extent, $\mathrm{Cu}, \mathrm{Co}, \mathrm{Cd}, \mathrm{Ba}, \mathrm{Be}, \mathrm{Mn}, \mathrm{Cr}$, and Pb (Antonini et al., 2002; Ghio et al., 2002a; Kodavanti et al., 1998). The objectives of the current study were; 1) determine which component of ROFA (the soluble or insoluble metal constituent) was responsible for increased susceptibility to pulmonary infection, 2) to elucidate the mechanisms involved in susceptibility to infection, and 3) to determine what metal or metal combinations cause these effects. A number of studies have indicated that the soluble fraction of ROFA is largely responsible for increased inflammation and lung injury (Dreher et al., 1997; Kodavanti et al., 1998), increased airway hypersensitivity (Gavett et al., 1997; Lambert et al., 2000), elevated oxidative stress (Goldsmith et al., 1998; Huang et al., 2003a; Kadiiska et al., 1997), altered cytokine expression (Carter et al., 1997; Dye et al., 1999; Lambert et al., 2000), and altered signal transduction and gene expression (Kodavanti et al., 1997; Nadadur et al., 2002; Samet et al., 1997). Based on this, the hypothesis for the current study was that the soluble metals in ROFA would likely be responsible for the increase in susceptibility to infection, and, given the composition of ROFA and the numerous studies that have examined the suppressive effects of $\mathrm{Ni}$ on cells involved in innate and adaptive 
immunity (ATSDR, 2005a), that Ni would be the primary metal involved in inducing the susceptibility to pulmonary infection.

In study 1, the ROFA sample used in the current investigation was determined to be composed primarily of $\mathrm{Fe}, \mathrm{V}, \mathrm{Ni}, \mathrm{Ca}, \mathrm{Al}$, and $\mathrm{Zn}$. The soluble metal fraction contained significant amounts of $\mathrm{Fe}, \mathrm{Al}, \mathrm{Ni}$, and $\mathrm{Zn}$, whereas the majority of the $\mathrm{V}$ was insoluble. Study 1 also clearly demonstrated that the soluble metals in ROFA were solely responsible for the decreased clearance of $L$. monocytogenes from rat lungs that had been exposed to the total ROFA sample. In addition, rats that received the insoluble metals or the sample where the soluble metals had been extracted were able to clear the infection with the same efficacy as the control rats. Alterations in bacterial clearance and body weight were reproduced by exposure to an artificial mixture of soluble $\mathrm{Al}, \mathrm{Fe}, \mathrm{Ni}$, and $\mathrm{Zn}$ as shown in study 3 , although the infectivity was not altered quite as strongly as it was with the soluble ROFA sample. One explanation for this is that the concentrations of the soluble metals were likely underestimated due to a small loss of a portion of the soluble sample of ROFA in the separation process. Greater quantities of these soluble metals may have further decreased bacterial clearance at later time points in the course of the infection, as was observed the soluble ROFA sample. Of all the metal constituents examined, only soluble $\mathrm{Ni}$, alone or in a combination with other metals, was capable of increasing susceptibility to infection, and the increase was comparable to that observed with the artificial metal mixture (Study 3). Therefore, it is likely that soluble $\mathrm{Ni}$, at the concentration that it exists in ROFA, is the primary metal constituent responsible for the increase in infectivity in rats exposed to ROFA. 
Soluble Ni also appears to account for the increased lung injury and inflammation, prior to and after infection, that was observed with the soluble portion of ROFA in study 2. On day 3, prior to infection, soluble Ni alone or in combination with other metals caused the same degree of lung injury and permeability as was shown with soluble ROFA, indicated by increased BAL levels of LDH and albumin. This is consistent with the findings of other investigators that show that soluble Ni in ROFA can induce lung injury at the concentrations present in the ROFA used (Dreher et al., 1997; Kodavanti et al., 1998). They have shown that the injury caused by Ni can be attenuated in the presence of other soluble metals, such as Fe, indicating possible antagonistic effects of metals in mixtures. Post-infection, soluble Ni also appeared to account for the increase in lung injury, as well as increased inflammation, indicated by increased BAL levels of LDH and albumin on days 6 and 8, and significant influx of macrophages and neutrophils into the airspaces on days 6,8 , and 10 .

The soluble metals that exist in ROFA are known to have a variety of effects on cells involved in the innate and adaptive immune response, as previously discussed. The immune response to L. monocytogenes, an intracellular bacteria that resides in the cytoplasm of cells, requires activation of innate immune cells (macrophages, neutrophils, DCs, and NK cells) as well as cells involved in cell-mediated immunity (CD8+ T cells and CD4+ $\mathrm{T}_{\mathrm{H}} 1$ cells) in order to effectively respond to the infection. The most significant results of pre-treatment with soluble ROFA, soluble $\mathrm{Ni}$, or mixtures containing soluble $\mathrm{Ni}$ were the apparent alterations on innate responses, as indicated by increased bacterial burden early after infection, and inhibition of $\mathrm{T}$ cell activity, demonstrated by reduced $\mathrm{T}$ cell cytokines in BAL. Increased bacterial burdens on day 6 indicate that treatment with 
soluble ROFA, soluble $\mathrm{Ni}$, or mixtures containing soluble Ni suppresses the activity of innate immune responses, despite an increased influx of phagocytes. Soluble Ni has been shown to inhibit AM phagocytic rates (ATSDR 2005a; Cohen et al., 2004). The soluble $\mathrm{Ni}$ in ROFA may be altering clearance of bacteria by suppressing phagocytosis and/or killing of bacteria by AMs. In addition, despite an overall increase in NO in the BALF of rats treated with the soluble portion of ROFA, NO production by AMs appeared to be reduced. In addition, IL-10, a cytokine shown to inhibit macrophage activity, was increased when compared to control animals. The IL-10 results were not replicated with soluble Ni alone or in a metal combination. Despite this, there was still a significant increase in bacterial burden on day 6; therefore, there is also likely to be a suppression of AM activity in these groups as well. As mentioned earlier, the level of infection in the groups containing soluble Ni did not persist as long as in the soluble ROFA-treated rats, possibly due to an underestimation of the concentration of metals in that sample. It is possible that higher concentrations of Ni may be required before noticeable alterations in IL-10 can be detected. NK cells, important in AM activation and killing of infected cells, are also known to be adversely affected by Ni (Smialowicz, 1998). However, data regarding their activity in this study are inconclusive. IFN- $\gamma$ levels in the BAL of rats were variable. In addition, NK cells are not the only producers of this cytokine. Alterations in NK cell activity may be better assessed in the lung tissue or lungassociated lymph nodes, or by examining cytokine production ex vivo or in vitro after exposure to soluble ROFA, $\mathrm{Ni}$, or metal combinations containing $\mathrm{Ni}$.

During the innate immune response, there were also significantly elevated levels of IL-6 and NO in the BALF of rats exposed to soluble ROFA or Ni on day 6. This 
elevation in IL-6 and NO may be due to the significant influx of neutrophils in these groups, indicating a severe inflammatory response. Elevated IL-6 is also indicative of an exacerbated acute phase response, and possibly contributed to the excessive lung injury observed in these groups on day 6. In addition, $\mathrm{NO}$ is known to suppress $\mathrm{T}$ cell proliferation (Blesson et al., 2002; Hoffman et al., 2002; van der Veen et al., 1999; 2000) and in some instances cytokine production (Blesson et al., 2002), and may be involved in inhibition of the adaptive response in the lung.

Soluble Ni associated with ROFA appeared to alter the adaptive immune response to L. monocytogenes infection as well. After infection, there was an overall increase in the number of $\mathrm{T}$ cells infiltrating the lungs of all treatment groups. Although $\mathrm{T}$ cell recruitment did not differ among treatment groups at the early time point post-infection (days 6 and 8), activity of T cells appeared to be inhibited in rats pre-exposed to soluble ROFA, Ni alone, or mixtures containing $\mathrm{Ni}$, as indicated by significantly lower levels of IL-2, and, to a lesser degree, IL-4. It is difficult to determine whether elevated NO levels, the metals themselves, or a combination of the two, are responsible for the effects on $\mathrm{T}$ cells. On day 3 , there is a reduction in IL-2 prior to infection in the rats pre-treated with soluble ROFA, in the absence of a significant increase in NO, indicating the metal portion may be exerting an effect. However, after infection, when NO levels are dramatically increased, there is a further decrease in IL-2 in the soluble ROFA group and all rats treated with soluble Ni. It is likely that the inhibition in $\mathrm{T}$ cell cytokine production is in part due to a combination of the inhibitory effects of soluble $\mathrm{Ni}$ on $\mathrm{T}$ cells along with the adverse effects of NO. Ex vivo examination of T cells from animals exposed to soluble Ni or the soluble ROFA sample in the presence or absence of NOS inhibitors, 
such as L-NMMA or L-NAME, would help to establish whether there is a role for NO in the inhibition of $\mathrm{T}$ cell function after exposure to mixtures containing soluble $\mathrm{Ni}$, or whether another mechanism is responsible. Interestingly, despite the increase in infection, the $\mathrm{T}$ cell responses and the NO response were not observed in the rats treated with the total ROFA sample prior to infection. Whether or not the presence of the particulate portion of the ROFA sample may be altering the activity or bioavailability of the soluble metals, or exerting effects to counteract those of the soluble portion remains to be determined.

Discrepancies also exist between the groups treated with the soluble ROFA sample, the soluble $\mathrm{Ni}$ alone, or the total ROFA sample in regards to cytokines involved in the signaling between the innate and adaptive immune responses. IL-12 and IL-18 are produced by macrophages and DCs and are involved in initiation of cell-mediated immunity. There were no significant differences in IL-12 in the soluble ROFA group or the artificial mixture after infection. However, IL-12 was increased in the rats treated soluble $\mathrm{Ni}$ alone on day 6 , but not in a metal combination with soluble Ni. In addition, IL-12 was significantly decreased in the total ROFA group on day 6. It is difficult to discern if interactions between the particulate portion and soluble portion of ROFA are responsible for the decrease in this cytokine; if there is a soluble metal that is counteracting the effects of soluble Ni on stimulation of IL-12; or which cell type these mixtures may be acting upon. Where one solution may be inhibiting AMs, the other may be stimulating DCs. In vitro studies on AMs and DCs after exposure to ROFA, the soluble portion of ROFA, or the soluble metal constituents may provide some insight as to the influence of these components on directing the adaptive immune response. In 
addition, experiments are currently underway to characterize the lymphocyte profiles and activity of lymphocytes in the lung-associated lymph nodes of animals from studies 2 and 3. Cell phenotype and cytokine production will provide more information as to the influence of the soluble metals in ROFA on differentiation of T cells early after infection, as well as the potential activity of NK cells and antigen presenting cells (primarily macrophages and DCs).

Although there may be some differences among various treatment groups, the results of these three studies indicate that the soluble metals in ROFA induced lung injury and inflammation, and altered both innate and adaptive immune responses in rats after infection with L. monocytogenes. The suppression of the innate immune response and inhibition of $\mathrm{T}$ cell function appeared to be highly dependent on soluble $\mathrm{Ni}$ at the concentration present in the soluble ROFA sample. Therefore, the metal composition of PM and the bioavailability of the metals in the PM may ultimately be the major determinants in the potential for adverse health effects, such as increased susceptibility to infection, for a given population. 


\section{GENERAL REFERENCES}

Abbey, D. E., Nishino, N., McDonnell, W. F., Burchette, R. J., Knutsen, S. F., Beeson, W. L., and Yang, J. X. 1999. Long-term inhalable particles and other air pollutants related to mortality in nonsmokers. Am. J. Respir. Crit. Care Med. 159:373-382.

Adkins, B., Jr., Richards, J. H., and Gardner, D. E. 1979. Enhancement of experimental respiratory infection following nickel inhalation. Environ. Res. 20: 33-42.

Adkins, B., Jr., Luginbuhl, G. H., Miller, F. J., and Gardner, D. E. 1980. Increased pulmonary susceptibility to streptococcal infection following inhalation of manganese oxide. Environ. Res. 23: 110-120.

Ameille, J., Brechot, J. M., Brochard, P., Capron, F., and Dore, M. F.1992. Occupational hypersensitivity pneumonitis in a smelter exposed to zinc fumes. Chest. 101(3): 862-3.

American Thoracic Society, Committee of the Environmental and Occupational Health Assembly, Bascom, R., Bromberg, P. A., Costa, D. A., Devlin, R., Dockery, D. W., Frampton, M. W., Lambert, W., Samet, J. M., Speizer, F. E., and Utell, M. 1996. Health effects of outdoor air pollution. Part 1. Am. J. Respir. Crit. Care Med. 153:3-50.

Antonini, J. M., Roberts, J. R., Jernigan, M. R., Yang, H. M., Ma, J. Y., and Clarke, R. W. 2002. Residual oil fly ash increases susceptibility to infection and severely damages the lungs after pulmonary challenge with bacterial pathogen. Toxicol. Sci. 70: 110-119.

Antonini, J. M., Taylor, M. D., Leonard, S. S., Lawryk, N. J., Shi, X., Clarke, R. W., and Roberts, J. R. 2004a. Metal composition and solubility determine lung toxicity by residual oil fly ash collected from different sites within a power plant. Mol. Cell. Biochem. 255: 257-265.

Antonini, J. M., Taylor, M. D., Millecchia, L., Bebout, A., R., and Roberts, J. R. 2004b. Suppression in lung defense responses after bacterial infection in rats pretreated with different welding fumes. Toxicol. Appl. Pharmacol. 200: 206-218.

Aranyi, C., Miller, F. J., Andres, S., Ehrlich, R., Fenters, J., Gardner, D. E., and Waters, M. D. 1979. Cytotoxicity to alveolar macrophages of trace metals adsorbed onto fly ash. Environ. Res. 20: 14-23.

Aranyi, C., Bradof, J. N., Fenters, J. D., Graham, J. A., and Miller, F. J. 1981. Effects of inhalation or arsenic trioxide aerosols on the pulmonary defenses of mice. In "Heavy Metals in the Environment", pp. 450-453. Commission of the European Communities and the World Health Organization.

Aranyi, C., Graf, J. L., O’Shea, W. J., Graham, J. A., and Miller, F. F. 1983. The effects of intratracheally-administered coarse mode particles on respiratory tract infection in mice. Toxicol. Lett. 19: 63-72. 
ATSDR: Agency for Toxic Substances and Disease Registry. 1992. Toxicological profile for vanadium. U. S. Department of Health and Human Services, Atlanta, GA.

ATSDR: Agency for Toxic Substances and Disease Registry. 1999. Toxicological profile for aluminum. Update (PB/99/166613). U. S. Department of Health and Human Services, Atlanta, GA.

ATSDR: Agency for Toxic Substances and Disease Registry. 2005a. Toxicological profile for nickel. Update (PB/2006-100005). U. S. Department of Health and Human Services, Atlanta, GA.

ATSDR: Agency for Toxic Substances and Disease Registry. 2005b. Toxicological profile for zinc. Update (PB/2006-100008). U. S. Department of Health and Human Services, Atlanta, GA.

Ball, B. R., Smith, K. R., Veranth, J. M., and Aust, A. E. 2000. Bioavailability of iron from coal fly ash: Mechanisms of mobilization and of biological effects. Inhal. Toxciol. 12 Suppl 4: 209-225.

Bancroft, G. J., Schreiber, R. D., and Unanue, E. R. 1991. Natural immunity: a T-cellindependent pathway of macrophage activation, defined in the SCID mouse. Immun. Rev. 124: 5-24.

Beck, B. D., Brain, J. D., and Bohannon, D.E. 1982. An in vivo hamster bioassay to assess the toxicity of particulates for the lungs. Toxicol. Appl. Pharmacol. 66: 9-29.

Becker, S., and Soukup, J. M. 1999. Exposure to urban air particulates alters the macrophage-mediated inflammatory response to respiratory viral infection. J. Toxicol. Environ. Health. Part A. 57: 445-457.

Becker, S., and Soukup, J. M. 2003. Coarse (PM(2.5-10)), fine (PM(2.5)), and ultrafine air pollution particles induce/increase immune costimulatory receptors on human bloodderived monocytes but $\mathrm{n}$ ot alveolar macrophages. J. Toxicol. Environ. Health. Part A. 66: 847-859.

Becker, S., Soukup, J. M., Gilmour, M. I., and Devlin, R. B. 1996. Stimulation of human and rat alveolar macrophages by urban air particulates: effects on oxidant radical generation and cytokine production. Toxicol. Appl. Pharmacol. 141: 637-648.

Becker, S. Fenton, M. J., and Soukup, J. M. 2002a. Involvement of microbial components and toll-like receptors 2 and 4 in cytokine responses to air pollution particles. Am. J. Respir. Cell Mol. Biol. 27: 611-618. 
Becker, S., Soukup, J. M., and Gallagher, J. E. 2002. Differential particulate air pollution induced oxidant stress in human granulocytes, monocytes, and alveolar macrophages. Toxicol. In Vitro. 16:209-218.

Bell, M. L., and Davis, D. L. 2001. Reassessment of the lethal London Fog of 1952: Novel indicators of acute and chronic consequences of acute exposure to air pollution. Environ. Health Perspect. 109 (suppl 3): 389-394.

Benko, V., Wagner, V., Wagnerova, M., and Reichrtova, E. 1983. Immunobiochemical findings in groups of individuals occupationally and non-occupationally exposed to emissions containing nickel and cobalt. J. Hyg. Epidemiol. Microbiol. Immunol. 27: 387394.

Benko, V., Wagner, V., Wagnerova, M., and Batora, J. 1988. Immunological profiles in workers of a power plant burning coal rich in arsenic content. J. Hyg. Epidemiol. Microbiol. Immunol. 32: 137-146.

Benson, J. M., Burt, D. G., and Carpenter, R. L. 1988. Comparative inhalation toxicity of nickel sulfate to $\mathrm{F} 344 / \mathrm{N}$ rats and B6C3F1 mice exposed for twelve days. Fund. Appl. Toxicol. 10: 164-178.

Benson, J. M., Chang, I., Cheng, Y., Hahn, F. F., Kennedy, C. H., Barr, E. B., Maples, K. R., and Snipes, M. B. 1995. Particle clearance and histopathology in lungs of F344/N rats and B6C3F1 mice inhaling nickel oxide or nickel sulfate. Fund. Appl. Toxicol. 28: 232244.

Berg, R. E., Cordes, C. J., and Forman, J. 2002. Contribution of CD8+ T cells to innate immunity: IFN- $\gamma$ secretion induced by IL-12 and IL-18. Eur. J. Immunol. 32: 2807-2816.

Berge, S. R., and Skyberg, K. 2003. Radiographic evidence of pulmonary fibrosis and possible etiologic factors at a nickel refinery in Norway. J. Environ. Monit. 5: 681-688.

Berry, J. P., Meignan, M., Escaig, F., and Galle, P. 1988. Inhaled soluble aerosols insolubilised by lysosomes of alveolar cells. Applicatio to some toxic compounds: Electron microprobe and ion microprobe studies. Toxicol. 52: 127-139.

Blanc, P., Wong, H., Bernstein, M. S., and Boushey, H. A. 1991. An experimental human model of metal fume fever. Ann. Internal Med. 114(11): 930-6.

Blesson, S., Thiery, J., Gaudin, C., Stancou, R., Kolb, J-P., Moreau, J-L., Theze, J., Mami-Chouaib, F., and Chouaib S. 2002. Analysis of the mechanism of human cytotoxic T lymphocyte response inhibition by NO. Internat. Immunol. 14: 1169-1178.

Bonner, J. C., Rice, A. B., Moonmaw, C. R., and Morgan, D. L. 2000. Airway fibrosis in rats induced by vanadium pentoxide. Am. J. Physiol. 278: L209-L216. 
Borja-Aburto, V. H., Castillejos, M., Gold, D. R., Bierzwinski, S., and Loomis, D. 2004. Mortality and ambient fine particles in southwest Mexico City, 1993-1995. Environ. Health Perspec. 106: 849-855.

Brewer, J. M., Conacher, M., Satoskar, A., Bluethmann, H., and Alexander, J. 1996. In interleukin-4-deficient mice, alum, not only generates $\mathrm{T}$ helper 1 responses equivalent to Freund's adjuvant, but continues to induce T helper-2 cytokine production. Eur. J. Immunol. 26: 2062-2066.

Brooks, S. M. 1981. Lung disorders resulting from the inhalation of metals. Clin. Chest Med. 2: 235-254.

Buchmeier, N. A., and Schreiber, R. D. 1985. Requirement of endogenous interferon- $\gamma$ production for the resolution of Listeria monocytogenes infection. Proc. Natl. Acad. Sci. USA. 82: 7404-7408.

Burleson, G. R., Keyes, L. L., and Stutzman, J. D. 1989. Immunosuppression of pulmonary natural killer cell activity by exposure to ozone. Immunopharmacol. Immunotoxicol. 11: 715-735.

Burnett, R. T., Brook, J., Dann, T., Delocla, C., Philips, O., Cakmak, S., Goldberg, M. S., and Krewski, D. 2000. Association between particulate- and gas-phase components of urban air pollution and daily mortality in eight Canadian cities. Inhal. Toxicol. 12(Suppl. 1): $15-39$.

Calderon-Garciduenas, L., Devlin, R. B., and Miller, F. J. 2000. Respiratory tract pathology and cytokine imbalance in clinically healthy children chronically and sequentially exposed to air pollutants. Med. Hypth. 55: 373-378.

Camner, P., Casarett-Bruce, M., Curdstedt, T., Jarstrand, C., Wiernik, A., Johansson, A., Lundborg, M., and Robertson, B. 1984. Toxicology of nickel. In: Nickel in the human environment, IARC Scientific Publication No. 53. Sunderman, F. W., Jr., ed. International Agency for Research on Cancer: Lyon, France. Pp. 267-276.

Campen, M. J., Nolan, J. P., Schladweiler, M. C. J., Kodavanti, U. P., Evansky, P. A., Costa, D. L., and Watkinson, W. P. 2001. Cardiovascular and thermoregulatory effects of inhaled PM-associated transition metals: a potential interaction between nickel and vanadium. Toxicol. Sci. 64: 243-252.

Campen, M. J., Nolan, J. P., Schladweiler, M. C. J., Kodavanti, U. P., Costa, D. L., and Watkinson, W. P. 2002. Cardiac and thermoregulatory effects of instilled particulate matter-associated transition metals in healthy and cardiopulmonary-compromised rats. $J$. Toxicol. Environ. Health, Part A. 65: 1615-1631. 
Carter, J. D., Ghio, A. J., Samet, J. M., and Devlin, R. B. 1997. Cytokine production by human epithelial cells after exposure to an air pollution particle is metal-dependent. Toxicol. Appl. Pharmacol. 146:180-188.

Castranova, V., Bowman, L., Miles, P. R., and Reasor, J. M. 1980a. Toxicity of metal ions to alveolar macrophages. Am. J. Ind. Med. 1: 349-357.

Castranova, V., Bowman, L., Reasor, J. M., and Miles, P. R. 1980b. Effects of heavy metal ions on selected oxidative metabolic processes in rat alveolar macrophages. Toxicol, Appl. Pharmacol. 53: 14-23.

Castranova, V., Bowman, L., Wright, J. R., Colby, H., and Miles, P. R. 1984. Toxicity of metallic ions in the lung: effects on alveolar macrophages and alveolar type II cells. $J$. Toxicol. Environ. Health. 13: 845-856.

Castranova, V., Vallyathan, V., Ramsey, D. M., McLaurin, J. L., Pack, D., Leonard, S., Barger, M. W., Ma, J. Y. C., Dalal, N. S., and Teass, A. 1997. Augmentation of pulmonary reactions to quartz inhalation by trace amount of iron-containing particles. Envrion. Health Perspect. 105 Suppl 5: 1319-1324.

Chen, C. Y., Wang, Y. F., Huang, W. R., and Huang, Y. T. 2003. Nickel induces oxidative stress and genotoxicity in human lymphocytes. Toxicol. Appl. Pharmacol. 189: 153-159.

Chen, B., Hong, C., Kan, H. 2004. Exposures and health outcomes from outdoor air pollutants in China. Toxicol. 198: 291-300.

Chien, P. S., Mak, O. T., and Huang, H. J. 2006. Induction of COX-2 protein expression by vanadate in A549 human lung carcinoma cell line through EGF receptor and p38 MAPK-mediated pathway. Biochem. Biophys. Res. Comm. 339: 562-568.

Chong, M. D., Shi, M. M., Love, J. A., Christiani, D. C., and Paulauskis, J. D. 2000. Regulation of chemokine mRNA expression in a rat model of vanadium-induced pulmonary inflammation. Inflammation. 24: 505-517.

Clarke, R. W., Catalano, P. J., Koutrakis, P., Murthy, G. G. K., Sioutas, C., Paulauskis, J., Coull, B., Ferguson, S., and Godleski, J. J. 1999. Urban air particulate inhalation alters pulmonary function and induces pulmonary inflammation in a rodent model of bronchitis. Inhal. Toxicol. 11: 637-656.

Cohen, M. D., Yang, Z., Qu, Q., Schlesinger, R. B., and Zeilikoff, J. T. 1996. Vanadium effects macrophage interferon- $\gamma$ binding and -inducible responses. Toxicol. Appl. Pharmacol. 138: 110-120. 
Cohen, M. D., Becker, S., Devlin, R., Schlesinger, R. B., and Zeilikoff, J. T. 1997. Effects of vanadium upon polyI:C-induced responses in rat lung and alveolar macrophages. J. Toxicol. Environ. Health. 51: 591-608.

Cohen, M. D., Sisco, M., Zelikoff, J. T., and Schlesinger, R. B. 2001. Ozone-induced modulation of cell-mediated immune responses in the lungs. Toxicol. Appl. Pharmacol. 171: 71-84.

Cohen, M. D., Sisco, M., Baker, K., Li, Y., Lawrence, D., van Loveren, H., Zelikoff, J. T., and Schlesinger, R. B. 2002. Effects of inhaled ozone on pulmonary immune cells critical to antibacterial responses in situ. Inhal. Toxicol. 14: 599-619.

Cohen, M. D. 2004. Pulmonary immunotoxicology of select metals: aluminum, arsenic, cadmium, chromium, copper, manganese, nickel, vanadium, and zinc. J. Immunotoxicol. 1: 39-69.

Condevaux, F., Guichard, J., Forichon, A., Aujoulat, M., and Descotes, J. 2001. Compared effects of morphine and nickel chloride on NK cell activity in vitro in rats and monkeys. J. Appl. Toxicol. 21: 431-434.

Conklin, A. W., Skinner, C. S., Felten, T. L., and Sanders, C. L. 1982. Clearance and distribution of intratracheally-instilled vanadium compounds in the rat. Toxicol. Lett. 11: 199-203.

Conner, M. W., Flood, W. H., Rogers, A. E., and Amdur, M. O. 1988. Lung injury in guinea pigs caused by multiple exposures to ultrafine zinc oxide: Changes in pulmonary lavage fluid. J. Toxicol. Environ. Health. 25: 57-69.

Costa, D. L., and Dreher, K. L. 1997. Bioavailable transition metals in particulate matter mediate cardiopulmonary injury in healthy and compromised animal models. Environ. Health Perspect. 105(Suppl. 5):1053-1060.

Dalrymple, S. A., Lucian, L. A., Slattery, R., McNeil, T., Aud, D. M., Fuchino, S., Lee, F., and Murray, R. 1995. Interleukin-6-deficient mice are highly susceptible to Listeria monocytogenes infection: correlation with inefficient neutrophilia. Infect. Immun.. 63: 2262-2268.

Daniels, M. J., Menache, M. G., Burleson, G. R., Graham, J. A., and Selgrade, M. J. 1987. Effects of $\mathrm{NiCl}_{2}$ and $\mathrm{CdCl}_{2}$ on susceptibility to murine cytomegalovirus and virusaugmented natural killer cell and interferon responses. Fund. Appl. Toxicol. 8: 443-453.

De Smedt, A. C., van den Heuvel, R. L., Zwi Berneman, N., and Schoeters, G. E. 2001. Modulation of phenotype, cytokine production, and stimulatory function of CD34+derived DC by $\mathrm{NiCl}_{2}$ and SDS. Toxciol. In Vitro. 15: 319-325. 
Di Gioacchino, M., Sabbioni, E., Di Giampaolo, L., Schiavone, C., Di Sciascio, M. B., Reale, M., Nicola, V., Qiao, N., Paganelli, R., Conti, P., and Boscolo, P. 2002. In vitro effects of vanadate on human immune functions. Ann. Clin. Lab. Sci. 32: 148-154.

Dockery, D. W., Pope III, C. A., Xu, X., Spengler, J. D., Ware, J. H., Fay, M. E., Ferris, B. G., and Speizer, F. E. 1993. An association between air pollution and mortality in six U.S. cities. N. Engl. J. Med. 329:1753-1759.

Dockery, D. W. and Pope III, C. A. 1994. Acute respiratory effects of particulate air pollution. Annu. Rev. Public Health. 15:107-132.

Dorger, M. and Krombach, F. 2002. Response of alveolar macrophages to inhaled particulates. Eur. Surg. Res. 34: 47-52.

Dormans, J. A., Steerenberg, P. A., Arts, J. H., van Bree, L., de Klerk, A., Verlaan, A. P. Bruijntjes, J. P., Beekhof, P., van Soolingen, D. and van Loveren, H. 1999. Pathological and immunological effects of respirable coal fly ash in male Wistar rats. Inhal. Toxicol. 11: 51-69.

Dreher, K. L., Jaskot, R. H., Lehmann, J. R., Richards, J. H., McGee, J. K., Ghio, A. J., and Costa, D. L. 1997. Soluble transition metals mediate residual oil fly ash-induced acute lung injury. J. Toxicol. Environ. Health. 50:285-305.

Drela, N., Zesko, I., and Biernat. P. 2002. Cytokines production is altered in mice exposed to airborne suspended matter. Immunopharm. Immunotox. 24: 497-525.

Drew, R. T., Gupta, B. N., Bend, J. R., and Hook, G. E. 1974. Inhalation studies with a glycol complex of aluminum-chloride-hydroxide. Arch. Environ. Health. 28: 321-6.

Drinker, P., Thompson, R. M., and Finn, J. L. 1927. Metal fume fever-IV. Threshold doses of zinc oxide, preventative measures, and the chronic effects of repeated exposures. J. Ind. Hyg. 9: 331-345.

Drummond, J. G., Aranyi, C., Schiff, L. J., Fenters, J. D., and Graham, J. A. 1986. Comparative study of various methods used for determining health effects of inhaled sulfates. Environ. Res. 41: 514-528.

Dunnick, J. K., Elwell, M. R., Benson, J. M., Hobbs, C. H., Hahn, F.F>, Haley, P. J., Chen, Y. S., and Edison, A. F. 1989. Lung toxicity after 13-week inhalation exposure to nickel oxide, nickel subsulfide, or nickel sulfate hexahydrate in $\mathrm{F} 344 / \mathrm{N}$ rats and $\mathrm{B} 6 \mathrm{C} 3 \mathrm{~F} 1$ mice. Fund. Appl. Toxicol. 12: 584-594.

Dutton, W. F. 1911. Vanadiumism. J. Am. Med. Assoc. 56: 1648-1658. 
Dye, J. A., Adler, K. B., Richards, J. H., and Dreher, K. L. 1997. Epithelial injury induced by exposure to residual oil fly ash particles: role of reactive oxygen species? Am. J. Respir. Cell Mol. Biol. 17: 625-633.

Dye, J. A., Adler, K. B., Richards, J. H., and Dreher, K. L. 1999. Role of soluble metals in oil fly ash-induced airway epithelial injury and cytokine gene expression. Am. J. Physiol. 277:L498-L510.

Dye, J. A., Lehmann, J. R., McGee, J. K., Winsett, D. W., Ledbetter, A. D., Everitt, J. I., Ghio, A. J, and Costa, D. L. 2001. Acute pulmonary toxicity of particulate matter filter extracts in rats: coherence with epidemiologic studies in Utah Valley residents. Environ. Health Perspect. 109(Suppl 3): 395-403.

Edel, J., and Sabbioni, E. 1988. Retention of intratracheally-instilled and ingested tetravalent and pentavalent vanadium in the rat. J. Trace Elem. Electrolytes Health Dis. 2: 23-30.

Ehring, G. R., Kerschbaum, H. H., Fanger, C. M., Eader, C., Rauer, H., and Calahan, M. D. 2000. Vanadate induces calcium signaling, $\mathrm{Ca} 2+$ release-activated $\mathrm{Ca} 2+$ channel activation, and gene expression in T lymphocytes and RBL-2H3 mast cells via thiol oxidation. J. Immunol. 164: 679-687.

Ehrlich, R., Findlay, J. C., and Gardner, D. E. 1978. Susceptibility to bacterial pneumonia of animals exposed to sulfates. Toxicol. Lett.1: 325-330.

Ehrlich, R. 1980. Interactions between environmental pollutants and respiratory infections. Environ. Health Perspect. 35: 89-100.

El-Mogazi, D., Lisk, D. J., Weinstein, L. H. 1988. A review of physical, chemical, and biological properties of fly ash and effects on agricultural ecosystems. Sci. Total Environ. 74: 1-37.

Emmerling, P., Finger, H., and Boekeml, J. 1975. Listeria monocytogenes infection in nude mice. Infect. Immun. 12: 437-439.

Englert, N. 2004. Fine particles and human health - a review of epidemiological studies. Toxicol. Let. 149:235-242.

Environmental Protection Agency. 1997. National Ambient Air Quality Standards for Particulate Matter; Final Rule. Fed. Regist. 62: 38651-38701.

Environmental Protection Agency. 2004. The Particle Pollution Report: Current understanding of air quality and emissions through 2003. pp 1-27.

Environmental Protection Agency. 2005. Air emissions trends - Continued progress through 2004. http://www.epa.gov/oar/airtrends/2005/econ-emissions.html 
Fernandez, A., Wendt, J. O L., Wolski, N., Hein, K. R. G., Wang, S., and Witten, M. L. 2003. Inhalation health effects of fine particles from co-combustion of coal and refusederived fuel. Chemosphere. 51: 1129-1137.

Ferris, B. G., Speizer, F. E., Spengler, J. D., Dockery, D. W., Bishop, Y. M. M., Wolfson, M., and Humble, C. 1979. Effects of sulfur oxides and respirable particulates on human health: methodology and demography of populations in study. Am. Rev. Respir. Dis. 120" 767-779.

Finehi, V. N., Que Hee, S. S., and Niemeier, R. W. 1981. Influence of exposure to aluminum chloride and fluoride dusts on some biochemical and physiological parameters in rats. In: Organ-directed toxicity chemical indices and mechanisms. Brown, S. S., Davies, D. S., eds. New York, NY: Pergamon Press, pp46-50.

Finkleman, R. B. 1995. Modes of occurance of environmentally-sensitive trace elements in coal. In: Environmental Aspects of Trace Elements. Swaine, D. J., and Goodarzi, F., eds. Kluwer Academic Publishers: Boston, MA, pp24-50.

Fisher, G. L., McNeill, K. L., and Democko, C. J. 1986. Trace element interactions affecting pulmonary macrophage cytotoxicity. Environ. Res. 39: 164-171.

Gao, F., Barchowsky, A., Nemec, A. A., and Fabisiak, J. P. 2004. Microbial stimulation by Mycoplasma fermentans synergistically amplifies IL-6 release by human lung fibroblasts in response to residual oil fly ash (ROFA) and nickel. Toxicol. Sci. 81: 467479.

Garcon, G., Gosset, P., Maunit, B., Zerimech, F., Cruesy, C., Muller, J. F., and Shirali, P. 2004. Influence of iron $(56 \mathrm{Fe} 2 \mathrm{O} 3$ or $54 \mathrm{Fe} 2 \mathrm{O} 3$ ) in the upregulation of cytochrome P450$1 \mathrm{~A} 1$ by benzo[a]pyrene in the respiratory tract of Sprague-Dawley rats. J. Appl. Toxicol. 24: 249-256.

Gardner, S. Y., Lehmann, J. R., and Costa, D. L. 2000. Oil fly ash-induced elevation of plasma fibrinogen levels in rats. Toxicol. Sci. 56: 175-180.

Gavett, S. H., Madison, S. L., Dreher, K. L., Winsett, D. W., McGee, J. K., and Costa, D. L. 1997. Metal and sulfate composition of residual oil fly ash determines airway hyperreactivity and lung injury in rats. Environ. Res. 72:162-172.

Gavett, S. H., Madison, S. L., Stevens, M. A., and Costa, D. L. 1999. Residual oil fly ash amplifies allergic cytokines, airway responsiveness, and inflammation in mice. Am. J. Respir. Crit. Care Med. 160: 1897-1904.

Ghio, A. J., Carter, J. D., Richards, J. H., Brighton, L. E., Lay, J. C., and Devlin, R. B. 1998. Disruption of normal iron homeostasis after bronchial instillation of an ironcontaining particle. Am. J. Physiol. 274: L396-L403. 
Ghio, A. J., Stonehuerner, J., Dailey, L. A., and Carter, J. D. 1999. Metals associated with both the water-soluble and insoluble fractions of an ambient air pollution particle catalyze an oxidative stress. Inhal. Toxicol. 11: 37-49.

Ghio, A. J., Kim, C., and Devlin, R. B. 2000. Concentrated ambient particles induce mild pulmonary inflammation in healthy volunteers. Am. J. Respir. Crit. Care Med. 162: 981998.

Ghio, A. J., Silbajoris, R., Carson, J. L., and Samet, J. M. 2002a. Biologic effects of oil fly ash. Environ. Health Perspect. 110(Suppl. 1):89-94.

Ghio, A. J., Suliman, H. B., Carter, J. D., Abushamaa, A. M., and Folz, R. J. 2002 b. Overexpression of extracellular superoxide dismutase decreases lung injury after exposure to oil fly ash. Am. J. Phys. 283: L211-L218.

Ghio, A. J. 2004. Biological effects of Utah Valley ambient air particles in humans: a review. J. Aerosol Med. 17: 157-164.

Gibb, F. R., and Morrow, P. E. 1962. Alveolar clearance in dogs after inhalation of an iron $^{59}$ oxide aerosol. J. Appl. Physiol. 17: 429-432.

Gilmour, M. I., Daniels, M., McCrillis, R. C., Winsett, D., and Selgrade, M. K. 2001. Air pollution-enhanced respiratory disease in experimental animals. Environ. Health Perspect. 109: 619-622.

Gilmour, M. I., Schladwieler, M. C., Richards, J. H., Ledbetter, A. D., and Kodavanti U. P. 2004. Hypertensive rats are susceptible to TLR4-mediated signaling following exposure to combustion source particulate matter. Inhal. Toxicol. 16 suppl 1: 5-18.

Goldsmith, C. A., Frevert, C., Imrich, A., Siuotas, C., and Kobzik, L. 1997. Alveolar macrophage interaction with air pollution particulates. Environ. Health Perspect. 105 Suppl 5: 1191-1195.

Goldsmith, C. A., Imrich, A., Danaee, H., Ning, Y., and Kobzik, L. 1998. Analysis of air pollution particulate-mediated oxidant stress in alveolar macrophages. J. Toxicol.

Environ. Health. Part A. 54: 529-545.

Goldsmith, C. A., Hamada, K., Ning, Y. Y., Qin, P., Murthy, G. G. K., Lawrence, J. and Kobzik, L. 1999. Effects of environmental aerosols on airway hyperresponsiveness in a murine model of asthma. Inhal. Toxicol. 11:981-998.

Goldstein, E., Lippert, W., Chang, D. P., and Tarkington, B. 1979. Effect of near ambient exposures to sulfur dioxide and ferrous sulfate particles on murine pulmonary defense mechanisms. Arch. Environ. Health. 34: 424-431. 
Gordon, T., Chen, L. C., Fine, J. M., Schlesinger, R. B., Su, W., Kimmel, T. A., and Amdur, M. O. 1992. Pulmonary effects of inhaled zinc oxide in human subjects, guinea pigs, rats, and rabbits. Am. Ind. Hyg. Assoc. J. 53: 503-509.

Goutet, M., Ban, M., and Binet, S. 2000. Effects of nickel sulfate on pulmonary natural immunity in Wistar rats. Toxicol. 145: 15-26.

Graham, J. A., Gardner, D. E., Waters, M. D., and Coffin, D.C. 1975. Effect of trace metals on phagocytosis by alveolar macrophages. Infect. Immun. 11: 1278-1283.

Hadnagy, W., and Seemayer, N. H. 1994. Inhibition of phagocytosis of human macrophages induced by airborne particulates. Toxicol. Lett. 72: 23-31.

Hahon, N., Booth, J. A., Green, F., and Lewis, T. R. 1985. Influenza virus infection in mice after exposure to coal dust and diesel engine emissions. Environ. Res. 37: 44-60.

Haley, P. J., Shopp, G. M., Benson, J. M., Cheng, Y. S., Bice, D. E., Luster, M. I., Dunnick, J. K., and Hobbs, C. H. 1990. The immuntoxicity of three nickel compounds following 13-week inhalation exposure in the mouse. Fund. Appl. Toxicol. 15: 476-487.

Hamada, K., Goldsmith, C. A., Suzaki, Y., Goldman, A., and Kobzik, L. 2002. Airway hyperresponsiveness caused by aerosol exposure to residual oil fly ash leachate in mice. J. Toxicol. Environ. Health, Part A. 65: 1351-1365.

Hamilton, R. F., Holian, A., and Morandi, M. T. 2004. A comparison of asbestos and urban particulate matter in the in vitro modifcation of human alveolar macrophage antigen-presenting cell function. Exp. Lung Res. 30: 147-162.

Hardy, J. A., and Aust, A. E. 1995. Iron in asbestos chemistry and carcinogenicity. Chem. Rev. 95: 97-118.

Harkin, A., Hynes, M. J., Masterson, E., Kelly, J. P., O’Donnell., J. M., and Conner, T. J. 2003. A toxicokinetic study of nickel-induced immunosuppression in rats.

Immunopharmacol. Immunotoxicol. 25: 655-670.

Harrod, K. S., Jaramillo, R. J., Rosenbberger, C. L., Wang, S. Z., Berger, J. A., McDonald, J. D., and Reed, M. D. 2003. Increased susceptibility to RSV infection by exposure to inhaled diesel engine emissions. Am. J. Respir. Cell Mol. Biol. 28: 451-463.

Harrod, K. S., Jaramillo, R. J., Berger, J. A., Gigliotti, A. P., Seilkop, S. K., and Reed, M. D. 2005. Inhaled diesel engine emissions reduce bacterial clearance and exacerbate lung disease to Pseudomonas aeruginosa infection in vivo. Toxicol. Sci. 83: 155-165.

Hatch, G. E., Slade, R., Boykin, E., Hu, P. C., Miller, F. J., and Gardner, D. E. 1981. Correlation of effects of inhaled versus intratracheally-injected metals on susceptibility to respiratory infection in mice. Am. Rev. Respir. Dis. 124: 167-173. 
Hatch, G. E., Boykin, E., Graham, J. A., Lewtas, J., Pott, F., Loud, K., and Mumfor, J. L. 1985. Inhalable particles and pulmonary host defense: In vivo and in vitro effects of ambient air and combustion particles. Environ. Res. 36:67-80.

Hauser, R., Elreedy, S., Hoppin, J. A., and Christiani, D. C. 1995a. Airway obstruction in boilermakers exposed to fuel oil ash. Am. J. Respir. Crit. Care Med. 152: 1478-1484.

Hauser, R., Elreedy, S., Hoppin, J. A., and Christiani, D. C. 1995b. Upper airway response in workers exposed to fuel oil ash: nasal lavage analysis. Occup. Environ. Med. 52: $353-358$.

Hauser, R., Eisen, E. A., Pothier, L., Lewis, D., Bledsoe, T., and Christiani, D. C. Spirometric abnormalities associated with chronic bronchitis, asthma, and airway hyperresponsiveness among boilermaker construction workers. Chest. 121: 2052-2060.

Havell, E. A., Moldawer, L. L., Helfgott, D., Kilian, P. L., and Sehgal, P. B. 1992.Type I IL-1 receptor blockade exacerbates murine listeriosis. J. Immunol. 148: 1486-1492.

Heinrich, J., Hoelscher, B., Wjst, M., Ritz, B., Cyrys, J., and Wichmann, H. 1999. Respiratory diseases and allergies in two polluted areas in East Germany. Environ. Health Perspect. 107: 53-62.

Hertz-Picciotto, I., Herr, C. E., Yap, P. S., Dostal, M., Shumway, R. H. Ashwood, P., Lipsett, M., Joad, J. P., Pinkerton, K. E., and Sram, R. J. 2005. Air pollution and lymphocyte phenotype proportions in cord blood. Environ. Health Perspect. 113: 13911398.

Hippeli, S. C., and Elstner, E. F. 1990. Influence of diesel soot particles and sulfite on functions of polymorphonuclear leukocytes. Free Radic. Res. Comm. 11: 29-38.

Hirano, S., Asami, T., Kodama, N., and Suzuki, K. T. 1994. Correlation between inflammatory cellular responses and chemotactic activity in bronchoalveolar lavage fluid following intratracheal instillation of nickel sulfate in rats. Arch. Toxicol. 68: 444-449.

Hitzfeld, B., Friedrichs, K. H., Ring, J., and Behrendt, H. 1997. Airborne particulate matter modulates the production of reactive oxygen species in human polymorphonuclear granulocytes. Toxicol. 120: 185-195.

Hoffman, R. A., Mahidhara, R. S., Wolf-Johnston, A. S., Lu, L., Thomsaon, A. W., and Simmons, R. L. 2002. Differential modulation of CD4 and CD8 T cell proliferation by induction of nitric oxide synthase in antigen presenting cells. Transplantation. 74:836845. 
Holgate, S. T., Devlin, R. B., Wilson, S. J., and Frew, A. J. 2003. Health effects of acute exposure to air pollution. Part II: Healthy subjects exposed to concentrated ambient particles. Res. Rep. Health Eff.

Inst. 112: 31-71.

Hollingsworth, J. W. II, Cook, D. N., Brass, D. M., Walker, J. K. L., Morgan, D. L., Foster, W. M., and Schwartz, D. A. 2004. The role of toll-like receptor 4 in environmental airway injury in mice. Am. J. Respir. Crit. Care Med. 170: 126-132.

Hopkins, L. L., and Tilton, B. E. 1966. Metabolism of trace amounts of vanadium ${ }^{48}$ in rat organs and liver subcellular particles. Am. J. Physiol. 211: 169-172.

Hovey, H. S., Habib, M., and Wells, I. D. 1983. Asthma and IgE antibodies induced by chromium and nickel salts. J. All. Clin. Immunol. 72: 407-412.

Hsieh, C. S., Macatonia, S. E., Tripp, C. S., Wolf, S. F., O'Garra, A., and Murphy, K. M. 1993. Development of $\mathrm{T}_{\mathrm{H}} 1 \mathrm{CD} 4+\mathrm{T}$ cells through IL-12 produced by Listeria-induced macrophages. Science. 260: 547-9.

Huang, Y. C., Wu, W., Ghio, A. J., Carter, J. D., Silbajoris, R., Devlin, R. B., and Samet, J. M. 2002a. Activation of EGF receptors mediates pulmonary vasoconstriction induced by residual oil fly ash. Exp. Lung Res. 28: 19-39.

Huang, C., Li, J., Zhang, Q., and Huang, X. 2002b. Role of bioavailable iron in coal dustinduced activation of activator protein-1 and nuclear factor of activated T cells. Am. J. Respir. Cell Mol. Biol. 27: 568-574.

Huang, Y. C., Soukup, J., Harder, S. and Becker, S. 2003a. Mitochondrial oxidant production by a pollutant dust and NO-mediated apoptosis in human alveolar macrophage. Am J. Physiol. 284:C24-C32.

Huang, Y. C., Ghio, A. J., Stonehuerner, J., McGee, J. Carter, J. D., Grambow, S. C., and Devlin, R. B. 2003b. The role soluble components in ambient fine fine particle-induced changes in human lungs and blood. Inhal. Toxicol. 15: 327-342.

Huang, X., Li, W., Attfield, M. D., Nadas, A., Frenkel, K., and Finkelman, R. B. 2005. Mapping and prediction of coal workers' pneumoconiosis with bioavailable iron content in the bituminous coals. Environ. Health Perspect. 113: 964-968.

Hunt, A., Abraham, J. L., Judson, B., and Berry, C. L. 2003. Toxicologic and epidemiologic clues from the characterization of the 1952 London smog fine particulate matter in archival autopsy lung tissues. Environ. Health Perpsect. 111: 1209-1214. 
Hunter, C. A., Chizzonite, R., and Remington, J. S. 1995. IL-1 beta is required for IL-12 to induce production of IFN-gamma by NK cells. A role for IL-1 beta in the T cellindependent mechanism of resistance against intracellular pathogens. J. Immunol. 155: 4347-4354.

Irsigler, G. B., Visseer, P. J., and Spangenberg, P. 1999. Asthma and chemical bronchitis in vanadium plant workers. Am. J. Ind. Med. 35: 336-374.

Jacob, D. J., and Gottlieb, E. W. 1989. Chemistry of a polluted cloudy boundry layer. J. Geophys. Res. 94: 12957-13002.

Jakab, G. J. 1993. The toxicologic interactions resulting from inhalation of carbon black and acrolein on pulmonary antibacterial and antiviral defenses. Toxicol. Appl. Pharmacol. 121: 167-175.

Janeway, C. A., Traverds, P., Walport, M., and Schlomchik (eds). 2001. Immunobiology. $5^{\text {th }}$ Ed. Garland Publishing, New York, NY.

Jaspers, I., Ciencewicki, J. M., Zhang, W., Brighton, L. E., Carson, J. L., Beck, M. A., and Madden, M. C. 2005. Diesel exhaust enhances influenza virus infections in respiratory epithelial cells. Toxicol. Sci. 85: 990-1002.

Jiang, N., Dreher, K. L., Dye, J. A., Li, Y., Richards, J. H., Martin, L. D., and Adler, K. B. 2000. Residual oil fly ash induces cytotoxicity and mucin secretion by guinea pig tracheal epithelial cells via an oxidant-mediated mechanism. Toxicol. Appl. Pharmacol. 163:221-230.

Johnson, F. A., and Stonehill, R. B., Chemical pneumonitis from inhalation of zinc chloride. Dis. Chest. 40: 619-624.

Johansson, A., and Camner, P. 1980. Effects of nickel dust on rabbit alveolar epithelium. Environ. Res. 22: 510-516.

Johansson, A., Curstedt, T., Rasool, O., Jarstrand, C., and Camner, P. 1992. Macrophage reaction in rabbit lung following inhalation of iron chloride. Environ. Res. 58: 66-79.

Kacew, S., Parulekar, M. R., and Merali, Z. 1982. Effects of parenteral vanadium administration on pulmonary metabolism of rats. Toxicol. Lett. 11: 119-124.

Kadiiska, M. B., Mason, R. P., Dreher, K. L., Costa, D. L., and Ghio, A. J. 1997. In vivo evidence of free radical formation in the rat lung after exposure to an emission source air pollution particle. Chem. Res. Toxicol. 10: 1104-1108.

Kasprzak, K. S., Sunderman, F. W. Jr., and Salnikow, K. 2003. Nickel carcinogenesis. Mutation Res. 533: 67-97. 
Kelleher, P., Pacheco, K., and Newman, L. S. 2000. Inorganic dust pneumonias: the metal-related parenchymal disorders. Environ. Health Perspect. 108 Supple 4: 685-696.

Kim, K., Lee, S. H., Seo, Y. R., Perkins, S. N., and Kasprzak, K. S. 2002. Nickel(II)induced apoptosis in murine $\mathrm{T}$ cell hybridoma cells is associated with increased fas ligand expression. Toxicol. Appl. Pharmacol. 185: 41-47.

Kim, J. E., Hauser, R., Wand, M. P., Herrick, R. F., Amarasiriwardena, C. J., and Christiani, D. C. 2003. The association of expired nitric oxide with occupational particulate metal exposure. Environ. Res. 93: 158-166.

Kim, J. E., Mukherjee, S., Ngo, L., and Christiani, D. C. 2004. Urinary 8-hydroxy-2'deoxyguanasine as a biomarker of oxidative DNA damage in workers exposed to fine particulates. Environ. Health Perspect. 112: 666-671.

Kivuoloto, M., Rasanen, O., Rinne, A., and Rissanen, M. 1979. Effects of vanadium on the upper respiratory tract of workers in a vanadium factory. A macroscopic and microscopic study. Scand. J. Work Environ. Health. 5: 50-58.

Kleinerman, J. 1974. Industrial pulmonary diseases: Coal workers' pneumoconiosis, berylliosis, and miscellaneous causes. In: Textbook of Pulmonary Diseases. Bau, G. L. ed. Little, Brown: Boston, MA. Pp 509-524.

Kleinfeld, M., Messite, J., Looyman, O., and Shapiro, J. 1969. Welders' siderosis: a clinical roentgenographic and physiological study. Arch. Environ. Health. 19: 70-73.

Kleinman, M. T., Sioutas, C., Chang, M. C., Boere, A. J. F., and Cassee, F. R. 2003. Ambient fine and coarse particle suppression of alveolar macrophage functions. Toxicol. Lett. 137: 151-158.

Kleinsasser, N., Dirschedl, P., Staudenmaier, R., Harreus, U., and Wallner, B. 2003. Genotoxic effects of vanadium pentoxide on human peripheral lymphocytes and mucosal cells of the upper aerodigestive tract. Internat. J. Environ. Health Res. 13: 373-379.

Knight, J. A., Plowman, M. R., Hopfer, S. M., and Sunderman, F. W. Jr. 1991.

Pathological reactions in lung, liver, thymus, and spleen after subacute parenteral administration of nickel sulfate. Ann. Clin. Lab. Sci. 21: 275-283.

Kodavanti, U. P. Jaskot, R. H., Costa, D. L., and Dreher, K. L. 1997. Pulmonary proinflammatory gene induction following acute exposure to residual oil fly ash: roles of particle-associated metals. Inhal. Toxicol. 9: 679-701.

Kodavanti, U. P., Hauser, R., Christiani, D. C., Meng, Z. H., McGee, J., Ledbetter, A., Richards, J., and Costa, D. L. 1998. Pulmonary responses to oil fly ash particles in the rat differ by virtue of their specific soluble metals. Toxicol. Sci. 43:204-212. 
Kodavanti, U. P., Mebane, R., Ledbetter, A., Krantz, T., McGee, J., Jackson, M. C., Walsh, L., Hilliard, H., Chen, B. Y., Richards, J., and Costa, D. L. 2000. Variable pulmonary responses from exposure to concentrated ambient air particles in a rat model of bronchitits. Toxicol. Sci. 54: 441-451.

Kodavanti, U. P., Schladweiler, M. C. J., Richards, J. R., and Costa, D. L. 2001. Acute lung injury from intratracheal exposure to fugitive residual oil fly ash and its constituent metals in normo- and spontaneously hypertensive rats. Inhal. Toxicol. 13:37-54.

Kodavanti, U. P., Schladweiler, M. C. J., Ledbetter, A.,Hauser, R., Christiani, D. C., Samet, J. M., McGee, J., Richards, J. R., and Costa, D. L. 2002. Pulmonary and systemic effects of zinc-containing emission particles in three rat strains: multiple exposure scenarios. Toxicol. Sci. 70: 73-85.

Kopf, M., Baumann, H., Freer, G., Freudenberg, M., Lamers, M., Kishimoto, T., Zinkernagel, R., Bluethmann, H., and Kohler, G. 1994. Impaired immune and acutephase responses in interleukin-6-deficient mice. Nature. 368: 339-342.

Krishna, M. T., Chauhan, A. J., Frew, A. J., and Holgate, S. T. 1998. Toxicological mechanisms underlying oxidant pollutant-induced airways injury. Rev. Environ. Health. 13: 59-71.

Kuschner, W. G., D’Alessandro, A., Wintermeyer, S. F., Wong, H., Boushey, H. A., and Blanc, P. D. 1997. Early pulmonary cytokine responses to zinc oxide fume inhalation. Environ. Res. 75: 7-11.

Ladel, C. H., Flesch, I. E., Arnoldi, J., and Kaufmann, S. H. 1994. Studies with MHCdeficient knock-out mice reveal impact of both MHC I- and MHC II-dependent T cell responses on Listeria monocytogenes infection. J. Immunol. 153: 3116-3122.

Laden, F., Neas, L. M., Dockery, D. W., and Schwartz, J. 2000. Association of fine particulate matter from different sources with daily mortality in six U. S. cities. Environ. Health. Perspect. 108: 941-947.

Lambert, A. L., Dong, W., Selgrade, M. K., and Gilmour, M. I. 2000. Enhanced allergic sensitization by residual oil fly ash particles is mediated by soluble metal constituents. Toxicol. Appl. Pharmacol.165: 84-93.

Lay, J. C., Bennett, W. D., Ghio, A. J., Bromberg, P. A., Costa, D. L., Kim, C. S., Koren, and Devlin, R. B. 1999. Cellular and biochemical response of the human lung after intrapulmonary instillation of ferric oxide particles. Am. J. Respir. Cell Mol. Biol. 20: 631-642.

Lay, J. C., Zeman, K. L., Ghio, A. J., and Bennett, W. D. 2001. Effects of inhaled iron oxide particles on alveolar epithelial permeability in normal subjects. Inhal. Toxicol. 13: 1065-1078. 
Lehman, K. B. 1910. Study of technically and hygienically important gases and steams. XIV. Foundry or zinc fever. Arch. Hyg. 72: 358-381.

Leonardi, G. S., Houthuijs, D., Steerenberg, P. A., Fletcher, T., Armstrong, B., and Antova, T. 2000. Immune biomarkers in relation to exposure to particulate matter: a cross-sectional survey in 17 cities in central Europe. Inhal. Toxicol.12(Suppl 4): 1-14.

Lee, R. E., Goranson, S. S., Enrione, R. E., and Morgan, G. B. 1972. National air surveillance cascade impactor network. II. Size distribution measurements or ftrace element components. Environ. Sci. Technol. 6: 1025-1030.

Lees, R. E. 1980. Changes in lung function after exposure to vanadium compounds in fuel oil ash. Br. J. Ind. Med. 37: 253-256.

Lehnert, B. E., and Morrow, P. E. 1985. Characteristics of alveolar macrophages following the deposition of a low burden of iron oxide in the lung. J. Toxicol. Environ. Health. 16: 855-868.

Levy, B. S., Hoffman, L., and Gottsegen, S. 1984. Boilermakers' bronchitis: respiratory tract irritation associated with vanadaium pentoxide exposure during oil-to-coal conversion of a powerp lant. J. Occup. Med. 26:567-570.

Lewis, A. B., Taylor, M. D., Roberts, J. R., Leonard, S. S., Shi, X., and Antonini, J. M. 2003. Role of metal-induced reactive oxygen species generation in lung responses caused by residual oil fly ash. J. Biosci. 28:13-18.

Lindblad, EB. 2004. Aluminum compounds for use in vaccines. Immunol. Cell Biol. 82:497-505.

Lindroos, P. M., Coin, P. G., Badgett, A., Morgan, D. L., and Bonner, J. C. 1997. Alvoelar macrophages stimulated with titanium dioxide, chrysotile asestos, and residual oil fly ash upregulate the PDGF receptor- $\alpha$ on lung fibroblasts through an IL-1 $\beta$ dependent mechanism. Am. J. Respir. Cell Mol. Biol. 16: 283-292.

Lindenschmidt, R. C., Driscoll, K. E., Perkins, M. A., Higgins, J. M., Maurer, J. K., and Belfiore, K. A., 1990. The comparison of a fibrogenic and two nonfibrogenic dusts by bronchoalveolar lavage. Toxicol. Appl. Pharmacol. 102: 268-281.

Lucesoli, F., Caligiuri, M., Roberti, M. F., Perazzo, J. C., and Fraga, C. G. 1999. Dosedependent increase in oxidative damage in the testes of rats subjected to acute iron overload. Arch. Biochem. Biophys. 372: 37-43.

MacLean, J. A., Xia, W., Pinto, C. E., Zhao, L., Liu, H. W., and Kradin, R. L. 1996. Sequestration of inhaled particulate antigens by lung phagocytes. A mechanism for the effective inhibition of pulmonary cell-mediated immunity. Am. J. Pathol. 148: 657-666. 
MacMicking, J. D., Nathan, C., Hom, G., Chartrain, N., Fletcher, D. S., Trumbauer, M., Stevens, K., Xie, Q. W., Sokol, K., Hutchinson, N., Chen, H. and Mudget, J. S. 1995. Altered responses to bacterial infection and endotoxic shock in mice lacking inducible nitric oxide synthase. Cell. 81: 641-650.

Marrs, T. C., Colgrave, H. F., Edington, J. A. G., Brown, R. F., Cross, N. L. 1988. The repeated dose toxicity of a zinc oxide/hexachloroethane smoke. Arch. Toxicol. 62(2-3): 123-32.

McGregor, D. D., Koster, F. T., and Mackaness, G. B. 1970. The short-lived small lymphocyte as a mediator of cellular immunity. Nature. 228: 855-856.

Medeiros, N., Jr., Rivero, D. H. R. F., Kasahara, D. I., Saiki, M., Godleski, J. J., Koutrakis, P., Capelozzi, V. L., Saldiva, P. H. N., and Antonangelo, L. 2004. Acute pulmonary and hematological effects of two types of particle surrogates are influenced by their elemental composition. Environ. Res. 95: 62-70.

Meyers, R. A. 1982.Coal Structure. Academic Press: New York, New York.

Miller, J. C., Morton, J. J. P., and Soileua, S. D. 2001. Chapter 14: Metals. In: Principles and Methods of Toxicology, $4^{\text {th }}$ Edition. Hayes, A. W., ed. Taylor and Francis:

Philadelphia, PA.

Ministry of Health. 1954. Mortality and Morbidity during theLondon Fog of December 1952. Reports on Public Health and Medical Subjects No. 95. London: London Ministry of Health.

Molinelli, A. R., Madden, M. C., McGee, J. K., Stonehuerner, J. G., and Ghio, A. J. 2002. Effect of metal removal on the toxicity of airborne particulate matter from the Utah Valley. Inhal. Toxicol. 14: 1069-1086.

Morrow, P. E., Gibb, F. R., and Johnson, L. 1964. Clearance of insoluble dust from the lower respiratory tract. Healht Phys. 10: 543-555.

Mosmann, T. R., and Sad, S. 1996. The expanding universe of T-cell subsets: Th1, Th2, and more. Immunol. Today. 17:138-146.

Mukherjee, S. Palmer, L. J., Kim, J. Y., Aeschliman, D. B., Houk, R. S., Woodin, M. A., and Christiani, D. C. 2004. Smoking status and occupational exposure affects oxidative DNA injury in boilermakers exposed to metal fume and residual oil fly ash. Cancer Epidemiol Biomarkers Prev. 13: 432-438.

Munger, J. W., Jacob, D. J., Waldman, J. M., and Hoffmann, M. R. 1983. Fogwater chemistry in an urban atmosphere. J. Geophys. Res. 88: 5109-5121. 
Musk, A. W., and Tees, J. G. 1982. Asthma caused by occupational exposure to vanadium compounds. Med. J. Aust. 1: 183-184.

Muzyka, V., Bogovski, S., Scheepers, P., Volf, J., and Kusova, J. 2003. Effects of occupational exposure to diesel exhaust on porphyrin metabolism in lymphocytes of workers employed at black coal and oil-shale mines. Am. J. Ind. Med. 44: 70-74.

Natusch, D. F. S., Wallace, J. R., and Evans, C. A., Jr. 1974. Toxic trace elements: preferential concentration in respirable particles. Science. 183: 202-204.

Nickol, A. D., and Bonventre, P. F. 1977. Anomolous high native resistance of athymic mice to bacterial pathogens. Infect. Immun. 18: 636-645.

NIOSH. 2005. NIOSH pocket guide to chemical hazards: Iron salts (soluble, as Fe). NIOSH Publication No. 2005-151. National Institute for Occupational Safety and Health. Available: www.cdc.gov/niosh/ngp/npgd0346.html.

Neighbors, M., Xu, X., Barrat, F. J., Ruuls, S. R., Churakova, T. Debets, R., Bazan, J. F., Kastelein, R. A., Abrams, J. S., and O'Garra, A. 2001. A critical role for interleukin 18 in primary and memory effector responses to Listeria monocytogenes that extends beyond its effects on interferon $\gamma$ production. J. Exp. Med. 194: 343-354.

Nemery, B. 1990. Metal toxicity and the respiratory tract. Eur. Respir. J. 3: 202-219.

Nadadur, S. S., Schladweiler, M. C., and Kodavanti, U. P. 2000. A pulmonary rat gene array for screening altered expression profiles in air pollutant-induced lung injury. Inhal. Toxicol. 12:1239-1254.

Nadadur, S. S., and Kodavanti, U. P. 2002. Altered gene expression profiles of rat lung in response to an emission particulate and its metal constituents. J. Toxicol. Environ. Health. 65:1333-1350.

Nobutomo, K. 1978. Air pollution and cytological changes in sputum. Lancet. 1(8063): 523-526.

NTP. 1996a. NTP technical report on the toxicology and carcinogenesis studies of nickel oxide (CAS No. 1313-99-1) in F344/N rats and B6C3F1 mice (inhalation studies). NTPTRS No. 451. Research Triangle Park, NC: U. S. Department of Health and Human Services, Public Health Service, National Institute of Health.

NTP. 1996b. NTP technical report on the toxicology and carcinogenesis studies of nickel subsulfide (CAS No. 12035-72-2) in F344/N rats and B6C3F1 mice (inhalation studies). NTP-TRS No. 453. Research Triangle Park, NC: U. S. Department of Health and Human Services, Public Health Service, National Institute of Health. 
NTP. 1996c. NTP technical report on the toxicology and carcinogenesis studies of nickel sulfate hexahydrate (CAS No. 10101-97-0) in F344/N rats and B6C3F1 mice (inhalation studies). NTP-TRS No. 454. Research Triangle Park, NC: U. S. Department of Health and Human Services, Public Health Service, National Institute of Health.

NTP (National Toxicology Program). 2002. NTP toxicology and carcinogenesis studies of vanadium pentoxide (CAS No. 1314-62-1) in F344/N rats and B6C3F1 mice (inhalation). Nat. Toxciol. Prog. Tech. Report Series. 507: 1-343.

Nurkiewicz, T. R., Porter, D. W., Barger, M., Castranova, V., Boegehold, M. A. 2004. Particulate matter exposure impairs systemic microvascular endothelium-dependent dilation. Environ. Health Perspect. 112: 1299-1306.

Ohanti, T., Nakagawa, S., Kurosawa, M., Mizuashi, M., Ozawa, M., and Aiba, S. 2005. Cellular basis of the role of diesel exhaust particles in inducing Th2-dominant response. J. Immunol. 174: 2412-2419.

Olakanmi, O., McGowen, S. E., Hayek, M. B., and Britigan, B. E. 1993. Iron sequestration by macrophages decreases the potential for extracellular hydroxyl radical formation. J. Clin. Invest. 91: 889-899.

Oller, A. R. Respiratory carcinogenicity assessment of soluble nickel compounds. Environ. Health Perspect. 110 Suppl 5: 851-848.

Oller, A. R., Costa, M., and Oberdorster, G. 1997. Carcinogenicity assessment of selected nickel compounds. Toxicol. Appl. Pharmacol. 143: 152-166.

Omara, F. O., Brousseau, P., Blakely, B. R., and Fournier, M. 1998. Iron, zinc, and copper. In: Immunotoxicology of environmental and Occupational Metals. Zelikoff, J. T., and Thomas, P. T., eds. Taylor and Francis, London, England. pp.163-194.

Omara, F. O., Fournier, M., Vincent, R., and Blakley, B. R. 2000. Suppression of rat and mouse lymphocyte function by urban air particulates (Ottawa Dust) is reversed by $\mathrm{N}$ acetylcysteine. J. Toxicol. Environ. Health. Part A. 59: 67-85.

Pamer, E. G. 2004. Immune responses to Listeria monocytogenes. Nature Rev. 4:812823.

Pinkerton, K. E., Zhou, Y-M., Teague, S. V., Peake, J. L., Walther, R. C., Kennedy, I. M., Leppert, V. J., and Aust, A. E. 2004. Reduced lung cell proliferation following shortterm exposure to ultrafine soot and iron particles in neonatal rats: key to impaired lung growth? Inhal. Toxicol. 16 Suppl 1: 73-81.

Pope III, C. A., Dockery, D. W., and Schwartz, J. 1995. Review of epidemiological evidence of health effects of particulate air pollution. Inhal. Toxicol. 7:1-18. 
Pope III, C. A., Burnett, R. T., Thurston, G. D., Thun, M. J., Calle, E. E., Krewski, D., and Godleski, J. J. 2004. Cardiovascular mortality and long-term exposure to particulate air pollution: epidemiological evidence of general pathophysiological pathways of disease. Circ. 109: 71-77.

Port, C. D., Fenters, J. D., Ehrlich, R., Coffin, D. L., and Gardner, D. 1975. Interaction of nickel oxide and influenza infection in hamsters. Environ. Health Perspect. 10: 268.

Portney, D. A., Jacks, P. S., and Hinrichs, D. J. 1988. Role of hemolysin for the intracellular growth of Listeria monocytogenes. J. Exp. Med. 167: 1459-1471.

Prahalad, A. K., Inmon, J., Dailey, L. A., Madden, M. C., Ghio, A. J., and Gallagher, J. E. 2001. Air pollution particles mediated oxidative DNA base damage in a cell free system and in human airway epithelial cells in relation to particulate metal content and bioreactivity. Chem. Res. Toxicol. 14: 879-887.

Prieditis, H., and Adamson, I. Y. R. 2002. Comparative pulmonary toxicity of various soluble metals found in urban particulate dusts. Exp. Lung Res. 28: 563-576.

Reasor, M. J., McCloud, C. M., DiMatteo, M., Schafer, R., Ima, A., and Lemaire, I. 1996. Effects of amiodarone-induced phospholipidosis on pulmonary host defense functions in rats. Proc. Soc. Expt. Biol. Med. 211: 346-352.

Ress, N. B., Chou, B. J., Renne, R. A., Dill, J. A., Miller, R. A., Roycroft, J. H., Hailey, J. R., Haseman, J. K., and Bucher, J. R. 2003. Carcinogenicity of inhaled vanadium pentoxide in F344/N rats and B6C3F1 mice. Toxicol. Sci. 74: 287-296.

Riley, M. R., Boesewetter, D. E., Turner, R. A., Kim, A. M., Clloier, J. M., and Hamilton, A. 2005. Comparison of the sensitivity of three lung derived cell lines to metals from combustion derived particulate matter. Toxicol. In Vitro. 19: 411-419.

Roberts, E. S., Richards, J. H., Jaskot, R., and Dreher, K. L. 2003. Oxidative stress mediates air pollution particle-induced acute lung injury and molecular pathology. Inhal. Toxicol. 15: 1327-1346.

Roberts, E., Charbonaeu, L., Espina, V., Liotta, L., Petricoin, E., and Dreher, K. 2004. Application of laser capture microdissection and protein microarray technologies in the molecular analysis of airway injury following pollution particle exposure. J. Toxicol. Environ. Health. Part A. 67: 851-861.

Rogers, H. W., Tripp, C. S., Schreiber, R. D., and Unanue, E. R.1994 Endogenous IL-1 is required for neutrophil recruitment and macrophage activation during murine listeriosis. J. Immunol. 153: 2093-2101. 
Rothe, J., Lesslauer, W., Lotscher, H., Lang, Y., Koebel, P., Kontgen, F., Althage A., Zinkernagel, R., Steinmetz, M., and Bluethmann, H. 1993. Mice lacking the tumour necrosis factor receptor 1 are resistant to TNF-mediated toxicity but highly susceptible to infection by Listeria monocytogenes. Nature. 364: 798-802.

Saldiva, P., Clarke, R. W., Coull, B. A., Stearns, R. C., Lawrence, J., Krishna, M. G. G., Diaz, E., Koutrakis, P., Suh, H., Tsuda, A., and Godleski, J. J. 2002. Lung inflammation induced by concentrated ambient air particles is related to particle composition. Am. J. Respir. Crit. Care Med. 165: 1610-1617.

Samet, J. M., Reed, W., Ghio, A. J., Devlin, R. B., Carter, J. D., Dailey, L. A., Bromberg, P. A., and Madden, M. C. 1996. Induciton of prostaglandin H synthase 2 in human airway epithelial cells exposed to residual oil fly ash. Toxicol Appl. Pharmcol. 141: 159168.

Samet, J. M., Stonehuerner, J., Reed, W., Devlin, R. B., Dailey, L. A., Kennedy, T. P., Bromberg, P. A., and Ghio, A. J. 1997. Disruption of protein tyrosine phosphate homeostasis in bronchial epithelial cells exposed to oil fly ash. Am. J. Phys. 272: L426L432.

Samet, J. M., Zeger, S. L., Dominici, F., Curriero, F., Coursac, I. Dockery, D. W., Schwartz, J., and Zanobetti, A. 2000a. The national morbidity, mortality, and air pollution study. Part II: Morbidity and mortality from air pollution in the United States. Res. Rep. Health Eff. Inst. 94:5-79.

Samet, J. M., Dominici, F., Curriero, F. C., Coursac, I., and Zeger, S. L. 2000b. Fine particulate air pollution and mortality in 20 U. S. cities, 1987-1994. N. Engl. J. Med. 343: 1742-1749.

Samet, J. M., Silbajoris, R., Huang, T., and Ilona, J. 2002. Transcription factor activation following exposure of an intact lung preparation to metallic particulate matter. Environ. Health Perspect. 110:985-990.

Schaumann, F., Borm, P. J. A., Herbrich, A., Knoch, J., Pitz, M., Schins, R. P. F., Luettig, B., Hohlfeld, J. M., Heinrich, J., and Krug, N. 2004. Metal-rich ambient particles (particulate matter 2.5 ) cause airway inflammation in healthy subjects. Am. J. Respir. Crit. Care Med. 170: 898-903.

Schluter, T., Berg, I., Dorger, M., and Gercken, G. 1995. Effect of heavy metal ions on the release of reactive oxygen intermediates by bovine alveolar macrophages. Toxicol. 98: 47-55.

Schroeder, W. H., Dobson, M., Kane, D. M., and Johnson, N. D. 1987. Toxic trace elements associated with airborne particulate matter: a review. J. Air Pollut. Control Assoc. 37: 1267-1285. 
Schwartz, J. 1994. What are people dying from on high pollution days? Environ. Res. 64:26-35.

Schwartz, J., Dockery, D. W., and Neas, L. M. 1996. Is daily mortality associated specifically with fine particles? J. Air Waste Manag. Assoc. 46: 927-939.

Schwartz, J., Ballester, F., Saez, M., Perez-Hoyos, S., Bellido, J., Cambra, K., Arribas, F., Canada, A., Perez-Boillos, M. J., and Sunyer, J. 2001. The concentration-response relation between air pollution and daily deaths. Environ. Health Perspect. 109: 10011006.

Seder, R. A., and Gazzinelli, R. T. 1999. Cytokines are critical in linking the innate and adaptive immune responses to bacterial, fungal, and parasitic infection. Adv. Intern. Med. 44: 353-88.

Sharma, R. P., Flora, S. J., Brown, D. B., and Oberg, S. G. 1987. Persistence of vanadium compounds in lungs after intratracheal instillation in rats. Toxicol. Indust. Health. 3: 321329.

Shen, X., Lee, K., and Konig, R. 2001. Effects of heavy metal ions on resting and antigen-activated CD4+ T cells. Toxicol. 169: 67-80.

Sherwood, R. L., Lippert, W. E., Goldstein, E., and Tarkington, B. 1981. Effect of ferrous sulfate aerosols and nitrogen dioxide on murine pulmonary defense. Arch. Environ. Health. 36: 130-135.

Shiloh, M. U., MacMicking, J. D., Nicholson, S., Brause, J. E., Potter, S., Marino, M., Fang, F., Dinauer, M., and Nathan, C. 1999. Phenotype of mice and macrophages deficient in both phagocyte oxidase and inducible nitric oxide synthase. Immunity. 10: 29-38.

Shrenk, H. H., Heimann, H., Clayton, G. D., Gafafer, W. M., and Wexler, H. 1949. Air pollution in Donora, PA: Epidemiology of the unusual smog episode in October, 1948, Preliminary Report. Public Health Bulletin No. 306. Washington, D. C.: U. S. Public Health Service.

Silbajoris, R., Ghio, A. J., Samet, J. M., Jaskot, R., Dreher, K. L., and Brighton, L. E. 2000. In vivo and in vitro correlation of pulmonary MAP kinase activation following metallic exposure. Inhal. Toxicol. 12: 453-468.

Simeonova, P. P., and Luster, M. I. 1995. Iron and reactive oxygen species in the asbestos-induced tumor necrosis factor-alpha response from alveolar macrophages. Am. J. Respir. Cell Mol. Biol. 12: 676-683.

Sjorberg, S-G. 1955. Vanadium bronchitis from cleaning oil-fired burners. Arch. Ind. Health. 11:505-512. 
Skachkova, M. A., Skachkov, M. V., Smolyagin, A. I., Boev, V. M., Vereshchagin, N. N., Mikhailova, I. V., Popova, E. V., and Skvortsov, V. O. 2001. Immune and interferon status in schoolchildren living in cities with different anthropologic load. Bull. Exp. Biol. Med. 4: 371-373.

Skornik, W. A., and Brain, J. D. 1983. Relative toxicity of inhaled metal sulfate salts for pulmonary macrophages. Am. Rev. Respir. Dis. 126: 297-303.

Smialowicz, R. J. 1998. Nickel. In: Immunotoxicology of environmental and Occupational Metals. Zelikoff, J. T., and Thomas, P. T., eds. Taylor and Francis, London, England. pp.163-194.

Smialowicz, R. J., Rogers, R. R., Riddle, M. M., and Stott, G. A. 1984. Immunologic effects of nickel. I. Suppression of cellular and humoral immunity. Environ. Res. 33: 413427.

Smialowicz, R. J., Rogers, R. R., Riddle, M. M., Gardner, R. J., Rowe, D. G., and Luebke, R. W. 1985. Immunologic effects of nickel. II. Suppression of natural killer (NK) cell activity. Environ. Res. 36: 56-66.

Smialowicz, R. J., Rogers, R. R., Riddle, M. M., Rowe, D. G., and Luebke, R. W. 1986. Immunologic studies in mice following in utero exposure to $\mathrm{NiCl}_{2}$. Toxicol. 38: 293-303.

Smialowicz, R. J., Rogers, R. R., Riddle, M. M., Luebke, R. W., Fogelson, L. D., nad Rowe, D. G. 1987. Effects of manganese, calcium, magnesium, and zinc on nickelinduced suppression of murine natural killer cell activity. J. Toxicol. Environ. Health. 20: $67-80$.

Smith, K. R., Veranth, J. M., Lighty, J. S., and Aust, A. E. 1998. Mobilization of iron from coal fly ash was dependent upon the particle size and the source of coal. Chem. Res. Toxicol. 11: 1494-1500.

Snow, E. T., and Costa, M. 1998. Nickel toxicity and carcinogenesis. In: Environmental and occupational medicine, $3^{\text {rd }}$ Ed. Rom, W. N. ed. Lippincott-Raven: Philadelphia, PA. Pp. 1057-1064.

Sorensen, M., Schinns, R. P., Hertel, O., and Loft, S. 2005. Transition metal in personnel samples of PM2.5 and oxidative stress in human volunteers. Cancer Epidem. Biomark. Prev. 14: 1340-1343.

Soukup, J. M., Ghio, A. J., and Becker, S. 2000. Soluble components of Utah Valley particulate pollution alter alveolar macrophage function in vivo and in vitro. Inhal. Toxicol. 12: 401-414. 
Spengler, J. D., and Thurston, G. 1983. Mass and elemental composition of fine and coarse particles in six U. S. cities. J. Air Pollut. Control Assoc. 33: 1162-1171.

Starzynski. Z., Marek, K., Kujawska, A., and Szymczak, W. 1996. Mortality among different occupational groups of workers with pneumoconiosis: results from a registerbased cohort study. Am. J. Ind. Med. 30: 718-725.

Steerenberg, P., Verlaan, A., De Klerk, A., Boere, A., Loveren, H., and Cassee, F. 2004. Sensitivity to ozone, diesel exhaust particles, and standardized ambient particulate matter in rats with Listeria monocytogenes-induced respiratory infection. Inhal. Toxicol. 16: 311-317.

Steinhoff, D., Mohr, U., and Hahnemann, S. 1991. Carcinogenesis studies with iron oxide. Exp. Pathol. 43: 189-194.

Stokinger, H. E. 1984. A review of world literature finds iron oxides noncarcinogenic. Am Ind. Hyg. Assoc. J. 45: 127-133.

Stoll, S., Jonuleit, H, Schmitt, E., Muller, G., Yarnauchi, H., Kurimoto, M., Knop, J., and Enk, A. H. 1998. Production of functional IL-18 by different subtypes of murine and human dendritic cells (DC): DC-derived IL-18 enhances IL-independent Th 1 development. Eur. J. Immunol. 28:3231-3239.

Sunderman, F. W. Jr., Hopfer, S. M., Lin, S. M., Plowman, M. C., Stojanivic, T., Wong, S. H., Zaharia, O., and Ziebka, L. 1989. Toxicity to alveolar macrophages in rats following parenteral injection of nickel chloride. Toxicol. Appl. Pharmacol. 100: 107118.

Takahashi, S., Yamada, M., Kondo, T., Sato, H., Furuya, K., and Tanaka, I. 1992. Cytotoxicity of nickel oxide particles in rat alveolar macrophages cultured in vitro. $J$. Toxicol. Sci. 17: 243-251.

Tao, F., Gonzalez-Flecha, B., and Kobzik, L. 2003. Reactive oxygen species in pulmonary inflammation by ambient particulates. Free. Radic. Biol. Med. 35: 327-340.

Toya, T., Fukuda, K., Takaya, M., and Arito, H. 2001. Lung lesions induced by intratracheal instillation of vanadium pentoxide powder in rats. Ind. Health 39: 8-15.

Tripp, C. S., Wolf, S. F. and Unanue, E. R. 1993. Interleukin 12 and tumor necrosis factor $\alpha$ are co-stimulators of interferon $\gamma$ production by natural killer cells in severe combined immunodeficiency mice with listeriosis, and interleukin 10 is a physiologic antagonist. Proc. Natl. Acad. Sci. USA. 90: 3725-3729.

Tripp, C. S. Gately, M. K., Hakimi, J., Ling, P., and Unanue, E. R. 1994. Neutralization of IL-12 decreases resistance to Listeria in SCID and C.B-17 mice. Reversal by IFNgamma. J. Immunol. 152: 1883-1887. 
Unanue, E. R. 1997. Studies in listeriosis show the strong symbiosis between the innate cellular system and the T cell response. Immun. Rev. 158: 11-25.

Vandenplas, O., Binard-van Cangh, F., Gregoire, J., Brumagne, A., and Larbanois, A. 2002. Fever and neutrophilic alveolitis caused by a vanadium-based catalyst. Occup.

Environ. Med. 59: 785-787.

Van der Veen, R. C., Dietlin, T. A., Pen, L., and Gray, J. D. 1999. Nitric oxide inhibits proliferation of T-helper 1 and 2 lymphocytes without reduction in cytokine secretion. Cell. Immunol. 193: 194-201.

Van der Veen, R. C., Dietlin, T. A., Gray, J. D., and Gilmore, W. 2000. Macrophagederived nitric oxide inhibits the proliferation of activated $\mathrm{T}$ helper cells and is induced during antigenic stimulation of resting T cells. Cell. Immunol. 199: 43-49.

Van Eeden, S. F., Tan, W. C., Suwa, T., Mukae, H., Terashima, T., Fujii, T., Qui, D., Vincent, R., and Hogg, J. C. 2001. Cytokines involved in the systemic inflammatory response induced by exposure to particulate matter air pollutants $\left(\mathrm{PM}_{10}\right)$. Am. J. Respir. Crit. Care Med. 164: 826-830.

Van Loveren, H., Rombout, P. J., Wagenaar, S. S., Walvoort, H. C., and Vos, J. G. 1988. Effects of ozone on the defense to a respiratory Listeria monocytogenes infection in the rat. Suppression of macrophage function and cellular immunity and aggravation of histopathology in lung and liver during infection. Toxicol. Appl. Pharmacol. 94: 374-393.

Van Zijverden, M., van der Pijl, A., Bol, M., van Pinxteren, F. A., de Haar, C., Penninks, A. H., van Loveren, H., and Pieters, R. Diesel exhaust, carbon black, and silica particles display distinct Th1/Th2 modulating activity. Toxicol. Appl. Pharmacol. 186: 131-139.

Veranth, J. M., Smith, K. R., Aust, A. E., Danise, S. L., Griffith, J. B., Hu, A. A., Huggins, M. L., and Lighty, J. S. 2000. Coal fly ash and mineral dust for toxicology and particle characterization studies: equipment and methods for PM2.5 - and PM1-enriched samples. Aerosol Sci. Technol. 32: 127-141.

Veronesi, B., Oortgiesen, M., Carter, J. D., and Devlin, R. B. 1999. Particulate matter initiates inflammatory cytokine release by activation of capsaicin and acid receptors in a human bronchial epithelial cell line. Toxicol. Appl. Phamacol. 154: 106-115.

Veronesi, B., de Haar, C., Roy, J., and Oortgiesen, M. 2002. Particulate matter inflammation and receptor sensitivity are target cell specific. Inhal. Toxicol. 14: 159-183.

Wang, L., Medan, D., Mercer, R., Overmill, D., Leonard, S., Castranova, V., Shi, X., Ding, M., Huang, C. and Rojanasakul, Y. 2003. Vanadium-induced apoptosis and pulmonary inflammation in mice: Role of reactive oxygen species. J. Cell. Physiol. 195: 99-107. 
Warheit, D. B., Hansen, J. F., Yuen, I. S., Kelly, D. P., Snajdr, S. I., and Hartsky, M. A. 1997. Inhalation of high concentrations of low toxicity dusts in rats results in impaired pulmonary clearance mechanisms and persistent inflammation. Toxicol. Appl.

Pharmacol. 145: 10-22.

Watkinson, W. P., Campen, M. J., and Costa, D. L. 1998. Cardiac arrhythmia induction after exposure to residual oil fly ash particles in a rodent model of pulmonary hypertension. Toxicol. Sci. 41: 209-216.

Watson, A. Y., and Brain, J. D. 1979. Uptake of iron oxide aerosols by mouse airway epithelium. Lab. Invest. 49: 450-459.

Watson, J. G., Rogers, C. F., and Chow, J. C. 1995. PM2.5 and PM10 variations in time and space. DRI Document No. 4204.1F. Report to U. S. Enivronmental Protection Agency.

Wesselius, L. J., Williams, W. L., Bailey, K., Vamos, S., O’Brien-Ladner, A. R., and Wiegmann, T. 1999. Iron uptake promotes hyperoxic injury to alveolar macrophages. Am. J. Respir. Crit. Care Med. 159: 100-106.

Wherry, J. G., Schreiber, R. D., and Unanue, E. R. 1991. Regulation of $\gamma$ interferon production by natural killer cells in SCID mice: Roles of tumor necrosis factor and bacterial stimuli. Infect. Immun. 59: 1709-1715.

White, L. R., Steinegger, A. F., and Schlatter, C. 1987. Pulmonary response following intratracheal instillation of potroom dust from an aluminum reduction plant into rat lung. Environ. Res. 42: 534-545.

Wichmann, H. E., Spix, C., Tuch, T. Wolke, G., Peters, A., Heinrich, J., Kreyling, W. G., and Heyder, J. 2000. Daily mortality and fine and ultra fine particles in Erfurt, Germany. Part I: Role of particle number and mass. Res. Rep. Health Eff. Inst. 98: 1-96.

Wilkins, E. T. 1954. Air pollution and the London fog of December, 1952. J. R. Sanitary Inst. 74: 1-21.

Williams, N. 1952. Vanadium poisoning from cleaning oil-fired burners. Br. J. Ind. Med. 9: 50-55.

WHO. 2003. Health aspects of air pollution with particulate matter, ozone, and nitrogen dioxide. Report on a WHO working group, Bonn, Germany, 13-15 January 2003. WHO regional office for Europe, Copenhagen.

Woodin, M. A., Liu, Y., Neuberg, D., Hauser, R., Smith, T., and Christiani, D. C. 2000. Acute respiratory symptoms in workers exposed to vanadium-rich fuel-oil ash. Am. J. Ind. Med. 37: 353-363. 
Wright, J. L., Harrison, N., Wiggs, B., and Churg, A. 1989. Quartz but not iron oxide causes air-flow obstruction, emphysema, and small airway lesions in the rat. Am. Rev. Respir. Dis. 138: 129-135.

Yamada, M., Takahashi, S., Sato, H., Kondo, T., Kikuchi, T., Furuya, K., and Tanaka, I. 1993. Solubility of nickel oxide particles in various solutions and rat alveolar macrophages. Biol. Trace Elem. Res. 36: 89-98.

Yang, H-M., Antonini, J. M., Barger, M., W., Butterworth, L., Roberts J. R., Ma, J. K., Castranova, V., and Ma, J. Y. 2001. Diesel exhaust particles suppress macrophage function and slow pulmonary clearance of Listeria monocytogenes in rats. Environ. Health Perspect. 109: 515-521.

Yin, X. J., Schafer, R., Ma, J. Y., Antonini, J. M., Weissman, D. N., Siegel, P. D., Barger, M. W., Roberts, J. R., and Ma, J. K. H. 2002. Alteration of pulmonary immunity to Listeria monocytogenes by diesel exhaust particles (DEPs). I. Effects of DEPs on early pulmonary responses. Environ. Health Perspect. 110: 1105-1111.

Yin, X. J., Schafer, R., Ma, J. Y., Antonini, J. M., Roberts, J. R., Weissman, D. N., Siegel, P. D., and Ma, J. K. 2003. Alteration of pulmonary immunity to Listeria monocytogenes by diesel exhaust particles (DEPs). II. Effects of DEPs on T-cellmediated immune responses in rats. Environ. Health Perspect. 111: 524-530.

Yin, X. J., Dong, C. C., Ma, J. Y., Antonini, J. M., Roberts, J. R., Stanley, C. F., Schafer, R., and Ma, J. K. 2004a. Suppression of cell-mediated immune responses to listeria infection by repeated exposure to diesel exhaust particles in brown Norway rats. Toxicol. Sci. 77: 263-271.

Yin, X. J., Ma, J. Y. C., Antonini, J. M., Castranova, V., and Ma, J. K. H. 2004b. Roles of reactive oxygen species and heme oxygenase-1 in modulation of alveolar macrophagemediated pulmonary immune responses to Listeria monocytogenes by diesel exhaust particles. Toxicol. Sci. 82: 143-153.

Yokota, S., Seki, T., Furuya, M., and Ohara, N. 2005. Acute functional enhancement of circulatory neutrophils after intratracheal instillation with diesel exhaust particles in rats. Inhal. Toxicol. 17:671-679.

Zelikoff, J. T., Schermerhorn, K. R., Fang, K., Cohen, M. D., and Schlesinger, R. B. 2002. A role for associated transition metals in the immunotoxicology of inhaled ambient particular matter. Environ. Health Perspect. 110 suppl 5: 871-875.

Zelikoff, J. T., Chen, L. C., Cohen, M. D., Fang, K., Gordon, T., Li, Y., Nadziejko, C., and Schlesinger, R. B. 2003. Effects of inhaled ambient particulate matter on pulmonary antimicrobial immune defense. Inhal. Toxicol. 15: 131-150. 
Zeromski, J., Jezewska, E., Sikora, J., and Kasprzak, K. S. 1995. The effects of nickel compounds on immunophentype and natural killer cell function of normal human lymphocytes. Toxicol. 97: 39-48.

Zhang, Q., and Huang, X. 2003. Induction of interleukin-6 by coal containing bioavailable iron is through both hydroxyl radical and ferryl species. J. Biosci. 28: 95100.

Zhou, Y-M., Zhong, C-Y., Kennedy, I. M., Leppert, V. J., and Pinkerton, K. E. 2003. Oxidative stress and NF- $\kappa \mathrm{B}$ activation in the lunsg of rats: a synergistic interaction between soot and iron particles. Toxicol. Appl. Pharmacol. 190: 157-169.

Zoller, W. H., Gordon, G. E., Gladney, E. S., et al. 1973. The source and distribution of vanadium in the atmosphere. In: Advances in chemistry series no. 123. Trace elements in the environment. Symosposium. Kothny, E. L., ed. Washington, D.C.: American Chemical Society, pp 31-47. 


\section{CURRICULUM VITAE \\ Jenny R. Roberts}

\section{PERSONAL INFORMATION}

National Institute for Occupational Safety and Health

Pathology and Physiology Research Branch

1095 Willowdale Rd.

Mailstop 2015

Morgantown, WV 26505

Phone: 304-285-5710 Fax: 304-285-5938

E-mail: jur6@cdc.gov

\section{EDUCATION}

High School Diploma

Bachelor Degrees

Ph.D.

\section{EXPERIENCE}

January, 1999 - Present

October, 1997 - December, 1998

October, 1997 - December, 1998
The Morgan School, Clinton, CT. June, 1991

Boston University, Boston, MA. BA in Biology with a Concentration in Marine Science, BA in Psychology. May 1996. Magna Cum Laude

West Virginia University, Morgantown, WV. West Virginia University Medical School. Department of Physiology and Pharmacology. May 2006.

National Institute of Occupational Safety and Health,Research Biologist

Harvard School of Public Health, Physiology Program, Biomedical Imaging Laboratory, Research Assistant II.

Harvard Medical School, Cell Biology
Department, Research Consultant

Harvard School of Public Health, Physiology Program, Biomedical Imaging Laboratory, Research Assistant I 


\section{AFFILIATIONS}

American Thoracic Society

American Physiological Society

Society of Toxicology

Allegheny - Erie Society of Toxicology

New England Society for Microscopy
August 2003 - Present

March 2003 - Present

September 2002 - Present

March, 1999 - Present

November, 1996 - 1999

\section{AWARDS}

Julie Betschart Award, Department

May, 2004

of Physiology and Pharmacology, WVU

Julie Betschart Award, Department

May, 2005

of Physiology and Pharmacology, WVU

\section{PUBLICATIONS (Chronologically)}

\section{Journal Articles}

Antonini, JM, Charron, TG, Lai, J, Roberts, JR, Blake, TL, and Rogers, RA. Application of laser scanning confocal microscopy in the analysis of particle-induced pulmonary fibrosis. Toxicol Sci 51: 126-134, 1999.

Antonini, JM, Starks, K, Roberts, JR, Millecchia, L, Yang, H-M, and Rao, KM. Changes in F-actin organization induced by hard metal particle exposure in rat pulmonary epithelial cells using laser scanning confocal microscopy. In Vitro Mol Toxicol 13: 5-16, 2000 .

Antonini, JM, Yang, H-M, Ma, JYC, Roberts, JR, Barger, MW, Butterworth, L, Charron, TG, and Castranova, V. Subchronic silica exposure enhances respiratory defense mechanisms and the pulmonary clearance of Listeria monocytogenes in rats. Inhal Toxicol 12: 1017-1036, 2000.

Antonini, JM, Roberts, JR, Yang, H-M, Barger, MW, Ramsey, D, Castranova, V, and Ma, JYC. Effect of short-term silica inhalation on the pulmonary clearance of a bacterial pathogen in Fischer 344 rats. Lung, 178(6): 341-350, 2000 Nov-Dec.

Antonini, JM, Roberts, JR, and Clarke, RW. Strain-related differences of non-specific respiratory defense mechanisms in rats using a pulmonary infectivity model. Inhal Toxicol, 13(1): 85-102, 2001 Jan.

Ding, M, Shi, X, Lu, Y, Huang, C, Leonard, S, Roberts, J, Antonini, J, Castranova, V, Vallyathan, V. Induction of activator protein-1 through reactive oxygen species by chrystalline silica in JB6 cells. J Biol Chem, 276(12): 9108-9114, 2001. 
Yang, H-M, Antonini, JM, Barger, MW, Butterworth, L, Roberts, JR, Ma, JKH, Castranova, V, and Ma, JYC. Diesel exhaust particles suppress macrophage function and slow the pulmonary clearance of Listeria monocytogenes in rats. Environ Health Perspect, 109(5): 515-521, 2001 May.

Joseph, P, Muchnok, TK, Klishis, ML, Roberts, JR, Antonini, JM, Whong, WZ, and Ong, T-M. Cadmium induced cell transformation and tumorigenesis are associated with transcriptional activation of c-fos, c-jun, and c-myc proto-oncogenes: role of cellular calcium and reactive oxygen species. Toxicol Sci, 61(2): 295-303, 2001 June.

Antonini, JM, Roberts, JR, Clarke, RW, Yang, H-W, Barger, MW, Jernigan, MR, Ma, JYC, and Weissman, DN. Effect of age on respiratory defense mechanisms and pulmonary bacterial clearance in Fischer 344 rats after intratracheal instillation of Listeria monocytogenes. Chest, 120: 240-249, 2001.

Castranova, V, Ma, JYC, Yang, H-M, Antonini, JM, Butterworth, L, Barger, MW, Roberts, JR, and Ma, JKH. Effect of exposure to diesel exhaust particles on the susceptibility of the lung to infection. Enivron Health Perspect, 109 Suppl 4: 609-612, August 2001.

Taylor, MD, Roberts, JR, Hubbs, AF, Reasor, MJ and Antonini, JM. Quantitative image analysis of drug-induced lung fibrosis using laser scanning confocal microscopy. Toxicol Sci, 67:295-302, 2002.

Yin, X-J, Schafer, R, Ma, JYC, Antonini, JM, Weissman, DN, Siegel, PD, Barger, MW, Roberts, JR, and Ma, JKH. Alteration of pulmonary immunity to Listeria monocytogenes by inhaled diesel exhaust particles (DEP) I. Effect of DEP on early pulmonary responses. Environ Health Perspect, 110:1105-1111, 2002.

Antonini, JM, Roberts, JR, Jernigan, MR, Yang, H-M, Ma, JYC, and Clarke, RW. Residual oil fly ash increases the susceptibility to infection and severely damages the lungs after pulmonary challenge with a bacterial pathogen. Toxicol Sci 70:110-119, 2002.

Gao, N, Jiang, B-H, Leonard, SS, Corum L, Zhang, Z, Roberts, JR, Antonini, JM, Zheng, JZ, Flynn, DC, Castranova, V, and Shi, X. p38 signaling-mediated hypoxiainducible factor 1 and vascular endothelial induction by $\mathrm{Cr}(\mathrm{VI})$ in DU145 human prostate carcinoma cells. J Biol Chem 277:45041-45048, 2002.

Lewis, AB, Taylor, MD, Roberts JR, Leonard, SS, Shi, X, and Antonini, JM. Role of Metal-induced Reactive Oxygen Species Generation in Lung Responses Caused by Residual Oil Fly Ash. J Biosciences, 28:13-18, 2003. 
Antonini, JM, Lewis, AB, Roberts, JR, and Whaley, DA. Pulmonary effects of welding fumes: review of worker and experimental animal studies. Am J Ind Med, 43:350-360, 2003.

Taylor, MD, Antonini, JM, Roberts, JR, Leonard, SS, Shi, X, Gannett, PM, Hubbs, AF, and Reasor, MJ. Intratracheal amiodarone administration to F344 rats directly damages lung airway and parenchymal cells. Toxicol Appl Pharmacol, 188:92-103, 2003.

Zeidler, PC, Chen, F, Butterworth, L, Andrew, ME, Roberts, JR, Robinson, VA, Porter, DW, and Castranova, V. Response of alveolar macrophages from inducible nitric oxide synthase knockout or wild type mice to an in vitro lipopolysaccharide or silica exposure. J Toxicol Environ Health, 66: 995-1013, 2003.

Yin, X-J, Schafer, R, Ma, JYC, Antonini, JM, Roberts, JR, Weissman, DN, Siegel, PD, and $\mathrm{Ma}, \mathrm{JKH}$. Alteration of pulmonary immunity to Listeria monocytogenes by diesel exhaust particles (DEPs) II. Effects of DEPs on T-cell-mediated immune responses in rats. Environ Health Perspect, 111:524-530, April 2003.

Tinkle, SS, Antonini, JM, Rich, BA, Roberts, JR, and Adkins, EJ. Particle penetration of the skin as a route of exposure in chronic beryllium disease. Environ Health Perspect, 111:1202-1208, July 2003.

Taylor, MD, Roberts, JR, Leonard, SS, Shi, X, and Antonini, JM. Effects of welding fumes of differing composition and solubility on free radical production and acute lung injury and inflammation in rats. Toxicol Sci, 75:181-191, 2003.

Antonini, JM, Roberts, JR, Taylor, MD, Yin, X-J, Stone, S, Moseley, Ma, JKH, Frazer, DG, Ma, JYC, and Castranova, V. Effect of asphalt inhalation exposure at simulated road paving conditions prior to bacterial infection on lung defense responses in rats. Inhal Toxicol, 15:1347-1368, 2003.

Antonini, JM, Taylor, MD, Leonard, SS, Lawryk, NJ, Shi, X, Clarke, RW, and Roberts, JR. Metal composition and solubility determine lung toxicity induced by residual oil fly ash collected from different sites within a power plant. Mol Cell Biochem, 255:257-265, 2004.

Leonard, SS, Roberts, JR, Antonini, JM, Castranova, V, and Shi, X. $\mathrm{PbCrO}_{4}$-mediated cellular responses via reactive oxygen species. Mol Cell Biochem, 255:171-179, 2004.

Antonini, JM, Taylor, MD, Zimmer, AT, and Roberts, JR. Pulmonary responses to welding fumes: Role of metal constituents. J Toxicol Environ Health, 67:233-249, 2004.

Roberts, JR, Taylor, MD, Castranova, V, Clarke, RW, and Antonini, JM. Soluble Metals associated with residual oil fly ash increase morbidity and lung injury after bacterial infection in rats. J Toxicol Environ Health, 67:251-263, 2004. 
Antonini, JM, Taylor, MD, Millecchia L, Bebout, AR, and Roberts, JR. Suppression in lung defense responses after bacterial infection in rats pretreated with different welding fumes. Toxicol Appl Pharmacol, 200:206-218, 2004.

Yin, X, Dong, C, Ma, JYC, Antonini, JM, Roberts, JR, Stanley, CF, Schafer, R, and Ma, JKH. Suppression of cell-mediated immune responses to Listeria infection by repeated exposure to diesel exhaust particles in Brown Norway rats. Toxicol Sci, 77:263-271, 2004.

Scabilloni, JF, Wang, L, Antonini, JM, Roberts, JR, Castranova, V, and Mercer, RR. Matrix metalloproteinase induction in fibrosis and fibrotic nodule formation due to silica inhalation. Am J Physiol- Lung Cell Mol Physiol, 288:709-717, 2005.

Brower, SL, Roberts, JR, Antonini, JM, and Miller, MR. Difficulty demonstrating estradiol-mediated Erk1/2 MAPK phosphorylation in MCF-7 cells J Steroid Biochem Mol Biol, 96: 375-285, 2005.

Antonini, JM, Leonard, SS, Roberts, JR, Solano-Lopez, C, Young, S-H, Shi, X, and Taylor, MD. Effect of stainless steel manual metal arc welding fume on free radical production, DNA damage, and apoptosis induction. Mol Cell Biochem, 279: 17-23, 2005.

Dong, CC, Yin, XJ, Ma, JYC, Millecchia, L, Barger, MW, Roberts, JR, Zhang, X-D, Antonini, JM, and Ma, JKH. Exposure of Brown Norway rats to diesel exhaust particles prior ro ovalbumin (OVA) sensitization elicits IgE adjuvant activity but attenuates OVAinduced airway inflammation. Toxicol Sci, 88: 202-212, 2005.

Dong, CC, Yin, XJ, Ma, JYC, Millecchia, L, Wu, Z-X, Barger, MW, Roberts, JR, Antonini, JM, Dey, RD, and Ma, JKH. Effect of diesel exhaust particles on allergic reactions and airway responsiveness in ovalbumin-sensitized Brown Norway rats. Toxicol Sci, 88: 150-160, 2005.

Yin, XJ, Dong, CC, Ma, JYC, Antonini, JM, Roberts, JR, Barger, MW, and Ma, JKH. Sustained effect of inhaled diesel exhaust particles on T lymphocyte-mediated immune responses against Listeria monocytogenes. Toxicol Sci, 88: 73-81, 2005.

Hu, X., Roberts, JR, Apopa, PL, Kan, YW, and Ma, Q. Accelerated ovarian failure induced by 4-vinyl cyclohexene diepoxide in Nrf2 null mice. Mol Cell Biol, 26: 940-954, 2006.

Wang, L, Scabilloni, JF, Antonini, JM, Castranova, V, Rojanasakul, Y, Roberts, JR, Zhang Z, and Mercer RR. Role of lung surfactant in phagocytic clearance of apoptotic cells by macrophages in rats. (Lab Invest, in press). 
Young, S-H, Roberts, JR, Antonini, JM. Pulmonary exposure to 1, 3- $\beta$-glucan alters adaptive immune responses in rats. (Inhal Toxicol, in press)

Solano-Lopez, C, Hubbs, AF, Zeidler-Erdely, PC, Reynolds, SH, Roberts, JR, Taylor, MD, Young, S-H, Castranova, V, and Antonini, JM. Welding fume exposure and associated inflammatory and hyperplastic changes in the lungs of tumor susceptible A/J mice. (Submitted to Toxicol Pathol, November 2005).

\section{Proceedings}

Antonini, JM, Starks, K, Millecchia, L, Roberts, J, and Roa, K. Changes in F-actin organization induced by hard metal particle exposure in rat pulmonary epithelial cells as observed by laser scanning confocal microscopy. Proceedings of Microcopy and Microanalysis 1999, Portland, OR, August 1999. Microscopy and Analysis 5(suppl 2): 492-493, August 1999.

Antonini, JM, Roberts, JR, Taylor, MD, and Clarke, RW. Effect of metal-containing environmental and occupational particulates on lung defense mechanisms. Proceedings of $9^{\text {th }}$ International Inhalation Symposium, Hannover, Germany, 2003. Effects of Air Contaminants on the Respiratory Tract - Interpretations from Molecules to Meta Analysis, Uwe Heinrich, ed., Fraunhofer IRB Verlag, Stuttgart, 2004, p283-299.

\footnotetext{
Abstracts

Sullivan, ER, Roberts, JR, Dowgiert, RK, Mervis, J, Lai, J, Antonini, JM, Mandel, R, Rees, D, Remold-O'Donnell, E, and Rogers, RA. Pulmonary distribution or recombinant human elastase inhibitor (rHEI) in rats. The New England Society for Electron Microscopy Annual Meeting, Woods Hole, MA, May, 1997.

Meadows, KN, Brismar, H, Mervis, J, Roberts, J, Lai, J, Brain, J, and Rogers, RA. Caveolae-mediated protein transport in cultured human bronchial epithelial cells (16HBE140-). Am J Resp Crit Med 157(3): A448, March, 1998.

Meadows, KN, Roberts, J, Ward, C, Lai, J, and Rogers, RA. Cholesterol sensitive caveolae-mediated protein transport in cultured human bronchial epithelial cells. The Fifth Joint Meeting of the Japan Society of Histochemistry and Cytochemistry and The Histochemical Society, San Diego, CA, July, 1998.

Antonini, JM, Jernigan, MR, Yang, H-M, Ma, JYC, Roberts, JR, Barger, MW, Butterworth, L, and Clarke, RW. Residual oil fly ash slows the clearance of Listeria monocytogenes from rat lungs. Society of Toxicology Meeting, Philadelphia, PA, March, 2000, Toxicol Sci: The Toxicologist, Vol 54: 316, 2000.
} 
Yang, H-M, Ma, JYC, Roberts, JR, Barger, MW, Butterworth, L, Castranova, V, and Antonini, JM. Exposure to silica activates macrophages and increases the pulmonary clearance of Listeria monocytogenes in rats. Society of Toxicology Meeting, Philadelphia, PA, March, 2000, Toxicol Sci: The Toxicologist, Vol 54: 318, 2000.

Tinkle, SS, Antonini, JM, Abrigo, BA, Adkins, EJ, and Roberts, JR. Particle penetration of the skin as a route of sensitization in occupational lung disease. Society of Toxicology Meeting, Philadelphia, PA, March, 2000, Toxicol Sci: The Toxicologist, Vol 54: 149, 2000.

Antonini, JM, Yang, H-M, Ma, JYC, Roberts, JR, Barger, MW, Jernigan, MR. Brain, JD, and Clarke, RW. Effect of age on acute mortality and pulmonary bacterial clearance in Fischer 344 rats following intratracheal instillation of Listeria monocytogenes. American Lung Association/American Thoracic Society International Conference, Toronto, Ontario, Canada, May 2000, Am J Respir Crit Care Med, Vol 161: A178, 2000.

Castranova, V, Ma, JYC, Antonini, JM, Yang, H-M, Butterworth, L, Barger, MW, Roberts, JR, Hahon, N, and Ma, JKH. Effect of exposure to diesel exhaust particles on the susceptibility of the lung to infection. Toxicology and Risk Assessment Approaches in the $21^{\text {st }}$ Century, Kings Island, OH, April 2000.

Ding, M, Shi, X, Lu, Y, Huang, C, Leonard, S, Roberts, JR, Antonini, JM, Castranova, $\mathrm{V}$, and Vallyathan, V. Involvement of reactive oxygen species in silica-induced AP-1 activation. Oxygen 2000: $7^{\text {th }}$ Annual Meeting of the Oxygen Society, San Diego, CA, November 2000.

Antonini, JM, Ebeling, AR, and Roberts, JR. Highly-soluble stainless steel welding fume slows the pulmonary clearance of a bacterial pathogen and severely damages the lungs after infection. Society of Toxicology Annual Meeting, San Francisco, CA, March, 2001. Toxicol Sci: The Toxicologist, Vol. 60: 424, 2001.

Taylor, MD, Antonini, JM, Roberts, JR, Van Dyke, K, Bowman, LL, Castranova, V, Hubbs, AF, Shi, X, Leonard, S, and Reasor, MJ. Intratracheal amiodarone administration to F344 rats causes acute damage to airway and parenchymal cells; the possible role of free radical generation. Society of Toxicology Annual Meeting, San Francisco, CA, March, 2001. Toxicol Sci: The Toxicologist, Vol. 60: 424, 2001.

Jospeh, P, Muchnok TK, Klishis, ML, Roberts, JR, Antonini, JM, Whong, W-Z, and Ong, T. Cadmium-induced cell transformation and tumorigenesis are associated with transcriptional activation of c-fos, c-jun, and c-myc proto-oncogenes: role of cellular calcium and reactive oxygen species. Society of Toxicology Annual Meeting, San Francisco, CA, March, 2001. Toxicol Sci: The Toxicologist, Vol. 60: 31, 2001.

Roberts, JR, Clarke, RW, and Antonini, JM. Soluble metals in residual oil fly ash suppress lung defense mechanisms and elevate acute mortality after infection. American 
Thoracic Society International Conference, San Fransisco, CA, May 2001, Am J Respir Crit Care Med 163: A495, 2001.

Taylor, MD, Roberts, JR, Hubbs, AF, Reasor, MJ, and Antonini, JM. Application of laser scanning confocal microscopy in the analysis of drug-induced lung fibrosis. American Thoracic Society International Conference, San Fransisco, CA, May 2001, Am J Respir Crit Care Med 163: A716, 2001.

Taylor, MD, Antonini, JM, Roberts, JR, Van Dyke, K, Bowmann, LL, Castranova, V, Hubbs, AF, Shi, X, Leonard, S, and Reasor, MJ. Intratracheal amiodarone administration to F344 rats causes acute lung damage: the possible role of free radical generation. $3^{\text {rd }}$ International Conference on Oxygen/Nitrogen Radicals: Cell Injury and Disease, Morgantown, WV, June 2002.

Lewis, AB, Roberts, JR, Taylor, MD, Leonard, SS, Shi, X, and Antonini, JM. Mechanisms of lung injury caused by residual oily fly ash: role of metal-induced reactive oxygen species generation. $3^{\text {rd }}$ International Conference on Oxygen/Nitrogen Radicals: Cell Injury and Disease, Morgantown, WV, June 2002.

Antonini, JM, Lewis, AB, Roberts, JR, Leonard, SS, Shi, X, and Taylor, MD. Generation of metal-induced reactive oxygen species by residual oil fly ash. Society of Toxicology Annual Meeting, Nashville, TN, March, 2002. Toxicol Sci: The Toxicologist, Vol. 66: 357, 2002.

Roberts, JR, Taylor, MD, Lewis, AB, and Antonini, JM. Removal of soluble metals from residual oil fly ash by Chelex protected rats from increases in mortality and lung injury after infection. Society of Toxicology Annual Meeting, Nashville, TN, March, 2002. Toxicol Sci: The Toxicologist, Vol. 66: 357, 2002.

Taylor, MD, Lewis, AB, Roberts, JR, Leonard, SS, Shi, X, and Antonini, JM. Effects of welding fumes on lung injury and inflammation: the possible role of free radical production. Society of Toxicology Annual Meeting, Nashville, TN, March, 2002. Toxicol Sci: The Toxicologist, Vol. 66: 193, 2002.

Antonini, JM, Taylor, MD, and Roberts, JR. Novel applications of confocal microscopy in lung toxicology. Experimental Biology Annual Meeting, New Orleans, LA, April 2002. FASEB J, Vol. 16: A738, 2002.

Yin, X-J, Schafer, R, Antonini, JM, Barger, MW, Dong, C-Z, Roberts, JR, Delarosa, P, $\mathrm{Ma}, \mathrm{JYC}$, and Ma, JKH. Alteration of innate and cell-mediated immunity to Listeria monocytogenes by short-term exposure to diesel exhaust particles. Experimental Biology Annual Meeting, New Orleans, LA, April 2002. FASEB J, Vol. 16: A962, 2002.

Tinkle, SS, Antonini, JM, Roberts, JR, Salmen, R, Depree, K, and Flint, MS. Cutaneous application of beryllium salts and oxide particles produces beryllium-specific 
sensitization in $\mathrm{C} 3 \mathrm{H} / \mathrm{HeOuJ}$ mice. Beryllium Research Symposium: Basic Mechanisms and Human Health, Bethesda, MD, June 2002.

Antonini, JM, Taylo,r MD, Lewis, AB, Yednock, JB, Leonard, SS, Lawryk, NJ, Shi, X, Clarke, RW, and Roberts, JR. Metal composition and solubility determine lung toxicity induced by residual oil fly ash collected from different sites within the same power plant. $2^{\text {nd }}$ Conference on Molecular Mechanisms of Metal Toxicity and Carcinogenesis, Morgantown, WV, September 2002.

Taylor, MD, Roberts, JR, Leonard, SS, Shi, X, and Antonini, JM. Effects of soluble and insoluble fractions of a manual metal arc stainless steel welding fume on free radical production and lung injury and inflammation. Society of Toxicology Annual Meeting, Salt Lake City, UT, March 2003. Toxicol Sci: The Toxicologist, Vol. 72:298, 2003.

Antonini, JM, Roberts, JR, and Taylor, MD. Exposure to stainless steel welding fume suppresses lung defense function after infection in rats. American Thoracic Society International Conference, Seattle, WA, May 2003, Am J Respir Crit Care Med, 167: A109, 2003.

Roberts, JR, Taylor, MD, and Antonini, JM. Soluble metals of residual oil fly ash compromise host defense in rats. American Thoracic Society International Conference, Seattle, WA, May 2003, Am J Respir Crit Care Med, A258, 2003.

Roberts, JR, Taylor, MD, Castranova, V, and Antonini, JM. Soluble metals associated with ROFA suppress lung immune defense and alter cytokine profiles after infection in rats. Society of Toxicology Annual Meeting, Baltimore, MD, March 2004. Toxicol Sci: The Toxicologist, Vol. 78:287, 2004.

Taylor, MD, Roberts, JR, Solano-Lopez, CE, Leonard, SS, Shi, X, and Antonini, JM. Effects of stainless steel manual metal arc welding fumes on DNA damage and apoptosis induction in vitro and in vivo. Society of Toxicology Annual Meeting, Baltimore, MD, March 2004. Toxicol Sci: The Toxicologist, Vol. 78:143, 2004.

Yin, X, Dong, C, Ma, JYC, Antonini, JM, Roberts, JR, and Ma, JKH. Repeated exposure to diesel exhaust particles causes suppression of cell-mediated immune responses to Listeria infection in Brown Norway rats. Society of Toxicology Annual Meeting, Baltimore, MD, March 2004. Toxicol Sci: The Toxicologist, Vol 78:99, 2004.

Solano-Lopez, C, Hubbs, A, Roberts, JR, Taylor, MD, Zeidler, P, Bowman L, Castranova, V, Reynolds, S, and Antonini, JM. Characterization of lung histopathology in $\mathrm{A} / \mathrm{J}$ mice after welding fume and silica treatment. American Thoracic Society International Conference, Orlando, FL, May 2004, Am J Respir Crit Care Med, A641, 2004.

Yin, X, Dong, C, Ma, JYC, Barger, MW, Roberts, JR, Antonini, JM, Siegel, PD, and $\mathrm{Ma}, \mathrm{JKH}$. Effect of inhaled diesel exhaust particles on allergic sensitization by ovalbumin 
in Brown Norway rats. American Thoracic Society International Conference, Orlando, FL, May 2004, Am J Respir Crit Care Med, A279, 2004.

Hu, X, Roberts, JR, Kan, YW, and Ma, Q. Essential role of Nrf2 in protection against ovarian follicle loss induced by 4-vinylcyclohexene and 4-vinylcyclohexene diepoxide in mice. ASBMB Annual Meeting and IUBMB Conference, Boston, MA, June 2004.

Antonini, JM, Taylor,MD, Solano-Lopez, C, Roberts, JR, Young, S-H, Leonard, SS, and Shi, X. Effect of welding fumes metal composition and solubility on free radical production and lung inflammation. $3^{\text {rd }}$ Conference on Molecular Mechanisms of Metal Toxicity and Carcinogenesis, Morgantown, WV, September 2004.

Roberts, JR, Young, S-H, Antonini, JM, and Castranova, V. Soluble nickel associated with residual oil fly ash increases susceptibility to pulmonary infection in rats. Society of Toxicology Annual Meeting, New Orleans, LA, March 2005. Toxicol Sci: The Toxicologist, Vol. 84:295-296, 2005.

Young, S-H, Roberts, JR, and Antonini, JM. Immune response to zymosan-induced pulmonary inflammation in rats. Society of Toxicology Annual Meeting, New Orleans, LA, March 2005. Toxicol Sci: The Toxicologist, Vol. 84: 192, 2005.

Yin, XJ, Dong, CC, Ma, JYC, Barger, MW, Roberts, JR, Antonini, JM, Siegel, PD, and $\mathrm{Ma}, \mathrm{JKH}$. How diesel exhaust particle exposure affects ovalbumin-induced airway sensitization and allergic response in rats. $10^{\text {th }}$ international Conference on Occupational Respiratory Diseases; Occupational Respiratory Hazards in the $21^{\text {st }}$ Century: Best Practices for Prevention and Control, Beijing, China, April 2005.

Roberts, JR, Mercer, RR, Young, S-H, Porter, D, Castranova, V, and Antonini, JM. Inflammation and fate of fluorescently-labeled quantum dots following pulmonary treatment of rats. $1^{\text {st }}$ International Symposium on Nanotoxicology: Biomedical Aspects, Miami, FL, January, 2006.

Antonini, JM, Roberts, JR, Stone, S, Chen, B, and Frazer, D. Suppression of Lung Defense Responses After Bacterial Infection in Rats Exposed by Inhalation to Stainless Steel Welding Particles. $1^{\text {st }}$ International Conference on Nanotoxicology: Biomedical Aspects, Miami, FL, January 2006.

Kisin, E, Murray, A, Roberts, JR, Antonini, JM, Reynolds, J, Kommineni, C, Kagan, V, and Shvedova, A. Lung defense responses after bacterial infection in mice pretreated with single wall carbon nanotubes. $1^{\text {st }}$ International Conference on Nanotoxicology: Biomedical Aspects, Miami, FL, January 2006.

Roberts, JR, and Antonini, JM. Soluble chromium in welding fume increases susceptibility to pulmonary bacterial infection in rats. Society of Toxicology Annual Meeting, San Diego, CA, March 2006. Toxicol Sci: The Toxicologist 90: 216, 2006. 
Young, S-H, Roberts, JR, and Antonini, JM. Pre-exposure to Zymosan Enhances Lung Defense Mechanisms and Accelerates the Pulmonary Clearance of a Bacterial Pathogen in Rats. Society of Toxicology Annual Meeting, San Diego, CA, March 2006. Toxicol Sci: The Toxicologist 90: 212, 2006.

Zeidler-Erdely, P, Young, S-H, Roberts, JR, Reynolds, SH, and Antonini, JM. Comparative inflammatory lung response in A/J and C57BL/6J mice exposed to stainless steel welding fume. Society of Toxicology Annual Meeting, San Diego, CA, March 2006. Toxicol Sci: The Toxicologist 90: 216, 2006.

Antonini, JM, Stone, S, Chen, B, Roberts,JR, Frazer, A, Donlin, M, Cumpston, J, and Frazer, D. Acute effects of stainless steel welding fume inhalation on lung injury, inflammation, and defense responses. Society of Toxicology Annual Meeting, San Diego, CA, March 2006. Toxicol Sci: The Toxicologist 90: 215-216, 2006. 